Supported cobalt nanocrystals as model catalysts for the Fischer-Tropsch synthesis

Tom van Deelen 
ISBN: 978-94-6375-821-5

Cover design: Jennifer Tas

Printed by: Ridderprint, The Netherlands 


\title{
Supported cobalt nanocrystals as model catalysts for the Fischer-Tropsch synthesis
}

\author{
Gedragen kobaltnanokristallen als \\ modelkatalysatoren voor de \\ Fischer-Tropschsynthese
}

(met een samenvatting in het Nederlands)

\author{
Proefschrift \\ ter verkrijging van de graad van doctor aan de \\ Universiteit Utrecht \\ op gezag van de \\ rector magnificus, prof.dr. H.R.B.M. Kummeling, \\ ingevolge het besluit van het college voor promoties \\ in het openbaar te verdedigen op
}

maandag 5 oktober 2020 des middags te 2.30 uur

door

\section{Tom Wijnand van Deelen}

\author{
geboren op 9 december 1991 \\ te Soest
}


Promotor: Prof. dr. ir. K.P. de Jong

Dit proefschrift werd (mede) mogelijk gemaakt met financiële steun van Shell Global Solutions en de Nederlandse Organisatie voor Wetenschappelijk Onderzoek (CHIPP 731.013.301) 


\section{Contents}

$\begin{array}{lll}\text { Chapter } 1 & \text { General introduction } & 7\end{array}$

Chapter 2 Assembly and activation of supported cobalt nanocrystal catalysts for the Fischer-Tropsch synthesis

Chapter 3 Cobalt nanocrystals on carbon nanotubes in the Fischer-Tropsch synthesis: the impact of support oxidation

Chapter 4 Preparation of cobalt nanocrystals supported on metal oxides to study particle growth in Fischer-Tropsch catalysts

Chapter 5 Disk-shaped Cobalt Nanocrystals as Fischer-Tropsch Synthesis

Catalysts under Industrially Relevant Conditions

Chapter 6 Control of metal-support interactions in heterogeneous catalysts to enhance activity and selectivity

Chapter 7 Activity enhancement of cobalt catalysts by tuning metal-support interactions

Chapter 8a Summary and concluding remarks

8b Nederlandse samenvatting

Appendices

List of publications and presentations

Acknowledgements

Curriculum vitae 


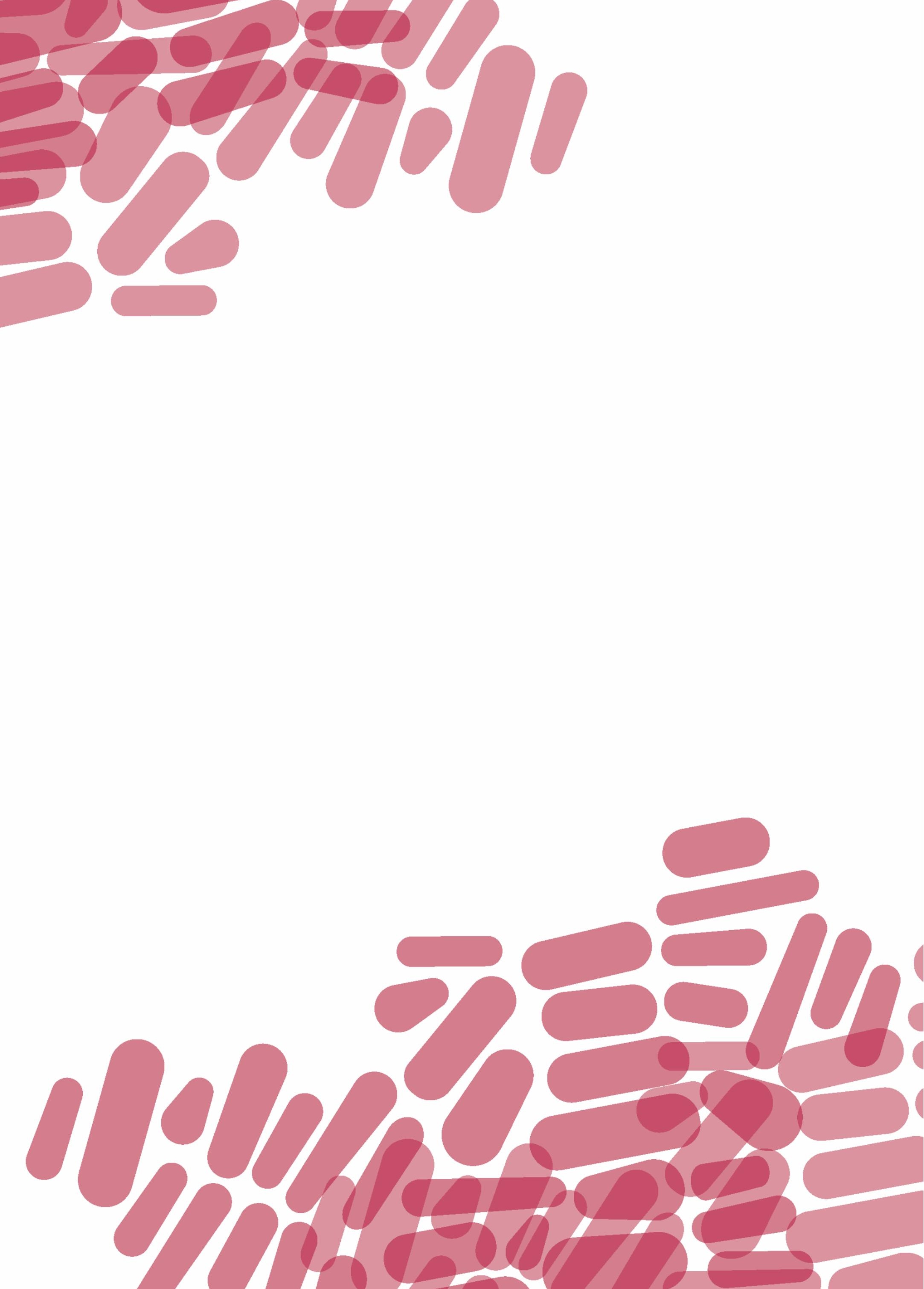




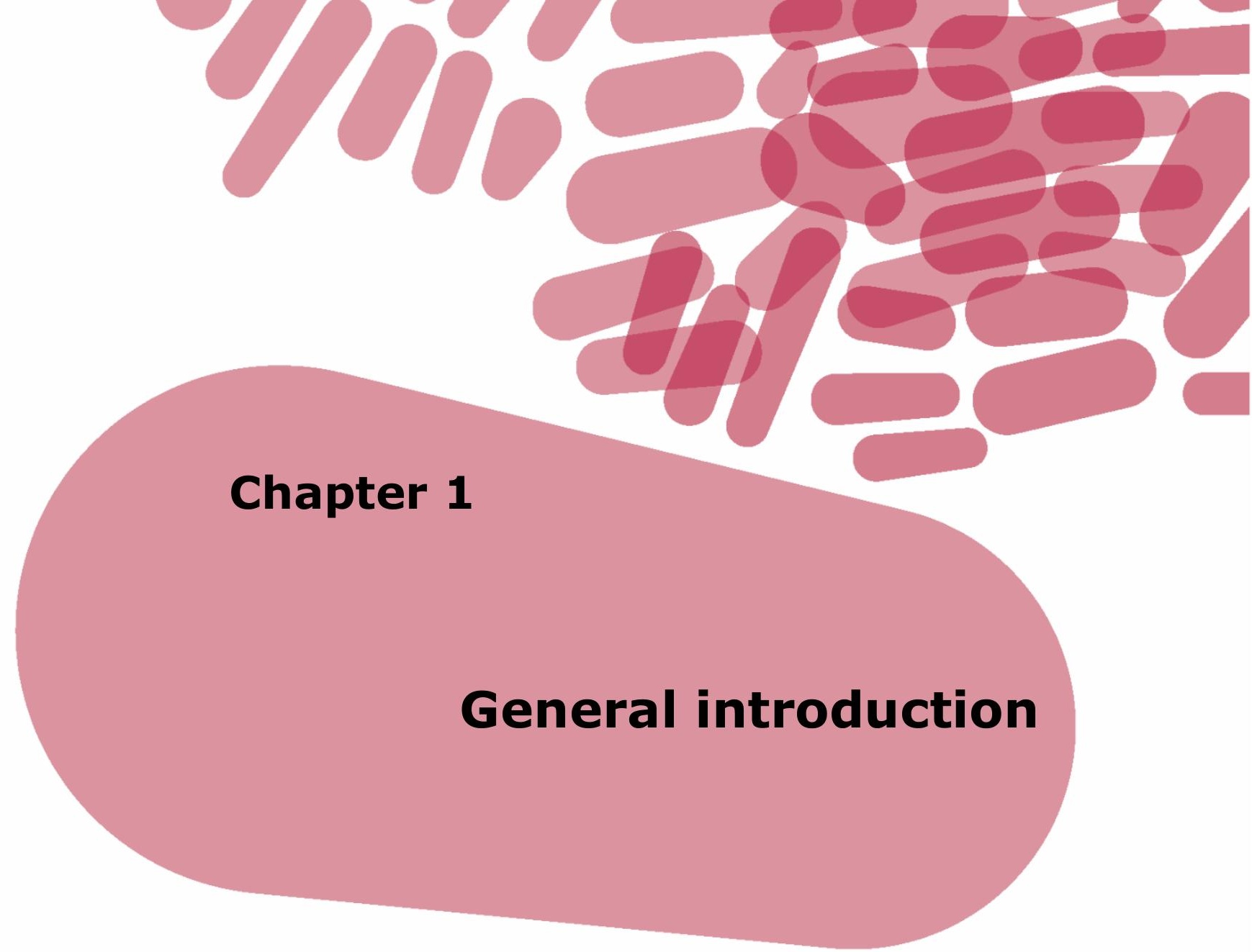




\subsection{Catalysis}

A catalyst is defined as 'A substance that increases the rate of a reaction without modifying the overall standard Gibbs energy change in the reaction'1 or, in other words, a catalyst speeds up a chemical reaction without changing the equilibrium of that reaction. In addition, a catalyst is in principle not consumed during the reaction. Catalysis is one of the key technologies in the chemical industry, since $95 \%$ (by volume) or $80 \%$ (by added value) of all chemical products are manufactured using catalysis. ${ }^{2}$ Industrial catalysis can be divided into two categories: homogeneous and heterogeneous catalysis. Catalysis is classified as homogenous when the catalyst is in the same phase as the reactants and conversely, a heterogenous catalyst is in a different phase than the reactants with the catalyst usually being a solid and the reactants being fluids (gasses and/or liquids). ${ }^{1}$ Homogenous catalysts are mainly responsible for the production of polymers and fine chemicals; heterogenous catalysts are involved in $80 \%$ of the catalytic processes, for example major petrochemical and refining processes as well as ammonia synthesis. ${ }^{2}$ Homogenous catalysts are reasonably well understood at the molecular level, while heterogenous catalysts are less defined and often feature a whole range of structures and catalytically active sites. One of the main reasons why heterogeneous catalysts have the widest applicability in industrial chemical processes is more facile handling and catalysts separation. ${ }^{3}$ Apart from enabling current chemical processes at high efficiency, catalysis is also envisaged to play a crucial role in addressing energy and climate challenges in the (near) future.

\subsection{Gas-to-liquids}

New fossil fuel reserves are still being discovered, especially given the current developments in shale gas and oil in the USA. When considering the restrictions on $\mathrm{CO}_{2}$ emissions as established in the 2015 Paris Climate Change Conference agreement, the use of fossil fuels might not be limited by supply but rather by emissions. Simultaneously, the global energy demand is expected to increase until 2040 mainly because of economic growth in developing countries (Figure 1.1a). ${ }^{4}$ To meet this increase in demand, energy consumption from all sources is expected to grow in absolute sense, including fossil fuels. Figure 1.1b shows the projection of the demand for liquid fuels broken down per type of end-user. ${ }^{4}$ The demand for liquid fuels will grow in the coming two decades mainly for transportation (cars, trucks and non-road), and particularly for heavy trucks and aviation, where alternative energy carriers are as yet absent. It seems thus that liquid fuels will be needed in the coming decades, making it important to produce and apply these as efficiently as possible in order to limit $\mathrm{CO}_{2}$ emissions.

Alternatives to traditional oil-based liquid fuels include liquified natural gas, biofuels and synthetic liquid fuels. The environmental benefit of synthetic liquid fuels strongly depends on the type of carbon-containing feedstock and overall process efficiency. ${ }^{5}$ For example, if associated gas from oil fields can be used as feedstock and flaring can be prevented, that offers a major reduction in overall carbon footprint. Synthetic liquid fuels can be obtained using so called X-to-liquids technology, with X being coal (CTL), natural gas (GTL) or biomass (BTL). The first step of XTL is to generate synthesis gas (an $\mathrm{H}_{2} / \mathrm{CO}$ mixture) from the feedstock. In a second step, the synthesis gas is converted in the Fischer- 
Tropsch synthesis (FT) into hydrocarbons, which are then refined in traditional installations to yield ultra-clean synthetic liquid fuels or chemicals. ${ }^{6}$ Currently, 0.5 million barrels per day $\left(\mathrm{Mb} \cdot \mathrm{d}^{-1}\right)$ of synthetic liquid fuels are produced using XTL, excluding China's rapidly growing CTL production ${ }^{7}$ In comparison, around $100 \mathrm{Mb} \cdot \mathrm{d}^{-1}$ of liquid fuel are produced from crude oil. ${ }^{8}$
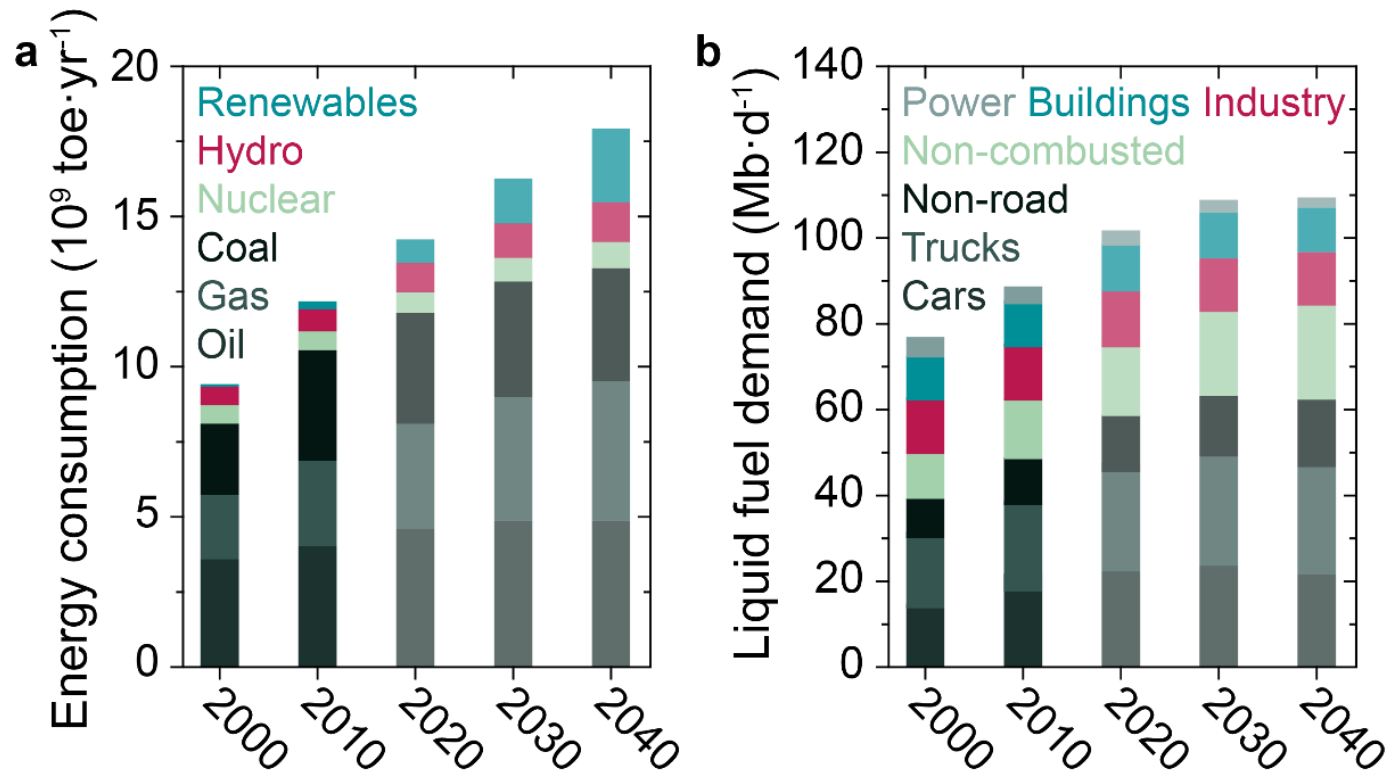

Figure 1.1. Global energy scenarios projected until 2040. a Primary energy consumption forecast in tonnes of oil equivalent per year (toe $\left.\cdot \mathrm{yr}^{-1}\right)$ and $\mathbf{b}$ breakdown of the projected demand for liquid fuels per consumer category in million barrels per day $\left(\mathrm{Mb} \cdot \mathrm{d}^{-1}\right)$. Adapted from ref. ${ }^{4}$

FT is a polymerization reaction of $\mathrm{CH}_{\mathrm{x}}$-monomers that takes place on a metal surface and produces predominantly linear alkanes (Equation 1.1) and $\alpha$-alkenes (Equation 1.2). ${ }^{9}$ The consequence of a polymerization reaction is that a statistical distribution of products of variable chain length is obtained following the Anderson-Schulz-Flory (ASF) distribution. The average chain length of the products is then determined by the chain growth probability ( $\alpha$, Figure 1.2). Industrial plants typically run at high $\alpha$ to avoid high selectivities towards undesirable methane and the long-chain wax products are subsequently cracked to maximize production in the fuel range. Apart from the main reaction, secondary reactions can take place, such as alkene readsorption or isomerization, which generally lead to deviations from the ASF distribution and to byproduct formation. Furthermore, water-gas shift (WGS, Equation 1.3) can occur as a major side reaction. WGS increases the $\mathrm{H}_{2} / \mathrm{CO}$ ratio inside the reactor by consuming part of the CO feed together with formed $\mathrm{H}_{2} \mathrm{O}$ in the reaction to produce $\mathrm{H}_{2}$ and $\mathrm{CO}_{2}$.

$$
\begin{aligned}
& n \mathrm{CO}+(2 n+1) \mathrm{H}_{2} \rightleftarrows \mathrm{C}_{n} \mathrm{H}_{2 n+2}+n \mathrm{H}_{2} \mathrm{O} \\
& n \mathrm{CO}+2 n \mathrm{H}_{2} \rightleftarrows \mathrm{C}_{n} \mathrm{H}_{2 n}+n \mathrm{H}_{2} \mathrm{O} \\
& \mathrm{CO}+\mathrm{H}_{2} \mathrm{O} \rightleftarrows \mathrm{CO}_{2}+\mathrm{H}_{2}
\end{aligned}
$$




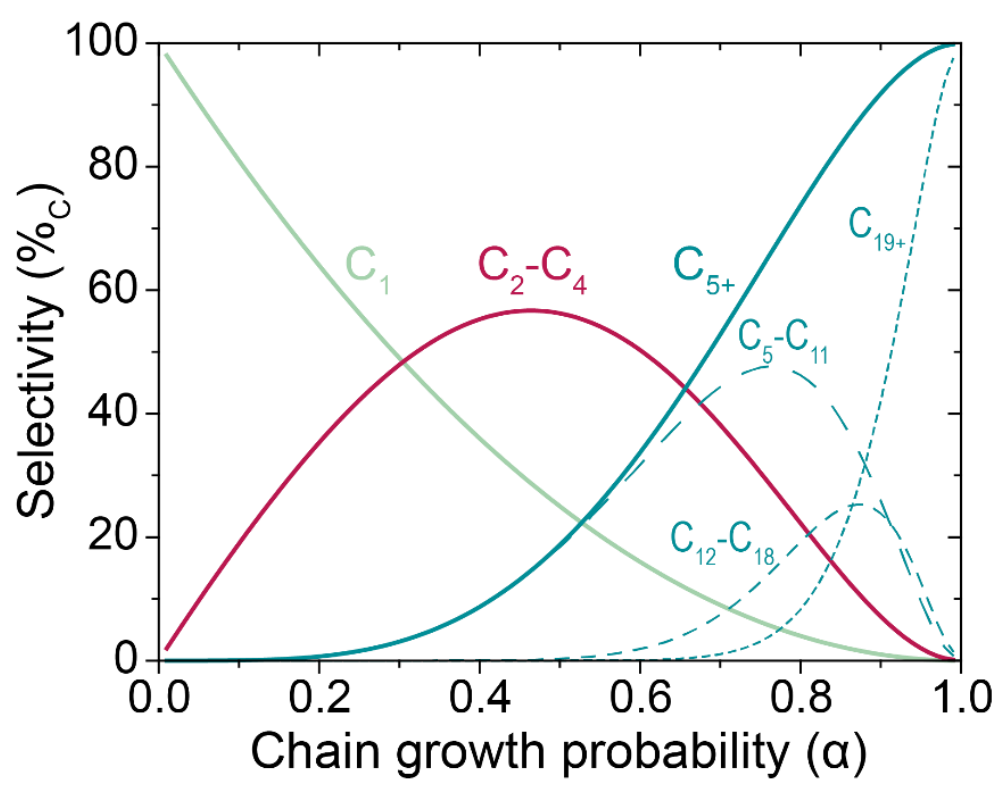

Figure 1.2. Product distribution as a function of chain growth probability $(\alpha)$.

\subsection{Fischer-Tropsch synthesis catalysts}

All group 8-10 metals are active in FT, but Fe, $\mathrm{Co}$, Ni and Ru have the highest activity. ${ }^{10}$ However, Ni has too high methane selectivity and forms nickel carbonyls under practical conditions and $\mathrm{Ru}$ is too expensive and scarce, so Fe and Co are mostly used in industry..11 Of the two, $\mathrm{Fe}$ is cheaper and more resistant to sulfur poisoning, but Co has higher productivity at high conversion and has negligible WGS activity at low temperatures. ${ }^{12}$ Whether Fe or Co is the most effective catalyst depends strongly on the available feedstock, because the $\mathrm{H}_{2}$ : $\mathrm{CO}$ ratio of the synthesis gas varies with feedstock and gasification process. Synthesis gas derived from coal typically has an $\mathrm{H}_{2}: \mathrm{CO}$ molar ratio of $0.5-1$ whereas synthesis gas from natural gas has a composition of $\mathrm{H}_{2}: \mathrm{CO} \sim 2 .{ }^{13} \mathrm{The}_{2}$ : $\mathrm{CO}$ stoichiometry of the FT reaction is approximately 2 (Equations 1.1 and 1.2), so GTL-synthesis gas can be used as is, while the composition of CTL-synthesis gas needs to be adjusted in situ by WGS, meaning that up to $50 \% \mathrm{CO}_{2}$-selectivity is obtained. This lowers the carbon efficiency of the process substantially. Fe has high WGS activity at typical operating temperatures and is therefore more suitable for CTL, while Co has no appreciable WGS activity and is thus preferred for GTL. Furthermore, Co is operated at low temperature $\left(200-300{ }^{\circ} \mathrm{C}\right)$, while $\mathrm{Fe}$ can be operated at low temperature as well as high temperature $\left(>300^{\circ} \mathrm{C}\right)$ to produce mainly short-chain alkenes..$^{14,15}$

Focusing on cobalt-based FT catalysts under GTL conditions $\left(\mathrm{H}_{2}: \mathrm{CO}=2\right)$, the current high price of Co motivates efficient usage of the metal. In metal-based catalysts, where reactions take place at the metal surface, that can be achieved by nanosizing to maximize the surface-to-bulk ratio. Because metal nanoparticles (NP) are thermodynamically unstable, these NP are dispersed over a support material. ${ }^{16}$ The support essentially acts as a spacer for the NP, thereby preventing agglomeration and sintering, and provides mechanical strength at the macroscale in a reactor. Typical supports for industrial Co catalysts are metal oxides $\left(\mathrm{TiO}_{2}, \mathrm{Al}_{2} \mathrm{O}_{3}\right.$ and $\left.\mathrm{SiO}_{2}\right)$, which are abundant and stable and can positively influence FT performance, especially in the case of $\mathrm{TiO}_{2}{ }^{7}, 1,18$ In addition, 
nanostructured carbon is employed in academic studies, because it has the advantages of inertness, i.e. no mixed metal-support compound formation, and facilitated imaging. ${ }^{19,20}$

The performance of cobalt catalysts in FT varies with the structure of the Co NP. For example, the intrinsic activity (activity per surface site or turnover frequency, TOF) is constant for large Co NP but the TOF and $\mathrm{C}_{5_{+}}$-selectivity drop sharply when the NP become smaller than 6-8 nm. ${ }^{21-23}$ More recently, the hep crystal structure of Co was found to be more active than fcc. ${ }^{24-28}$ Nevertheless, many questions still remain, such as the effect of Co NP shape and metal-support interactions as well as the mechanism of Co-catalyzed FT at the molecular scale. Linking the structure of Co NP to their catalytic performance is highly challenging and requires fundamental research on model catalysts in which specific parameters can be precisely controlled and decoupled from others, e.g. changing NP size without changing Co loading or support interactions.

\subsection{Model catalyst synthesis}

Conventional catalyst synthesis techniques, such as impregnation or precipitation, have in common that a metal precursor solution is applied to a support and metal (oxide) NP are then generated on the support using heat treatments. ${ }^{29}$ An alternative approach to produce model catalysts for fundamental studies is to synthesize metal particles separately using colloidal chemistry, which provides more opportunity to control their properties (size, shape or crystal structure), and anchor them to the support in a separate step. ${ }^{30}$

The synthesis of metallic colloids takes place in a solvent where a metal precursor decomposes in the presence of capping agents/ligands. These ligands are typically amphiphilic molecules such as fatty acids and linear amines, or polymers such as PVP. ${ }^{31}$ Decomposition of the metal precursor can for example occur under the influence of temperature or chemical agents. As the precursor decomposes, initial nuclei form that grow into colloidal nanocrystals (NC). ${ }^{32}$ The ligands stabilize the NC surface and isolate the growing NC from each other, preventing unbridled sintering and growth of the NC into a bulk phase. Through selective binding, these ligands also control much of the NC properties.$^{30}$ Colloidal synthesis of NC has already been extensively researched, although most work focused on noble metals and the number of synthesis methods to obtain cobalt $\mathrm{NC}$ in the relevant size regime for catalytic models studies $(1-50 \mathrm{~nm})$ is rather limited.

The pre-formed colloidal NC have to be uniformly distributed over a support to prevent sintering and deactivation. The nature of the interaction between $\mathrm{NC}$ and support in general is still uncertain. In the case of NC and carbon nanotubes (CNT), one explanation is that $\mathrm{NC}$ binding proceeds via electronic interactions with the $\Pi$-electrons of the graphene network, as was proposed for Pt-Co and Pt-Ni NC on CNT. ${ }^{33}$ Furthermore, the NC are competitively bound to the support and ligands, as indicated by NC detachment from the support in the presence of excess ligand. ${ }^{33,34}$ Alternatively, iron oxide NC were proposed to bind through van der Waals forces, explaining why the NC bound less strong to oxidized CNT compared to pristine CNT. ${ }^{35}$

Often, the ligands have to be removed prior to reaction to obtain an accessible and reactive metal surface and to be sure that the ligands do not negatively affect the reaction. ${ }^{36,37}$ Several options are available to remove ligands, including thermal, plasma and UV/ozone treatments. ${ }^{37-39}$ It is, however, questionable if and to what extent each of these 
treatments affect the initially well-defined NC. Alternatively, the ligands can be replaced for other, more desirable ones through ligand exchange, as was done for example to introduce sulfur and sodium promoters for an Fe-NC-based catalyst for the FischerTropsch to olefins process. ${ }^{40}$ Overall, both the attachment of NC to the support and their activation are relatively unexplored areas of research in particular for non-noble metals, even though they are essential for proper application of colloidal NC as supported model catalysts.

Despite the potential advantages, research involving supported NC catalysts is not straightforward; certain challenges are associated with NC-based catalysts that are quite unique in the field of catalyst synthesis. If colloids are to be successfully applied as supported (model) catalysts several practical constraints on the synthesis method emerge. We identify the following points as important considerations, depending on the intended application.

1) The synthesis method should give high control over the NC properties.

2) The synthesis method should be reproducible and insensitive to subtle variations.

3) The synthesis method should require no elements/compounds that could act as poison or promoter and lead to unrepresentative performance.

4) The interparticle interactions should be limited to facilitate uniform NC distribution over the support.

5) The ligands should not bind the NC too strongly to facilitate competitive NC binding to the support and enable complete ligand removal using convenient treatments.

6) The attachment and ligand removal procedure should not affect the NC properties.

7) The synthesis should be scalable so that reasonable amounts of catalysts are obtained. This is more challenging for industrial application than for lab-scale model studies.

8) For model studies, NC properties should be adjustable by variation of only few synthesis parameters while maintaining others as constant as possible.

\subsection{Cobalt nanocrystals as Fischer-Tropsch catalysts}

Co-NC can be utilized in FT either unsupported in the liquid phase ${ }^{41-43}$, or as supported catalysts in lab-scale FT experiments. ${ }^{23,44-54}$ In case of the supported catalysts, these NC were produced either via reverse-micelles or microemulsions ${ }^{23,44,47}$, solvothermal synthesis ${ }^{48,49}$, hot injection of $\mathrm{Co}_{2}(\mathrm{CO})_{8}^{51-54}$, or chemical reduction of a Co salt ${ }^{45,46,50}$. In all of these cases, the NC were attached to oxidic supports $\left(\mathrm{Al}_{2} \mathrm{O}_{3}, \mathrm{SiO}_{2}\right.$ or $\left.\mathrm{TiO}_{2}\right)$ through mixing of the colloidal suspension with the support or pore filling impregnation, all at ambient temperature. The ligands were removed via high-temperature thermal treatment, except for three cases ${ }^{45,46,54}$ where the as-prepared NC-based catalysts were directly reduced and low intrinsic activities were obtained. The NC-based catalysts were often applied to investigate Co particle size effects. However, besides NC size, colloidal techniques could also allow control over the crystal structure, shape and composition and therefore, more diverse model studies can in principle be performed to shed light on Co structureperformance relationships in FT. ${ }^{55}$ Simultaneously, NC-based catalyst synthesis as a whole, and in particular the application of base metal NC such as Co, should be investigated in more detail to realize this potential. 


\subsection{Scope of this thesis}

The goal of this research is to link the structure of supported cobalt catalysts to their performance in the Fischer-Tropsch synthesis using advanced model catalysts. Supported model systems are prepared through colloidal synthesis techniques and, in addition, this work aims to investigate and expand the applicability of nanocrystal-based catalysts for fundamental studies.

Chapter 2 describes how to attach the NC to a support and how to mildly activate them in order to obtain highly active Fischer-Tropsch (FT) catalysts.

The cause for the difference in FT performance between pristine and surface-oxidized carbon nanotubes (CNT) is investigated in Chapter 3. Well-defined Co-NC/CNT catalysts and advanced characterization techniques (STEM-EDX mapping, in situ XRD) are applied to isolate these effects.

The preparation of a range of $\mathrm{Co}-\mathrm{NC}$ of different sizes $(3,6,9$ and $12 \mathrm{~nm})$ on $\mathrm{TiO}_{2}$ and $\mathrm{SiO}_{2}$ supports is reported in Chapter 4. Using these catalysts, the effect of particle size and support on the growth of Co-NC during activation and FT is investigated.

Chapter 5 describes the synthesis of disk-shaped $\mathrm{Co}-\mathrm{NC}$ supported on $\mathrm{SiO}_{2}$. Their FT performance is compared to that of spherical $\mathrm{Co}-\mathrm{NC}$ and related to the structure of the catalysts.

Recent literature on the interaction between metal nanoparticles and supports is reviewed in Chapter 6 with a focus on strategies to tune metal-support interactions to enhance catalytic performance.

Chapter 7 focuses on cobalt-support interactions that occur specifically on reducible supports such as $\mathrm{TiO}_{2}$ and $\mathrm{Nb}_{2} \mathrm{O}_{5}$. A straightforward reduction-oxidation-reduction treatment is reported that doubles the accessible metallic surface area and cobalt-weightbased activity, while maintaining constant intrinsic activity.

Finally, conclusions and an outlook are provided.

\section{References}

1. IUPAC. Compendium of Chemical Terminology, Electronic version (2018). Available at: http://goldbook.iupac.org/C00876.html. (Accessed: 9th January 2019).

2. Hagen, J. Industrial Catalysis: A Practical Approach, 3rd Edition. (Wiley-VCH, 2015).

3. Lefferts, L., Hanefeld, U. \& Bitter, J. H. Introduction. in Catalysis: An Integrated Textbook for Students (eds. Hanefeld, U. \& Lefferts, L.) 1-14 (Wiley-VCH, 2018).

4. BP. BP Energy Outlook (2018). Available at: https://www.bp.com/content/dam/bp/ en/corporate/pdf/energy-economics/energy-outlook/bp-energy-outlook-2018.pdf. (Accessed: 19th September 2019).

5. Armstrong, R. C. et al. The frontiers of energy. Nat. Energy 1,15020 (2016).

6. Wood, D. A., Nwaoha, C. \& Towler, B. F. Gas-to-liquids (GTL): A review of an industry offering several routes for monetizing natural gas. J. Nat. Gas Sci. Eng. 9, 196-208 (2012).

7. van Steen, E., Claeys, M., Möller, K. P. \& Nabaho, D. Comparing a cobalt-based catalyst with iron-based catalysts for the Fischer-Tropsch XTL-process operating at high conversion. Appl. Catal. A Gen. 549, 51-59 (2018). 
8. U.S. Energy Information Administration. Short-Term Energy Outlook December (2018). Available at: https://www.eia.gov/outlooks/steo/archives/dec18.pdf. (Accessed: 19th September 2019).

9. Iglesia, E. Design, synthesis, and use of cobalt-based Fischer-Tropsch synthesis catalysts. Appl. Catal. A Gen. 161, 59-78 (1997).

10. Vannice, M. A. The catalytic synthesis of hydrocarbons from $\mathrm{H}_{2} / \mathrm{CO}$ mixtures over the group VIII metals. J. Catal. 50, 228-236 (1977).

11. Schulz, H. Short history and present trends of Fischer-Tropsch synthesis. Appl. Catal. A Gen. 186, 3-12 (1999).

12. Khodakov, A. Y., Chu, W. \& Fongarland, P. Advances in the Development of Novel Cobalt Fischer-Tropsch Catalysts for Synthesis of Long-Chain Hydrocarbons and Clean Fuels. Chem. Rev. 107, 1692-744 (2007).

13. van der Laan, G. P. Kinetics, Selectivity and Scale Up of the Fischer-Tropsch Synthesis. PhD Thesis (University of Groningen, 1999). doi:10.1081/CR-100101170

14. Dry, M. E. The Fischer-Tropsch process: 1950-2000. Catal. Today 71, 227-241 (2002).

15. Torres Galvis, H. M. et al. Supported Iron Nanoparticles as Catalysts for Sustainable Production of Lower Olefins. Science 335, 835-838 (2012).

16. Lefferts, L., Hensen, E. J. M. \& Niemantsverdriet, J. W. Heterogeneous Catalysis. in Catalysis: An Integrated Textbook for Students (eds. Hanefeld, U. \& Lefferts, L.) 15-71 (Wiley-VCH, 2018).

17. Prieto, G. et al. Cobalt-Catalyzed Fischer-Tropsch Synthesis: Chemical Nature of the Oxide Support as a Performance Descriptor. ACS Catal. 5, 3323-3335 (2015).

18. Morales, F., de Smit, E., de Groot, F. M. F., Visser, T. \& Weckhuysen, B. M. Effects of manganese oxide promoter on the $\mathrm{CO}$ and $\mathrm{H}_{2}$ adsorption properties of titania-supported cobalt FischerTropsch catalysts. J. Catal. 246, 91-99 (2007).

19. Serp, P., Corrias, M. \& Kalck, P. Carbon nanotubes and nanofibers in catalysis. Appl. Catal. A Gen. 253, 337-358 (2003).

20. Bezemer, G. L. et al. Preparation of Fischer-Tropsch cobalt catalysts supported on carbon nanofibers and silica using homogeneous deposition-precipitation. J. Catal. 237, 291-302 (2006).

21. Bezemer, G. L. et al. Cobalt Particle Size Effects in the Fischer-Tropsch Reaction Studied with Carbon Nanofiber Supported Catalysts. J. Am. Chem. Soc. 128, 3956-3964 (2006).

22. den Breejen, J. P. et al. On the Origin of the Cobalt Particle Size Effects in Fischer-Tropsch Catalysis. J. Am. Chem. Soc. 131, 7197-7203 (2009).

23. Prieto, G., Martínez, A., Concepción, P. \& Moreno-Tost, R. Cobalt particle size effects in Fischer-Tropsch synthesis: structural and in situ spectroscopic characterisation on reverse micelle-synthesised Co/ITQ-2 model catalysts. J. Catal. 266, 129-144 (2009).

24. Ducreux, O., Rebours, B., Lynch, J., Roy-Auberger, M. \& Bazin, D. Microstructure of Supported Cobalt Fischer-Tropsch Catalysts. Oil Gas Sci. Technol. - Rev. d'IFP Energies Nouv. 64, 49-62 (2009).

25. Sadeqzadeh, M. et al. Identification of the active species in the working alumina-supported cobalt catalyst under various conditions of Fischer-Tropsch synthesis. Catal. Today 164, 62-67 (2011). 
26. Gnanamani, M. K., Jacobs, G., Shafer, W. D. \& Davis, B. H. Fischer-Tropsch synthesis: Activity of metallic phases of cobalt supported on silica. Catal. Today 215, 13-17 (2013).

27. Liu, J.-X., Su, H.-Y., Sun, D.-P., Zhang, B.-Y. \& Li, W.-X. Crystallographic Dependence of CO Activation on Cobalt Catalysts: HCP versus FCC. J. Am. Chem. Soc. 135, 16284-16287 (2013).

28. Lyu, S. et al. Role of Active Phase in Fischer-Tropsch Synthesis: Experimental Evidence of CO Activation over Single-Phase Cobalt Catalysts. ACS Catal. 8, 7787-7798 (2018).

29. Munnik, P., de Jongh, P. E. \& de Jong, K. P. Recent Developments in the Synthesis of Supported Catalysts. Chem. Rev. 115, 6687-6718 (2015).

30. Zaera, F. Nanostructured materials for applications in heterogeneous catalysis. Chem. Soc. Rev. 42, 2746-2762 (2013).

31. Jia, C.-J. \& Schüth, F. Colloidal metal nanoparticles as a component of designed catalyst. Phys. Chem. Chem. Phys. 13, 2457-2487 (2011).

32. An, K. \& Somorjai, G. A. Size and Shape Control of Metal Nanoparticles for Reaction Selectivity in Catalysis. ChemCatChem 4, 1512-1524 (2012).

33. Ritz, B. et al. Reversible Attachment of Platinum Alloy Nanoparticles to Nonfunctionalized Carbon Nanotubes. ACS Nano 4, 2438-2444 (2010).

34. Casavola, M., Hermannsdörfer, J., de Jonge, N., Dugulan, A. I. \& de Jong, K. P. Fabrication of Fischer-Tropsch Catalysts by Deposition of Iron Nanocrystals on Carbon Nanotubes. Adv. Funct. Mater. 25, 5309-5319 (2015).

35. Krans, N. A. et al. Attachment of Iron Oxide Nanoparticles to Carbon Nanotubes and the Consequences for Catalysis. ChemCatChem 10, 3388-3391 (2018).

36. Park, J. Y., Aliaga, C., Renzas, J. R., Lee, H. \& Somorjai, G. A. The Role of Organic Capping Layers of Platinum Nanoparticles in Catalytic Activity of CO Oxidation. Catal. Letters 129, 1-6 (2009).

37. Li, D. et al. Surfactant Removal for Colloidal Nanoparticles from Solution Synthesis: The Effect on Catalytic Performance. ACS Catal. 2, 1358-1362 (2012).

38. Roldan Cuenya, B. \& Behafarid, F. Nanocatalysis: Size- and shape-dependent chemisorption and catalytic reactivity. Surf. Sci. Rep. 70, 135-187 (2015).

39. Cargnello, M. et al. Efficient Removal of Organic Ligands from Supported Nanocrystals by Fast Thermal Annealing Enables Catalytic Studies on Well-Defined Active Phases. J. Am. Chem. Soc. 137, 6906-6911 (2015).

40. Casavola, M. et al. Promoted Iron Nanocrystals Obtained via Ligand Exchange as Active and Selective Catalysts for Synthesis Gas Conversion. ACS Catal. 7, 5121-5128 (2017).

41. Gual, A., Godard, C., Castillón, S., Curulla-Ferré, D. \& Claver, C. Colloidal Ru, Co and Fenanoparticles. Synthesis and application as nanocatalysts in the Fischer-Tropsch process. Catal. Today 183, 154-171 (2012).

42. Wang, H. et al. Platinum-Modulated Cobalt Nanocatalysts for Low-Temperature AqueousPhase Fischer-Tropsch Synthesis. J. Am. Chem. Soc. 135, 4149-4158 (2013).

43. Delgado, J. A., Claver, C., Castillón, S., Curulla-Ferré, D. \& Godard, C. Correlation between Hydrocarbon Product Distribution and Solvent Composition in the Fischer-Tropsch Synthesis Catalyzed by Colloidal Cobalt Nanoparticles. ACS Catal. 5, 4568-4578 (2015).

44. Fischer, N., van Steen, E. \& Claeys, M. Structure sensitivity of the Fischer-Tropsch activity and selectivity on alumina supported cobalt catalysts. J. Catal. 299, 67-80 (2013). 
45. Delgado, J. A. et al. Fischer-Tropsch synthesis catalysed by $\mathrm{small}^{\mathrm{TiO}_{2}}$ supported cobalt nanoparticles prepared by sodium borohydride reduction. Appl. Catal. A Gen. 513, 39-46 (2016).

46. Delgado, J. A. et al. Effect of polymeric stabilizers on Fischer-Tropsch synthesis catalyzed by cobalt nanoparticles supported on $\mathrm{TiO}_{2}$. J. Mol. Catal. A Chem. 417, 43-52 (2016).

47. Lögdberg, S. et al. Effect of water on the space-time yield of different supported cobalt catalysts during Fischer-Tropsch synthesis. Appl. Catal. A Gen. 393, 109-121 (2011).

48. Park, J.-Y. et al. Fischer-Tropsch catalysts deposited with size-controlled $\mathrm{Co}_{3} \mathrm{O}_{4}$ nanocrystals: Effect of Co particle size on catalytic activity and stability. Appl. Catal. A Gen. 411-412, 15-23 (2012).

49. Lee, Y.-J., Park, J.-Y., Jun, K.-W., Wook Bae, J. \& Prasad, P. S. S. Controlled Nanocrystal Deposition for Higher Degree of Reduction in $\mathrm{Co} / \mathrm{Al}_{2} \mathrm{O}_{3}$ Catalyst. Catal. Letters 130, 198-203 (2009).

50. Werner, S., Johnson, G. R. \& Bell, A. T. Synthesis and Characterization of Supported CobaltManganese Nanoparticles as Model Catalysts for Fischer-Tropsch. ChemCatChem 6, 2881-2888 (2014).

51. Melaet, G., Lindeman, A. E. \& Somorjai, G. A. Cobalt Particle Size Effects in the Fischer-Tropsch Synthesis and in the Hydrogenation of $\mathrm{CO}_{2}$ Studied with Nanoparticle Model Catalysts on Silica. Top. Catal. 57, 500-507 (2014).

52. Melaet, G. et al. Evidence of Highly Active Cobalt Oxide Catalyst for the Fischer-Tropsch Synthesis and $\mathrm{CO}_{2}$ Hydrogenation. J. Am. Chem. Soc. 136, 2260-2263 (2014).

53. Ralston, W. T., Melaet, G., Saephan, T. \& Somorjai, G. A. Evidence of Structure Sensitivity in the Fischer-Tropsch Reaction on Model Cobalt Nanoparticles by Time-Resolved Chemical Transient Kinetics. Angew. Chemie Int. Ed. 56, 7415-7419 (2017).

54. Herranz, T., Deng, X., Cabot, A., Guo, J. \& Salmeron, M. Influence of the Cobalt Particle Size in the CO Hydrogenation Reaction Studied by In Situ X-Ray Absorption Spectroscopy. J. Phys. Chem. B113, 10721-10727 (2009).

55. Beaumont, S. K. Recent developments in the application of nanomaterials to understanding molecular level processes in cobalt catalysed Fischer-Tropsch synthesis. Phys. Chem. Chem. Phys. 16, 5034-5043 (2014). 


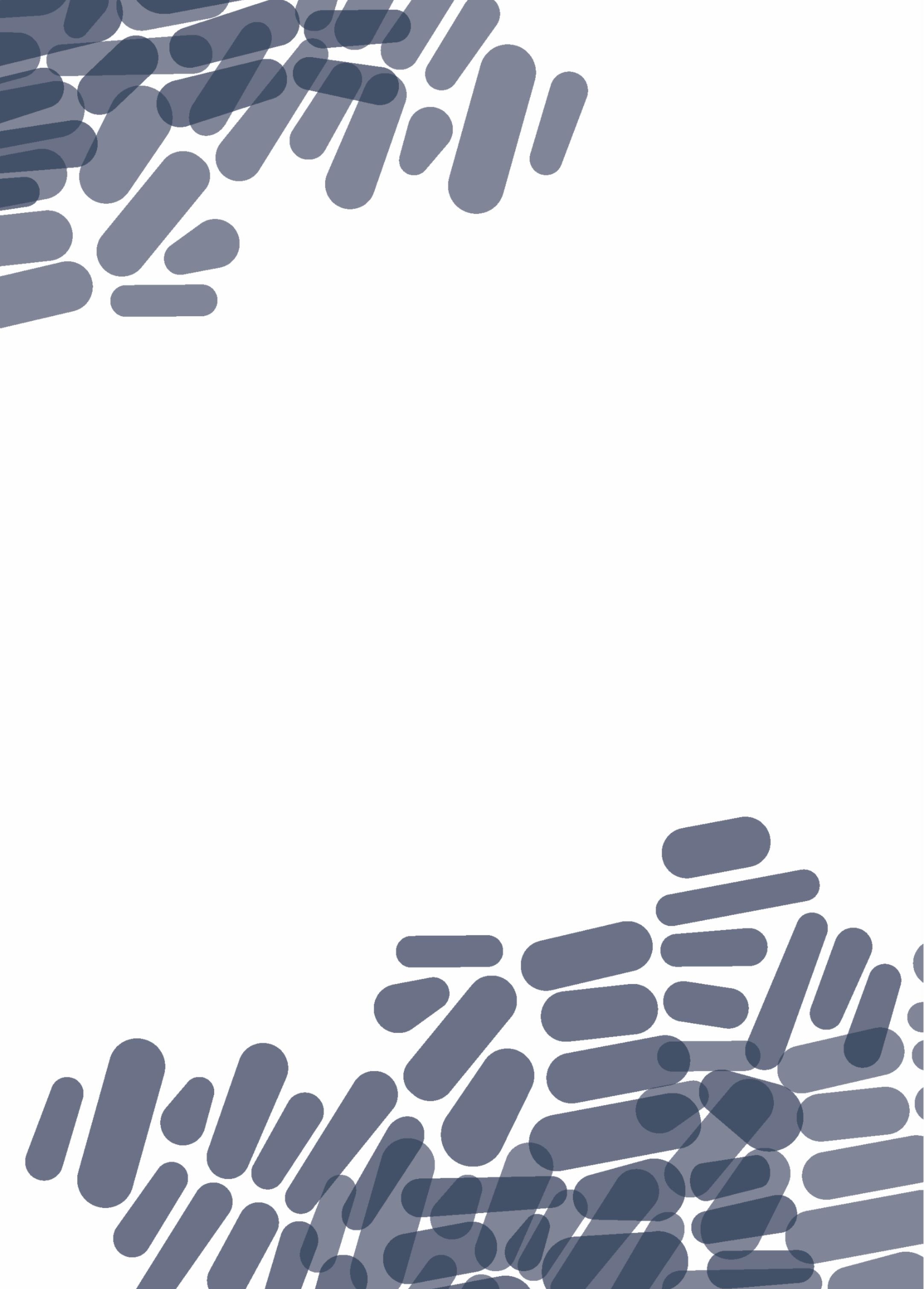




\subsection{Introduction}

Colloidal nanocrystals (NC) can be applied to synthesize well-defined supported model catalysts. ${ }^{1,2}$ However, two specific challenges arise in catalyst preparation when using colloids. Firstly, the NC have to be uniformly attached to a support. This is often achieved by mixing a NC suspension with the support followed by separation and washing. Such methods lead to uniform NC distributions only if the particle-support and particle-particle interactions are optimal. ${ }^{3-5}$ Secondly, the surfaces of NC are usually covered with stabilizing ligands, which limit accessibility of the metal surface for reactants and thereby hamper catalytic activity ${ }^{6,7}$ Ligands are commonly removed through oxidative treatments at high temperatures, but such severe conditions can affect for example the NC size and shape. ${ }^{8}$

These challenges have been investigated and largely resolved for noble-metal NC. ${ }^{9}$ This even led to the introduction of the first commercial NC-based catalysts containing Pd- or Pt-NC. ${ }^{10}$ In contrast, base-metal NC have been studied less for catalysis and are more challenging than noble-metal NC, because base metal NC are more susceptible to oxidation and magnetic interactions. ${ }^{11}$ Furthermore, common ligands, such as oleic acid, are hard Lewis bases and bind stronger to base metals, which are hard Lewis acids compared to noble metals. ${ }^{12}$

As a showcase of base metal NC, this study focusses on cobalt catalysts for the FischerTropsch (FT) synthesis. In FT, fuels and chemicals are produced from synthesis gas, a $\mathrm{H}_{2} / \mathrm{CO}$ mixture that can be derived from natural gas, coal or biomass. ${ }^{13}$ The understanding of FT could benefit from colloidal techniques to provide more precise structureperformance relationships that are not readily obtained otherwise. ${ }^{14,15}$ Supported cobalt catalysts for FT have been prepared using colloidal techniques. ${ }^{4,16-24}$ Most were activated through high-temperature (HT) oxidation followed by reduction to arrive at high intrinsic activities. ${ }^{4,16-21}$ When HT-oxidative treatments were omitted, significantly lower intrinsic activities were obtained. ${ }^{22-24}$ The development of a milder activation procedure would reduce the impact on the $\mathrm{Co}-\mathrm{NC}$ and thereby increase the potential of $\mathrm{Co}-\mathrm{NC}$ for future structure-performance studies. ${ }^{2,25}$ In this chapter, we investigated the effect of oxidative treatments on the attachment and activation of $\mathrm{Co}-\mathrm{NC}$ on carbon nanotubes (CNT) as FT catalysts.

\subsection{Experimental}

\subsubsection{Synthesis of $\mathrm{Co}(0)$ nanocrystals}

The synthesis of $\mathrm{Co}-\mathrm{NC}$ was based on the method reported by Iablokov et al. ${ }^{26}$ In this method, the introduction of elements that might ultimately affect the catalytic performance, such as phosphorus or sodium, was avoided. The synthesis was performed under $\mathrm{N}_{2}$ atmosphere on a Schlenk line. Typically, $65 \mathrm{mg}$ of oleic acid (Sigma-Aldrich, $90 \%$, technical grade) was transferred to a $100 \mathrm{~mL}$ 3-necked flask equipped with a cooler and two septa and evacuated at $120{ }^{\circ} \mathrm{C}$ for $30 \mathrm{~min}$. After flushing several times with $\mathrm{N}_{2}$, $7.5 \mathrm{~mL}$ of 1,2-dichlorobenzene (Sigma-Aldrich, $99 \%$, anhydrous) was inertly transferred to the flask and the mixture was heated to $174{ }^{\circ} \mathrm{C}$ under $750 \mathrm{rpm}$ magnetic stirring. Meanwhile, $270 \mathrm{mg}$ dicobalt octacarbonyl (Acros Organics, $95 \%$, stabilized) was dissolved 
in $1.5 \mathrm{~mL} 1,2-$ dichlorobenze in a glass vial inside a glovebox. Then, the cobalt precursor was rapidly injected into the flask containing the mixture at $174{ }^{\circ} \mathrm{C}$ and $750 \mathrm{rpm}$ magnetic stirring. The reaction was continued for 20 minutes and quenched afterwards using a water bath. Optionally, for low-temperature (LT) oxidized nanocrystals (NC), the Co-NC were exposed to air at room temperature under $650 \mathrm{rpm}$ magnetic stirring for $1 \mathrm{~h}$ by removing one septum in order to oxidize the NC to CoO. Subsequently, the colloidal suspension of $\mathrm{CoO}-\mathrm{NC}$ was divided over two glass centrifuge tubes, both were filled up to $20 \mathrm{~mL}$ with 2propanol (Sigma-Aldrich, >99\%, LC-MS Chromasolv) and centrifuged at $2200 \mathrm{G}$ for $20 \mathrm{~min}$. The supernatant was decanted and the precipitated colloids were redispersed in $0.5 \mathrm{~mL}$-hexane (Acros Organics, 99+\%) per tube using sonication. Again, the tubes were filled to $20 \mathrm{~mL}$ with 2-propanol, then centrifuged at $2200 \mathrm{G}$ for $20 \mathrm{~min}$ and the supernatant was decanted. This washing cycle was repeated 2 times more and finally the NC were redispersed in $2 \mathrm{~mL}$ toluene (Acros Organics, 99+\%, extra pure) and stored in a glass vial.

\subsubsection{Attachment of $\mathbf{C o}(0)$ nanocrystals to carbon nanotubes}

The support, CNT (Baytubes c150 HP), was first sieved to a fraction of 75-150 $\mu \mathrm{m}$. Co- and $\mathrm{CoO}-\mathrm{NC}(10 \mathrm{wt} \% \mathrm{Co})$ were emplaced on CNT by wet impregnation at elevated temperature under nitrogen atmosphere on a Schlenk line. ${ }^{3}$ To this end, $500 \mathrm{mg}$ CNT was transferred to a $100 \mathrm{~mL} 3$-necked flask equipped with a cooler, septum and glass plug. $5 \mathrm{~mL}$ 1-octadecene (Sigma-Aldrich, 90\%, technical grade) was mixed with the colloidal suspension (in toluene) and added to the CNT under $400 \mathrm{rpm}$ magnetic stirring. The suspension was degassed at $100{ }^{\circ} \mathrm{C}$ for 30 min during which the toluene evaporated, flushed several times with nitrogen and heated to $200{ }^{\circ} \mathrm{C}$ for $30 \mathrm{~min}$. After cooling down to room temperature, $\sim 20 \mathrm{~mL}$ acetone was added to the suspension and the supernatant was decanted. The precipitated catalyst was washed six times by first adding $2 \mathrm{~mL} n$-hexane followed by $6 \mathrm{~mL}$ acetone and decanting of the supernatant. Finally, the catalyst was dried at $60^{\circ} \mathrm{C}$ for $1 \mathrm{~h}$ in static air, at $120^{\circ} \mathrm{C}$ for $3 \mathrm{~h}$ in static air and at $80^{\circ} \mathrm{C}$ for $3 \mathrm{~h}$ under vacuum. Afterwards, the catalyst was again sieved to a fraction of $75-150 \mu \mathrm{m}$.

\subsubsection{High-temperature oxidative treatment of CoO-NC/CNT}

A batch of LT-oxidized CoO-NC on CNT was treated at high temperature in an oxidative environment. First, $100 \mathrm{mg}$ of catalyst was loaded in a U-shaped quartz reactor and $37.5 \mathrm{~mL} \cdot \mathrm{min}^{-1}$ of $20 \mathrm{vol} \% \mathrm{O}_{2}$ in $\mathrm{N}_{2}$ was led through the catalyst in fixed bed mode. The catalyst was heated to $250{ }^{\circ} \mathrm{C}$ for $2 \mathrm{~h}$ with $2{ }^{\circ} \mathrm{C} \cdot \mathrm{min}^{-1}$ and was afterwards cooled again to room temperature.

\subsubsection{Characterization}

A cryogenic transmission electron microscopy (cryo-TEM) sample of the suspension of metallic Co-NC under inert atmosphere was prepared using a vitrification robot (FEI Vitrobot Mark III). To prepare the sample, $3 \mu \mathrm{L}$ of sample was applied on a 200 mesh, lacey carbon-coated $\mathrm{Cu}$ grid inside an environmental chamber at $20{ }^{\circ} \mathrm{C}$ with $99 \%$ relative humidity of toluene. The grid was blotted with filter paper for $3 \mathrm{~s}$ and then vitrified by rapidly plunging the grid into liquid nitrogen $\left(-196^{\circ} \mathrm{C}\right)$. The total vitrification procedure took less than $10 \mathrm{~s}$ in order to minimize the contact time with air and to avoid oxidation of 
the Co-NC. Cryo-TEM was performed on a TU/e CryoTitan (FEI) operating at $300 \mathrm{kV}$ using low electron dose rates of $37 \mathrm{e} \cdot \AA^{-2} \cdot \mathrm{s}^{-1}$ at the magnification used. The crystal structure of the NC was analyzed using low dose selective area electron diffraction (LDSAED).

Transmission electron microscopy (TEM) was performed using a Tecnai 12 (FEI) operating at $120 \mathrm{kV}$. A sample of a CoO-NC suspension was prepared by dropcasting the colloidal suspension in hexane on a 200 mesh, carbon-coated $\mathrm{Cu}$ grid. In the case of CNT supported catalysts, the powder was first suspended in 2-propanol by sonication and subsequently dropcasted on a 200 mesh holey carbon-coated $\mathrm{Cu}$ grid. The particle sizes were manually determined using iTEM software and at least 200 particles were analyzed to obtain a statistical analysis. The reported diameters of all $\mathrm{Co}_{\mathrm{x}} \mathrm{O}_{\mathrm{y}}$ particles were converted to their equivalent metallic cobalt diameter and were volume/surface averaged $(\mathrm{D}[3,2])$. Reduced and passivated samples were corrected for a $3 \mathrm{~nm}$ CoO layer.

High-resolution transmission electron microscopy (HR-TEM) was carried out on a Talos F200X (FEI), equipped with a high-brightness field emission gun and operated at $200 \mathrm{kV}$. Sample preparation was identical to that of TEM.

$\mathrm{X}$-ray diffraction (XRD) was performed using a Bruker D2 Phaser using Co radiation $\left(\mathrm{K}_{\alpha}, \lambda=1.789 \AA\right)$. The diffractograms were analyzed and fitted using Bruker's Eva and Topas software. The reported crystallite size was calculated using the Scherrer equation after fitting the full scan from $20-100^{\circ} 2 \theta$ and analysis of the $\varepsilon-\mathrm{Co}$ [221], $\mathrm{CoO}$ [111] and $\mathrm{Co}_{3} \mathrm{O}_{4}$ [311] peaks.

Inductively coupled plasma-optical emission spectroscopy (ICP-OES) was measured on a SPECTRO ARCOS and samples were prepared by cobalt extraction in aqua regia.

Thermogravimetric analysis-mass spectrometry (TGA-MS) was carried out on a PerkinElmer Pyris 1 TGA. Approximately $5 \mathrm{mg}$ sample was pre-dried at $50{ }^{\circ} \mathrm{C}$ under $\mathrm{N}_{2}$ and subsequently analyzed while heating to $800{ }^{\circ} \mathrm{C}$ with $10{ }^{\circ} \mathrm{C} \cdot \mathrm{min}^{-1}$ under a flow of $25 \mathrm{vol} \% \mathrm{H}_{2}$ in $\mathrm{N}_{2}$.

\subsubsection{Fischer-Tropsch synthesis}

The performance of the catalysts was evaluated using an Avantium Flowrence 16 parallel reactor setup. Stainless steel, plug-flow reactors were first loaded with a mixture of 15$50 \mathrm{mg}$ of catalyst and $100 \mathrm{mg} \mathrm{SiC} \mathrm{diluent.} \mathrm{Then,} \mathrm{the} \mathrm{catalysts} \mathrm{were} \mathrm{reduced} \mathrm{in} \mathrm{situ} \mathrm{at} 350{ }^{\circ} \mathrm{C}$ for $8 \mathrm{~h}\left(1{ }^{\circ} \mathrm{C} \cdot \mathrm{min}^{-1}\right)$ in $25 \mathrm{vol} \% \mathrm{H}_{2}$ in He at 1 bar. Subsequently, the reactors were cooled to $180{ }^{\circ} \mathrm{C}$ and the pressure was increased to 20 bar under $\mathrm{H}_{2}$ flow. Finally, synthesis gas with $\mathrm{H}_{2} / \mathrm{CO}=2 \mathrm{v} / \mathrm{v}, 5 \mathrm{vol}^{\%}$ He as internal standard and GHSV $=2350-7850 \mathrm{~h}^{-1}$ was introduced and the reactors were heated to $220^{\circ} \mathrm{C}$ with $1{ }^{\circ} \mathrm{C} \cdot \mathrm{min}^{-1}$. The reaction products were analyzed online with an Agilent 7890A GC. The hydrocarbon products were separated on an Agilent J\&W PoraBOND Q column and analyzed by a FID. The permanent gasses were separated on a ShinCarbon ST column and quantified using a TCD.

The activity was reported as CO conversion, defined as $\mathrm{X}_{\mathrm{CO}}=\left(\mathrm{mol}_{\mathrm{CO}, \text { in }}-\mathrm{mol}_{\mathrm{CO}, \text { out }}\right) \cdot \mathrm{mol}_{\mathrm{CO}}$, in $^{-1}$, and as cobalt time yield and turnover frequency. For the TOF calculation, the surface averaged cobalt diameter of the spent catalysts from TEM was used to determine the metallic surface area, assuming spherical particles. From the metallic surface area, the number of surface cobalt atoms was derived using a cobalt cross sectional area of $0.0662 \mathrm{~nm}^{2}$ per atom. ${ }^{27}$ Furthermore, the selectivity towards $C_{1}$ to $C_{4}$ hydrocarbons was 
calculated as $\mathrm{S}_{\mathrm{Cn}}=\mathrm{mol}_{\mathrm{Cn}} \cdot \mathrm{n} \cdot\left(\mathrm{mol}_{\mathrm{CO}, \text { in }}-\mathrm{mol}_{\mathrm{CO}, \text { out }}\right)^{-1}$, where $\mathrm{n}$ is the carbon number $(1-4)$ of the product. The selectivity to $\mathrm{C}_{5+}$ was defined as $\mathrm{S}_{\mathrm{C} 5+}=1-\mathrm{S}_{\mathrm{C} 1-4}$.

\subsection{Results and discussion}

Co-NC were synthesized using colloidal techniques. Figure 2.1 provides an overview of the different treatments applied to the Co-NC, which will be discussed step by step in the following sections. The Co-NC were imaged after synthesis using cryogenic transmission electron microscopy (cryo-TEM). The Co-NC had neither been washed nor exposed to air. The as-synthesized $\mathrm{Co}-\mathrm{NC}$ were cubical and formed chains, indicating magnetic interactions between metallic Co-NC (Figure 2.2a). Electron diffraction experiments using cryo-TEM showed that the crystal structure of the freshly synthesized NC was $\varepsilon$-cobalt (Figure 2.2b, Appendix A, Table A1), which is a crystal structure of Co that is accessible via wet-chemical synthesis of Co-NC. ${ }^{28}$

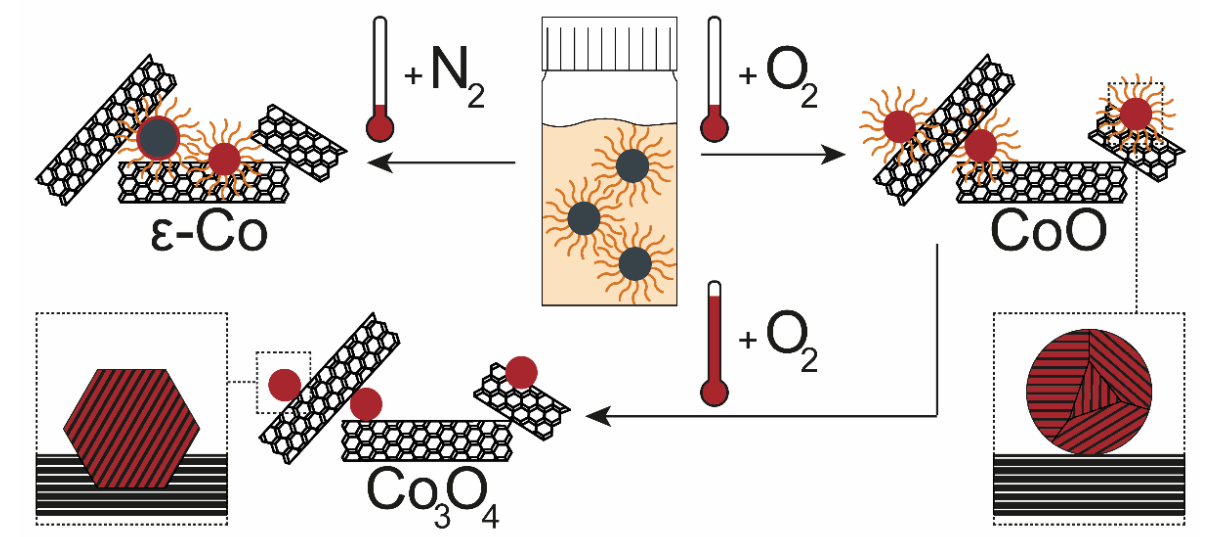

Figure 2.1. Schematic overview of the oxidative treatments applied to the NC. The treatments include no oxidation of $\mathrm{Co}-\mathrm{NC}$, low-temperature oxidation of $\mathrm{Co}-\mathrm{NC}$ to $\mathrm{CoO}-\mathrm{NC}$ and hightemperature oxidation of $\mathrm{CoO}-\mathrm{NC} / \mathrm{CNT}$ to $\mathrm{Co}_{3} \mathrm{O}_{4}-\mathrm{NC} / \mathrm{CNT}$.

Low-temperature (LT) oxidation of as-synthesized Co-NC was pursued by airexposure at ambient conditions during subsequent washing steps. This reproducibly resulted in particles of 6-7 nm with a narrow particle size distribution (Figure 2.2c,d) according to transmission electron microscopy (TEM). In a typical NC synthesis, a cobalt yield of $\sim 50 \%$ was obtained. The final NC after the washing steps consisted of $\mathrm{CoO}$, as inferred from electron diffraction (Appendix A, Figure A1, Table A1), and had become irregularly spherical, owing to their polycrystalline nature. No remaining metallic cores were detected for the $\mathrm{COO}-\mathrm{NC}$ using either TEM or electron diffraction. Consequently, the magnetic interactions were reduced and the formation of chains on the TEM grid was no longer observed (Figure 2.2c).

Both $\mathrm{Co}-$ and $\mathrm{CoO}-\mathrm{NC}$ were attached to untreated, hydrophobic CNT through mixing at elevated temperature. This method resulted in nearly quantitative attachment of the NC to the CNT with some variation of the final metal loading due to a slightly variable $\mathrm{NC}$ concentration in the suspension. The supported $\mathrm{Co}-\mathrm{NC}$ and $\mathrm{CoO}-\mathrm{NC}$ were designated 
$\mathrm{Co} 1 / \mathrm{CNT}$ and $\mathrm{Co} 2 / \mathrm{CNT}$, respectively. In the case of Co1/CNT, oxidation through airexposure was avoided by omitting the washing steps after the Co-NC synthesis. The CoNC were exposed to air for the first time during drying after attachment to the CNT.

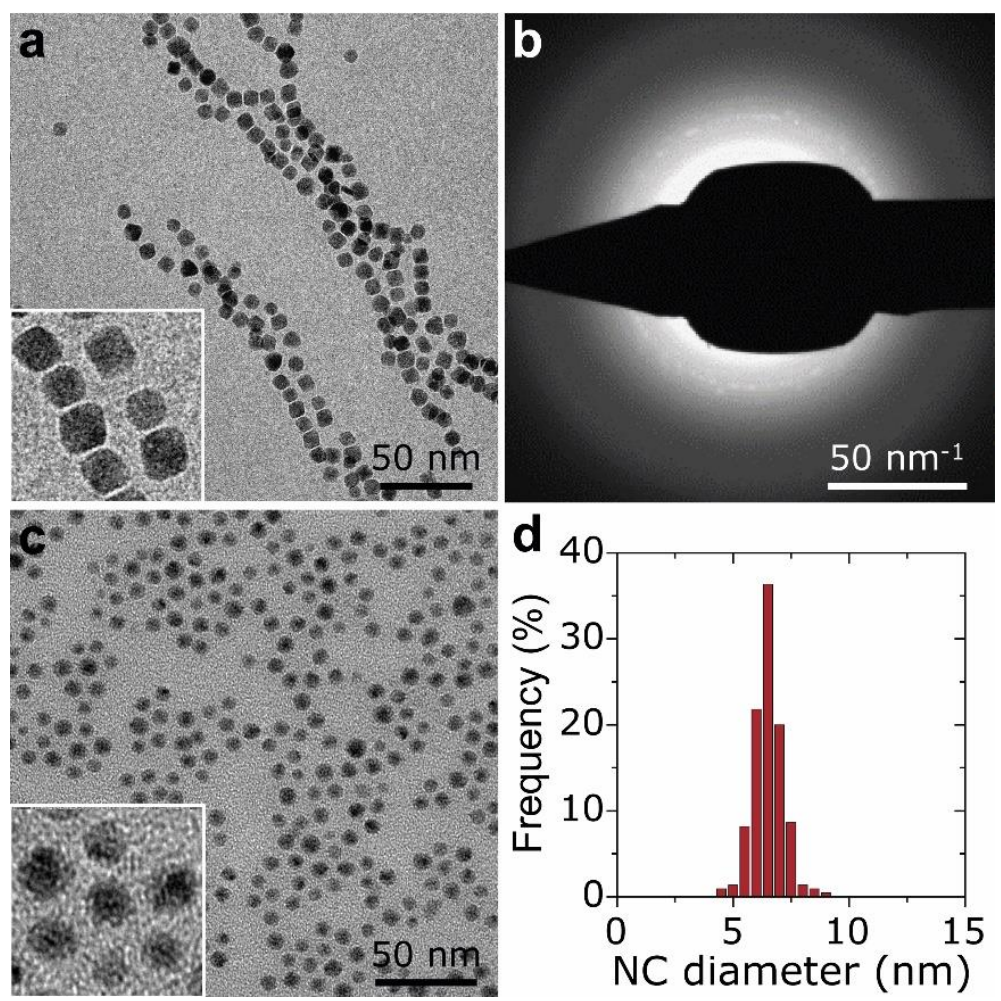

Figure 2.2. Overview of the Co- and CoO-NC. a Cryo-TEM of as-synthesized Co-NC before exposure to air with a higher magnification inset and $\mathbf{b}$ the corresponding electron diffraction pattern. $\mathbf{c}$ TEM of $\mathrm{CoO}-\mathrm{NC}$ with a higher magnification inset and $\mathbf{d}$ size distribution of $\mathrm{CoO}-\mathrm{NC}$ with a surfaceaveraged particle diameter of $6.8 \mathrm{~nm}$.

The average NC sizes and distributions over CNT were investigated using TEM. $\mathrm{Co1} / \mathrm{CNT}$ was non-uniform with isolated $\mathrm{NC}$ as well as 20-60 nm aggregates (Appendix A, Figure A2). The inhomogeneity was believed to result from magnetic interactions between NC consisting of superparamagnetic $\varepsilon$-cobalt, ${ }^{29}$ which prevented the Co-NC from distributing uniformly over the CNT. Werner et al. ${ }^{4}$ reported similar non-uniform distributions of metallic $\mathrm{Co}-\mathrm{NC}$ over silica supports and ascribed this to magnetic interactions of the $\mathrm{Co}-\mathrm{NC}$.

For Co2/CNT, the CoO-NC retained their diameter of 6-7 nm during attachment (Figure 2.3a). High-resolution transmission electron microscopy (HR-TEM) confirmed that the NC were polycrystalline and attached as rather spherical particles onto the CNT (Figure 2.3b). Yang et al. ${ }^{30}$ obtained comparable polycrystalline $\mathrm{CoO}-\mathrm{NC}$ through rapid oxidation at $200^{\circ} \mathrm{C}$ of isolated, monocrystalline NC of hcp-Co. Furthermore, the NC were distributed uniformly over the support, indicating that the magnetic interactions were limited and that diffusion of the NC into the open porous network of the CNT was facile, which is in line with previous observations. ${ }^{3,31}$ Clearly, oxidation of the Co-NC to CoO under air-exposure proved crucial for their uniform distribution over CNT. 

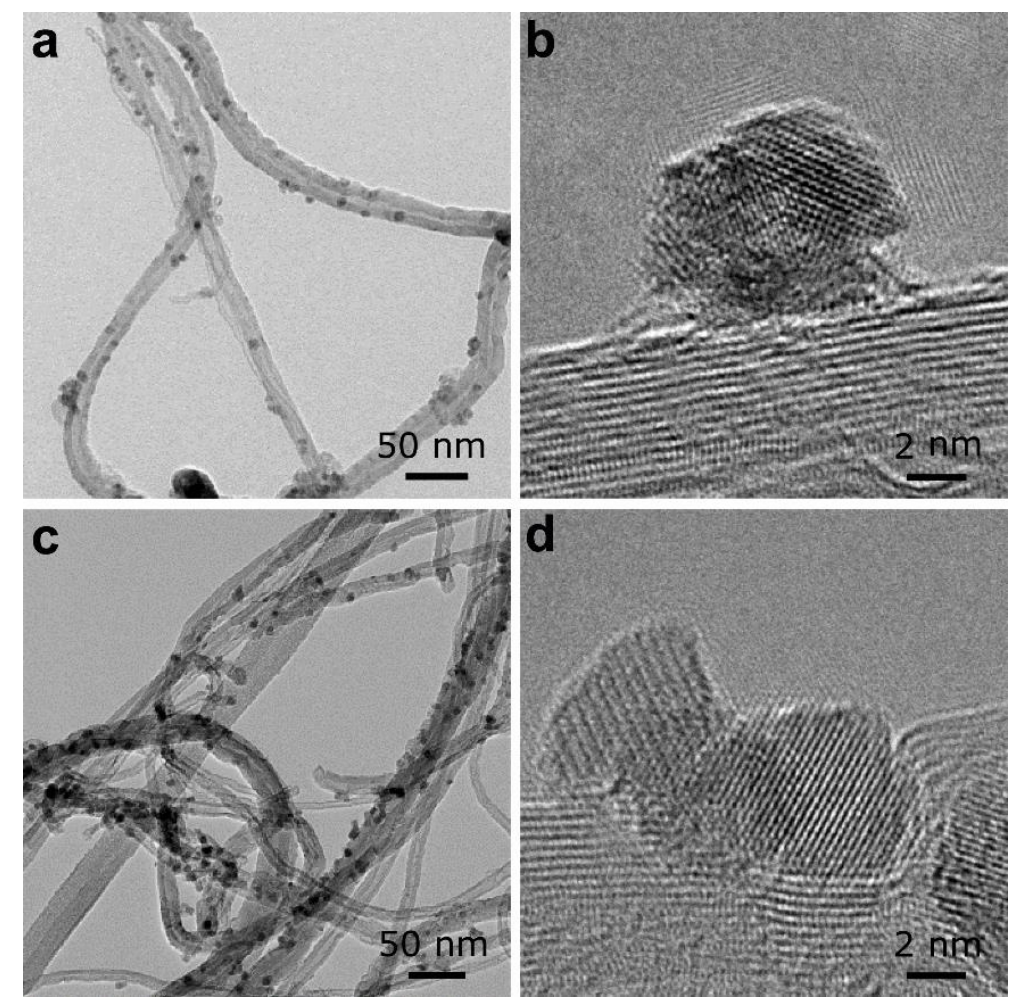

Figure 2.3. Overview of NC supported on CNT. a TEM and b HR-TEM of Co2/CNT, and $\mathbf{c}$ TEM and $\mathbf{d}$ HR-TEM of Co3/CNT.

X-ray diffraction (XRD) pointed to $\varepsilon$-Co crystallites for Co1/CNT (Table 2.1, Appendix A, Figure A3). The average crystallite size of $\sim 6 \mathrm{~nm}$ was in the range of the NC size prior to attachment. XRD analysis of Co2/CNT indicated 2-3 nm crystalline CoO domains, which was consistent with HR-TEM (Figure 2.3b).

Table 2.1. Overview of the prepared catalysts. The metal loading was determined by ICP-OES, the particle diameter $\left(\mathrm{D}[3,2]_{\mathrm{Co}-\mathrm{NC}}\right)$ by TEM and the crystallite size and crystal structure by XRD.

\begin{tabular}{llllll}
\hline Sample & $\begin{array}{c}\text { Oxidation } \\
\text { temp. }\left({ }^{\circ} \mathrm{C}\right)\end{array}$ & $\begin{array}{l}\text { Cobalt } \\
\text { loading } \\
(\mathbf{w t} \%)\end{array}$ & $\begin{array}{l}\mathrm{D} \text { ) } 3,2 \\
(\mathrm{~nm}))^{\mathrm{a}}\end{array}$ & $\begin{array}{l}\text { Co } \mathrm{NC} \\
\text { size }(\mathrm{nm})\end{array}$ & $\begin{array}{l}\text { Crystal } \\
\text { structure }\end{array}$ \\
\hline $\mathrm{Co} 1 / \mathrm{CNT}$ & - & 11 & $7-60$ & $\sim 6$ & $\varepsilon-\mathrm{Co}$ \\
$\mathrm{Co} 2 / \mathrm{CNT}$ & 25 & 8.8 & 5.7 & 2.9 & $\mathrm{CoO}$ \\
$\mathrm{Co} 3 / \mathrm{CNT}$ & 250 & 8.6 & 5.9 & 4.0 & $\mathrm{CO}_{3} \mathrm{O}_{4}$ \\
\hline
\end{tabular}

a Particle size analysis using TEM of NC after attachment to CNT.

To investigate the effect of HT-oxidation, a commonly applied treatment to remove ligands, Co2/CNT was exposed to synthetic air $\left(20 \mathrm{vol} \% \mathrm{O}_{2}\right)$ at $250{ }^{\circ} \mathrm{C}$. The obtained catalyst was labelled Co3/CNT. XRD measurements after the oxidative treatment showed that $\mathrm{CoO}$ was converted into $\mathrm{CO}_{3} \mathrm{O}_{4}$ (Table 2.1, Appendix A, Figure A3). The treatment did not affect the particle size and distribution as both remained largely unchanged (Figure 2.3c, Appendix A, Figure A4). However, at the local scale, HR-TEM showed that the NC now consisted of single crystalline domains although some clustering of $\mathrm{NC}$ and embedding into the CNT was apparent, possibly because of cobalt oxide locally catalyzing oxidation of CNT at high temperature (Figure $2.3 \mathrm{~d}$ ). 


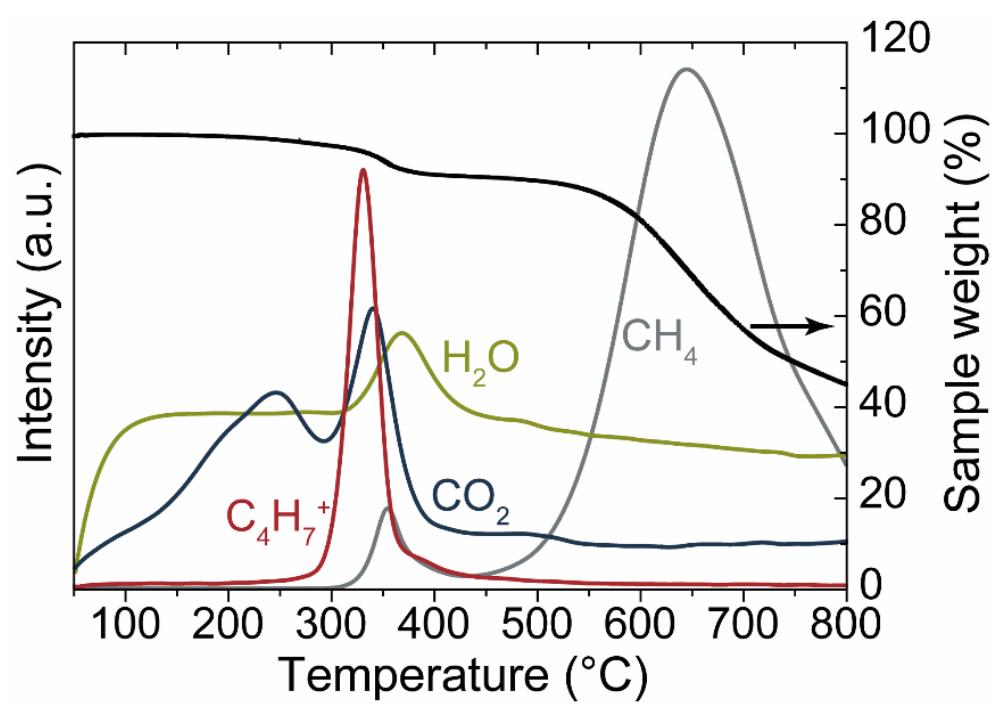

Figure 2.4. Combined TGA and MS results of $\mathrm{Co} 2 / \mathrm{CNT}$ in $25 \mathrm{vol} \% \mathrm{H}_{2}$ with $10{ }^{\circ} \mathrm{C} \cdot \mathrm{min}^{-1}$ heating.

The reduction of the catalysts was investigated using thermogravimetric analysis coupled with mass spectrometry (TGA-MS) in diluted $\mathrm{H}_{2}$. For Co2/CNT, the main weight loss around $650{ }^{\circ} \mathrm{C}$ was associated with cobalt-catalyzed methanation of CNT (Figure 2.4). ${ }^{32}$ At $350{ }^{\circ} \mathrm{C}, \mathrm{CoO}$ was reduced to $\mathrm{Co}^{0}$ as indicated by weight loss and water evolution. Interestingly, $\mathrm{CH}_{4}, \mathrm{CO}_{2}$ and the $\mathrm{C}_{4} \mathrm{H}_{7}^{+}$ion, a fragment ion of oleic acid, were produced as well. These signals were related to desorption and/or decomposition of oleic acid ligands simultaneously with reduction of $\mathrm{CoO}$ at $350{ }^{\circ} \mathrm{C}$. Quantification of the weight loss confirmed that, besides the loss of oxygen from $\mathrm{CoO}$, also ligands were removed. When assuming full reduction of $\mathrm{CoO}, \sim 35 \%$ of the amount of oleic acid added in the NC synthesis was removed during reduction. For $\mathrm{Co} 3 / \mathrm{CNT}$, neither the $\mathrm{C}_{4} \mathrm{H}_{7}{ }^{+}$fragment nor $\mathrm{CH}_{4}$ was observed with TGA-MS and the observed weight loss of $3.8 \%$ matched the theoretical weight loss of $3.1 \%$ (Appendix A, Figure A5). The ligands had thus already been removed in the HT-oxidative treatment.

Table 2.2. Catalytic results obtained for FT at 20 bar, $220{ }^{\circ} \mathrm{C}, \mathrm{H}_{2} / \mathrm{CO}=2 \mathrm{v} / \mathrm{v}, 80-96 \mathrm{~h}$ time-onstream with TOF based on the average particle size of the spent and passivated catalysts $\left(\mathrm{D}[3,2]_{\text {spent }}\right)$ as determined with TEM. The GHSV was $1840 \mathrm{~h}^{-1}$ (Co1/CNT), $7850 \mathrm{~h}^{-1}(\mathrm{Co} 2 / \mathrm{CNT})$ and $5970 \mathrm{~h}^{-1}$ (Co3/CNT) to obtain similar CO conversion levels

\begin{tabular}{|c|c|c|c|c|c|c|}
\hline Sample & $\begin{array}{l}\text { CO conv. } \\
(\%)\end{array}$ & CTY $^{\mathrm{a}}$ & $\begin{array}{l}\text { TOF } \\
\left(10^{-3} \mathrm{~s}^{-1}\right)\end{array}$ & $\begin{array}{l}\mathrm{C}_{1}-\text { sel. } \\
(\% \mathrm{o})\end{array}$ & $\begin{array}{l}\mathrm{C}_{5_{+}}-\text {sel. } \\
\left(\%_{\mathrm{C}}\right)\end{array}$ & $\begin{array}{l}\mathrm{D}[3,2]_{\text {spent }} \\
\text { (nm) }\end{array}$ \\
\hline Co1/CNT & 2.4 & 0.4 & n.a. & n.a. & n.a. & $15-100$ \\
\hline $\mathrm{Co} 2 / \mathrm{CNT}$ & 18 & 22 & 116 & 12 & 78 & 9.0 \\
\hline $\mathrm{Co} 3 / \mathrm{CNT}$ & 15 & 17 & 122 & 9.8 & 82 & 12 \\
\hline
\end{tabular}

${ }^{\mathrm{a}}$ In $10^{-5} \mathrm{~mol}_{\mathrm{CO}} \cdot \mathrm{g}_{\mathrm{Co}}{ }^{-1} \cdot \mathrm{s}^{-1}$.

Co2/CNT was highly active in FT after in situ reduction (Table 2.2, Appendix A, Figure A6). In fact, its cobalt time yield (CTY) was higher than that of a Co/CNT catalyst prepared by Eschemann et al. ${ }^{33}$ via incipient wetness impregnation using a cobalt nitrate precursor solution. The high activity of our Co2/CNT implied that the surface of the cobalt particles was fully accessible to the synthesis gas reactants. Furthermore, during the first 
few hours of FT, substantial activation of the $\mathrm{Co} 2 / \mathrm{CNT}$ could indicate incomplete reduction or residual ligands desorbing from the NC surface due to the formed FT products.

The activity of Co1/CNT was close to zero. This was most likely caused by a large decrease in metallic surface area due to the clustered particles growing together under reaction conditions (Appendix A, Figure A7). Additionally, the lack of washing steps between the synthesis and attachment of the $\mathrm{Co}-\mathrm{NC}$ may have increased the ligand concentration, which in turn may have led to less binding of the Co-NC to the CNT. Casavola et al. ${ }^{3}$ described a similar effect of ligands on the binding of $\mathrm{Fe}-\mathrm{NC}$.

Co3/CNT was significantly less active than Co2/CNT. The HT-oxidative treatment promoted particle growth during in situ reduction and FT. TEM of the spent catalysts revealed a larger particle size for Co3/CNT than for Co2/CNT (Table 2.2, Appendix A, Figure A7). An explanation for the adverse effect of HT-oxidation on the stability of the catalysts could be a different interaction of the NC with the support for Co2/CNT and Co3/CNT, as shown in Figure 2.3. Furthermore, some NC clustering was apparent for the fresh $\mathrm{Co} 3 / \mathrm{CNT}$, which probably led to increased particle growth during reduction and catalysis.

The $\mathrm{C}_{5+}$-selectivity of both Co2/CNT and Co3/CNT was comparable, although Co3/CNT was slightly more selective to $\mathrm{C}_{5+}$-products than Co2/CNT (Table 2.2). For Co1/CNT, no selectivity could be determined owing to its low activity.

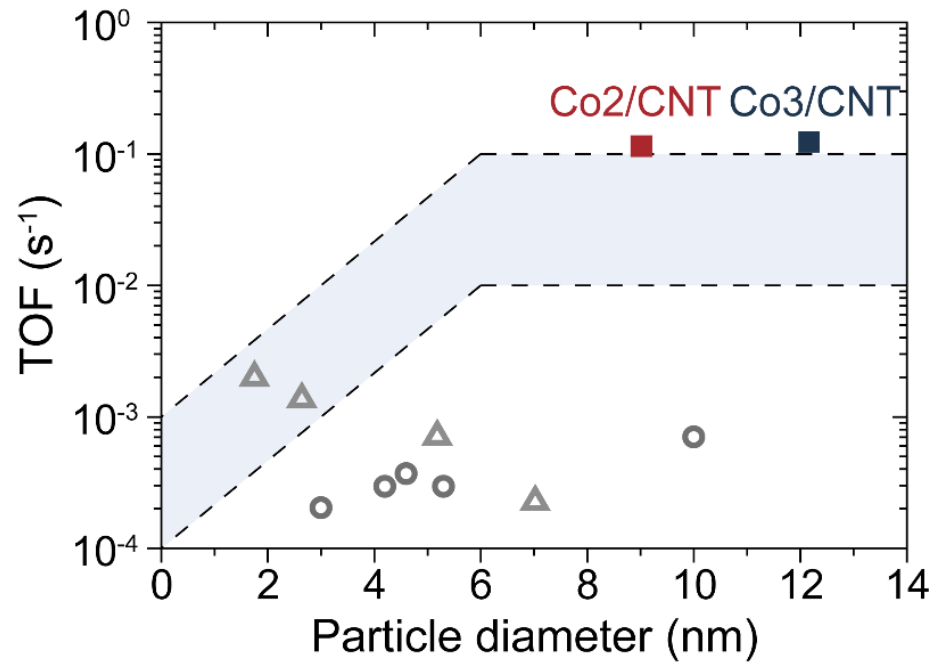

Figure 2.5. Turnover frequencies of $\mathrm{Co} 2 / \mathrm{CNT}$ and $\mathrm{Co} 3 / \mathrm{CNT}$ as a function of the average cobalt particle diameter. Recalculated TOF values of NC-based catalysts for LT-oxidized $\mathrm{Co} / \mathrm{SiO}_{2}$ (grey open circles, ref. ${ }^{22}$ ) and non-oxidized $\mathrm{Co} / \mathrm{TiO}_{2}$ (grey open triangles, ref. ${ }^{23}$ ) are given as comparison. Details of the recalculation can be found in Appendix A. The highlighted area is the typical TOF range of conventional catalysts.

The turnover frequencies (TOF) of Co2/CNT and Co3/CNT were similar (Table 2.2), thereby proving that the higher CTY of $\mathrm{Co} 2 / \mathrm{CNT}$ originated from a larger cobalt surface area instead of higher intrinsic activity. Because the surface of Co3/CNT was free of ligands, as shown before, the surface of the Co2/CNT must have been equally stripped to obtain a 
similar TOF. When comparing the TOF with literature values for LT- or non-oxidized NC catalysts, ${ }^{22,23}$ it is apparent that we obtained more active catalysts, which are now at the high end of the TOF range of conventional catalysts (Figure 2.5). For the HT-oxidized Co3/CNT, full activation and a high TOF was expected, but this is the first time, to the best of our knowledge that only LT-oxidation followed by in situ reduction was sufficient to fully activate a NC-based cobalt catalyst for FT.

\subsection{Conclusion}

In this work, we investigated how oxidative treatments of cobalt nanocrystals (NC) affect their attachment to carbon nanotubes and activation for the Fischer-Tropsch (FT) synthesis. Attachment of $\varepsilon$-cobalt NC resulted in clustering of the NC (probably due to magnetic interactions between the NC) and low activity. After low-temperature oxidation of the $\mathrm{NC}$ to $\mathrm{CoO}$, the NC were attached uniformly. The ligands were readily removed during in situ reduction and high activity was obtained. In fact, it was possible to fully activate a NC-based cobalt catalyst for FT using this mild procedure. High-temperature (HT) oxidation to $\mathrm{Co}_{3} \mathrm{O}_{4}$ led to more pronounced particle growth during in situ reduction or FT and therefore the catalytic activity was lower. An HT-oxidative treatment was thus unnecessary and even counter-productive. These findings will facilitate the application of colloid-based catalysts to investigate structure-performance relationships in catalysis.

\section{Acknowledgements}

The authors acknowledge Shell Global Solutions and the Netherlands Association for Scientific Research (NWO) for funding through the CHIPP program. K.P.d.J. acknowledges the European Research Council, EU FP7 ERC Advanced Grant no. 338846. We thank Peter Munnik for helpful discussions and Paul Bomans (cryo-TEM), Lars van der Wal (HR-TEM), Marjan Versluijs-Helder (TGA-MS) and Helen de Waard (ICP-OES) for the measurements indicated.

\section{References}

1. Zaera, F. Nanostructured materials for applications in heterogeneous catalysis. Chem. Soc. Rev. 42, 2746-2762 (2013).

2. Jia, C.-J. \& Schüth, F. Colloidal metal nanoparticles as a component of designed catalyst. Phys. Chem. Chem. Phys. 13, 2457-2487 (2011).

3. Casavola, M., Hermannsdörfer, J., de Jonge, N., Dugulan, A. I. \& de Jong, K. P. Fabrication of Fischer-Tropsch Catalysts by Deposition of Iron Nanocrystals on Carbon Nanotubes. Adv. Funct. Mater. 25, 5309-5319 (2015).

4. Werner, S., Johnson, G. R. \& Bell, A. T. Synthesis and Characterization of Supported CobaltManganese Nanoparticles as Model Catalysts for Fischer-Tropsch Synthesis. ChemCatChem 6, 2881-2888 (2014).

5. Hondow, N. \& Fuller, R. O. The use of preformed nanoparticles in the production of heterogeneous catalysts. J. Colloid Interface Sci. 417, 396-401 (2014). 
6. Park, J. Y., Aliaga, C., Renzas, J. R., Lee, H. \& Somorjai, G. A. The Role of Organic Capping Layers of Platinum Nanoparticles in Catalytic Activity of CO Oxidation. Catal. Letters 129, 1-6 (2009).

7. Li, D. et al. Surfactant Removal for Colloidal Nanoparticles from Solution Synthesis: The Effect on Catalytic Performance. ACS Catal. 2, 1358-1362 (2012).

8. Lee, I., Morales, R., Albiter, M. A. \& Zaera, F. Synthesis of heterogeneous catalysts with well shaped platinum particles to control reaction selectivity. Proc. Natl. Acad. Sci. U. S. A. 105, 15241-15246 (2008).

9. Iablokov, V. et al. Size-Dependent Activity and Selectivity of Fe/MCF-17 in the Catalytic Hydrogenation of Carbon Monoxide Using Fe(0) Nanoparticles as Precursors. ACS Catal. 6, 2496-2500 (2016).

10. Witte, P. T. et al. BASF NanoSelect ${ }^{\mathrm{TM}}$ Technology: Innovative Supported Pd- and Pt-based Catalysts for Selective Hydrogenation Reactions. Top. Catal. 55, 505-511 (2012).

11. Zacharaki, E. et al. From colloidal monodisperse nickel nanoparticles to well-defined $\mathrm{Ni} / \mathrm{Al}_{2} \mathrm{O}_{3}$ model catalysts. Langmuir 33, 9836-9843 (2017).

12. de Mello Donegá, C. Synthesis and properties of colloidal heteronanocrystals. Chem. Soc. Rev. 40, 1512-1546 (2011).

13. den Breejen, J. P. et al. On the Origin of the Cobalt Particle Size Effects in Fischer-Tropsch Catalysis. J. Am. Chem. Soc. 131, 7197-7203 (2009).

14. Beaumont, S. K. Recent developments in the application of nanomaterials to understanding molecular level processes in cobalt catalysed Fischer-Tropsch synthesis. Phys. Chem. Chem. Phys. 16, 5034-5043 (2014).

15. Liu, S., Li, Y. \& Shen, W. Tuning the catalytic behavior of metal nanoparticles: The issue of the crystal phase. Chinese J. Catal. 36, 1409-1418 (2015).

16. Prieto, G., Martínez, A., Concepción, P. \& Moreno-Tost, R. Cobalt particle size effects in Fischer-Tropsch synthesis: structural and in situ spectroscopic characterisation on reverse micelle-synthesised Co/ITQ-2 model catalysts. J. Catal. 266, 129-144 (2009).

17. Fischer, N., van Steen, E. \& Claeys, M. Structure sensitivity of the Fischer-Tropsch activity and selectivity on alumina supported cobalt catalysts. J. Catal. 299, 67-80 (2013).

18. Lögdberg, S. et al. Effect of water on the space-time yield of different supported cobalt catalysts during Fischer-Tropsch synthesis. Appl. Catal. A Gen. 393, 109-121 (2011).

19. Park, J.-Y. et al. Fischer-Tropsch catalysts deposited with size-controlled $\mathrm{Co}_{3} \mathrm{O}_{4}$ nanocrystals: Effect of Co particle size on catalytic activity and stability. Appl. Catal. A Gen. 411-412, 15-23 (2012).

20. Lee, Y.-J., Park, J.-Y., Jun, K.-W., Wook Bae, J. \& Prasad, P. S. S. Controlled Nanocrystal Deposition for Higher Degree of Reduction in $\mathrm{Co} / \mathrm{Al}_{2} \mathrm{O}_{3}$ Catalyst. Catal. Letters 130, 198-203 (2009).

21. Ralston, W. T., Melaet, G., Saephan, T. \& Somorjai, G. A. Evidence of Structure Sensitivity in the Fischer-Tropsch Reaction on Model Cobalt Nanoparticles by Time-Resolved Chemical Transient Kinetics. Angew. Chemie Int. Ed. 56, 7415-7419 (2017).

22. Herranz, T., Deng, X., Cabot, A., Guo, J. \& Salmeron, M. Influence of the Cobalt Particle Size in the CO Hydrogenation Reaction Studied by In Situ X-Ray Absorption Spectroscopy. J. Phys. Chem. B113, 10721-10727 (2009). 
23. Delgado, J. A. et al. Fischer-Tropsch synthesis catalysed by small $\mathrm{TiO}_{2}$ supported cobalt nanoparticles prepared by sodium borohydride reduction. Appl. Catal. A Gen. 513, 39-46 (2016).

24. Delgado, J. A. et al. Effect of polymeric stabilizers on Fischer-Tropsch synthesis catalyzed by cobalt nanoparticles supported on $\mathrm{TiO}_{2}$. J. Mol. Catal. A Chem. 417, 43-52 (2016).

25. Sonström, P. \& Bäumer, M. Supported colloidal nanoparticles in heterogeneous gas phase catalysis: on the way to tailored catalysts. Phys. Chem. Chem. Phys. 13, 19270-19284 (2011).

26. Iablokov, V. et al. Size-Controlled Model Co Nanoparticle Catalysts for $\mathrm{CO}_{2}$ Hydrogenation: Synthesis, Characterization, and Catalytic Reactions. Nano Lett. 12, 3091-3096 (2012).

27. Reuel, R. C. \& Bartholomew, C. H. The Stoichiometries of $\mathrm{H}_{2}$ and $\mathrm{CO}$ Adsorptionson Cobalt: Effects of Support and Preparation. J. Catal. 85, 63-77 (1984).

28. Dinega, D. P. \& Bawendi, M. G. A Solution-Phase Chemical Approach to a New Crystal Structure of Cobalt. Angew. Chemie Int. Ed. 38, 1788-1791 (1999).

29. Gross, A. F., Diehl, M. R., Beverly, K. C., Richman, E. K. \& Tolbert, S. H. Controlling Magnetic Coupling between Cobalt Nanoparticles through Nanoscale Confinement in Hexagonal Mesoporous Silica. J. Phys. Chem. B 107, 5475-5482 (2003).

30. Yang, Z., Lisiecki, I., Walls, M. \& Pileni, M.-P. Nanocrystallinity and the Ordering of Nanoparticles in Two-Dimensional Superlattices: Controlled Formation of Either Core/Shell (Co/CoO) or Hollow CoO Nanocrystals. ACS Nano 7, 1342-1350 (2013).

31. Seo, B. et al. Size-Dependent Activity Trends Combined with in Situ X-ray Absorption Spectroscopy Reveal Insights into Cobalt Oxide/Carbon Nanotube-Catalyzed Bifunctional Oxygen Electrocatalysis. ACS Catal. 6, 4347-4355 (2016).

32. Xiong, H., Motchelaho, M. A. M., Moyo, M., Jewell, L. L. \& Coville, N. J. Cobalt catalysts supported on a micro-coil carbon in Fischer-Tropsch synthesis: A comparison with CNTs and CNFs. Catal. Today 214, 50-60 (2013).

33. Eschemann, T. O. et al. Effect of support surface treatment on the synthesis, structure, and performance of Co/CNT Fischer-Tropsch catalysts. J. Catal. 328, 130-138 (2015). 
Assembly and activation of supported cobalt nanocrystal catalysts

for the Fischer-Tropsch synthesis 


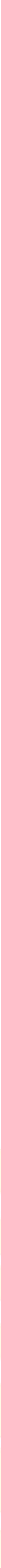




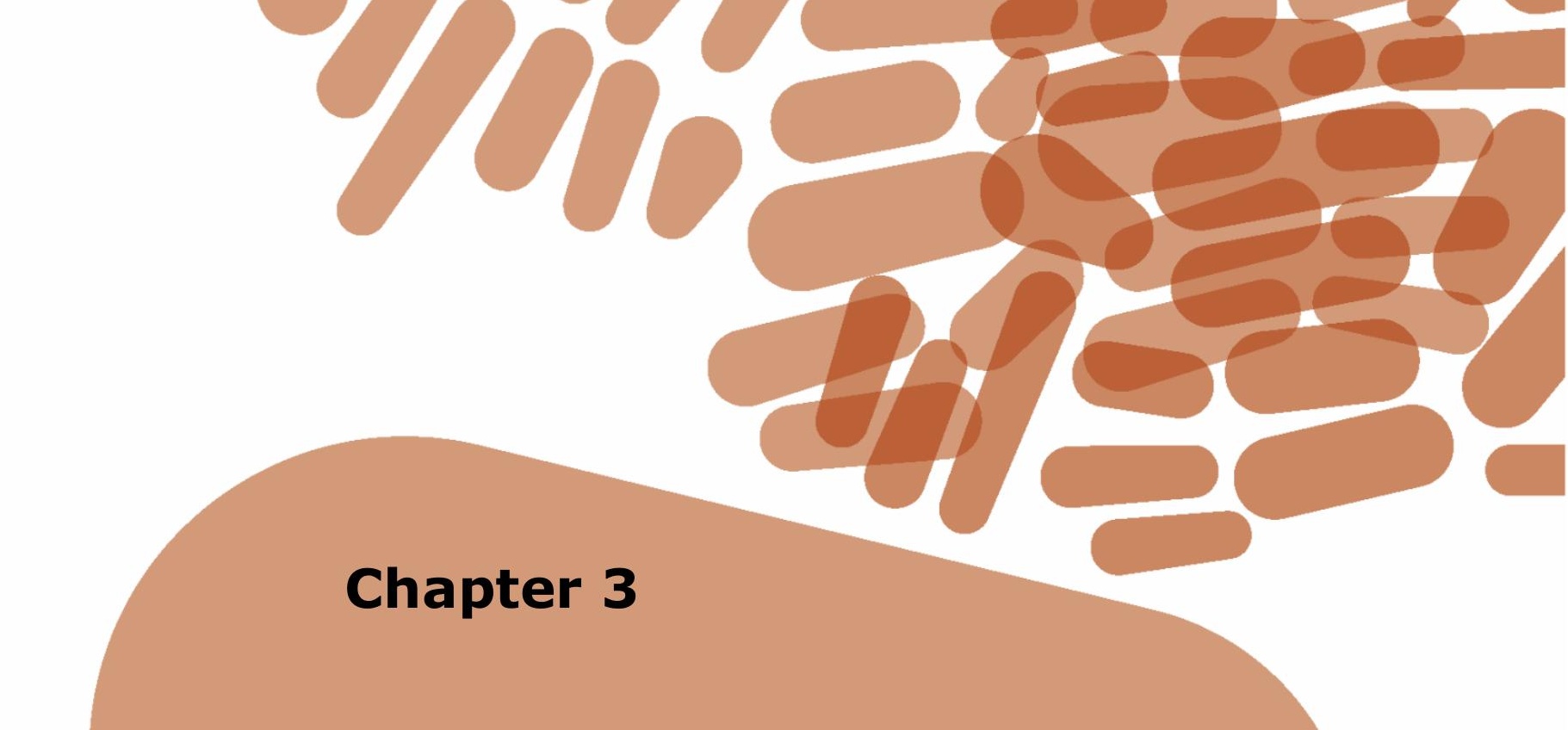

Cobalt nanocrystals on carbon nanotubes in the Fischer-Tropsch synthesis: impact of support oxidation

\begin{abstract}
In this chapter, we investigated the effect of oxidation of a carbon nanotube (CNT) support on the structure and catalytic performance in the Fischer-Tropsch (FT) synthesis of presynthesized cobalt nanocrystals $(\mathrm{NC})$. CoO-NC of $6 \mathrm{~nm}$ were prepared using colloidal techniques and impregnated on pristine and surface-oxidized CNT (10 wt \% Co). During reduction, the $\mathrm{CoO}-\mathrm{NC}$ on pristine $\mathrm{CNT}$ were converted completely to Co while growing to $11 \mathrm{~nm}$ on average. In contrast, on oxidized CNT (oxCNT), only 50\% reduction of CoO was observed and average NC growth was absent, indicating a stronger Co-support interaction on oxCNT than on CNT. In FT, the turnover frequency (TOF) after $100 \mathrm{~h}$ on stream was exceptionally high on pristine $\mathrm{CNT}\left(150 \cdot 10^{-3} \mathrm{~s}^{-1}\right)$ and a factor of two lower on oxCNT $(63 \cdot 10$ ${ }^{3} \mathrm{~s}^{-1}$ ). In the initial stage of FT, the TOF was $35 \%$ lower on oxCNT than on CNT, which matched well with the $32 \%$ lower crystallinity of metallic cobalt during FT determined using in situ XRD. Furthermore, deactivation was observed mainly on oxCNT while NC growth, restructuring, or oxidation during FT were excluded, leaving enhanced deposition of carbonaceous species on $\mathrm{Co}-\mathrm{NC} / \mathrm{oxCNT}$ as possible causes. These results demonstrate the large impact of the support on the structure and performance of well-defined catalysts based on cobalt nanocrystals.
\end{abstract}

Based on: van Deelen, T. W., Yoshida, H., Oord, R., Zečević, J., Weckhuysen, B. M. \& de Jong, K. P. Appl. Catal. A Gen., DOI: 10.1016/j.apcata.2020.117441 


\subsection{Introduction}

The most frequently applied supports for cobalt-based Fischer-Tropsch (FT) synthesis catalysts are oxidic compounds, such as $\mathrm{Al}_{2} \mathrm{O}_{3}, \mathrm{SiO}_{2}$ or $\mathrm{TiO}_{2}$, which usually interact with cobalt and influence the FT performance. ${ }^{1}$ Carbon supports, on the other hand, present an interesting alternative, especially for fundamental studies, given their chemical inertness (limited or no cobalt-support compound formation).Various types of carbon have been applied as supports, ${ }^{2}$ including activated carbon, ordered mesoporous carbon, ${ }^{3}$ and carbon nanofibers ${ }^{4,5}$ and carbon nanotubes. ${ }^{6}$ Among these, carbon nanotubes (CNT) have an open, macro- and mesoporous structure combined with a high specific surface area. ${ }^{7}$ Moreover, the surface of pristine CNT contains a low amount of functional groups, rendering these hydrophobic. ${ }^{8}$ To increase hydrophilicity, oxygen-containing functional groups can be introduced by oxidation of pristine CNT, for example using nitric and/or sulfuric acid. ${ }^{8,9}$ Functional groups can also act as anchoring sites for metal nanoparticles and hence, metals supported on oxidized CNT (oxCNT) typically display increased resistance towards sintering under reaction conditions. ${ }^{10}$

Several groups reported higher activity for Co/oxCNT compared to Co/CNT, while $\mathrm{C}_{5_{+}-}$ selectivity decreased with CNT oxidation. ${ }^{11-13}$ Interestingly, Eschemann et al. ${ }^{6}$ showed that CNT functionalization has a major effect on the FT performance of Co/CNT catalysts, with significantly lower activity and $\mathrm{C}_{5_{+}}$-selectivity for oxCNT. The different catalytic performance was tentatively ascribed to an increase in hcp Co on pristine CNT compared to oxCNT, as observed by in situ XRD during reduction, because often hcp Co is more active in FT than fcc Co. ${ }^{14-18}$ However, the exact nature of the differences between Co supported on CNT and oxCNT was not established.

More insight into the metal-support interaction after support functionalization and its effect in FT could potentially be obtained by using better defined catalysts in combination with advanced characterization techniques. Colloidal techniques offer the possibility to synthesize metal nanocrystals (NC) separately with high precision in e.g. NC size, shape or crystal structure. ${ }^{19}$ In the case of $\mathrm{Co}-\mathrm{NC}$, low-temperature oxidation to CoO facilitates their subsequent uniform attachment and ligand removal during reduction. ${ }^{20}$ Because $\mathrm{NC}$ synthesis is decoupled from attachment to the support, the starting cobalt particles should be very similar, meaning that differences in FT must be caused by altered metal-support interactions related to functionalization of the CNT.

In this chapter, we investigated the effect of CNT surface oxidation on the structure and catalytic performance of CNT-supported cobalt nanocrystals in the Fischer-Tropsch synthesis. Catalyst were prepared via colloidal synthesis of $6 \mathrm{~nm} \mathrm{CoO}-\mathrm{NC}$ and subsequent attachment to CNT and oxCNT. CNT oxidation resulted in decreased reducibility and sintering propensity of the cobalt (oxide) phase during reduction. In FT, Co-NC on pristine CNT were highly active, while the activity on oxCNT was approximately a factor two lower. Using in situ XRD, the crystalline metallic Co content on oxCNT was found to be $32 \%$ lower than on pristine CNT during 40 , making it the most probable cause for the observed $35 \%$ lower initial TOF on oxCNT. Catalyst deactivation during FT occurred mainly on oxCNT, while particle growth, NC restructuring, or oxidation were excluded as deactivation mechanisms, leaving enhanced carbon deposition as a likely option. 


\subsection{Experimental}

\subsubsection{Oxidation of carbon nanotubes}

Oxidized CNT (oxCNT) were obtained by heating $4 \mathrm{~g}$ CNT (Baytubes c150 HP) in $150 \mathrm{~mL}$ concentrated $\mathrm{HNO}_{3}$ at $80{ }^{\circ} \mathrm{C}$ for $90 \mathrm{~min}$. Afterwards, the oxCNT was washed on a vacuum filtration setup with $2.5 \mathrm{~L}$ deionized $\mathrm{H}_{2} \mathrm{O}$ until the $\mathrm{pH}$ of the filtrate reached 5 . The washed oxCNT was dried at $120^{\circ} \mathrm{C}$ in static air overnight.

\subsubsection{Synthesis of cobalt nanocrystals}

The colloidal synthesis of cobalt nanocrystals (Co-NC) was based on a previously reported method, ${ }^{21}$ which was selected because the use of phosphorus-containing ligands was avoided. The synthesis was performed under $\mathrm{N}_{2}$ atmosphere on a Schlenk line. Typically, $65 \mathrm{mg}$ of oleic acid (Sigma-Aldrich, $90 \%$, technical grade) was transferred to a $100 \mathrm{~mL} 3-$ necked flask equipped with a cooler and two septa and evacuated at $100{ }^{\circ} \mathrm{C}$ for $30 \mathrm{~min}$. After flushing several times with $\mathrm{N}_{2}, 7.5 \mathrm{~mL}$ of 1,2-dichlorobenzene (Sigma-Aldrich, $99 \%$, anhydrous) was transferred under $\mathrm{N}_{2}$ to the flask and the mixture was heated to $174{ }^{\circ} \mathrm{C}$ under $750 \mathrm{rpm}$ magnetic stirring. Meanwhile, $270 \mathrm{mg}$ dicobalt octacarbonyl (Acros Organics, $95 \%$, stabilized) was dissolved in $1.5 \mathrm{~mL} \mathrm{1,2-dichlorobenze} \mathrm{inside} \mathrm{a} \mathrm{glovebox.}$ When the solution in the flask reached $174^{\circ} \mathrm{C}$, the cobalt precursor was rapidly injected into the flask. The solution was left to react for 20 minutes and quenched afterwards using a water bath. The Co-NC suspension was exposed to air for one hour at room temperature under $650 \mathrm{rpm}$ magnetic stirring to convert $\mathrm{Co}-\mathrm{NC}$ to $\mathrm{CoO}-\mathrm{NC}$. Subsequently, the colloidal $\mathrm{CoO}$ dispersion was transferred to glass centrifuge tubes, filled up to $20 \mathrm{~mL}$ with 2 propanol (Sigma-Aldrich, >99\%, LC-MS Chromasolv) and centrifuged at $2200 \mathrm{G}$ for 20 minutes. The supernatant was decanted and the precipitated $\mathrm{CoO}-\mathrm{NC}$ were redispersed in n-hexane (Acros Organics, 99+\%) using sonication. This washing cycle with 2-propanol was repeated 3 times more and finally the NC were re-dispersed in $2 \mathrm{~mL}$ toluene (Acros Organics, 99+\%, extra pure) and stored in a glass vial.

\subsubsection{Attachment of cobalt oxide nanocrystals on CNT and oxCNT}

The supports were sieved to a grain size of 75-150 $\mu \mathrm{m}$ prior to being used. Attachment of $10 \mathrm{wt} \%$ of $\mathrm{CoO}-\mathrm{NC}$ on CNT and oxCNT was performed by pore-filling impregnation with the colloidal dispersion. For this, the (ox)CNT were dried at $80{ }^{\circ} \mathrm{C}$ under vacuum for $1 \mathrm{~h}$. After cooling to room temperature, $2.5 \mathrm{~mL} \cdot \mathrm{g}_{\text {support }}{ }^{-1}$ of colloidal dispersion in toluene, equivalent to the pore volume of (ox)CNT, was injected to the support under magnetic stirring. The toluene evaporated upon contact with the vacuum, immediately emptying the syringe. After mixing, the sample was dried at $60^{\circ} \mathrm{C}$ for $1 \mathrm{~h}$ in static air, $120{ }^{\circ} \mathrm{C}$ for $3 \mathrm{~h}$ in static air and $80^{\circ} \mathrm{C}$ for $3 \mathrm{~h}$ under vacuum.

\subsubsection{Characterization}

CNT and oxCNT were titrated using a Radiometer Analytical TitraLab TIM880 automatic titration setup. Between 25-30 mg of sample was suspended in $30 \mathrm{~mL} 0.1 \mathrm{M} \mathrm{KCl}$ by sonication, flushed for 10 min with $\mathrm{N}_{2}$ to remove $\mathrm{CO}_{2}$ and subsequently titrated with $0.01 \mathrm{M}$ 
$\mathrm{NaOH}$ in $0.1 \mathrm{M} \mathrm{KCl}$ under $\mathrm{N}_{2}$ atmosphere. The data was analyzed as described by Eschemann et al. ${ }^{6}$, i.e. a second-degree equation $\left(y=a x^{2}+b x+c\right)$ was fitted to the first derivative of the $\mathrm{pH}$ curve and the inflection point was calculated using $x_{\text {inflection }}=-b / 2 \mathrm{a}$.

$\mathrm{N}_{2}$-physisorption was measured on a Micromeritics Tristar 3000. The samples were pre-dried under $\mathrm{N}_{2}$ flow at $120{ }^{\circ} \mathrm{C}$ for $80 \mathrm{~h}$ for CNT and $150{ }^{\circ} \mathrm{C}$ for $20 \mathrm{~h}$ for oxCNT. Both samples were analyzed over $\mathrm{P} / \mathrm{P}_{0}=0-0.995$ at $-196{ }^{\circ} \mathrm{C}$. The surface area was determined using the BET method and the mesopore volume by single point analysis of the desorption isotherm at $\mathrm{P} / \mathrm{P}_{0}=0.995$.

Transmission electron microscopy (TEM) was performed on an FEI Tecnai 12 operating at $120 \mathrm{kV}$. Scanning transmission electron microscopy with energy-dispersive $\mathrm{X}$-ray spectroscopy (STEM-EDX) was performed in high-angle annular dark-field (HAADF) mode on a Talos F200X (Thermo Fisher Scientific). The microscope was equipped with a high-brightness field emission gun (X-FEG) and a Super-X G2 EDX detector and was operated at $200 \mathrm{kV}$. Samples of colloidal dispersions in hexane were prepared by drop casting on a carbon coated $\mathrm{Cu}$ grid. In the case of supported catalysts, the powder was first suspended in 2-propanol by sonication and subsequently drop casted on a holey carbon coated $\mathrm{Cu}$ grid. The particle sizes were manually determined using iTEM software and at least 200 particles were analyzed to allow a statistical analysis. All reported particle sizes were recalculated to equivalent metallic Co size by correcting for the lattice expansion of a $3 \mathrm{~nm} \mathrm{CoO}$ passivation layer (Co-NC and reduced/spent catalysts). All particle sizes are reported as volume/surface mean diameter $(\mathrm{D}[3,2])$.

Inductively coupled plasma-optical emission spectroscopy (ICP-OES) was measured on a SPECTRO ARCOS and samples were prepared by cobalt extraction in aqua regia.

Temperature programmed reduction (TPR) was measured using a Micromeritics AutoChem 2920. $25 \mathrm{mg}$ of sample was reduced by heating to $800{ }^{\circ} \mathrm{C}$ with $5{ }^{\circ} \mathrm{C} \cdot \mathrm{min}^{-1}$ in a flow of $5 \mathrm{vol} \% \mathrm{H}_{2}$ in $\mathrm{Ar}$.

$\mathrm{X}$-ray diffraction (XRD) was performed on a Bruker D2 Phaser using Co radiation (K $\alpha 1$ $1.789 \AA)$. The diffractograms were analyzed and fitted using Bruker's Eva and Topas software. After fitting the full scan from $20-100^{\circ} 2 \theta$, the crystallite size was calculated using the Scherrer equation to analyze the $\mathrm{CoO}[111]$ peak.

In situ XRD patterns were measured on a Bruker D8 Discover with a Mo (Ko1 $0.709 \AA)$ source in Debye-Scherrer transmission (capillary) geometry. The $\mathrm{X}$-ray beam was focused on a quartz capillary with $1000 \mu \mathrm{m}$ OD and a wall thickness of $10 \mu \mathrm{m}$ using a Göbel-mirror. The setup was equipped with an energy dispersive LynxEye XE Position Sensitive Detector (PSD). Details on the complete setup can be found in recent publications. ${ }^{22,23}$ The patterns were measured between 9 and $39^{\circ} 2 \theta$ with an increment of $0.07^{\circ}$ and time per step of $15.3 \mathrm{~s}$. Multiple scans were accumulated to improve the signal-to-noise ratio only if the patterns were identical. Rietveld Quantitative Phase Analysis (Rietveld QPA) was performed on the measured diffractograms using Bruker TOPAS (v5) software. Details of the Rietveld refinement procedure are given in Appendix B. Typically, $3.5 \mathrm{mg}$ sample in the sieve fraction 75-150 $\mu \mathrm{m}$ was loaded in the capillary and fixed with quartz wool at either end, resulting in a catalyst bed length of $\sim 20 \mathrm{~mm}$. The capillary was placed in the setup and the catalysts were reduced in situ at $350{ }^{\circ} \mathrm{C}$ for $8 \mathrm{~h}\left(1{ }^{\circ} \mathrm{C} \cdot \mathrm{min}^{-1}\right)$ in a $1.2 \mathrm{~mL} \cdot \mathrm{min}^{-1}$ flow of 25 
vol $\% \mathrm{H}_{2}$ in He at 1 bar. After reduction, the temperature was lowered to $180{ }^{\circ} \mathrm{C}$ with $3{ }^{\circ} \mathrm{C} \cdot \mathrm{min}^{-1}$ and at this temperature, a $3.0 \mathrm{~mL} \cdot \mathrm{min}^{-1}$ flow of synthesis gas $\mathrm{H}_{2} / \mathrm{CO}=2(\mathrm{v} / \mathrm{v})$ was introduced. After $10 \mathrm{~min}$ stabilization, the back-pressure regulator was set to $10.6 \mathrm{bar}$. Only after the pressure was reached and stable, the temperature was increased to $220{ }^{\circ} \mathrm{C}$ with $1^{\circ} \mathrm{C} \cdot \mathrm{min}^{-1}$, which was defined as TOS $=0 \mathrm{~h}$. In situ XRD patterns were recorded both during reduction and FT.

\subsubsection{Fischer-Tropsch synthesis}

The performance of the catalysts was evaluated using an Avantium Flowrence 16 parallel reactor setup. Stainless steel plug-flow reactors $(2.6 \mathrm{~mm} \mathrm{ID)}$ were loaded with a mixture of

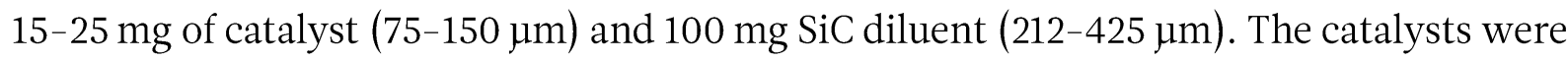
reduced in situ at $350{ }^{\circ} \mathrm{C}$ for $8 \mathrm{~h}\left(1{ }^{\circ} \mathrm{C} \cdot \mathrm{min}^{-1}\right)$ in $25 \mathrm{vol} \% \mathrm{H}_{2}$ in He flow at 1 bar. Subsequently, the reactors were cooled to $180^{\circ} \mathrm{C}$ and the pressure was increased to 20 bar under $\mathrm{H}_{2}$ flow. Synthesis gas with $\mathrm{H}_{2} / \mathrm{CO}=2(\mathrm{v} / \mathrm{v}), 5 \mathrm{vol} \% \mathrm{He}$ as internal standard and GHSV $=5950-$ $8900 \mathrm{~h}^{-1}$ was introduced and the reactors were heated to $220^{\circ} \mathrm{C}$ with $1{ }^{\circ} \mathrm{C} \cdot \mathrm{min}^{-1}$. The reaction products were analyzed online with an Agilent 7890A GC. The hydrocarbon products were separated on an Agilent J\&W PoraBOND Q column and analyzed by an FID. The permanent gasses were separated on a ShinCarbon ST column and quantified using a TCD. Catalytic activity was reported as CO conversion $\left(\mathrm{X}_{\mathrm{CO}}\right)$, cobalt-time yield (CTY) and turnover frequency (TOF). The selectivity (in \%c) to $\mathrm{C}_{1}-\mathrm{C}_{4}$ hydrocarbons was determined as $\mathrm{S}_{\mathrm{C} 1-\mathrm{C} 4}=100 \cdot \mathrm{F}_{\mathrm{Cn}} \cdot \mathrm{n} \cdot\left(\mathrm{F}_{\mathrm{CO}, \text { in }} \cdot \mathrm{X}_{\mathrm{CO}}\right)^{-1}$ with $\mathrm{n}$ being the carbon number of the products and $\mathrm{F}$ the flow of the hydrocarbon or $\mathrm{CO}$. The selectivity to $\mathrm{C}_{5_{+}}$hydrocarbons was calculated as $\mathrm{S}_{\mathrm{C} 5+}=100 \% \mathrm{C}^{-} \mathrm{S}_{\mathrm{C} 1-4}$.

\subsection{Results and discussion}

\subsubsection{Oxidation of carbon nanotubes}

Pristine CNT were oxidized using nitric acid to introduce O-containing functional groups and defects in the CNT surface. An increase in acidic groups was found (Table 3.1) with values similar to what has been obtained previously in our group using gas phase oxidation ( $\sim 0.5 \mathrm{mmol} \cdot \mathrm{g}^{-1}$ acidic groups). ${ }^{6}$ In addition, the structure of CNT and oxCNT was investigated using $\mathrm{N}_{2}$-physisorption (Table 3.1, isotherms in Appendix B, Figure B1). The specific surface area increased by $50 \mathrm{~m}^{2} \cdot \mathrm{g}^{-1}$ as a result of the functionalization, probably due to surface roughening and the creation of defects. The pore volume, determined at $\mathrm{p} / \mathrm{p}_{0}=0.995$, was somewhat lower for oxCNT. Overall, these results were well in line with previous reports. ${ }^{6,13}$

Table 3.1. Structural properties of the pristine and functionalized CNT. The number of acidic groups was determined by titration and the structural properties of the CNT by $\mathrm{N}_{2}$-physisorption.

\begin{tabular}{llll}
\hline Support material & $\begin{array}{l}\text { Acidic groups } \\
\left(\mathrm{mmol} \cdot \mathrm{g}^{-1}\right)\end{array}$ & $\begin{array}{l}\text { BET } \\
\left(\mathrm{m}^{2} \cdot \mathrm{g}^{-1}\right)\end{array}$ & $\begin{array}{l}\text { Pore volume } \\
\left(\mathrm{mL} \cdot \mathrm{g}^{-1}\right)\end{array}$ \\
\hline CNT & 0.02 & 198 & 1.5 \\
OxCNT & 0.72 & 248 & 1.3 \\
\hline
\end{tabular}




\subsubsection{Synthesis of cobalt oxide nanocrystals}

Metallic Co-NC were synthesized, exposed to air to allow oxidation to $\mathrm{CoO}$ and analyzed using TEM (Figure 3.1a,b). The NC were rather spherical and the surface-volume averaged size $(\mathrm{D}[3,2])$ of the $\mathrm{NC}$ was consistently between 6-7 $\mathrm{nm}$ with a standard deviation below $1 \mathrm{~nm}$.

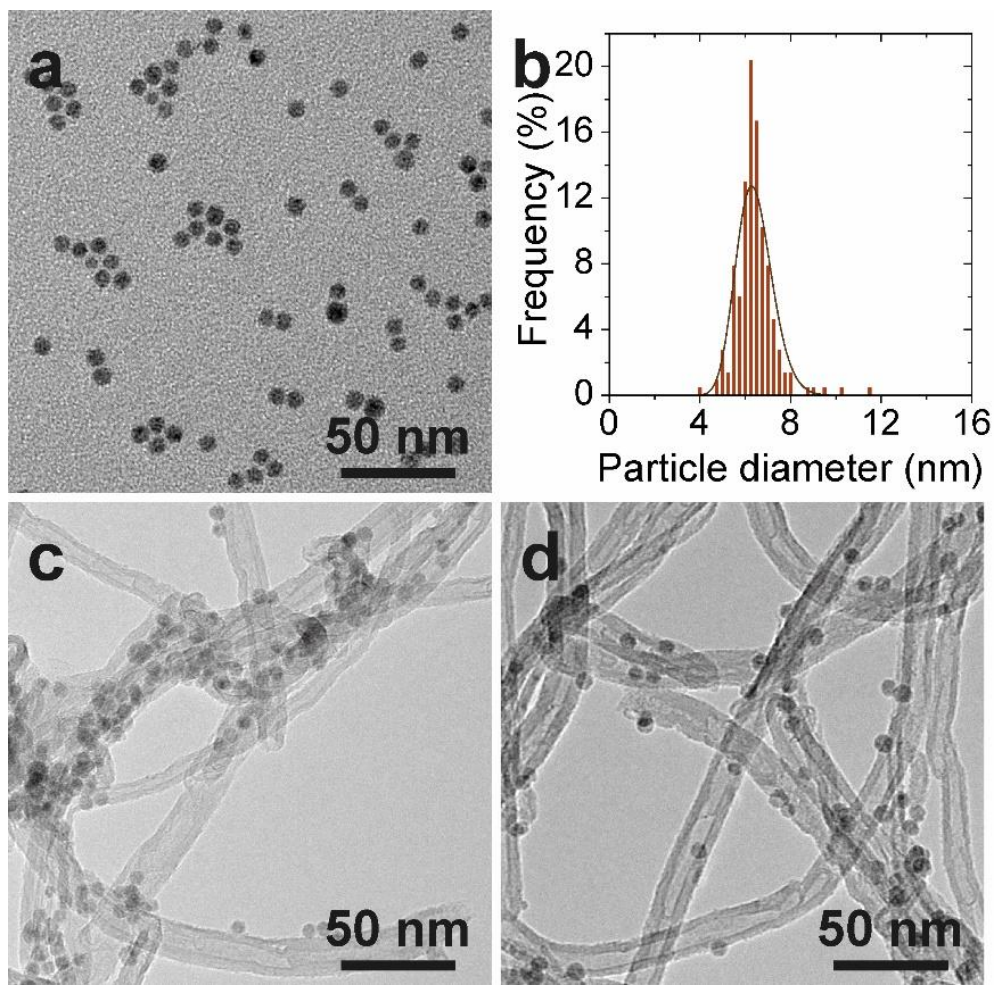

Figure 3.1. TEM results of as-synthesized $\mathrm{CoO}-\mathrm{NC}$ and the CNT- and oxCNT supported samples after $\mathrm{NC}$ attachment and drying. a TEM image of $\mathrm{CoO}-\mathrm{NC}$ with a Co-equivalent surface-volume averaged size $\left(\mathrm{D}[3,2]_{\mathrm{Co}}\right)$ of $6.5 \pm 0.9 \mathrm{~nm}$ and $\mathbf{b}$ the corresponding particle size distribution with lognormal distribution. TEM images of CoO-NC deposited on $\mathbf{c}$ CNT and on $\mathbf{d}$ oxCNT.

\subsubsection{Catalyst preparation}

Model catalysts were prepared by attaching CoO-NC to CNT and oxCNT through impregnation with a colloidal suspension. The cobalt loading of the catalysts was $\sim 10 \mathrm{wt} \%$, as determined by ICP-OES (Table 3.2), and the catalysts were imaged by TEM (Figure 3.1cd). The CoO-NC were uniformly distributed over oxCNT and appeared somewhat more clustered on CNT. In both cases, the NC were predominantly located on the external surface of the CNT. Analysis of the surface-volume averaged particle sizes (Table 3.2) revealed that the $\mathrm{CoO}-\mathrm{NC}$ sizes of 6-7 $\mathrm{nm}$ was unchanged after attachment.

The crystal structure of the NC was investigated with XRD (Figure 3.2a, Table 3.2). The 6-7 nm NC consisted of multiple CoO crystallite domains of $2.2 \mathrm{~nm}$ on average, which was caused by the low-temperature oxidation treatment directly after their synthesis, as shown before. ${ }^{20}$ 
Table 3.2. Properties of the as-prepared samples.

\begin{tabular}{llll}
\hline Sample & $\begin{array}{l}\text { Metal loading } \\
(\mathbf{w t} \%)^{\mathrm{a}}\end{array}$ & $\mathrm{D}[3,2]_{\mathrm{co}}(\mathrm{nm})^{\mathrm{b}}$ & $\begin{array}{l}\text { Average crystallite } \\
\text { size }(\mathrm{nm})^{\mathrm{c}}\end{array}$ \\
\hline $\mathrm{CoO}-\mathrm{NC} / \mathrm{CNT}$ & 9.7 & $5.9 \pm 1.0$ & $2.2(\mathrm{CoO})$ \\
$\mathrm{CoO}-\mathrm{NC} /$ oxCNT & 9.6 & $6.7 \pm 1.3$ & $2.2(\mathrm{CoO})$ \\
\hline
\end{tabular}

${ }^{a}$ Metal loading determined with ICP-OES.

${ }^{b}$ Particle size analysis from TEM of NC after attachment to (ox)CNT.

${ }^{\mathrm{c}}$ Average crystallite size derived from XRD.

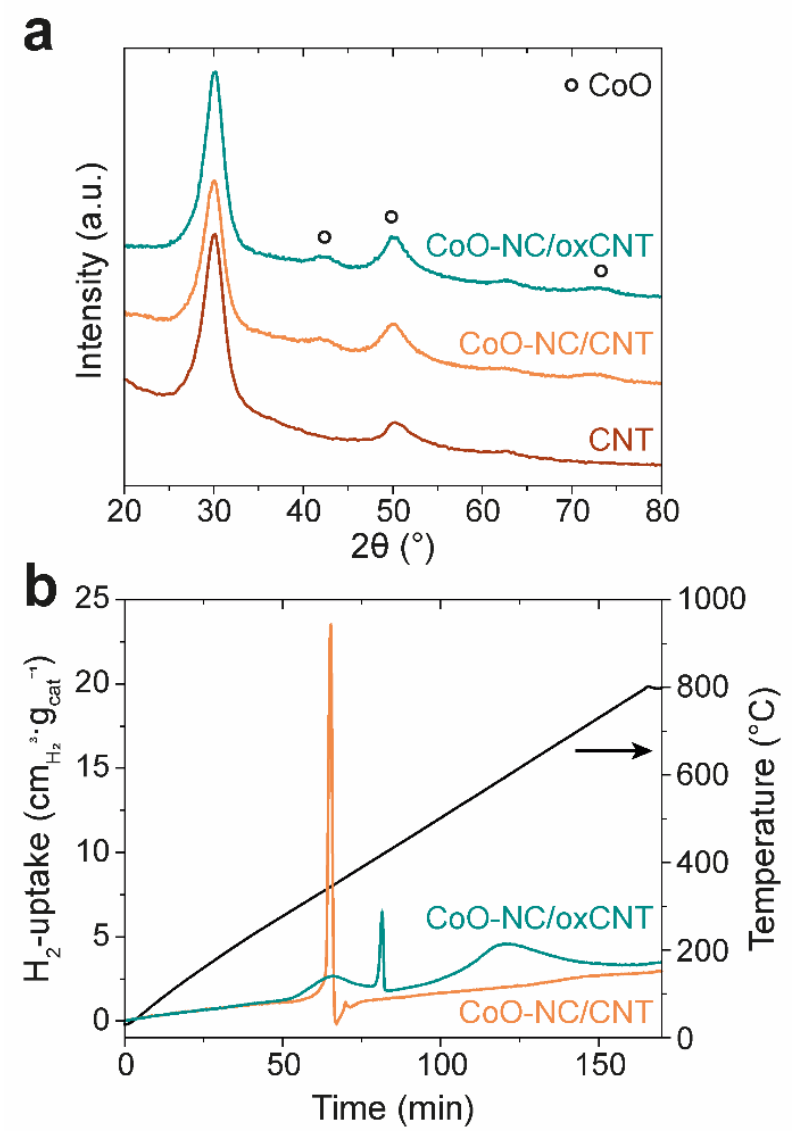

Figure 3.2. Analysis of the crystal structure and reduction behavior of the catalysts. a Ex situ XRD patterns of CNT and the as-prepared samples. $\mathbf{b}$ TPR of the CoO-NC/CNT and CoO-NC/oxCNT in $5 \% \mathrm{H}_{2}$ in Ar with a heating ramp of $5{ }^{\circ} \mathrm{C} \cdot \mathrm{min}^{-1}$.

\subsubsection{Reduction}

Reduction of the pristine samples was investigated using TPR (Figure 3.2b). The CoO-NC were reduced in a single step to $\mathrm{Co}^{\circ}$ (single sharp peak), in contrast to a two-step reduction of $\mathrm{Co}_{3} \mathrm{O}_{4} / \mathrm{CNT}$ that is often reported $\left(\mathrm{CO}_{3} \mathrm{O}_{4}\right.$ to $\mathrm{CoO}$ and $\mathrm{CoO}$ to $\left.\mathrm{Co}^{\circ}\right) \cdot{ }^{6,13}$ Reduction of $\mathrm{CoO}$ occurred at $75{ }^{\circ} \mathrm{C}$ higher temperature with support functionalization. Furthermore, the broad feature around $350{ }^{\circ} \mathrm{C}$ for $\mathrm{CoO}-\mathrm{NC} /$ oxCNT could be related to reduction of oxygencontaining functional groups, defective carbon or cobalt species associated to those, as this contribution was absent in the CNT-supported sample. Finally, the high-intensity peak 
around $600{ }^{\circ} \mathrm{C}$ for $\mathrm{CoO}-\mathrm{NC} / \mathrm{oxCNT}$ was assigned to cobalt-catalyzed methanation of the support, which did not occur for $\mathrm{CoO}-\mathrm{NC} / \mathrm{CNT}$ in the investigated temperature range. The degree of reduction (DOR) of $\mathrm{CoO}$ was determined from the integral of the peak(s) at $350{ }^{\circ} \mathrm{C}$ (CoO-NC/CNT) and 350 and $425{ }^{\circ} \mathrm{C}(\mathrm{CoO}-\mathrm{NC} / \mathrm{oxCNT})$. On CNT, the DOR was $99 \%$, while it was only $46 \%$ on oxCNT. Overall, the different reduction behavior implied stronger interaction of the NC with oxCNT than with pristine CNT.

The catalysts after reduction and passivation were investigated using TEM and STEMHAADF combined with EDX (Figure 3.3). The NC on CNT grew to $11 \mathrm{~nm}$ on average, while those on oxCNT grew only marginally to $8 \mathrm{~nm}$. Thus, functionalization of CNT invoked higher stability of the particles during reduction, probably through more uniform distribution of the NC and/or stronger anchoring of the cobalt particles to the support. ${ }^{13,24}$ On oxCNT, $\sim 3 \mathrm{~nm}$ particles were observed after reduction and passivation, which were absent in the as-prepared state (Appendix B, Figure B2). This meant that re-dispersion of Co had taken place most likely during reduction and/or passivation. The observation of small particles could be linked to the lower DOR derived from TPR (Figure 3.2b), as cobalt oxide particles below $4 \mathrm{~nm}$ are more difficult to reduce. ${ }^{25-27}$ Furthermore, HR-TEM revealed that the cobalt particles were not covered by carbon and that attachment of the NC to the support appeared similar for both catalysts after reduction and passivation (Appendix B, Figure B3).

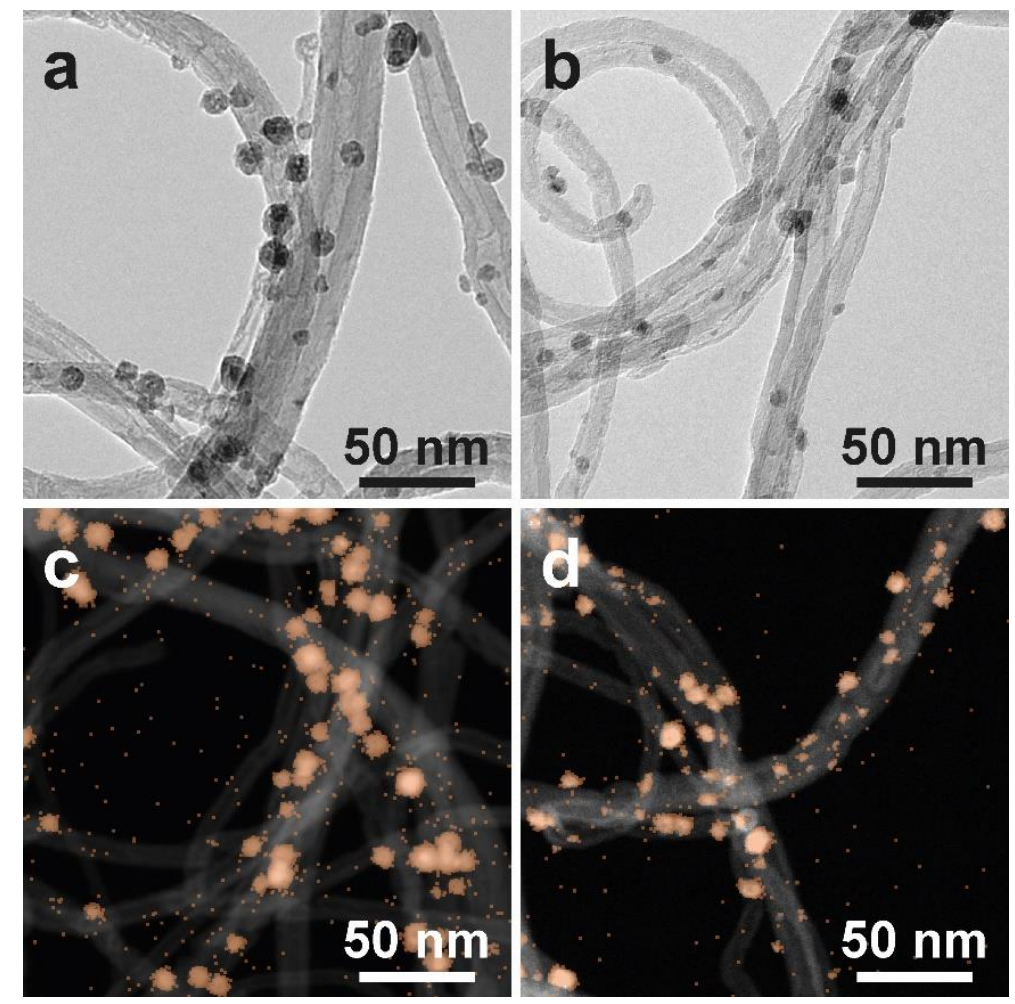

Figure 3.3. Electron microscopy of the catalysts after reduction and passivation. TEM results of a $\mathrm{Co}-\mathrm{NC} / \mathrm{CNT}$ and $\mathbf{b} \mathrm{Co}-\mathrm{NC} / \mathrm{oxCNT}$ are shown together with STEM-HAADF results including cobalt EDX maps of $\mathbf{c} \mathrm{Co}-\mathrm{NC} / \mathrm{CNT}$ and $\mathbf{d} \mathrm{Co}-\mathrm{NC} / \mathrm{oxCNT}$. The cobalt signal is shown in orange. 


\subsubsection{Fischer-Tropsch synthesis}

The cobalt-weight-based activity (cobalt-time yield, CTY) of the catalysts was evaluated over $100 \mathrm{~h}$ on stream (Figure 3.4). After the initial $20 \mathrm{~h}$ on stream, the activity of Co-NC on CNT was substantially higher than on oxCNT. Co-NC/CNT was remarkably active, since CTY values of $26 \cdot 10^{-5} \mathrm{~mol}_{\mathrm{CO}} \cdot \mathrm{g}_{\mathrm{Co}}{ }^{-1} \cdot \mathrm{s}^{-1}$ at the prevailing reaction conditions are rarely reported in literature for nonpromoted cobalt catalysts at the prevailing conditions. Furthermore, the activity profile of $\mathrm{Co}-\mathrm{NC} / \mathrm{CNT}$ was quite stable after the initial period of $20 \mathrm{~h}$ on stream, while the Co-NC/oxCNT deactivated more rapidly.

During the initial $20 \mathrm{~h}$ on stream, both catalysts first activated rapidly before deactivation took over. Activation could be caused by loss of residual ligands under FT conditions through extraction by the formed reaction products. When omitting this strong activation, extrapolation to 0 h gave approximately the same activity for both catalysts and the difference in activity on both supports only set in later. The same was evident from the evolution of $\mathrm{C}_{5_{+}}$-selectivity of the NC-based catalysts with time-on-stream (Appendix B, Figure B4), i.e. initially similar selectivity that later diverged to $82 \%$ on CNT and $78 \%$ on oxCNT, qualitatively in line with previous literature. ${ }^{6}$ These results suggest that differences in catalytic performance take several hours to develop under FT conditions.

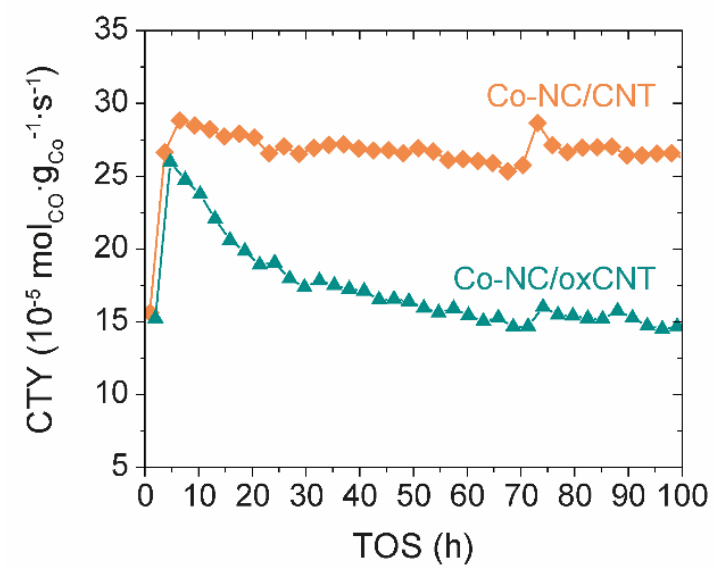

Figure 3.4. Cobalt-time yield of $\mathrm{Co}-\mathrm{NC} / \mathrm{CNT}$ and $\mathrm{Co}-\mathrm{NC} /$ oxCNT during $100 \mathrm{~h}$ on stream at 20 bar, $220{ }^{\circ} \mathrm{C}, \mathrm{H}_{2} / \mathrm{CO}=2$, GHSV $=5950-8900 \mathrm{~h}^{-1}$ and $\mathrm{CO}$ conversion levels between 15 and $40 \%$.

The catalytic results obtained after $\sim 90 \mathrm{~h}$ on stream are summarized in Table 3.3. The cobalt-weight-based activity is reported both as initial and final CTY. Furthermore, surface-volume averaged cobalt particle sizes in the spent and passivated state after $>100 \mathrm{~h}$ on stream were determined by TEM (Table 3.3, Appendix B, Figure B5). Very few particles larger than $20 \mathrm{~nm}$ were observed and omitted in the particle size analysis.

The cobalt particle size distributions of used catalysts were compared to those of the catalysts in the pristine and reduced state to show the evolution of particle size (Figure 3.5a). On pristine CNT, growth of the initially $6 \mathrm{~nm} \mathrm{NC}$ had occurred during reduction and the average size after FT was $10 \mathrm{~nm}$. In contrast, the average NC size on oxCNT remained constant at $\sim 7 \mathrm{~nm}$ during reduction and FT and the particle size distribution was close to its original form (Appendix B, Figure B2). Overall, oxidation of 
CNT prevented average growth of the $\mathrm{Co}-\mathrm{NC}$ during reduction/FT, probably because of a stronger interaction between Co and oxCNT.

Table 3.3. Fischer-Tropsch synthesis results obtained at $20 \mathrm{bar}, 220{ }^{\circ} \mathrm{C}, 2 \mathrm{H}_{2} / \mathrm{CO}(\mathrm{v} / \mathrm{v})$, GHSV $=5950-8900 \mathrm{~h}^{-1}$. The data was averaged between $80-96 \mathrm{~h}$ on stream unless stated otherwise.

\begin{tabular}{|c|c|c|c|c|c|c|c|c|}
\hline Sample & $\begin{array}{l}X_{\mathrm{CO}} \\
(\%)\end{array}$ & $\begin{array}{l}\mathrm{CTY}_{\text {initial }} \\
\text { a,b }\end{array}$ & $\begin{array}{l}\mathrm{CTY}_{\text {final }} \\
\mathrm{a}\end{array}$ & $\begin{array}{l}\mathrm{TOF}_{\text {initial }} \\
\left(10^{-3} \mathrm{~s}^{-1}\right) \\
\mathrm{b,c}\end{array}$ & $\begin{array}{l}\mathrm{TOF}_{\text {final }} \\
\left(10^{-3} \mathrm{~s}^{-1}\right) \\
\mathrm{d}\end{array}$ & $\begin{array}{l}\mathrm{S}_{\mathrm{Cl}} \\
(\% \mathrm{oc})\end{array}$ & $\begin{array}{l}\mathrm{S}_{\mathrm{C} 5+} \\
(\%)\end{array}$ & $\begin{array}{l}\mathrm{D}[3,2] \\
\text { Co, spent } \\
(\mathrm{nm})^{\mathrm{e}}\end{array}$ \\
\hline $\mathrm{Co}-\mathrm{NC} / \mathrm{CNT}$ & 25 & 29 & 26 & 190 & 150 & 11 & 82 & $9.6 \pm 2.9$ \\
\hline Co-NC/oxCNT & 23 & 26 & 15 & 120 & 63 & 12 & 78 & $7.2 \pm 1.6$ \\
\hline
\end{tabular}

${ }^{\mathrm{a}} \operatorname{In}\left(10^{-5} \mathrm{~mol}_{\mathrm{CO}} \cdot \mathrm{g}_{\mathrm{Co}}{ }^{-1} \cdot \mathrm{s}^{-1}\right)$.

${ }^{\mathrm{b}}$ Reported at maximum CTY between $0-10 \mathrm{~h}$ on stream.

c Based on the metallic surface area calculated from the average Co particle size of the reduced and passivated catalyst.

${ }^{\mathrm{d}}$ Based on the metallic surface area calculated from the average Co particle size of the spent and passivated catalyst.

e Determined by TEM; very few large particles (>20 nm) were excluded, if present.

The average particle sizes were used to calculate the cobalt-specific metallic surface areas. From these areas, cobalt-surface-based activities (turnover frequencies, TOF) after $100 \mathrm{~h}$ on stream were derived using the final CTY and particle size of the spent catalyst (Table 3.3). Comparison of the final TOF showed that Co-NC on CNT $\left(150 \cdot 10^{-3} \mathrm{~s}^{-1}\right)$ were significantly more active than on oxCNT $\left(63 \cdot 10^{-3} \mathrm{~s}^{-1}\right)$. In fact, Co-NC/CNT was exceptionally active, considering that TOF values between $10 \cdot 10^{-3} \mathrm{~s}^{-1}$ and $100 \cdot 10^{-3} \mathrm{~s}^{-1}$ are typically obtained. ${ }^{20}$

The TOF was calculated as well for the initial stage of the reaction using the initial (maximal) CTY and particle size of the catalysts after reduction. The TOF decreased from the initial to the final stage of the reaction by $19 \%$ for Co-NC/CNT and by $47 \%$ for Co$\mathrm{NC} / \mathrm{oxCNT}$. Hence, deactivation was predominantly caused by declining intrinsic activity over the course of the reaction and not by particle growth.

As a potential explanation for differences in catalytic performance, we investigated the crystal structure of cobalt in the NC-based catalysts during reduction and under FT conditions using in situ XRD at $10 \mathrm{bar}, 220^{\circ} \mathrm{C}$ and an $\mathrm{H}_{2} / \mathrm{CO}$ ratio of $2 \mathrm{v} / \mathrm{v}$ (Figure $3.5 \mathrm{~b}$, refined patterns in Appendix B, Figure B6). The data was analyzed using Rietveld refinement. To improve the refinement results, it was necessary to include preferred orientations of hcp Co crystallites. Rather than having anisotropic crystallites (for which no evidence was observed in TEM), we propose that this was caused by an intergrown $\mathrm{Co}^{0}$ structure. XRD analysis of metallic cobalt can be complicated by stacking faults and the extent to which these are present depends strongly on the catalyst synthesis and treatments. ${ }^{14,28}$ Our refinement using only hep and fcc Co phases did not account for an intergrowth structure, but did accommodate much of its features by increasing the intensity of the hcp Co (0002) peak, therefore possibly overestimating the fraction of hcp Co. 

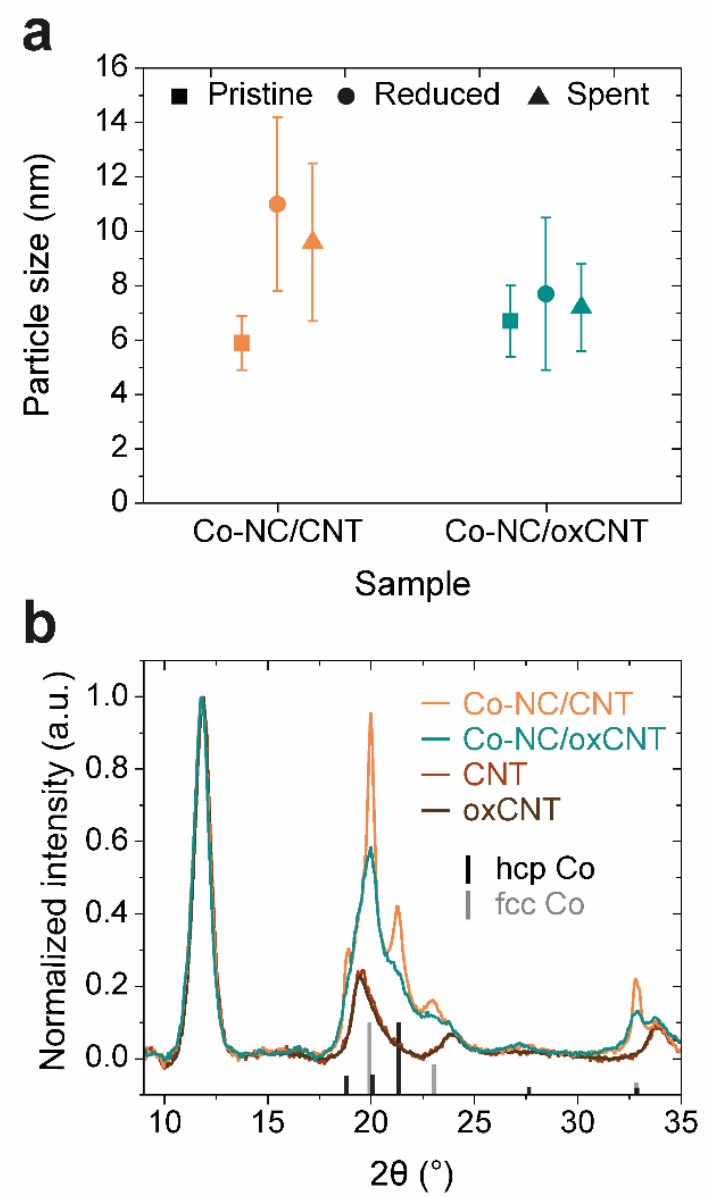

Figure 3.5. Evolution of Co particle size and Co crystal structure under FT conditions. a Average particle sizes $\left(\mathrm{D}[3,2]_{\mathrm{Co}}\right)$ determined by TEM of the catalysts in the as-prepared, reduced and passivated, and spent and passivated state. The error bars indicate the standard deviation. $\mathbf{b}$ Background-subtracted and normalized diffraction patterns of Co-NC on CNT and oxCNT during FT as obtained with in situ XRD and subsequent Rietveld refinement. The diffractograms were obtained between 32 and $42 \mathrm{~h}$ on stream at $10 \mathrm{bar}, 220{ }^{\circ} \mathrm{C}$ and $\mathrm{H}_{2} / \mathrm{CO}=2 \mathrm{v} / \mathrm{v}$ by accumulating five scans of $2 \mathrm{~h}$. No change in the diffraction patterns was apparent over the course of the reaction. Reference patterns of the bare supports were measured at ambient conditions. Contributions from the background and amorphous phases were determined using Rietveld refinement (Appendix B, Figure B7), subtracted from the experimental patterns, and the residual patterns were normalized to the maximum intensity of the CNT (002) peak at $12^{\circ} 2 \theta$. Bars represent reference patterns of hep Co (PDF 04-001-3273) and fcc Co (PDF 04-001-2681).

The width of the peaks between 18 and $25^{\circ} 2 \theta$ was the main difference between the diffraction patterns of $\mathrm{Co}-\mathrm{NC}$ on $\mathrm{CNT}$ and oxCNT and is related to $\mathrm{Co}^{0}$ crystallite size (Figure 3.5b). Especially for Co-NC/CNT, satisfying refinement results were not obtained using a combination of an hcp and fcc phase (Appendix B, Figure B6), probably due to a highly intergrown $\mathrm{Co}^{0}$ structure. The pattern of the $\mathrm{Co}^{0}$ phase on $\mathrm{CNT}$ did bear a strong resemblance to spherical $10 \mathrm{~nm} \mathrm{Co}{ }^{0}$ crystallites reported by Slawińki et al. ${ }^{28}$ with a hcp-fcc stacking probability of 0.5 , i.e. random stacking. In our case, an average crystallite size of $9.9 \mathrm{~nm}$ was also found for the fcc phase, whereas the hcp Co crystallites were only $4.0 \mathrm{~nm}$. 
This small size of hep Co was most likely an artefact caused by inefficient modeling of the intergrowth structure. Therefore, the $\mathrm{Co}^{0}$ crystallites were estimated to be approximately $10 \mathrm{~nm}$ in line with literature ${ }^{28}$ and TEM results, assuming monocrystalline Co-NC. In comparison, the pattern of $\mathrm{Co}-\mathrm{NC} / \mathrm{oxCNT}$ could be modelled better and the average sizes of hcp and fcc Co domains were only 2.9 and $3.9 \mathrm{~nm}$, respectively. As this is smaller than the average particle size from TEM, it meant that polycrystalline Co-NC were formed on oxCNT upon reduction. Furthermore, neither appreciable differences in hcp/fcc ratio nor significant amounts of crystalline $\mathrm{CoO}$ were detected.

More crystalline $\mathrm{Co}^{0}$ appeared present on CNT than on oxCNT, as evidenced by the higher integral between $18-25^{\circ} 2 \theta$. Rietveld refinement indicated a crystalline phase composition of $\mathrm{Co}-\mathrm{NC} / \mathrm{CNT}$ of $8.8 \mathrm{wt} \% \mathrm{Co}^{0}$ and $91.2 \mathrm{wt} \% \mathrm{CNT}$, close to the value from ICP (9.7 $\mathrm{wt} \% \mathrm{Co}$, Table 3.2). In the case of $\mathrm{Co}-\mathrm{NC} /$ oxCNT, the crystalline $\mathrm{Co}^{0}$ phase was only $6.1 \mathrm{wt} \%$ with $93.9 \mathrm{wt} \%$ oxCNT, while its nominal cobalt content from ICP was $9.6 \mathrm{wt} \%$. Oxidation of CNT thus resulted in less crystalline cobalt in the active catalyst, which is qualitatively in line with observations by Chernyak et al. ${ }^{29}$. It is unsure whether the remaining amorphous cobalt was present in ionic or metallic form. Nevertheless, the $32 \%$ lower crystallinity of metallic Co for Co-NC/ oxCNT matched very well with its $36 \%$ lower initial TOF suggesting that differences in crystalline $\mathrm{Co}^{\circ}$ caused the difference in initial TOF.

The diffraction patterns did not change from the final stage of reduction to $40 \mathrm{~h}$ on stream under FT conditions (Appendix B, Figure B7). Hence, restructuring or growth during FT was unlikely, meaning that deactivation occurring on oxCNT (Figure 3.4) had a different origin than changing crystal structure and size. Yu et al. ${ }^{24}$ observed a similar strong deactivation of Co on oxidized CNF. However, they ascribed the deactivation to oxidation of $\mathrm{Co}^{0}$ during FT, which our in situ XRD results did not reveal. In our case, a possible explanation for the deactivation could be enhanced deposition of carbonaceous species $^{30}$ under the influence of defects/oxygen groups on CNT or amorphous cobalt, as has been observed before on oxidized CNT, ${ }^{29}$ but this would require further research. Overall, precise synthesis of $\mathrm{Co}-\mathrm{NC}$ of a particular size combined with uniform attachment to both supports resulted in very similar as-prepared catalysts, varying only in the CNT surface composition. These proved to be excellent model systems to investigate the effect of CNT functionalization on FT in detail.

\subsection{Conclusion}

We investigated the impact of surface oxidation of carbon nanotube (CNT) supports on the structure and performance of cobalt nanocrystals (Co-NC) in the Fischer-Tropsch (FT) synthesis. Well-defined $6 \mathrm{~nm}$ colloidal $\mathrm{CoO}-\mathrm{NC}$ were synthesized and attached to CNT or oxidized CNT (oxCNT) with a loading of $10 \mathrm{wt} \%$ Co. After reduction and passivation, NC growth and complete reduction of the NC were observed on CNT, while growth was absent on oxCNT and only $\sim 50 \% \mathrm{CoO}$ reduction was obtained. The turnover frequency (TOF) of the Co-NC after $100 \mathrm{~h}$ of FT was exceptionally high on CNT with $150 \cdot 10^{-3} \mathrm{~s}^{-1}$ against $63 \cdot 10^{-3}$ $\mathrm{s}^{-1}$ oxCNT. Using in situ XRD, $32 \%$ less crystalline metallic cobalt was found on oxidized CNT under FT conditions, probably causing the $35 \%$ lower initial TOF compared to CoNC/CNT. Furthermore, the Co-NC on oxCNT deactivated more, which could not be 
explained by particle growth, NC restructuring, or oxidation during FT, leaving enhanced deposition of carbonaceous species as a probable cause. This study illustrates the potential of nanocrystal-based model catalyst synthesis for the investigation of structureperformance relationships.

\section{Acknowledgements}

T.W.v.D., B.M.W. and K.P.d.J. acknowledge Shell Global Solutions and the Netherlands Association for Scientific Research (NWO) for funding through the CHIPP framework. H.Y., J.Z. and K.P.d.J. acknowledge the European Research Council, EU FP7 ERC Advanced Grant no. 338846. H.Y. acknowledges the Program for Advancing Strategic International Networks to Accelerate the Circulation of Talented Researchers by JSPS. We thank Arjan den Otter and Nazila Masoud ( $\mathrm{N}_{2}$-physisorption), Helen de Waard (ICP-OES) and Carlos Hernández Mejía (TPR) for the measurements indicated.

\section{References}

1. Prieto, G. et al. Cobalt-Catalyzed Fischer-Tropsch Synthesis: Chemical Nature of the Oxide Support as a Performance Descriptor. ACS Catal. 5, 3323-3335 (2015).

2. Xiong, H., Jewell, L. L. \& Coville, N. J. Shaped Carbons As Supports for the Catalytic Conversion of Syngas to Clean Fuels. ACS Catal. 5, 2640-2658 (2015).

3. Yang, Y. et al. The Correlation of Interfacial Interaction and Catalytic Performance of N-Doped Mesoporous Carbon Supported Cobalt Nanoparticles for Fischer-Tropsch Synthesis. J. Phys. Chem. C118, 268-277 (2014).

4. Bezemer, G. L. et al. Preparation of Fischer-Tropsch cobalt catalysts supported on carbon nanofibers and silica using homogeneous deposition-precipitation. J. Catal. 237, 291-302 (2006).

5. Bezemer, G. L. et al. Cobalt Particle Size Effects in the Fischer-Tropsch Reaction Studied with Carbon Nanofiber Supported Catalysts. J. Am. Chem. Soc. 128, 3956-3964 (2006).

6. Eschemann, T. O. et al. Effect of support surface treatment on the synthesis, structure, and performance of Co/CNT Fischer-Tropsch catalysts. J. Catal. 328, 130-138 (2015).

7. Serp, P., Corrias, M. \& Kalck, P. Carbon nanotubes and nanofibers in catalysis. Appl. Catal. A Gen. 253, 337-358 (2003).

8. Tessonnier, J.-P. et al. Analysis of the structure and chemical properties of some commercial carbon nanostructures. Carbon 47, 1779-1798 (2009).

9. Ros, T. G., Van Dillen, A. J., Geus, J. W. \& Koningsberger, D. C. Surface Oxidation of Carbon Nanofibres. Chem. - Eur. J. 8, 1151-1162 (2002).

10. Bitter, J. H. Nanostructured carbons in catalysis a Janus material-industrial applicability and fundamental insights. J. Mater. Chem. 20, 7312-7321 (2010).

11. Zhang, $\mathrm{H}$. et al. The nature of cobalt species in carbon nanotubes and their catalytic performance in Fischer-Tropsch reaction. J. Mater. Chem. 19, 9241-9249 (2009).

12. Vosoughi, V., Badoga, S., Dalai, A. K. \& Abatzoglou, N. Effect of Pretreatment on Physicochemical Properties and Performance of Multiwalled Carbon Nanotube Supported Cobalt Catalyst for Fischer-Tropsch Synthesis. Ind. Eng. Chem. Res. 55, 6049-6059 (2016). 
13. Chernyak, S. A. et al. Co catalysts supported on oxidized CNTs: evolution of structure during preparation, reduction and catalytic test in Fischer-Tropsch synthesis. Appl. Catal. A Gen. 523, 221-229 (2016).

14. Ducreux, O., Rebours, B., Lynch, J., Roy-Auberger, M. \& Bazin, D. Microstructure of Supported Cobalt Fischer-Tropsch Catalysts. Oil Gas Sci. Technol. - Rev. d'IFP Energies Nouv. 64, 49-62 (2009).

15. Sadeqzadeh, M. et al. Identification of the active species in the working alumina-supported cobalt catalyst under various conditions of Fischer-Tropsch synthesis. Catal. Today 164, 62-67 (2011).

16. Gnanamani, M. K., Jacobs, G., Shafer, W. D. \& Davis, B. H. Fischer-Tropsch synthesis: Activity of metallic phases of cobalt supported on silica. Catal. Today 215, 13-17 (2013).

17. Liu, J.-X., Su, H.-Y., Sun, D.-P., Zhang, B.-Y. \& Li, W.-X. Crystallographic Dependence of CO Activation on Cobalt Catalysts: HCP versus FCC. J. Am. Chem. Soc. 135, 16284-16287 (2013).

18. Lyu, S. et al. Role of Active Phase in Fischer-Tropsch Synthesis: Experimental Evidence of CO Activation over Single-Phase Cobalt Catalysts. ACS Catal. 8, 7787-7798 (2018).

19. Zaera, F. Nanostructured materials for applications in heterogeneous catalysis. Chem. Soc. Rev. 42, 2746-2762 (2013).

20. van Deelen, T. W., Su, H., Sommerdijk, N. A. J. M. \& de Jong, K. P. Assembly and activation of supported cobalt nanocrystal catalysts for the Fischer-Tropsch synthesis. Chem. Commun. 54, 2530-2533 (2018).

21. Iablokov, V. et al. Size-Controlled Model Co Nanoparticle Catalysts for $\mathrm{CO}_{2}$ Hydrogenation: Synthesis, Characterization, and Catalytic Reactions. Nano Lett. 12, 3091-3096 (2012).

22. Cats, K. H. \& Weckhuysen, B. M. Combined Operando X-ray Diffraction/Raman Spectroscopy of Catalytic Solids in the Laboratory: The $\mathrm{Co} / \mathrm{TiO}_{2}$ Fischer-Tropsch Synthesis Catalyst Showcase. ChemCatChem 8, 1531-1542 (2016).

23. Xie, J. et al. Promoted cobalt metal catalysts suitable for the production of lower olefins from natural gas. Nat. Commun. 10, 167 (2019).

24. Yu, Z., Borg, Ø., Chen, D., Rytter, E. \& Holmen, A. Role of surface oxygen in the preparation and deactivation of carbon nanofiber supported cobalt Fischer-Tropsch catalysts. Top. Catal. 45, 69-74 (2007).

25. Wolf, M., Kotzé, H., Fischer, N. \& Claeys, M. Size dependent stability of cobalt nanoparticles in high conversion Fischer-Tropsch environment. Faraday Discuss. 197, 243-268 (2017).

26. van Steen, E. et al. Stability of Nanocrystals: Thermodynamic Analysis of Oxidation and Rereduction of Cobalt in Water/Hydrogen Mixtures. J. Phys. Chem. B109, 3575-3577 (2005).

27. Tsakoumis, N. E. et al. Evaluation of Reoxidation Thresholds for $\gamma-\mathrm{Al}_{2} \mathrm{O}_{3}$-Supported Cobalt Catalysts under Fischer-Tropsch Synthesis Conditions. J. Am. Chem. Soc. 139, 3706-3715 (2017).

28. Stawiński, W. A., Zacharaki, E., Fjellvåg, H. \& Sjåstad, A. O. Structural Arrangement in ClosePacked Cobalt Polytypes. Cryst. Growth Des. 18, 2316-2325 (2018).

29. Chernyak, S. A. et al. Effect of Co crystallinity on $\mathrm{Co} / \mathrm{CNT}$ catalytic activity in $\mathrm{CO} / \mathrm{CO}_{2}$ hydrogenation and CO disproportionation. Appl. Surf. Sci. 372, 100-107 (2016).

30. Peña, D. et al. Molecular structure and localization of carbon species in alumina supported cobalt Fischer-Tropsch catalysts in a slurry reactor. Catal. Today 228, 65-76 (2014). 
Cobalt nanocrystals on carbon nanotubes in the Fischer-Tropsch synthesis: impact of support oxidation 


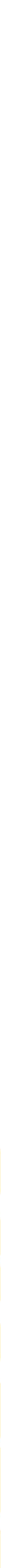




\title{
Chapter 4
}

\section{Preparation of cobalt nanocrystals supported on metal oxides to study particle growth in Fischer-Tropsch catalysts}

\begin{abstract}
Colloidal synthesis of nanocrystals (NC) followed by their attachment to a support and activation is a promising route to prepare model catalysts for research on structureperformance relationships. Here, we investigated the suitability of this method to prepare well-defined $\mathrm{Co} / \mathrm{TiO}_{2}$ and $\mathrm{Co} / \mathrm{SiO}_{2}$ catalysts for the Fischer-Tropsch (FT) synthesis with high control over the cobalt particle size. To this end, Co-NC of 3, 6, 9 and $12 \mathrm{~nm}$ with narrow size distributions were synthesized and attached uniformly on either $\mathrm{TiO}_{2}$ or $\mathrm{SiO}_{2}$ supports with comparable morphology and Co loadings of 2-10 wt \%. After activation in

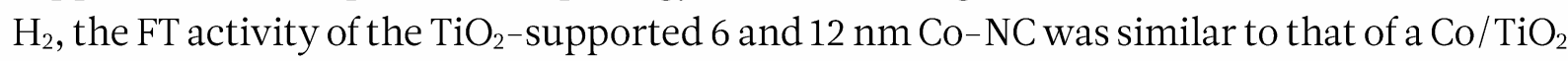
catalyst prepared by impregnation, showing that full activation was achieved and relevant catalysts had been obtained, however, $3 \mathrm{~nm} \mathrm{Co}-\mathrm{NC}$ on $\mathrm{TiO}_{2}$ were less active than anticipated. Analysis after FT revealed that all Co- $\mathrm{NC}$ on $\mathrm{TiO}_{2}$ as well as $3 \mathrm{~nm} \mathrm{Co-} \mathrm{NC}$ on $\mathrm{SiO}_{2}$

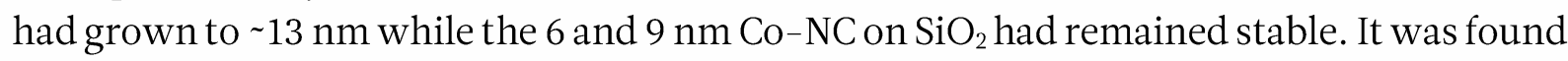
that the $3 \mathrm{~nm} \mathrm{Co}-\mathrm{NC}$ on $\mathrm{TiO}_{2}$ already grew to $10 \mathrm{~nm}$ during activation in $\mathrm{H}_{2}$. Furthermore, substantial amounts of Co (up to $60 \%$ ) migrated from the Co-NC to the support during activation on $\mathrm{TiO}_{2}$ against only $15 \%$ on $\mathrm{SiO}_{2}$. We showed that the stronger interaction between cobalt and $\mathrm{TiO}_{2}$ leads to enhanced catalyst restructuring as compared to $\mathrm{SiO}_{2}$. These findings demonstrate the potential of the NC-based method to produce relevant model catalysts to investigate phenomena that could not be studied using conventionally synthesized catalysts.
\end{abstract}

Based on: van Deelen, T. W., Nijhuis, J. J., Krans, N. A., Zečević, J. \& de Jong, K. P. ACS Catal. 8, 10581-10589 (2018). 


\subsection{Introduction}

Colloidal synthesis of cobalt nanocrystals (NC) offers the possibility to develop new model catalysts that can potentially aid in linking the structure of cobalt catalysts to their performance in the Fischer-Tropsch (FT) synthesis reaction. ${ }^{1-3}$ However, base metal NC are challenging to utilize in supported catalysts. First, homogeneous attachment of base metal NC on a support can be hindered by magnetic interactions between the NC. ${ }^{4}$ Second, removal of the stabilizing ligand shell can be incomplete, blocking active sites and lowering catalytic activity, or harsh treatments are applied that may compromise the well-defined nature of the catalyst. ${ }^{5,6}$ We demonstrated uniform attachment and successful ligand removal for $\mathrm{Co}-\mathrm{NC}$ supported on carbon nanotubes by applying a mild, low-temperature oxidation to the NC in suspension prior to attachment. ${ }^{7}$ The cobalt-catalyzed FT reaction is reported to be structure sensitive, related to both cobalt particle size ${ }^{8,9}$ and cobalt crystal structure $^{10-12}$. So far, most research involving Co-NC for FT concentrated either on slurryphase FT, in which the unsupported $\mathrm{Co}-\mathrm{NC}$ remain in suspension ${ }^{13-15}$, or on $\mathrm{Co}-\mathrm{NC}$ supported on irreducible oxides (mainly $\mathrm{SiO}_{2}$ or $\left.\mathrm{Al}_{2} \mathrm{O}_{3}\right)^{16-23}$. However, reducible oxides such as $\mathrm{TiO}_{2}$ are also interesting supports for cobalt-based FT catalysts, for both industry ${ }^{24}$ and academia ${ }^{25}$.

$\mathrm{TiO}_{2}$-supported cobalt catalysts can be considered more complex than $\mathrm{Co} / \mathrm{SiO}_{2}$ or $\mathrm{Co} / \mathrm{Al}_{2} \mathrm{O}_{3}$, since $\mathrm{TiO}_{2}$ can be partially reduced under reaction conditions, leading to special interphase phenomena known as strong metal-support interactions (SMSI) ${ }^{26,27}$ The effects of these metal- $\mathrm{TiO}_{2}$ interactions remain poorly understood, in part because synthesis procedures for well-defined catalysts are lacking, especially for $<10 \mathrm{~nm}$ Co particles. Monodisperse particles on $\mathrm{TiO}_{2}$ can be synthesized with conventional methods, but the control over the average particle size is limited. ${ }^{28,29}$ Furthermore, there are only few reports of $\mathrm{NC}$-based $\mathrm{Co} / \mathrm{TiO}_{2}$ catalysts and these either contained boron impurities owing to the synthesis method ${ }^{30,31}$ or the $\mathrm{Co}-\mathrm{NC}$ were $10 \mathrm{~nm}$ or larger ${ }^{18,32}$. Therefore, it would be highly desirable to expand the applicability of $\mathrm{Co}-\mathrm{NC}$ to $\mathrm{TiO}_{2}$ as support.

In this chapter, we report the synthesis and catalytic performance of well-defined catalysts containing 3-12 nm Co-NC on $\mathrm{TiO}_{2}$ and $\mathrm{SiO}_{2}$ and demonstrate their use as model systems by studying particle growth under FT conditions. The Co-NC were synthesized by a hot-injection method. After low-temperature oxidation, the CoO-NC were homogeneously attached to either $\mathrm{TiO}_{2}$ or $\mathrm{SiO}_{2}$. Their catalytic performance was comparable to that of $\mathrm{Co} / \mathrm{TiO}_{2}$ prepared by impregnation, except for $3 \mathrm{~nm} \mathrm{Co-} \mathrm{NC}$ on $\mathrm{TiO}_{2}$, whose activity was substantially lower. $\mathrm{The} \mathrm{TiO}_{2}$-supported Co particles and $3 \mathrm{~nm} \mathrm{Co}-\mathrm{NC}$ on $\mathrm{SiO}_{2}$ sintered during reduction and/or FT, indicating that both the support and initial NC size affected the stability of Co-NC under reaction conditions. We show that the followed NC-based methodology enabled the synthesis of size-controlled $\mathrm{Co} / \mathrm{TiO}_{2}$ and $\mathrm{Co} / \mathrm{SiO}_{2}$ with relevant catalytic performance. Their potential as model systems is underlined by investigating NC particle growth, revealing phenomena that are not observed with conventionally prepared catalysts. 
Preparation of cobalt nanocrystals supported on metal oxides to study particle growth in Fischer-Tropsch catalysts

\subsection{Experimental}

\subsubsection{Synthesis of CoO nanocrystals}

Cobalt nanocrystals of different sizes were synthesized by a hot injection method based on literature ${ }^{33}$ and our previous work ${ }^{7}$. A schematic summary of the NC synthesis procedure is shown in Appendix C, Figure C1. The benefit of this method is that the required chemicals contain no elements such as phosphorus or boron that might affect the catalyst and its final catalytic performance. The syntheses were performed on a Schlenk line in $\mathrm{N}_{2}$ atmosphere. In a typical synthesis, $65 \mathrm{mg}$ oleic acid (Sigma-Aldrich, $90 \%$, technical grade) was degassed under vacuum at $100{ }^{\circ} \mathrm{C}$ for $30 \mathrm{~min}$ in a 3-necked $100 \mathrm{~mL}$ flask with a cooler and two septa. After flushing and switching to $\mathrm{N}_{2}, 7.5 \mathrm{~mL} 1,2$-dichlorobenzene was added under inert atmosphere and the solution was heated to temperatures between $165-182^{\circ} \mathrm{C}$ related to the desired NC size. Simultaneously, inside a glovebox, $270 \mathrm{mg}$ dicobalt octacarbonyl (Acros Organics, $95 \%$, stabilized) was dissolved in $1.5 \mathrm{~mL} \mathrm{1,2-dichlorobenze.} \mathrm{This} \mathrm{cobalt}$ precursor was then rapidly injected (needle: $\varnothing 0.9 \times 70 \mathrm{~mm}$ ) into the heated solution under $750 \mathrm{rpm}$ magnetic stirring and decomposed instantly. The reaction was quenched after 20 min using a water bath. Once at ambient temperature, the $\mathrm{N}_{2}$ supply was switched off and one septum was removed to allow low-temperature oxidation by air-exposure under $650 \mathrm{rpm}$ stirring. After $1 \mathrm{~h}$, the mixture was divided over two glass centrifuge tubes and filled to $20 \mathrm{~mL}$ with 2-propanol (Sigma-Aldrich, >99\%, LC-MS Chromasolv) and centrifuged at $2200 \mathrm{G}$ for $30 \mathrm{~min}$. The supernatant was decanted and the residue redispersed in $0.5 \mathrm{~mL} \mathrm{n}$-hexane (Acros Organics, 99+\%) per tube by sonication. The tubes were filled to $20 \mathrm{~mL}$ with 2-propanol and centrifuged at $2200 \mathrm{G}$ for $40 \mathrm{~min}$. This washing cycle was repeated twice and after the last step, the residue was re-dispersed and combined in a total of $2 \mathrm{~mL}$-hexane.

\subsubsection{Attachment of $\mathrm{CoO}$ nanocrystals to a support}

The Co-NC were attached to a support according the method developed by Casavola et al. ${ }^{34}$. A schematic summary of the $\mathrm{NC}$ attachment procedure is shown in Appendix C, Figure C1. The supports were $\mathrm{TiO}_{2}$ (Evonik, Aeroxide P25) or $\mathrm{SiO}_{2}$ (Evonik, Aerosil OX 50), both sieved to a fraction of $75-150 \mu \mathrm{m}$. To regulate the final cobalt loading, between $0.4-2.5 \mathrm{~g}$ of support was suspended in a mixture of the colloidal suspension and 5-31 mL 1-octadecene (Sigma-Aldrich, $90 \%$, technical grade) inside a $100 \mathrm{~mL} 3$-necked flask. A cooler, glass plug and septum were connected to the flask, the magnetic stirring was set to $400 \mathrm{rpm}$ and the suspension was degassed under vacuum at $100{ }^{\circ} \mathrm{C}$ for $15 \mathrm{~min}$, during which the $\mathrm{n}$-hexane from the colloidal suspension evaporated. The setup was then flushed, switched to $\mathrm{N}_{2}$ and heated to $200{ }^{\circ} \mathrm{C}$. The heating was stopped after $30 \mathrm{~min}$ at $200{ }^{\circ} \mathrm{C}$ and the solution cooled to ambient temperature in approximately $30 \mathrm{~min}$. The suspension was divided over two glass centrifuge tubes, centrifuged at $1500 \mathrm{G}$ for $5 \mathrm{~min}$ and the supernatant was decanted. The residue was re-suspended in $2 \mathrm{~mL}$-hexane, sonicated for $1 \mathrm{~min}$, diluted with $6 \mathrm{~mL}$ acetone, centrifuged at $1500 \mathrm{G}$ for $5 \mathrm{~min}$ and the supernatant was again decanted. This washing cycle was repeated five times, followed by re-suspending the residue in $20 \mathrm{~mL}$ acetone, sonicating for $1 \mathrm{~min}$, centrifuging at $1500 \mathrm{G}$ for $5 \mathrm{~min}$ and decanting the supernatant. Afterwards, the samples were dried at $60{ }^{\circ} \mathrm{C}$ for $1 \mathrm{~h}$ in static air, followed by 
$120{ }^{\circ} \mathrm{C}$ for $3 \mathrm{~h}$ in static air and $80^{\circ} \mathrm{C}$ for $3 \mathrm{~h}$ under vacuum. Finally, the samples were sieved to a grain size of $75-150 \mu \mathrm{m}$.

\subsubsection{Incipient wetness impregnation}

A comparison $\mathrm{Co} / \mathrm{TiO}_{2}$ sample was prepared by incipient wetness impregnation following the procedure by Eschemann et al. ${ }^{28}$. First, $2 \mathrm{~g} \mathrm{TiO}_{2}$ (Evonik, Aeroxide P25) was sieved to 75-150 $\mu \mathrm{m}$ and then dried at $80^{\circ} \mathrm{C}$ for $1 \mathrm{~h}$ under vacuum. The vacuum was released and impregnated directly afterwards with a pore-filling amount of precursor solution consisting of $\mathrm{Co}\left(\mathrm{NO}_{3}\right)_{2} \cdot 6 \mathrm{H}_{2} \mathrm{O}$ (Acros Organics, 99+\%) in Mili-Q water. The solution was added dropwise under magnetic and manual stirring. After the addition, the powder was dried and heat treated in a U-shaped, glass reactor in fluidized bed mode $\left(1 \mathrm{~L} \cdot \mathrm{min}^{-1}\right.$ upward flow of $\mathrm{N}_{2}$ ). Drying took place at $80^{\circ} \mathrm{C}$ for $2 \mathrm{~h}$ and was followed directly by a thermal treatment at $250{ }^{\circ} \mathrm{C}$ for $2 \mathrm{~h}$, both with $2{ }^{\circ} \mathrm{C} \cdot \mathrm{min}^{-1}$. The sample was finally sieved again to a grain size of $75-150 \mu \mathrm{m}$.

\subsubsection{Plasma cleaning}

Optionally, pristine Co-NC samples were plasma cleaned to remove the ligands prior to reduction and FT. Typically, $100 \mathrm{mg}$ of sample was treated for $8 \mathrm{~h}$ in a home-made container inserted into a Fischione Model 1020 Plasma Cleaner. The plasma was generated using a $25 \% \mathrm{O}_{2}$ in Ar flow and a 13.56 MHz oscillating electromagnetic field.

\subsubsection{Characterization}

Transmission electron microscopy (TEM) was performed either on a Tecnai 12 (FEI), operated at $120 \mathrm{kV}$, or on a Tecnai 20 (FEI), operated at $200 \mathrm{kV}$. TEM samples of Co-NC suspensions were prepared by sonicating and dropcasting a diluted sample directly on a carbon-coated TEM grid. To prepare a sample of supported Co-NC, the solid was first suspended in 2-propanol by sonication and then dropcasted on a TEM grid. In the case of samples after catalysis, the samples were washed three times using toluene and $30 \mathrm{~min}$ sonication and once using $n$-hexane to remove the hydrocarbon reaction products before dropcasting on a TEM grid. The sizes of $>200$ particles were determined manually using ImageJ software. All particle sizes are reported as the Sauter mean diameter (volume/surface mean, $\mathrm{D}[3,2]$ ) of the equivalent metallic cobalt particles, meaning that (partial) cobalt oxide particles were corrected based on the densities of the oxide and the metal.

Scanning transmission electron microscopy combined with energy-dispersive X-ray spectroscopy (STEM-EDX) was performed in high-angle annular dark-field (HAADF) mode on a Talos F200X (FEI), equipped with a high-brightness field emission gun (X-FEG) and a Super-X G2 EDX detector and operated at $200 \mathrm{kV}$. Particle size analysis was similar to that of TEM, with the exception that $<200$ particles were analyzed for some samples, depending on the particle size and cobalt loading.

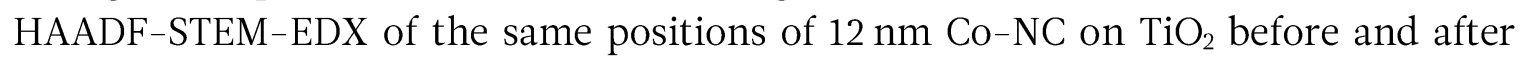
reduction was performed using a SiN TEM chip (TEMWindow SN100-A50Q33). After imaging the pristine sample, the the SiN TEM chip was transferred to a glass U-shaped reactor and the sample on the chip was reduced ex situ inside in $25 \% \mathrm{H}_{2}$ in Ar flow at $350{ }^{\circ} \mathrm{C}$ 
Preparation of cobalt nanocrystals supported on metal oxides to study particle growth in Fischer-Tropsch catalysts

for $8 \mathrm{~h}$ with $1^{\circ} \mathrm{C} \cdot \mathrm{min}^{-1}$ and cooled down afterwards under the same atmosphere. Subsequently, the chip was transferred back to the microscope and analyzed again. During the transfer, the sample was exposed to air at ambient conditions.

$\mathrm{N}_{2}$-physisorption of the bare supports was measured at $-196{ }^{\circ} \mathrm{C}$ on a Tristar 3000 (Micromeritics). The samples were dried in He at $300{ }^{\circ} \mathrm{C}$ for $14-20 \mathrm{~h}$ prior to the analysis.

Inductively coupled plasma-optical emission spectroscopy (ICP-OES) was carried out on a SPECTRO ARCOS and the sample was digested in aqua regia to extract the cobalt.

Temperature programed reduction (TPR) profiles were measured on an Autochem 2920 (Micromeritics). Approximately $100 \mathrm{mg}$ of sample was reduced in $5 \% \mathrm{H}_{2}$ in Ar flow from $50{ }^{\circ} \mathrm{C}$ to $800{ }^{\circ} \mathrm{C}$ with $5{ }^{\circ} \mathrm{C} \cdot \mathrm{min}^{-1}$.

$\mathrm{X}$-ray diffraction (XRD) was performed on a Bruker D2 Phaser, equipped with a Co radiation source $(\lambda=1.789 \AA)$. The sample was measured between $20-100^{\circ} 2 \theta$ with an increment of $0.05^{\circ}$.

\subsubsection{Fischer-Tropsch synthesis}

The catalytic performance in the Fischer-Tropsch synthesis was investigated using an Avantium Flowrence 16 parallel reactor setup. Stainless steel plug flow reactors of 2.0 or $2.6 \mathrm{~mm}$ ID were loaded with 25-110 mg catalyst (75-150 $\mu \mathrm{m}$ ) diluted with $100 \mathrm{mg} \mathrm{SiC} \mathrm{(200-}$ $425 \mu \mathrm{m})$. Prior to reaction, the catalysts were reduced in situ at 1 bar in $25 \% \mathrm{H}_{2}$ in $\mathrm{He}$ flow at either $350{ }^{\circ} \mathrm{C}\left(\mathrm{TiO}_{2}\right.$-samples) or $500{ }^{\circ} \mathrm{C}\left(\mathrm{SiO}_{2}\right.$-samples $)$ for $8 \mathrm{~h}$ with $1{ }^{\circ} \mathrm{C} \cdot \mathrm{min}^{-1}$. Afterwards, the reactors were cooled to $180{ }^{\circ} \mathrm{C}$ and pressurized to $20 \mathrm{bar}$. At these conditions, the atmosphere was switched to synthesis gas with $2 \mathrm{H}_{2} / \mathrm{CO}(\mathrm{v} / \mathrm{v})$ and $5 \% \mathrm{He}$ as internal standard. Subsequently, the reactors were heated to $220{ }^{\circ} \mathrm{C}$ with $1{ }^{\circ} \mathrm{C} \cdot \mathrm{min}^{-1}$ and product analysis was started after $1 \mathrm{~h}$. The reaction products were analyzed online using an Agilent 7890A GC equipped with two channels. The first channel contained an Agilent J\&W PoraBOND Q column connected to an FID to detect the hydrocarbon products and the second one consisted of an ShinCarbon-ST column connected to a TCD to analyze the permanent gasses.

The catalytic activity was reported as CO conversion $\left(\mathrm{X}_{\mathrm{CO}}\right)$, cobalt time yield $(\mathrm{CTY})$ and turnover frequency (TOF). The TOF was based on the activity and the Sauter mean diameter $(\mathrm{D}[3,2])$ of the spent $\mathrm{Co}-\mathrm{NC}$ after correction for a $3 \mathrm{~nm} \mathrm{CoO}$ passivation layer, assuming a cross-sectional area of $0.0662 \mathrm{~nm}^{2}$ per cobalt atom. ${ }^{35}$ The selectivity $(\% \mathrm{c})$ to $\mathrm{C}_{1}-$ $\mathrm{C}_{4}$ products was defined as $\mathrm{S}_{\mathrm{C} 1-\mathrm{C} 4}=100 \cdot \mathrm{F}_{\mathrm{Cn}} \cdot \mathrm{n} \cdot\left(\mathrm{F}_{\mathrm{CO}, \mathrm{in}} \cdot \mathrm{X}_{\mathrm{CO}}\right)^{-1}$ with $\mathrm{n}$ being the product's carbon number and $\mathrm{F}$ being the flow of the corresponding hydrocarbon or $\mathrm{CO}$. The selectivity to $\mathrm{C}_{5+}$ was defined as $\mathrm{S}_{\mathrm{C} 5+}=100-\mathrm{S}_{\mathrm{Cl}-4}$.

\subsection{Results and discussion}

\subsubsection{Cobalt nanocrystal synthesis}

Well-defined cobalt nanocrystals were synthesized with distinct sizes between 3-12 nm and a polydispersity of $11-18 \%$ (Figure 4.1, additional particle size distributions in Appendix C, Figure C2). The Co-NC were designated as CoX, with X denoting the average diameter of the NC. The size of the $\mathrm{Co}-\mathrm{NC}$ size was regulated by varying the temperature of 
the hot injection synthesis, which is in line with literature. ${ }^{33,36}$ The Co-NC size correlated with the synthesis temperature in the range of $170-183{ }^{\circ} \mathrm{C}$ and became less reproducible at lower temperature (Figure 4.1d). After the synthesis, low-temperature oxidation by airexposure at ambient conditions was applied to facilitate attachment and activation of the Co-NC. ${ }^{7}$ Of the synthesized Co-NC in Figure 4.1d, those of 3, 6, 9 and $12 \mathrm{~nm}$ were attached to $\mathrm{TiO} 2$ or $\mathrm{SiO} 2$, thus omitting the $\mathrm{Co}-\mathrm{NC}$ of 4 and $8 \mathrm{~nm}$.

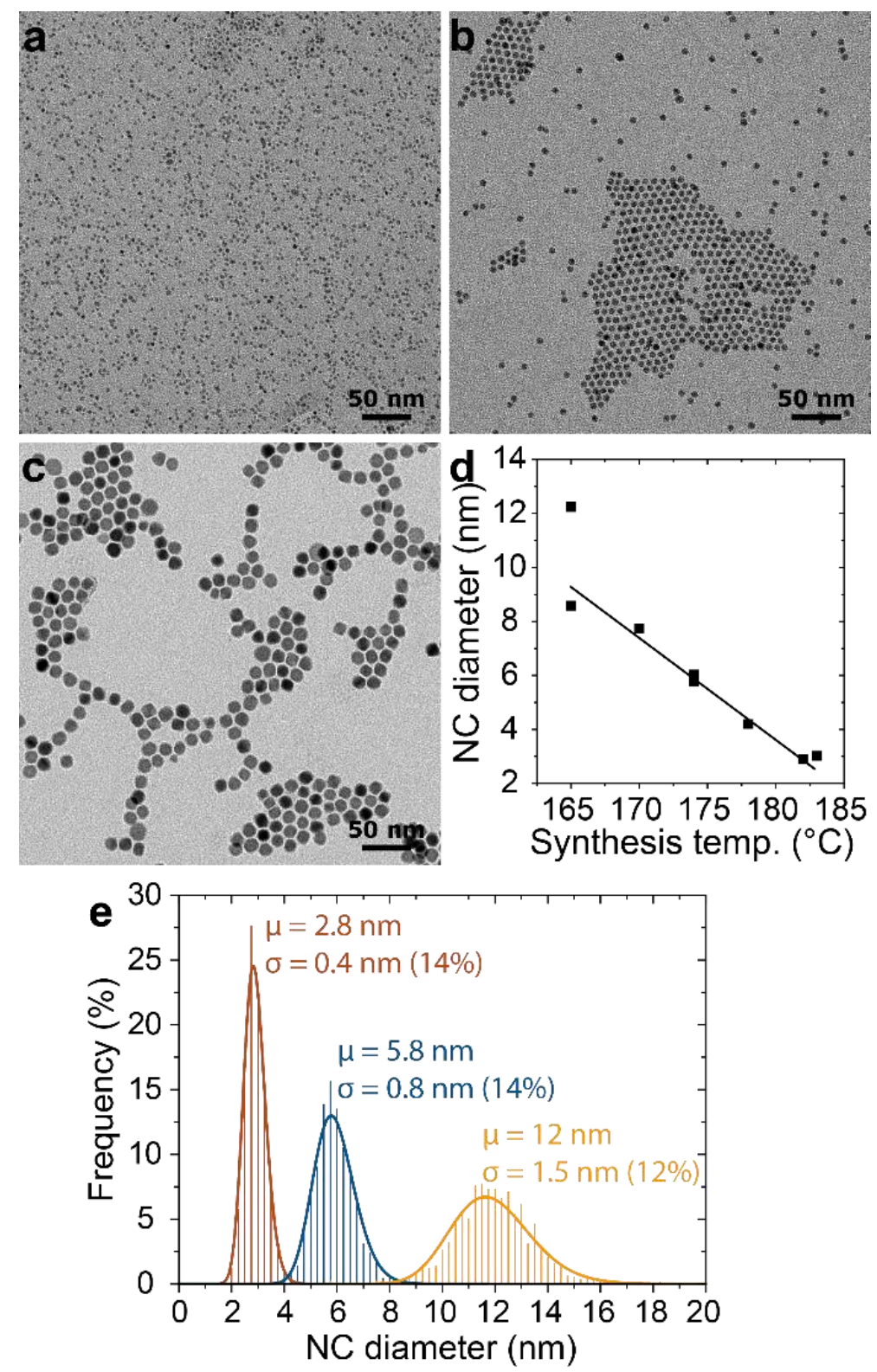

Figure 4.1. Overview of the as-synthesized Co-NC. TEM images of $\mathbf{a}$ Co3, $\mathbf{b}$ Co6 and $\mathbf{c} \mathrm{Co} 12$ and $\mathbf{d}$ the resulting $\mathrm{Co}-\mathrm{NC}$ size as a function of the hot-injection temperature. e Histograms and lognormal distributions of selected sizes of Co-NC.

\subsubsection{Catalyst preparation}

The Co-NC were attached to titania and to silica with comparable specific surface areas $\left(\sim 50 \mathrm{~m}^{2} \cdot \mathrm{g}^{-1}\right)$ but different mesopore volumes $\left(0.3\right.$ and $0.1 \mathrm{~mL} \cdot \mathrm{g}^{-1}$, respectively) as determined by $\mathrm{N}_{2}$-physisorption (Appendix C, Figure C3, Table $\mathrm{C1}$ ). The cobalt loading was 
adjusted to obtain similar cobalt metal surface areas per unit weight of catalyst in the pristine samples with the aim to avoid major differences in catalyst-weight-based activities during FT (Table 4.1). Furthermore, the Co-NC on the supports were analyzed with TEM (Figure 4.2). The Co-NC distribution was uniform over both supports, similar to previous results with a carbon support. ${ }^{7,34}$ In addition, a reference $\mathrm{Co} / \mathrm{TiO}_{2}$ sample was prepared by conventional techniques, in this case incipient wetness impregnation of a cobalt nitrate solution (designated as IWI-Co/ $\mathrm{TiO}_{2}$, details in Table 4.1 and TEM results in Appendix C, Figure C4). Its average particle size was most comparable to $\mathrm{Co6} / \mathrm{TiO}_{2}$, but the particle size distribution was substantially broader.

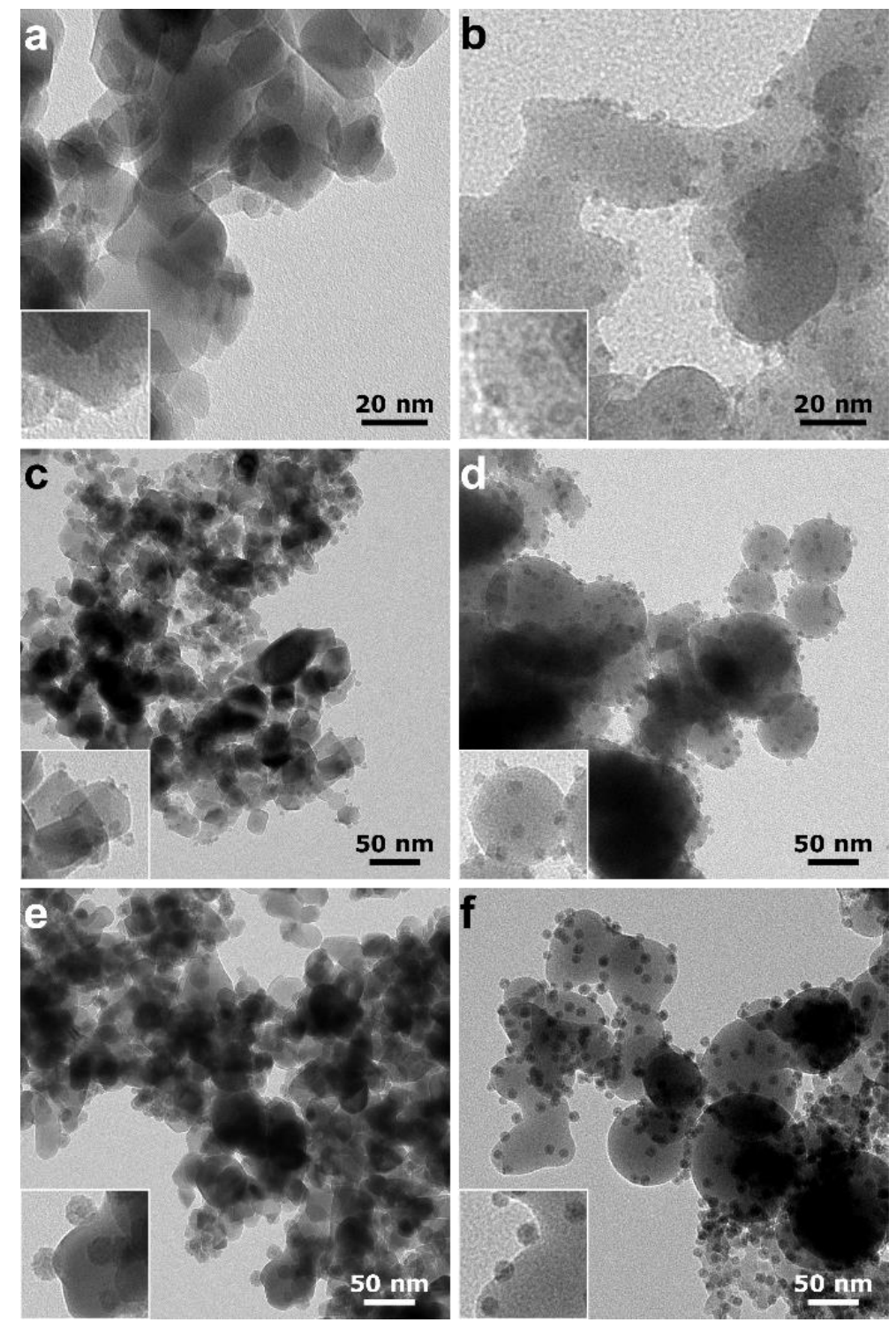

Figure 4.2. Overview of the $\mathrm{Co}-\mathrm{NC}$ attached to a support. The $\mathrm{TiO}_{2}$-supported samples are in the left column and the $\mathrm{SiO}_{2}$-supported ones in the right column. a Co3 $/ \mathrm{TiO}_{2}, \mathbf{b ~ C o} 3 / \mathrm{SiO}_{2}, \mathbf{c ~ C o} / \mathrm{TiO}_{2}, \mathbf{d}$ $\mathrm{Co6} / \mathrm{SiO}_{2}$, e $\mathrm{Co} 12 / \mathrm{TiO}_{2}$ and $\mathrm{f} \mathrm{Co} / \mathrm{SiO}_{2}$. The insets are at double the magnification of the original image. 
Table 4.1. Characteristics of the supported Co-NC and IWI-Co samples. The Sauter mean diameter ( \pm standard deviation) of the Co-NC was analyzed by TEM before attachment and recalculated to the corresponding CoO particle sizes. The Co loading was determined by ICP-OES. Based on these two results, a Co specific surface area of the pristine catalysts was calculated.

\begin{tabular}{llll}
\hline Sample & $\begin{array}{l}\mathrm{D}[3,2]_{\mathrm{Co}-\mathrm{NC}} \\
(\mathrm{nm})\end{array}$ & Co loading $(\mathbf{w t} \%)$ & $\begin{array}{l}\text { Initial Co surface } \\
\text { area }\left(\mathrm{m}_{\mathrm{Co}}{ }^{2} \cdot \mathrm{g}_{\mathrm{Cat}}{ }^{-1}\right)\end{array}$ \\
\hline $\mathrm{Co} 3 / \mathrm{TiO}_{2}$ & $2.9 \pm 0.4$ & 2.4 & 5.7 \\
$\mathrm{Co6} / \mathrm{TiO}_{2}$ & $6.0 \pm 0.8$ & 4.6 & 5.5 \\
$\mathrm{Co} 12 / \mathrm{TiO}_{2}$ & $12 \pm 1.5$ & 8.7 & 5.4 \\
$\mathrm{IWI}-\mathrm{Co} / \mathrm{TiO}_{2}$ & $7.3 \pm 2.5$ & 6.7 & 6.2 \\
$\mathrm{Co} 3 / \mathrm{SiO}_{2}$ & $2.8 \pm 0.5$ & 3.6 & 6.8 \\
$\mathrm{Co6} / \mathrm{SiO}_{2}$ & $5.6 \pm 0.6$ & 6.8 & 9.2 \\
$\mathrm{Co9} / \mathrm{SiO}_{2}$ & $8.6 \pm 1.1$ & 9.6 & 7.7 \\
\hline
\end{tabular}

The sizes of the Co-NC remained unchanged during the attachment treatment as concluded from TEM (Figure 4.2). The Co- $\mathrm{NC}$ of 3 and $6 \mathrm{~nm}$ consisted completely of $\mathrm{CoO}^{7}$ while for the 9 and $12 \mathrm{~nm} \mathrm{Co}-\mathrm{NC}$ only an outer layer of $\mathrm{CoO}$ was formed and a metallic core with higher contrast was observed. This $\mathrm{CoO}$ shell had a thickness of $4.5 \mathrm{~nm}$ in the case of $\mathrm{Co12} / \mathrm{TiO}_{2}$, which exceeds the $\mathrm{CoO}$ passivation layer of $3 \mathrm{~nm}$ that is typically observed upon exposure of metallic cobalt particles to air at ambient conditions.$^{37}$ The increased oxidation of the Co-NCs could have occurred during drying at $120^{\circ} \mathrm{C}$, as this is close to the temperature range were Kirkendall effects have been observed. ${ }^{38}$ The Kirkendall effect occurs upon oxidation of metals at elevated temperatures and leads to the formation of hollow metal oxide particles. ${ }^{6,39}$

The reducibility of the Co- $\mathrm{NC}$ on $\mathrm{TiO}_{2}$ samples was investigated by temperatureprogrammed reduction (TPR, Figure 4.3a). Two reduction peaks were observed between $250-450{ }^{\circ} \mathrm{C}$ for all samples next to a negative peak at higher temperatures, which is attributed to the desorption/decomposition of remaining ligands. ${ }^{7}$ As expected, the reduction temperature shifts to lower values with increasing particle size, because small oxide particles often display lower reducibility. ${ }^{40-42}$ Furthermore, the total $\mathrm{H}_{2}$-uptake related to the first two peaks increased with the particle size following the increase in cobalt content. Interestingly, when comparing the total $\mathrm{H}_{2}$-uptake of the $\mathrm{Co}-\mathrm{NC} / \mathrm{TiO}_{2}$ samples normalized to the amount of $\mathrm{Co}$, the $\mathrm{H}_{2}$-uptake of $\mathrm{Co}_{2} / \mathrm{TiO}_{2}$ was only $89-92 \%$ of that of the 3 and $6 \mathrm{~nm} \mathrm{NC} \mathrm{(deconvolutions} \mathrm{in} \mathrm{Appendix} \mathrm{C,} \mathrm{Figure} \mathrm{C5).} \mathrm{This} \mathrm{indicated} \mathrm{that}$ $\sim 10 \%$ of the cobalt in $\mathrm{Co1} / 2 / \mathrm{TiO}_{2}$ was still present in the core of the NC as metallic cobalt, which was in line with the earlier TEM observation of a metallic core and a CoO passivation layer (Figure 4.2). 


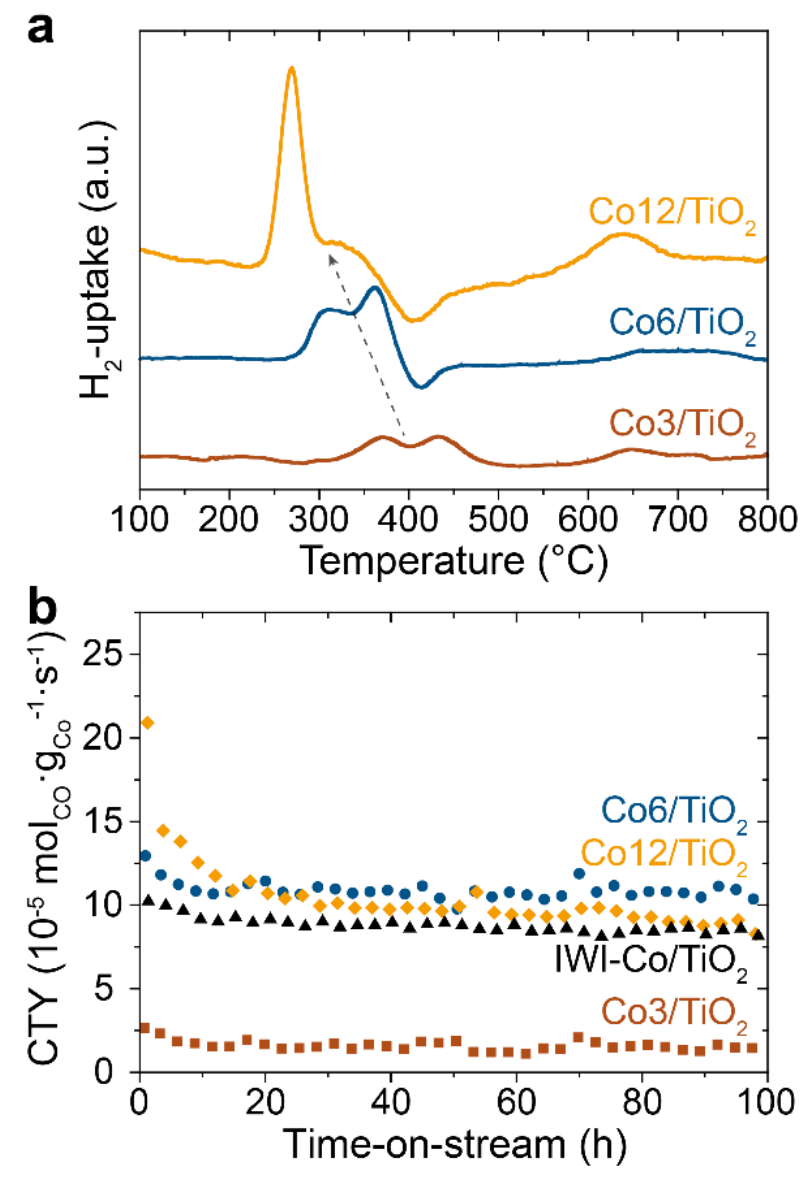

Figure 4.3. Reduction and catalytic activity of the $\mathrm{TiO}_{2}$-supported samples. a Reduction profiles obtained by TPR normalized to the mass of sample and $\mathbf{b}$ catalytic activity (CTY) for FT as a function of time-on-stream performed at $20 \mathrm{bar}, 220^{\circ} \mathrm{C}, 2 \mathrm{H}_{2} / \mathrm{CO}(\mathrm{v} / \mathrm{v})$, GHSV $=1950-5900 \mathrm{~h}^{-1}$ and $\mathrm{X}_{\mathrm{CO}}=5^{-}$ $60 \%$

\subsubsection{Fischer-Tropsch synthesis}

The cobalt-weight-based activity (cobalt time yield, CTY) of the $\mathrm{TiO}_{2}$-supported samples was evaluated over $100 \mathrm{~h}$ on stream (Figure 4.3b, Table 4.2). $\mathrm{Co6} / \mathrm{TiO}_{2}$ was the most active after $100 \mathrm{~h}$ followed by $\mathrm{Co} 12 / \mathrm{TiO}_{2}$, which was initially more active but deactivated faster. $\mathrm{Co} 3 / \mathrm{TiO}_{2}$ was a factor 5 less active per unit weight of cobalt over the entire time range studied. Interestingly, the 6 and $12 \mathrm{~nm}$ Co-NC catalysts were similar or even higher in CTY than the IWI-Co/ $\mathrm{TiO}_{2}$ catalyst, indicating that these $\mathrm{Co}-\mathrm{NC}$ were adequately activated and that relevant catalytic results were obtained with these NC-based model systems. Furthermore, the selectivity towards $\mathrm{C}_{5+}$ products was around $85 \%$ for all $\mathrm{TiO}_{2}$-supported samples, except for $\mathrm{Co} 3 / \mathrm{TiO}_{2}$ whose $\mathrm{C}_{5+}$-selectivity was only $57 \%$ (Table 4.2 ).

The catalytic activity of the $\mathrm{Co} / \mathrm{SiO}_{2}$ samples was investigated over $100 \mathrm{~h}$ on stream at CO conversion levels between 15-30\% (Appendix C, Figure C6, Table 4.2). The cobaltweight-based activities of $\mathrm{Co} 6 / \mathrm{SiO}_{2}$ and $\mathrm{Co} 9 / \mathrm{SiO}_{2}$ were the highest of all investigated catalysts. The $3 \mathrm{~nm}$ Co-NC displayed again the lowest activity of the series, yet $\mathrm{Co} 3 / \mathrm{SiO}_{2}$ was a factor 6 more active per unit weight of cobalt than $\mathrm{Co} 3 / \mathrm{TiO}_{2}$. The $\mathrm{C}_{5_{+}}-$selectivities of the $\mathrm{SiO}_{2}$-supported catalysts varied between 76 and $79 \%$ and were overall lower than that 
of most $\mathrm{TiO}_{2}$-supported catalysts $(\sim 85 \%)$. This observation corresponds well with earlier findings of $\mathrm{TiO}_{2}$ promoting the $\mathrm{C}_{5+}$-selectivity of cobalt catalysts in FT. ${ }^{43}$

The deactivation and observed differences in activity could be caused by several mechanisms, such as carbon deposition ${ }^{44}$, metal-support compound formation ${ }^{45}$ or net particle growth ${ }^{45}$. To investigate the occurrence of particle growth in more detail, the spent catalysts were analyzed with TEM (Table 4.2, Fig 4, Appendix C, Figure C7). All Co-NC supported on $\mathrm{TiO}_{2}$, as well as the IWI-Co/ $\mathrm{TiO}_{2}$, had grown to $\sim 13 \mathrm{~nm}$ on average, which was in line with recently reported particle sizes of a $\mathrm{Co}-\mathrm{Re} / \mathrm{TiO}_{2}$ catalyst after various periods on stream. ${ }^{45} \mathrm{On} \mathrm{SiO}_{2}$, the initially $3 \mathrm{~nm} \mathrm{Co}-\mathrm{NC}$ had also sintered to $13 \mathrm{~nm}$, while the 6 and $9 \mathrm{~nm}$ Co-NC were stable.

Using the particle sizes of the spent catalysts and the activities at 90-100 h on stream, the surface-specific activities (turnover frequencies, TOF) were calculated (Table 4.2). The TOF of all $\mathrm{TiO}_{2}$-supported catalysts except $\mathrm{Co} 3 / \mathrm{TiO}_{2}$ was around $65 \cdot 10^{-3} \mathrm{~s}^{-1}$, in line with previous reports. ${ }^{46-48}$ The identical TOF of 6 and $12 \mathrm{~nm} \mathrm{Co-NC}$ on $\mathrm{TiO}_{2}$ and the IWI-Co/ $\mathrm{TiO}_{2}$ catalyst showed that the active cobalt sites were not affected by the presence of residual ligands or other factors specific to the NC-based synthesis method. The apparent TOF of $\mathrm{Co} 3 / \mathrm{TiO}_{2}$, based on the particle size of the catalyst and the end-of-run activity, was only $12 \cdot 10^{-3} \mathrm{~s}^{-1}$ (Table 4.2). This was remarkable, because the Co particles in $\mathrm{Co} 3 / \mathrm{TiO}_{2}$ had grown outside of the size regime where particle size effects typically cause low activities $(<6-$ $8 \mathrm{~nm}) .^{8,9,16}$ Therefore, a TOF similar to the others $\left(65 \cdot 10^{-3} \mathrm{~s}^{-1}\right)$ should have been obtained. Instead, the low apparent TOF was ascribed to loss of cobalt from the metal particles to the $\mathrm{TiO}_{2}$ resulting in less metallic surface area available for catalysis. Judging from the decrease in TOF, up to $80 \%$ of the cobalt was lost in this manner.

Table 4.2. Catalytic performance at $20 \mathrm{bar}, 220^{\circ} \mathrm{C}$ and $2 \mathrm{H}_{2} / \mathrm{CO}(\mathrm{v} / \mathrm{v})$. The reported data was obtained after $90-100 \mathrm{~h}$ on stream. The TOF was based on the end-of-run activity and Co particle size $(\mathrm{D}[3,2])$ of the spent samples. The Sauter mean diameter ( ${ }$ standard deviation) of the Co-NC was analyzed in the spent and passivated state and corrected for the lattice expansion due to the $3 \mathrm{~nm}$ CoO passivation layer.

\begin{tabular}{lllllllll}
\hline Sample & $\begin{array}{l}\mathrm{GHSV} \\
\left(\mathbf{h}^{-1}\right)\end{array}$ & $\begin{array}{l}\mathrm{X}_{\mathrm{CO}} \\
(\%)\end{array}$ & $\begin{array}{l}\mathrm{CTY} \\
\mathbf{a}\end{array}$ & $\begin{array}{l}\mathrm{TOF} \\
\left(\mathbf{1 0}^{-3} \mathbf{s}^{-1}\right)\end{array}$ & $\begin{array}{l}\mathrm{S}_{\mathrm{C} 1} \\
(\%)\end{array}$ & $\begin{array}{l}\mathrm{S}_{\mathrm{C} 2 \mathrm{C} 4} \\
(\%)\end{array}$ & $\begin{array}{l}\mathrm{S}_{\mathrm{C} 5+} \\
(\%)\end{array}$ & $\begin{array}{l}\mathrm{D}[3,2]_{\text {spent }} \\
(\mathbf{n m})\end{array}$ \\
\hline $\mathrm{Co} 3 / \mathrm{TiO}_{2}$ & 1950 & 4.6 & 1.4 & 12 & 21 & 21 & 57 & $15 \pm 5.1$ \\
$\mathrm{Co6} / \mathrm{TiO}_{2}$ & 3100 & 23 & 11 & 66 & 9.2 & 8.1 & 83 & $11 \pm 3.3$ \\
$\mathrm{Co} 12 / \mathrm{TiO}_{2}$ & 6900 & 24 & 8.6 & 68 & 8.2 & 5.9 & 86 & $13 \pm 4.0$ \\
$\mathrm{IWI}-\mathrm{Co} / \mathrm{TiO}_{2}$ & 7550 & 18 & 8.4 & 68 & 8.7 & 7.0 & 84 & $14 \pm 5.9$ \\
$\mathrm{Co} 3 / \mathrm{SiO}_{2}$ & 1500 & 25 & 8.5 & 67 & 13 & 12 & 76 & $13 \pm 5.5$ \\
$\mathrm{Co6} / \mathrm{SiO}_{2}$ & 5500 & 16 & 21 & 82 & 14 & 11 & 76 & $6.7 \pm 1.4$ \\
$\mathrm{Co9} / \mathrm{SiO}_{2}$ & 10750 & 21 & 19 & 106 & 13 & 9 & 79 & $9.6 \pm 2.1$ \\
\hline
\end{tabular}

${ }^{\mathrm{a}}$ in $10^{-5} \mathrm{~mol}_{\mathrm{CO}} \cdot \mathrm{g}_{\mathrm{Co}}{ }^{-1} \cdot \mathrm{s}^{-1}$

The TOF of the $\mathrm{SiO}_{2}$-supported catalysts increased with initial particle size (Table 4.2). In the case of the initially $3 \mathrm{~nm} \mathrm{Co-NC}$ on $\mathrm{SiO}_{2}$, the TOF was $67 \cdot 10^{-3} \mathrm{~s}^{-1}$, which was similar to most $\mathrm{Co} / \mathrm{TiO}_{2}$ catalysts and already somewhat higher than previously reported for $\mathrm{Co} / \mathrm{SiO}_{2}$ catalysts. ${ }^{49}$ However, the intrinsic activities of 6 and $9 \mathrm{~nm} \mathrm{Co-NC}$ on $\mathrm{SiO}_{2}$ were 
Preparation of cobalt nanocrystals supported on metal oxides to study particle growth in Fischer-Tropsch catalysts

exceptionally high in comparison to literature. A possible explanation for the different TOF of $\mathrm{Co}-\mathrm{NC}$ and conventional catalysts could be that the Co-NC approach led to cobalt particles with a higher fraction of hcp Co, which is more active in FT than fcc $\mathrm{Co}^{10-12,50}$. Alternatively, the Co-NC could have less interaction with the support, because the Co-NC were formed prior to attachment to the support. For example, when comparing the TEM results of spent $\mathrm{Co} / \mathrm{SiO}_{2}$, the grown particles of $\mathrm{Co} 3 / \mathrm{SiO}_{2}$ were mainly confined between primary $\mathrm{SiO}_{2}$ particles where the contact area with the support was maximal (Appendix C, Figure C7). The $\mathrm{Co}-\mathrm{NC}$ in $\mathrm{Co} 6 / \mathrm{SiO}_{2}$ and $\mathrm{Co} 9 / \mathrm{SiO}_{2}$, on the other hand, were still distributed uniformly over the support and as such, a higher fraction of the cobalt surface area would be accessible to reactants.

Overall, the employed NC-based approach to catalyst synthesis yielded well-defined model systems with reasonable control over the initial NC size and relevant catalytic performance. It is therefore anticipated that these model catalysts are suitable to investigate structure-performance relationships and already some interesting phenomena were observed here, especially on the growth of $\mathrm{NC}$ of specific sizes under reaction conditions. These observations will be investigated and discussed in more detail in the next section.

\subsubsection{Sintering of cobalt nanocrystals}

To investigate the growth of $\mathrm{Co}-\mathrm{NC}$, the particle sizes were analyzed using TEM after reduction as well as after catalysis (Figure 4.4). $3 \mathrm{~nm} \mathrm{Co-NC}$ on $\mathrm{TiO}_{2}$ sintered to particles of $10 \mathrm{~nm}$ already during reduction and the particles grew further to $15 \mathrm{~nm}$ during FT. The $3 \mathrm{~nm}$ Co-NC on $\mathrm{SiO}_{2}$ ultimately grew to the same extent, but in this case the particles predominantly sintered during FT. The reduction barely affected the particle size of $\mathrm{Co} 3 / \mathrm{SiO}_{2}$ even though the reduction was performed at $500{ }^{\circ} \mathrm{C}$.

EDX maps of the reduced and passivated $\mathrm{TiO}_{2}$-supported catalysts and $\mathrm{Co} 3 / \mathrm{SiO}_{2}$ were quantified to investigate the location of Co on the supports (Table 4.3, see Appendix C, Figure C8 for results and experimental details). Compared to ICP (Table 4.1), the total cobalt loading from EDX was 30-50 \% higher on $\mathrm{TiO}_{2}$ and $50 \%$ lower on $\mathrm{SiO}_{2}$. Therefore, relative differences for one and the same sample were considered more representative than the absolute values.

For the reduced and passivated $\mathrm{Co} / \mathrm{TiO}_{2}$ samples, $\sim 2.5 \mathrm{wt} \%$ of $\mathrm{Co}$ was found dispersed in the support and not in particles, regardless of $\mathrm{NC}$ size and loading. In the case of $\mathrm{Co} 3 / \mathrm{TiO}_{2}$, this amounted to approximately $60 \%$ of the total cobalt content from EDX and might be partially responsible for its low CTY. Furthermore, the Co signal in the EDX maps occasionally followed the contours of $\mathrm{TiO}_{2}$ particles with increased intensity at the surface (Appendix C, Figure C8). This means that a higher Co concentration existed at the surface than in the bulk of the $\mathrm{TiO}_{2}$ particles. In contrast, only $16 \%$ of the Co was found on the silica support for $\mathrm{Co} 3 / \mathrm{SiO}_{2}$ and the remaining $84 \%$ was still present in Co particles. Consequently, no contours of the support were observed in the Co signal from EDX at locations without any particles (Appendix C, Figure C8). These EDX results show that, during reduction, cobalt migrated to the support more with $\mathrm{TiO}_{2}$ than with $\mathrm{SiO}_{2}$, as illustrated in Figure 4.4c,d. These cobalt species on the support were not detected in XRD (Appendix C, Figure C9), probably because of their low concentration and/or low 
crystallinity. Additionally, the amount of cobalt on the support was assumed to remain constant during FT, because the $\mathrm{Co} / \mathrm{TiO}_{2}$ catalysts did not activate during FT, which would be expected if the cobalt species on the support were reduced under reaction conditions (Figure 4.3b).
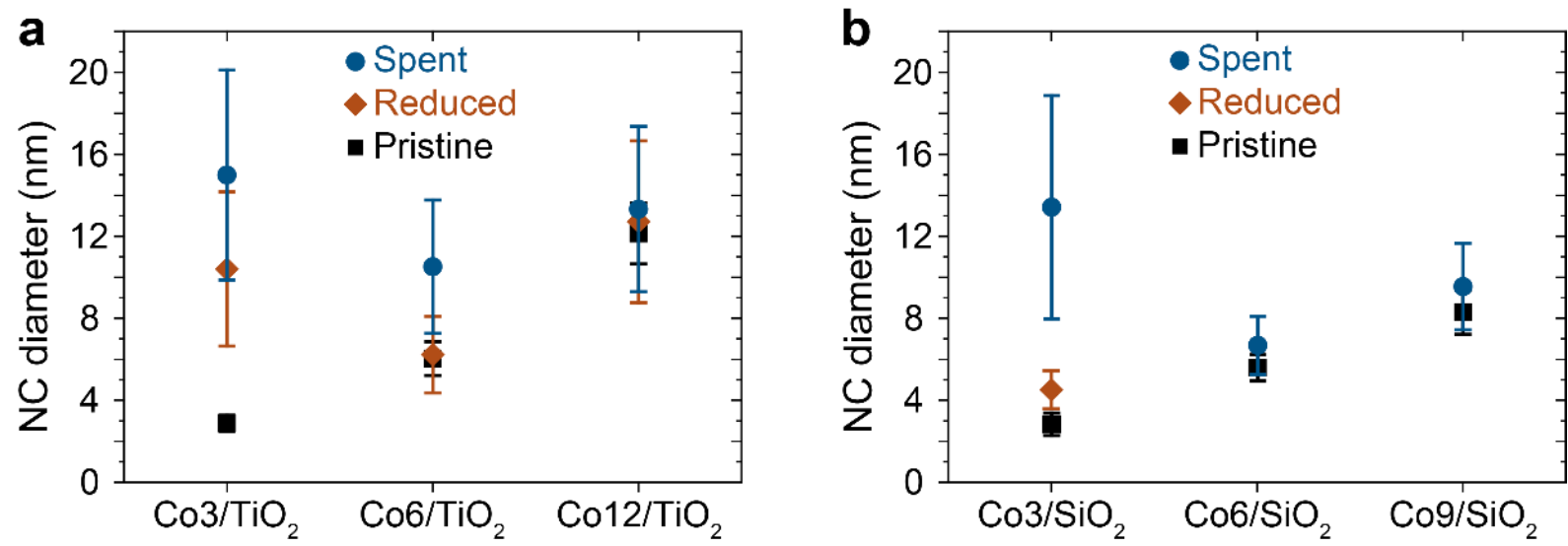

C<smiles>[TeH]</smiles>

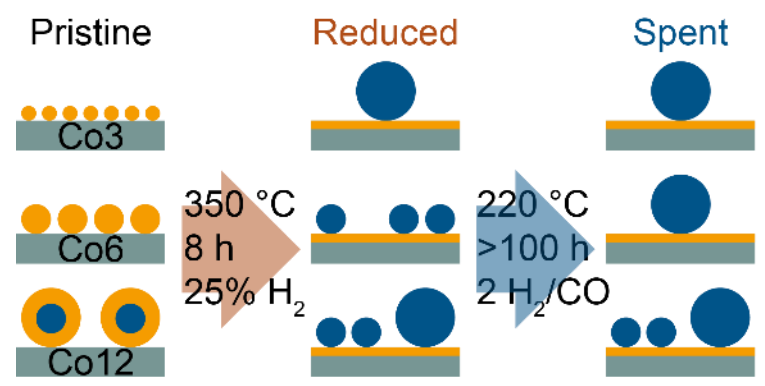

d<smiles>[SeH4]</smiles>

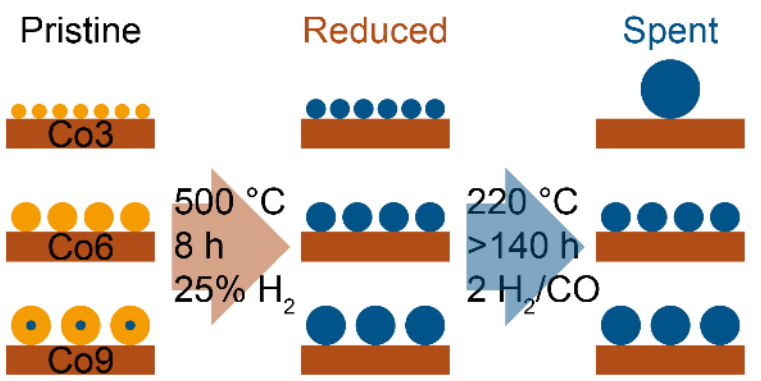

Figure 4.4. Average cobalt particle sizes of the samples in the pristine, reduced and spent state. a,b Average Co-NC sizes from TEM analysis of the $\mathrm{TiO}_{2}-$ and $\mathrm{SiO}_{2}$-supported samples, respectively. The error bars give the standard deviation of the average particle size. Schematic illustration of the sintering behavior of the different sizes on $\mathbf{c} \mathrm{TiO}_{2}$ and on $\mathbf{d ~ \mathrm { SiO } _ { 2 }}$ after different treatments. In these illustrations, $\mathrm{Co}^{2+}$ or $\mathrm{Co}^{3+}$ is depicted in yellow and $\mathrm{Co}^{0}$ in blue. Migration of $\mathrm{Co}$ to $\mathrm{TiO}_{2}$ during reduction was observed by EDX.

Table 4.3. Quantification of the cobalt present in the support and in particles after reduction of $\mathrm{Co} / \mathrm{TiO}_{2}$ and $\mathrm{Co} 3 / \mathrm{SiO}_{2}$. The total Co loading and the fractions of $\mathrm{Co}$ in particles and $\mathrm{Co}$ in the support were derived from HAADF-STEM-EDX (Appendix C, Figure C8).

\begin{tabular}{llllll}
\hline Sample & $\begin{array}{l}\mathrm{Co}_{\text {total }} \\
\mathbf{( w t} \%)\end{array}$ & $\begin{array}{l}\mathrm{Co}_{\text {particles }} \\
\mathbf{( w t} \%)\end{array}$ & $\begin{array}{l}\mathrm{Co}_{\text {support }} \\
\mathbf{( w t} \%)\end{array}$ & $\begin{array}{l}\mathrm{Co}_{\text {particles }} / \\
\mathrm{Co}_{\text {total }}\end{array}$ & $\begin{array}{l}\mathrm{Co}_{\text {support }} / \\
\text { Co }_{\text {total }}\end{array}$ \\
\hline $\mathrm{Co} 3 / \mathrm{TiO}_{2}$ & 3.3 & 1.3 & 2.0 & 0.38 & 0.62 \\
$\mathrm{Co} 6 / \mathrm{TiO}_{2}$ & 6.8 & 4.1 & 2.7 & 0.61 & 0.39 \\
$\mathrm{Co} 12 / \mathrm{TiO}_{2}$ & 11 & 9.2 & 2.2 & 0.81 & 0.19 \\
$\mathrm{Co} 3 / \mathrm{SiO}_{2}$ & 2.4 & 2.0 & 0.4 & 0.84 & 0.16 \\
\hline
\end{tabular}


Preparation of cobalt nanocrystals supported on metal oxides to study particle growth in Fischer-Tropsch catalysts

Small particles have a higher thermodynamic potential ${ }^{51}$ and are therefore more prone to sintering. ${ }^{52,53}$ Interestingly, in these earlier studies, growth took place via monomers that were created upon exposure to $\mathrm{CO}$ under reaction conditions. For example, in the case of $\mathrm{Co} / \mathrm{Al}_{2} \mathrm{O}_{3}$, cobalt subcarbonyls were proposed as monomers that migrate over the support and give rise to particle growth via Ostwald ripening. ${ }^{54-56}$ However, in our $\mathrm{Co} 3 / \mathrm{TiO}_{2}$ sample, a large part of the growth took place already during reduction, so sintering was not induced by $\mathrm{CO}$. When combining these results with the tendency of $60 \%$ of the cobalt in the NC to 'leach' to the surface of the $\mathrm{TiO}_{2}$, we propose that different mobile species, possibly cobalt titanates ${ }^{57}$, spread out over the support and cause a few particles to grow large. This hypothesis is in line with thermodynamic calculations (Appendix C, Figure C10), which showed that it is thermodynamically possible to form small amounts of cobalt titanates under reduction conditions, and agrees with previous observations. ${ }^{58-60}$ Notably, Cats et al. ${ }^{58}$ observed a thin layer of Co species surrounding $\mathrm{TiO}_{2}$ particles, albeit in spent and not in reduced catalysts.

On $\mathrm{SiO}_{2}$, the formation of cobalt silicates during reduction is thermodynamically limited (Appendix C, Figure C10) and the growth of $3 \mathrm{~nm} \mathrm{Co-NC} \mathrm{occurred} \mathrm{mainly} \mathrm{during}$ FT. The sintering might therefore be ascribed to $\mathrm{CO}$-induced Ostwald ripening as observed before by Kistamurthy et al. ${ }^{61}$ or particle migration and coalescence. Furthermore, $8 \mathrm{~h}$ plasma cleaning of the pristine samples to remove ligands reduced the particle growth for $\mathrm{Co} 3 / \mathrm{SiO}_{2}$, but not for the $\mathrm{Co} / \mathrm{TiO}_{2}$ (Appendix C, Figure C11). This further confirmed that the mechanism responsible for particle growth is different on both supports even though the final particle size is similar.

$6 \mathrm{~nm} \mathrm{Co}-\mathrm{NC}$ on $\mathrm{TiO}_{2}$ did not grow during reduction (Figure 4.4). The driving force for sintering during reduction was thus less than for $\mathrm{Co} 3 / \mathrm{TiO}_{2}$ because of the larger particle size. However, the $\mathrm{NC}$ on $\mathrm{TiO}_{2}$ did grow to $11 \mathrm{~nm}$ during FT. Growth of the $6 \mathrm{~nm} \mathrm{Co}-\mathrm{NC}$ was not observed on $\mathrm{SiO}_{2}$ even after catalysis, showing that the Co- $\mathrm{NC}$ are less stable on $\mathrm{TiO}_{2}$ compared to $\mathrm{SiO}_{2}$. Interestingly, $\mathrm{Co6} / \mathrm{TiO}_{2}$ had sintered less than IWI-Co/ $/ \mathrm{TiO}_{2}$ after FT, with final particles sizes of 11 and $14 \mathrm{~nm}$, respectively (Appendix C, Figure C12). Because the average particle sizes of both samples were initially comparable while the polydispersity of IWI-Co/ $\mathrm{TiO}_{2}$ was higher, this shows the impact of the width of the particle size distribution on the stability of the cobalt nanoparticles. ${ }^{62-64}$ It nicely illustrates the advantage of the NC-based approach over the traditional synthesis techniques to arrive at more stable catalysts.

On average, $12 \mathrm{~nm} \mathrm{Co-NC}$ on $\mathrm{TiO}_{2}$ did not grow but the particle size distribution did become broader already after reduction (Figure 4.4). Both smaller and larger particles were thus formed, which implied Ostwald ripening or re-dispersion of cobalt, possibly via cobalt-ion migration, under reducing conditions. No evidence of $\mathrm{Co}-\mathrm{NC}$ growth or redispersion was apparent on $\mathrm{Co} 9 / \mathrm{SiO}_{2}$ indicating that Co mobility was more extensive with the $\mathrm{TiO}_{2}$ support or the somewhat larger Co-NC sizes. Extending the reduction to $16 \mathrm{~h}$ instead of $8 \mathrm{~h}$ did not change the particle size distribution further (Appendix C, Figure C13). This observation is qualitatively in line with the results of Xaba et al. ${ }^{65}$ on titania P25, although their Co particles were substantially larger $(\sim 50 \mathrm{~nm})$. 
The size evolution of individual $\mathrm{Co}-\mathrm{NC}$ in $\mathrm{Co} 12 / \mathrm{TiO}_{2}$ was followed before and after reduction and passivation to investigate the re-dispersion in more detail (Figure 4.5). No particles were found that had moved from their original position, indicating low mobility of the $\mathrm{Co}-\mathrm{NC}$ as a whole and making migration and coalescence unlikely as a pathway for particle growth during reduction. Furthermore, approximately $5 \%$ of the particles broke up into multiple smaller particles (Figure 4.5c,d highlighted area, Appendix C, Figure C14). This re-dispersion caused an increase in the fraction of 5-8 $\mathrm{nm}$ particles, resulting in broadening of the particle size distribution.
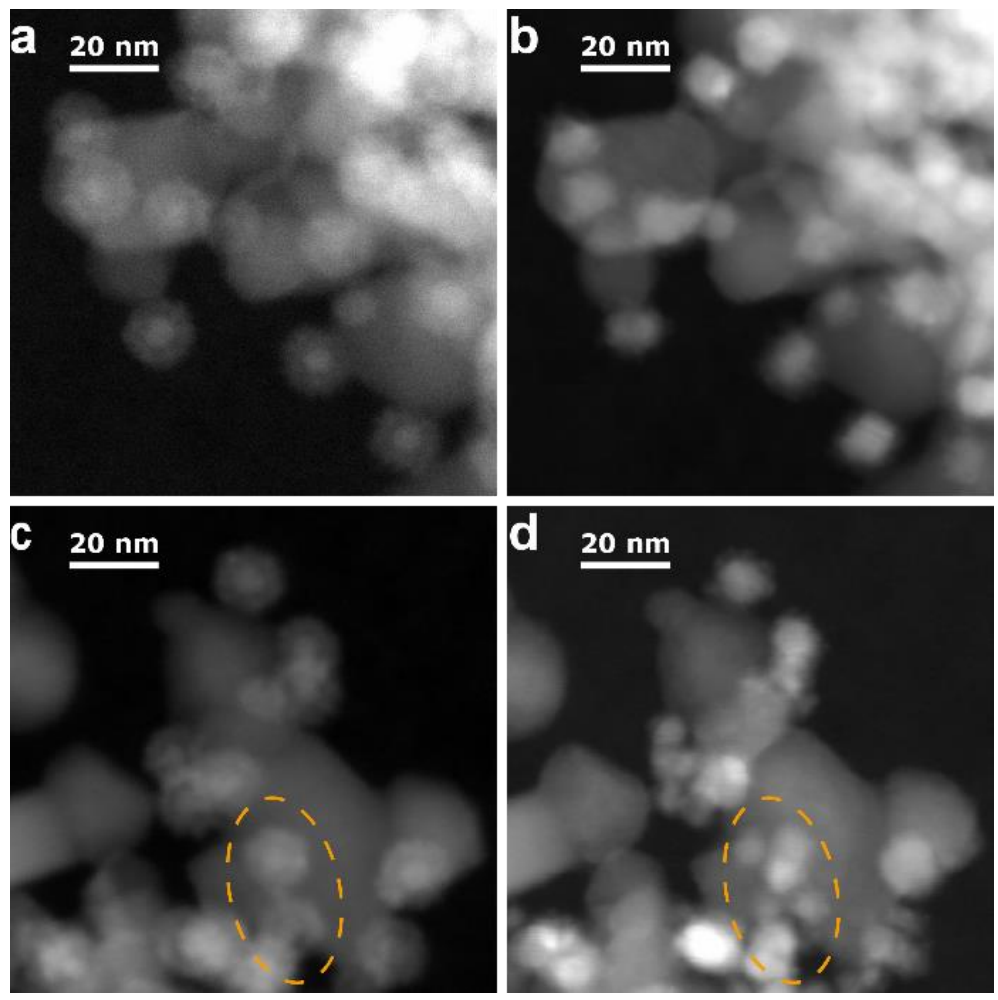

Figure 4.5. The size evolution of individual $\mathrm{Co}-\mathrm{NC}$ in $\mathrm{Co} 12 / \mathrm{TiO}_{2}$ during reduction. a,c HAADF-STEM images of pristine $\mathrm{Co12} / \mathrm{TiO}_{2}$ and $\mathbf{b}, \mathbf{d}$ HAADF-STEM images of the same locations after reduction at $350{ }^{\circ} \mathrm{C}$ for $8 \mathrm{~h}$. The highlighted area contains two particles that disintegrated during reduction and formed multiple smaller particles.

Two reasons can be proposed for the disintegration of the NC. First, it might be favorable for certain particles to maximize their contact area with the support, since the interaction between metals and reducible supports such as $\mathrm{TiO}_{2}$ can lead to dynamic systems under reducing conditions. ${ }^{38}$ Second, the core-shell structure in pristine $\mathrm{Co12} / \mathrm{TiO}_{2}$ could be an intermediate stage of the Kirkendall effect. ${ }^{6}$ Sasdavian et al. ${ }^{39}$ investigated this effect for $\mathrm{Co}-\mathrm{NC}$ of different sizes on $\mathrm{SiO}_{2}$ and reported similar core-shell particles in the case of incomplete oxidation of $29 \mathrm{~nm}$ particles. Completely oxidized, hollow cobalt oxide particles of $29 \mathrm{~nm}$ disintegrated into multiple smaller particles upon reduction, whereas smaller hollow particles returned to their original size of $11 \mathrm{~nm}$. Based

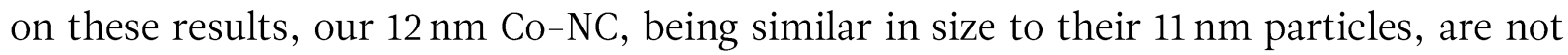
expected to disintegrate upon reduction. 


\subsection{Conclusion}

Here, we investigated the preparation of well-defined $\mathrm{TiO}_{2}-$ and $\mathrm{SiO}_{2}$-supported catalyst via colloidal synthesis of $\mathrm{Co}-\mathrm{NC}$ and evaluated their catalytic performance in the FischerTropsch synthesis. 3-12 nm Co-NC were synthesized by a hot-injection method, oxidized at low temperature to restrict magnetic interactions and subsequently attached to $\mathrm{TiO}_{2}$ or $\mathrm{SiO}_{2}$. The catalytic activity of most $\mathrm{TiO}_{2}$-supported samples was in line with that of a $\mathrm{Co} / \mathrm{TiO}_{2}$ sample prepared by impregnation of cobalt nitrate, proving that relevant catalytic data was obtained with these model catalysts. The cobalt-weight based activity of $3 \mathrm{~nm}$ $\mathrm{Co}-\mathrm{NC}$ on $\mathrm{TiO}_{2}$ was five-fold lower than expected and this was partially ascribed to loss of cobalt to the support. Furthermore, the $\mathrm{TiO}_{2}$-supported Co particles and $3 \mathrm{~nm} \mathrm{Co-NC} \mathrm{on}$ $\mathrm{SiO}_{2}$ sintered, while 6 and $9 \mathrm{~nm} \mathrm{Co-NC}$ on $\mathrm{SiO}_{2}$ were stable during reduction and FT. We showed that the interaction between $\mathrm{Co}(\mathrm{O})$ and $\mathrm{TiO}_{2}$ was responsible for the decreased stability of the Co-NC. This study illustrates the promise of synthesizing model catalysts using colloidal techniques, as the high precision in particle size enabled the investigation of effects that have not have been revealed earlier with conventional synthesis techniques.

\section{Acknowledgements}

T.W.v.D and K.P.d.J. acknowledge Shell Global Solutions and the Netherlands Association for Scientific Research (NWO) for funding through the CHIPP framework. N.A.K., J.Z. and K.P.d.J. acknowledge the European Research Council, EU FP7 ERC Advanced Grant no. 338846. We thank Pasi Paalanen and Lennart Weber ( $\mathrm{N}_{2}$-physisorption), Carlos Hernández Mejía (TPR), and Helen de Waard (ICP-OES) for the measurements indicated.

\section{References}

1. Zaera, F. Nanostructured materials for applications in heterogeneous catalysis. Chem. Soc. Rev. 42, 2746-2762 (2013).

2. Jia, C.-J. \& Schüth, F. Colloidal metal nanoparticles as a component of designed catalyst. Phys. Chem. Chem. Phys. 13, 2457-2487 (2011).

3. Beaumont, S. K. Recent developments in the application of nanomaterials to understanding molecular level processes in cobalt catalysed Fischer-Tropsch synthesis. Phys. Chem. Chem. Phys. 16, 5034-5043 (2014).

4. Werner, S., Johnson, G. R. \& Bell, A. T. Synthesis and Characterization of Supported CobaltManganese Nanoparticles as Model Catalysts for Fischer-Tropsch Synthesis. ChemCatChem 6, 2881-2888 (2014).

5. Zacharaki, E. et al. From colloidal monodisperse nickel nanoparticles to well-defined $\mathrm{Ni} / \mathrm{Al}_{2} \mathrm{O}_{3}$ model catalysts. Langmuir 33, 9836-9843 (2017).

6. Yin, Y. et al. Formation of Hollow Nanocrystals Through the Nanoscale Kirkendall Effect. Science 304, 711-714 (2004). 
7. van Deelen, T. W., Su, H., Sommerdijk, N. A. J. M. \& de Jong, K. P. Assembly and activation of supported cobalt nanocrystal catalysts for the Fischer-Tropsch synthesis. Chem. Commun. 54, 2530-2533 (2018).

8. Bezemer, G. L. et al. Cobalt Particle Size Effects in the Fischer-Tropsch Reaction Studied with Carbon Nanofiber Supported Catalysts. J. Am. Chem. Soc. 128, 3956-3964 (2006).

9. den Breejen, J. P. et al. On the Origin of the Cobalt Particle Size Effects in Fischer-Tropsch Catalysis. J. Am. Chem. Soc. 131, 7197-7203 (2009).

10. Sadeqzadeh, M. et al. Identification of the active species in the working alumina-supported cobalt catalyst under various conditions of Fischer-Tropsch synthesis. Catal. Today 164, 62-67 (2011).

11. Liu, J.-X., Su, H.-Y., Sun, D.-P., Zhang, B.-Y. \& Li, W.-X. Crystallographic Dependence of CO Activation on Cobalt Catalysts: HCP versus FCC. J. Am. Chem. Soc. 135, 16284-16287 (2013).

12. Lyu, S. et al. Role of Active Phase in Fischer-Tropsch Synthesis: Experimental Evidence of CO Activation over Single-Phase Cobalt Catalysts. ACS Catal. 8, 7787-7798 (2018).

13. Gual, A., Godard, C., Castillón, S., Curulla-Ferré, D. \& Claver, C. Colloidal Ru, Co and Fenanoparticles. Synthesis and application as nanocatalysts in the Fischer-Tropsch process. Catal. Today 183, 154-171 (2012).

14. Wang, H. et al. Platinum-Modulated Cobalt Nanocatalysts for Low-Temperature AqueousPhase Fischer-Tropsch Synthesis. J. Am. Chem. Soc. 135, 4149-4158 (2013).

15. Delgado, J. A., Claver, C., Castillón, S., Curulla-Ferré, D. \& Godard, C. Correlation between Hydrocarbon Product Distribution and Solvent Composition in the Fischer-Tropsch Synthesis Catalyzed by Colloidal Cobalt Nanoparticles. ACS Catal. 5, 4568-4578 (2015).

16. Prieto, G., Martínez, A., Concepción, P. \& Moreno-Tost, R. Cobalt particle size effects in Fischer-Tropsch synthesis: structural and in situ spectroscopic characterisation on reverse micelle-synthesised Co/ITQ-2 model catalysts. J. Catal. 266, 129-144 (2009).

17. Fischer, N., van Steen, E. \& Claeys, M. Structure sensitivity of the Fischer-Tropsch activity and selectivity on alumina supported cobalt catalysts. J. Catal. 299, 67-80 (2013).

18. Lögdberg, S. et al. Effect of water on the space-time yield of different supported cobalt catalysts during Fischer-Tropsch synthesis. Appl. Catal. A Gen. 393, 109-121 (2011).

19. Ralston, W. T., Melaet, G., Saephan, T. \& Somorjai, G. A. Evidence of Structure Sensitivity in the Fischer-Tropsch Reaction on Model Cobalt Nanoparticles by Time-Resolved Chemical Transient Kinetics. Angew. Chemie Int. Ed. 56, 7415-7419 (2017).

20. Melaet, G., Lindeman, A. E. \& Somorjai, G. A. Cobalt Particle Size Effects in the Fischer-Tropsch Synthesis and in the Hydrogenation of $\mathrm{CO}_{2}$ Studied with Nanoparticle Model Catalysts on Silica. Top. Catal. 57, 500-507 (2014).

21. Herranz, T., Deng, X., Cabot, A., Guo, J. \& Salmeron, M. Influence of the Cobalt Particle Size in the CO Hydrogenation Reaction Studied by In Situ X-Ray Absorption Spectroscopy. J. Phys. Chem. B113, 10721-10727 (2009).

22. Lee, Y.-J., Park, J.-Y., Jun, K.-W., Wook Bae, J. \& Prasad, P. S. S. Controlled Nanocrystal Deposition for Higher Degree of Reduction in $\mathrm{Co} / \mathrm{Al}_{2} \mathrm{O}_{3}$ Catalyst. Catal. Letters 130, 198-203 (2009). 
23. Park, J.-Y. et al. Fischer-Tropsch catalysts deposited with size-controlled $\mathrm{Co}_{3} \mathrm{O}_{4}$ nanocrystals: Effect of Co particle size on catalytic activity and stability. Appl. Catal. A Gen. 411-412, 15-23 (2012).

24. Oukaci, R., Singleton, A. H. \& Goodwin, J. G. Comparison of patented Co F-T catalysts using fixed-bed and slurry bubble column reactors. Appl. Catal. A Gen. 186, 129-144 (1999).

25. Morales, F., de Smit, E., de Groot, F. M. F., Visser, T. \& Weckhuysen, B. M. Effects of manganese oxide promoter on the $\mathrm{CO}$ and $\mathrm{H}_{2}$ adsorption properties of titania-supported cobalt FischerTropsch catalysts. J. Catal. 246, 91-99 (2007).

26. Tauster, S. J., Fung, S. C. \& Garten, R. L. Strong Metal-Support Interactions. Group 8 Noble Metals Supported on $\mathrm{TiO}_{2}$. J. Am. Chem. Soc. 100, 170-175 (1978).

27. Tauster, S. J., Fung, S. C., Baker, R. T. \& Horsley, J. A. Strong Interactions in Supported-Metal Catalysts. Science 211, 1121-1125 (1981).

28. Eschemann, T. O. \& de Jong, K. P. Deactivation Behavior of $\mathrm{Co} / \mathrm{TiO}_{2}$ Catalysts during FischerTropsch Synthesis. ACS Catal. 5, 3181-3188 (2015).

29. Hong, J. et al. Plasma-assisted Preparation of Highly Dispersed Cobalt Catalysts for Enhanced Fischer-Tropsch Synthesis Performance. ACS Catal. 8, 6177-6185 (2018).

30. Delgado, J. A. et al. Fischer-Tropsch synthesis catalysed by small $\mathrm{TiO}_{2}$ supported cobalt nanoparticles prepared by sodium borohydride reduction. Appl. Catal. A Gen. 513, 39-46 (2016).

31. Delgado, J. A. et al. Effect of polymeric stabilizers on Fischer-Tropsch synthesis catalyzed by cobalt nanoparticles supported on $\mathrm{TiO}_{2}$. J. Mol. Catal. A Chem. 417, 43-52 (2016).

32. Melaet, G. et al. Evidence of Highly Active Cobalt Oxide Catalyst for the Fischer-Tropsch Synthesis and $\mathrm{CO}_{2}$ Hydrogenation. J. Am. Chem. Soc. 136, 2260-2263 (2014).

33. Iablokov, V. et al. Size-Controlled Model Co Nanoparticle Catalysts for $\mathrm{CO}_{2}$ Hydrogenation: Synthesis, Characterization, and Catalytic Reactions. Nano Lett. 12, 3091-3096 (2012).

34. Casavola, M., Hermannsdörfer, J., de Jonge, N., Dugulan, A. I. \& de Jong, K. P. Fabrication of Fischer-Tropsch Catalysts by Deposition of Iron Nanocrystals on Carbon Nanotubes. Adv. Funct. Mater. 25, 5309-5319 (2015).

35. Reuel, R. C. \& Bartholomew, C. H. The Stoichiometries of $\mathrm{H}_{2}$ and CO Adsorptions on Cobalt: Effects of Support and Preparation. J. Catal. 85, 63-77 (1984).

36. Zacharaki, E., Kalyva, M., Fjellvåg, H. \& Sjåstad, A. O. Burst nucleation by hot injection for size controlled synthesis of $\varepsilon$-cobalt nanoparticles. Chem. Cent. J. 10,10 (2016).

37. Wolf, M., Fischer, N. \& Claeys, M. Effectiveness of catalyst passivation techniques studied in situ with a magnetometer. Catal. Today 275, 135-140 (2016).

38. Hernández Mejía, C., van Deelen, T. W. \& de Jong, K. P. Activity enhancement of cobalt catalysts by tuning metal-support interactions. Nat. Commun. 9, 4459 (2018).

39. Sadasivan, S., Bellabarba, R. M. \& Tooze, R. P. Size dependent reduction-oxidation-reduction behaviour of cobalt oxide nanocrystals. Nanoscale 5, 11139-11146 (2013).

40. Wolf, M., Kotzé, H., Fischer, N. \& Claeys, M. Size dependent stability of cobalt nanoparticles in high conversion Fischer-Tropsch environment. Faraday Discuss. 197, 243-268 (2017).

41. van Steen, E. et al. Stability of Nanocrystals: Thermodynamic Analysis of Oxidation and Rereduction of Cobalt in Water/Hydrogen Mixtures. J. Phys. Chem. B109, 3575-3577 (2005). 
42. Tsakoumis, N. E. et al. Evaluation of Reoxidation Thresholds for $\gamma-\mathrm{Al}_{2} \mathrm{O}_{3}$-Supported Cobalt Catalysts under Fischer-Tropsch Synthesis Conditions. J. Am. Chem. Soc. 139, 3706-3715 (2017).

43. Prieto, G. et al. Cobalt-Catalyzed Fischer-Tropsch Synthesis: Chemical Nature of the Oxide Support as a Performance Descriptor. ACS Catal. 5, 3323-3335 (2015).

44. Yang, J., Tveten, E. Z., Chen, D. \& Holmen, A. Understanding the Effect of Cobalt Particle Size on Fischer-Tropsch Synthesis: Surface Species and Mechanistic Studies by SSITKA and Kinetic Isotope Effect. Langmuir 26, 16558-16567 (2010).

45. Kliewer, C. E., Soled, S. L. \& Kiss, G. Morphological transformations during Fischer-Tropsch synthesis on a titania-supported cobalt catalyst. Catal. Today 323, 233-256 (2019).

46. Iglesia, E., Soled, S. L. \& Fiato, R. A. Fischer-Tropsch Synthesis on Cobalt and Ruthenium. Metal Dispersion and Support Effects on Reaction Rate and Selectivity. J. Catal. 137, 212-224 (1992).

47. Iglesia, E. Design, synthesis, and use of cobalt-based Fischer-Tropsch synthesis catalysts. Appl. Catal. A Gen. 161, 59-78 (1997).

48. Storsæter, S., Borg, Ø., Blekkan, E. A. \& Holmen, A. Study of the effect of water on FischerTropsch synthesis over supported cobalt catalysts. J. Catal. 231, 405-419 (2005).

49. Munnik, P., de Jongh, P. E. \& de Jong, K. P. Control and Impact of the Nanoscale Distribution of Supported Cobalt Particles used in Fischer-Tropsch Catalysis. J. Am. Chem. Soc. 136, 73337340 (2014).

50. Eschemann, T. O. et al. Effect of support surface treatment on the synthesis, structure, and performance of Co/CNT Fischer-Tropsch catalysts. J. Catal. 328, 130-138 (2015).

51. Campbell, C. T. \& Mao, Z. Chemical Potential of Metal Atoms in Supported Nanoparticles: Dependence upon Particle Size and Support. ACS Catal. 7, 8460-8466 (2017).

52. Munnik, P., Velthoen, M. E. Z., de Jongh, P. E., De Jong, K. P. \& Gommes, C. J. Nanoparticle Growth in Supported Nickel Catalysts during Methanation Reaction-Larger is Better. Angew. Chemie Int. Ed. 53, 9493-9497 (2014).

53. Ouyang, R., Liu, J.-X. \& Li, W.-X. Atomistic Theory of Ostwald Ripening and Disintegration of Supported Metal Particles under Reaction Conditions. J. Am. Chem. Soc. 135, 1760-1771 (2013).

54. Wilson, J. \& de Groot, C. P. M. Atomic-Scale Restructuring in High-Pressure Catalysis. J. Phys. Chem. 99, 7860-7866 (1995).

55. Claeys, M. et al. Impact of Process Conditions on the Sintering Behavior of an AluminaSupported Cobalt Fischer-Tropsch Catalyst Studied with an in Situ Magnetometer. ACS Catal. 5, 841-852 (2015).

56. Janse van Rensburg, W. et al. Role of Transient Co-Subcarbonyls in Ostwald Ripening Sintering of Cobalt Supported on $\curlyvee$-Alumina Surfaces. J. Phys. Chem. C121, 16739-16753 (2017).

57. Jongsomjit, B., Sakdamnuson, C., Goodwin, J. G. \& Praserthdam, P. Co-support compound formation in titania-supported cobalt catalyst. Catal. Letters 94, 209-215 (2004).

58. Cats, K. H. et al. Active phase distribution changes within a catalyst particle during FischerTropsch synthesis as revealed by multi-scale microscopy. Catal. Sci. Technol. 6, 4438-4449 (2016).

59. Riva, R., Miessner, H., Vitali, R. \& Del Piero, G. Metal-support interaction in $\mathrm{Co} / \mathrm{SiO}_{2}$ and Co/TiO2. Appl. Catal. A Gen. 196, 111-123 (2000). 
60. Wang, C.-B., Cai, Y. \& Wachs, I. E. Reaction-Induced Spreading of Metal Oxides onto Surfaces of Oxide Supports during Alcohol Oxidation: Phenomenon, Nature, and Mechanisms. Langmuir 15, 1223-1235 (1999).

61. Kistamurthy, D., Saib, A. M., Moodley, D. J., Niemantsverdriet, J. W. \& Weststrate, C. J. Ostwald ripening on a planar $\mathrm{Co} / \mathrm{SiO}_{2}$ catalyst exposed to model Fischer-Tropsch synthesis conditions. J. Catal. 328, 123-129 (2015).

62. Hu, S. \& Li, W.-X. Influence of Particle Size Distribution on Life Time and Thermal Stability of Ostwald Ripening of Supported Particles. ChemCatChem 10, 2900-2907 (2018).

63. Wettergren, K. et al. High Sintering Resistance of Size-Selected Platinum Cluster Catalysts by Suppressed Ostwald Ripening. Nano Lett. 14, 5803-5809 (2014).

64. Zhang, S. et al. Revealing particle growth mechanisms by combining high-surface-area catalysts made with monodisperse particles and electron microscopy conducted at atmospheric pressure. J. Catal. 337, 240-247 (2016).

65. Xaba, B. M. \& de Villiers, J. P. R. Sintering behaviour of $\mathrm{TiO}_{2}$-supported model cobalt FischerTropsch catalysts under $\mathrm{H}_{2}$ reducing conditions and elevated temperature. Ind. Eng. Chem. Res. 55, 9397-9407 (2016). 







\subsection{Introduction}

Controlled synthesis of supported metal nanoparticles is one of the main challenges in designing heterogenous catalysts for major industrial processes as well as fundamental research. ${ }^{1-3}$ Traditional catalyst synthesis methods have in common that nanoparticle formation takes place on the support. ${ }^{4}$ Recent advances in catalyst synthesis include the use of colloidal techniques to synthesize nanocrystals (NC) in solution followed by attachment to a support. ${ }^{4,5}$ By separating the NC synthesis from attachment to the support, the properties of the NC can be manipulated with greater precision, leading for example to NC of particular sizes, crystal structures and shapes. ${ }^{6}$ Among these properties, nanoparticle shape is arguably the hardest to tune using conventional techniques, so here colloidal methods could offer a clear advantage over existing catalyst synthesis techniques, provided that challenges regarding $\mathrm{NC}$ attachment to the support and ligand removal are solved.?

Anisotropic NC are often metastable compared to spherical NC, which are usually the equilibrium shape in particular in the case of metals. ${ }^{8,9}$ By using ligands that bind preferentially to specific surface facets, it is possible to inhibit growth in certain directions and to drive the process towards a kinetically controlled product. ${ }^{10,11}$ Distinct crystal facets are exposed at the surface of these particles, which may lead to distinct catalytic performance. Additionally, different shapes also imply different relative amounts of corner, edge and terrace sites, the reactivity of which can vary depending on the type of reaction. ${ }^{12}$ The effect of NC shape on catalytic performance has already been investigated mainly for noble metal ${ }^{13-15}$ or for metal oxide $\mathrm{NC},{ }^{16}$ yet few accounts of shape variation for supported base metal NC catalysts exist.

The shape of NC plays an important role if the catalyzed reaction is structure sensitive. One pronounced example of such a reaction is the Fischer-Tropsch (FT) synthesis. ${ }^{12}$ In FT, a mixture of $\mathrm{H}_{2}$ and $\mathrm{CO}$ called synthesis gas is converted into hydrocarbon fuels and chemicals over a catalyst, typically based on Fe or Co. ${ }^{17}$ The cobalt-catalyzed FT reaction is sensitive to various structural aspects of the Co particles. ${ }^{18}$ For example, a size dependency of the catalytic performance of cobalt is generally reported, with the surface-specific activity and $\mathrm{C}_{5_{+}}$-selectivity decreasing sharply below a particle size of $6-8 \mathrm{~nm} \cdot{ }^{19-21}$ Additionally, several studies indicated that the crystal structure of Co is relevant, with hcp being more active than $\mathrm{fcc},{ }^{22-26}$ although this topic remains controversial. ${ }^{27}$ Given the potential influence of the crystal structure, the shape of Co particles might be important as well, as shape partially governs the surface structure of nanoparticles.

For the potential effect of the NC shape on catalytic FT performance we should resort mainly to theoretical and surface science studies, as little experimental data is available on complex model catalysts under high-pressure conditions. Theoretical studies predict that the B5 surface ensemble is essential for CO-dissociation, which is considered an important step for activity and selectivity. The occurrence of coordinatively unsaturated sites, including B5 sites, varies with particle size ${ }^{28,29}$ and crystal structure ${ }^{29}$ and offers one explanation for the observed structure sensitivity. Furthermore, a computational comparison of the FT activity of various Co crystal planes indicated that higher index planes of hcp Co are orders of magnitude more active than the close packed (0001). ${ }^{25}$ Surface science experiments on single crystal planes of hep Co showed that relatively more $\mathrm{CH}_{4}$ is 

under industrially relevant conditions

produced on hcp (0001) than on higher index planes, leading to a lower selectivity towards $\mathrm{C}_{5+}$ - products. $^{30,31}$ However, a recent experimental study indicated that hep (0001) displayed intermediate activity and $\mathrm{C}_{5_{+}}$-selectivity, with both increasing in the order hcp $\{11 \overline{20}\}>\{0001\}>\{10 \overline{11}\} .{ }^{32}$ All of these aspects of surface composition could in principle be affected by the shape of the Co-NC, although it is also possible that surface defects, ${ }^{33}$ hydrocarbon coverage ${ }^{34}$ or $\mathrm{CO}$-induced surface reconstruction ${ }^{35-41}$ might in practice annihilate the effects of $\mathrm{Co}-\mathrm{NC}$ shape.

Surface science cannot always be directly translated to more complex catalysts and high-pressure systems. ${ }^{5}$ To bridge the gap between surface science and supported catalysts under industrially relevant reaction conditions, 3D model catalysts containing anisotropic Co particles are required. Examples of anisotropic cobalt nanoparticles in FT include unsupported particles, such as $\mathrm{CoMnO}_{\mathrm{x}}$ nanoprisms, ${ }^{42}$ Co nanocubes, ${ }^{43}$ bimetallic nanorods ${ }^{44}$ and $\mathrm{Co}_{3} \mathrm{O}_{4}$ nanorods, ${ }^{45}$ plates or cubes ${ }^{32}$. Furthermore, hcp nanorods supported either on $\mathrm{SiO}_{2}-\mathrm{Al}_{2} \mathrm{O}_{3}{ }^{46}$ or on $\mathrm{Ni}$ or $\mathrm{Cu}$ foams ${ }^{47}$ have also been employed. Clearly, synthetic approaches are still required to produce anisotropic model systems that closely resemble conventional supported cobalt catalyst, with stable Co-NC shapes yet without additives, such as a second metal, that might influence the FT performance.

In this chapter, we report our efforts to advance the synthesis, characterization and testing of shape-controlled Co-NC dispersed on a support as relevant model catalysts for the Fischer-Tropsch synthesis. We consistently obtained a mixture of disk-shaped and spherical Co-NC. The disks were $15 \mathrm{~nm}$ in diameter with a thickness of $4 \mathrm{~nm}$ and consisted of hcp Co with (0001) terminating the base planes, while the spherical particles consisted of $\varepsilon$-Co. After low-temperature oxidation of the $\mathrm{Co}-\mathrm{NC}$, the $\mathrm{CoO}-\mathrm{NC}$ were attached to $\mathrm{SiO}_{2}$ $\left(50 \mathrm{~m}^{2} \cdot \mathrm{g}^{-1}\right)$. Numerically $66 \%$ of the NC were disks, leading to a higher occurrence of hcp (0001) on the cobalt surface compared to purely spherical Co-NC. The FT activity of the disks/spheres mixture was half that of spheres only, while the $\mathrm{C}_{5_{+}}$-selectivity was identical. Remarkably, the shape endured the FT conditions, as $62 \%$ of the NC after catalysis were disks of the original dimensions. Nitrogen-containing ligands were largely removed, and both catalysts featured the same crystalline composition, leaving the possibility for enhanced hcp (0001) exposure in disks. Based on these experiments, it was concluded that the disk shape of Co-NC had a strong influence on the FT performance, most probably caused by the introduction of a high number inactive low-index Co planes.

\subsection{Experimental}

\subsubsection{Synthesis of disk-shaped nanocrystals}

A mixture of disk-shaped and spherical Co-NC was synthesized based on an adapted procedure originally developed by Puntes et al. ${ }^{10,48}$ The ligands, $50 \mathrm{mg}$ oleic acid $(90 \%$ technical grade, Sigma-Aldrich) and $600 \mathrm{mg}$ octadecylamine (>99.0\% (GC), SigmaAldrich), were transferred to a three-necked $100 \mathrm{~mL}$ flask with two septa and a reflux condenser connected to a Schlenk line. The mixture was heated to $80{ }^{\circ} \mathrm{C}$ under vacuum using a heating mantle while magnetically stirring at $600 \mathrm{rpm}$. After $30 \mathrm{~min}$, the setup was flushed three times with $\mathrm{N}_{2}$ before switching to $\mathrm{N}_{2}$ for the remainder of the synthesis. Then, 
$7.5 \mathrm{~mL}$ 1,2-dichlorobenzene (99\% Anhydrous, Sigma-Aldrich) was added to the mixture without air-exposure, a thermocouple was inserted and the mixture was heated to reflux temperature $\left(188^{\circ} \mathrm{C}\right)$. Meanwhile, $270 \mathrm{mg}$ dicobalt octacarbonyl (95\%, stabilized with hexane, Acros Organics) was transferred to a vial in an $\mathrm{N}_{2}$-glovebox and the vial was left open inside the glovebox for 40 min to age. After ageing, the $\mathrm{Co}_{2}(\mathrm{CO})_{8}$ was dissolved in $1.5 \mathrm{~mL}$ 1,2-dichlorobenzene and the vial was closed using a cap with a septum and removed from the glovebox. Once the liquid was at reflux temperature, the cobalt precursor was rapidly injected into the center of the flask using a syringe with a $\varnothing 1.2 \mathrm{x} 40 \mathrm{~mm}$ needle under $600 \mathrm{rpm}$ stirring. The synthesis was quenched after 5 min using a water bath. The water bath was removed after $\sim 3$ min when the temperature of the reaction mixture was below $30^{\circ} \mathrm{C}$. Simultaneously, the stirring was stopped and the stirring plate placed $5 \mathrm{~cm}$ below the flask to magnetically separate the reaction product. The top $7 \mathrm{~mL}$ contained the highest concentration of disk-shaped $\mathrm{Co}-\mathrm{NC}$ and was transferred to a glass centrifuge tube after 5 min and exposed to air from this point on. Consequently, the NC were oxidized to $\mathrm{CoO}$ to facilitate their uniform distribution over a support at a later stage. ${ }^{7}$ The centrifuge tube was filled to $20 \mathrm{~mL}$ with 2-propanol ( $>99.5 \%$ (GC), Merck) and centrifuged at $2500 \mathrm{G}$ for $10 \mathrm{~min}$. The purple/brown supernatant was decanted and the residue redispersed in $0.5 \mathrm{~mL} \mathrm{n}$-hexane (99\%, Acros Organics) using sonication. Another precipitationredispersion cycle was performed by filling the tube to $20 \mathrm{~mL}$ with 2-propanol, centrifuging at $2500 \mathrm{G}$ for $40 \mathrm{~min}$, decanting the supernatant and finally redispersing the residue in $10 \mathrm{~mL}$-hexane using sonication.

\subsubsection{Attachment of disk-shaped nanocrystals}

The disk-shaped $\mathrm{CoO}-\mathrm{NC}$ were attached to $\mathrm{SiO}_{2}$ (Aerosil OX 50, Evonik) within $4 \mathrm{~h}$ after synthesis. To obtain enough material with sufficient metal loading, two NC synthesis batches were attached consecutively to the same batch of support. A glass round bottom vessel with an overhead mechanical stirrer was placed in a sonication bath. $300 \mathrm{mg} \mathrm{\textrm {SiO } _ { 2 }}$ and $20 \mathrm{~mL} \mathrm{n}$-hexane were added and the top of the vessel was loosely covered with aluminum foil. Stirring and sonication were applied to suspend the $\mathrm{SiO}_{2}$ for 20 min and then the first $\mathrm{CoO}-\mathrm{NC}$ batch in $10 \mathrm{~mL} \mathrm{n}$-hexane was added dropwise in $15 \mathrm{~min}$ under continued stirring and sonication. The mixture was left for $45 \mathrm{~min}$ after the addition and then the $\mathrm{n}$ hexane was slowly evaporated in $15 \mathrm{~min}$ by a applying a flow of $\mathrm{N}_{2}$ through the vessel, all under sonication and stirring. This entire procedure was repeated a second time by suspending the dried uniformly grey powder again in $20 \mathrm{~mL} \mathrm{n}$-hexane and adding the second batch of $\mathrm{CoO}-\mathrm{NC}$. Afterwards, the dried powder was suspended in $10 \mathrm{~mL}$-hexane and transferred to a glass centrifuge tube. The solid was precipitated by centrifuging at $2500 \mathrm{G}$ for $1 \mathrm{~min}$ and the supernatant was decanted. The residue was 5 times resuspended in $5 \mathrm{~mL} \mathrm{n}$-hexane and precipitated by centrifuging at $2500 \mathrm{G}$ for $1 \mathrm{~min}$ and finally dried at ambient temperature under vacuum for $16 \mathrm{~h}$. The dry powder was pressed and sieved to a grain size of 75-150 $\mathrm{mm}$ (100 mg was obtained).

\subsubsection{Synthesis of spherical nanocrystals}

Exclusively spherical Co-NC were synthesized according to a comparable hot-injection method, however, only oleic acid was used as ligand according to a procedure described 
before ${ }^{49}$. The synthesis was performed in $\mathrm{N}_{2}$ atmosphere using a Schlenk line. $65 \mathrm{mg}$ oleic acid was degassed under vacuum at $100{ }^{\circ} \mathrm{C}$ for 30 min under $650 \mathrm{rpm}$ magnetic stirring in a 3-necked $100 \mathrm{~mL}$ flask with a condenser and two septa. The flask was flushed with $\mathrm{N}_{2}$ and the synthesis was continued under $\mathrm{N}_{2}$. Then, $7.5 \mathrm{~mL}$ 1,2-dichlorobenzene was added under $\mathrm{N}_{2}$ atmosphere and the solution was heated to $165^{\circ} \mathrm{C}$. Meanwhile, $270 \mathrm{mg}$ dicobalt octacarbonyl was dissolved in $1.5 \mathrm{~mL} \mathrm{1,2-dichlorobenze} \mathrm{and} \mathrm{sealed} \mathrm{under} \mathrm{N}_{2}$ atmosphere inside a glovebox. This cobalt precursor was then rapidly injected (needle: $\varnothing 0.9 \times 70 \mathrm{~mm}$ ) into the pre-heated solution at $750 \mathrm{rpm}$ stirring and reacted vigorously. After $20 \mathrm{~min}$, the reaction was quenched and cooled to at ambient temperature using a water bath. One septum was then removed to oxidize the $\mathrm{Co}-\mathrm{NC}$ to $\mathrm{CoO}-\mathrm{NC}$ by air-exposure at low temperature for $1 \mathrm{~h}$ (650 rpm stirring). The suspension was divided after over two glass centrifuge tubes, which were filled further to $20 \mathrm{~mL}$ with 2-propanol and centrifuged at $2200 \mathrm{G}$ for $30 \mathrm{~min}$ to precipitate the NC. The supernatant was decanted, the precipitate redispersed in $0.5 \mathrm{~mL} \mathrm{n}$-hexane by sonication and the tubes were filled again to $20 \mathrm{~mL}$ with 2-propanol and centrifuged at $2200 \mathrm{G}$ for $40 \mathrm{~min}$. This precipitation-redispersion cycle was repeated two more times and afterwards, the residue was redispersed and combined in $2 \mathrm{~mL} \mathrm{n}$-hexane in total.

\subsubsection{Attachment of spherical nanocrystals}

The spherical CoO-NC were attached on the same type of low surface area $\mathrm{SiO}_{2}$ as the disk-

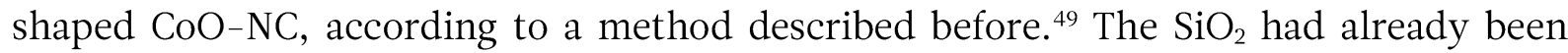
pressed and sieved to a fraction of $75-150 \mu \mathrm{m}$. The support $(500 \mathrm{mg})$ was transferred to a $100 \mathrm{~mL} 3$-necked flask and a mixture of the $\mathrm{Co}-\mathrm{NC}$ in $\mathrm{n}$-hexane and $6.5 \mathrm{~mL} 1$-octadecene (90\%, technical grade, Sigma-Aldrich) was added under $400 \mathrm{rpm}$ magnetic stirring. The flask was then equipped with a condenser, glass plug and septum and connected to a Schlenk line. The suspension was degassed under vacuum at $100{ }^{\circ} \mathrm{C}$ for $15 \mathrm{~min}$ and as a result, the $\mathrm{n}$-hexane in the suspension evaporated. Then, the flask was flushed with $\mathrm{N}_{2}$ and the attachment was continued under $\mathrm{N}_{2}$. The suspension was heated to $200{ }^{\circ} \mathrm{C}$ for $30 \mathrm{~min}$, after which the heating mantle was removed, causing the solution to cool to room temperature within $30 \mathrm{~min}$. The sample was washed by first centrifuging at $1500 \mathrm{G}$ for $5 \mathrm{~min}$ and decanting the supernatant. Subsequently, the precipitate was resuspended in $2 \mathrm{~mL} \mathrm{n}$-hexane through sonication, $6 \mathrm{~mL}$ acetone was added and the sample was isolated by centrifuging at $1500 \mathrm{G}$ for $5 \mathrm{~min}$ and decanting the supernatant. This precipitationresuspension cycle was repeated five times, followed by a last cycle in which the nhexane/acetone mixture was replaced by $20 \mathrm{~mL}$ acetone. Finally, the sample was dried in three consecutive steps, i.e. $60{ }^{\circ} \mathrm{C}$ for $1 \mathrm{~h}$ in static air, $120^{\circ} \mathrm{C}$ for $3 \mathrm{~h}$ in static air and $80{ }^{\circ} \mathrm{C}$ for $3 \mathrm{~h}$ under vacuum, and pressed and sieved to a grain size of 75-150 $\mu \mathrm{m}$.

\subsubsection{Characterization}

Transmission electron microscopy (TEM) was performed on a Tecnai 20 (FEI) operated at $200 \mathrm{kV}$. Samples of colloidal suspension were prepared by drop casting a diluted sample directly on a carbon-coated TEM grid. In the case of $\mathrm{SiO}_{2}$-supported $\mathrm{CoO}-\mathrm{NC}$, the powder was first suspended in 2-propanol by sonication and subsequently drop casted on a carboncoated TEM grid. The TEM images of supported CoO-NC were manually analyzed using 
ImageJ. In the case of the colloidal suspensions, the images of $\mathrm{CoO}-\mathrm{NC}$ were automatically classified as either disks or spheres and manually reviewed before analysis. All reported NC sizes are number averaged and recalculated to the equivalent $\mathrm{Co}^{0}$ size to compensate for lattice expansion due to a $3 \mathrm{~nm}$ thick $\mathrm{CoO}$ passivation layer. Because of the limited height of the disks, these $\mathrm{NC}$ were assumed to oxidize completely to $\mathrm{CoO}$. The metallic surface area was calculated using the surface area weighted mean diameter, again after correcting for the $3 \mathrm{~nm} \mathrm{CoO}$ passivation layer. A cross-sectional area of $0.0662 \mathrm{~nm}^{2}$ per cobalt atom was assumed in order to calculate the metallic surface area. ${ }^{50}$

Vitrification of freshly synthesized colloidal suspensions for cryogenic transmission electron microscopy (cryo-TEM) was performed using a Vitrobot Mark IV (FEI) vitrification robot. The $\mathrm{Co}-\mathrm{NC}$ were vitrified in the original reaction suspension within 30 min from magnetically separating the synthesis product. For the vitrification, $3 \mu \mathrm{L}$ of Co-NC suspension was applied to a 200 mesh, lacey carbon-coated $\mathrm{Cu}$ grid at $25^{\circ} \mathrm{C}$ in a chamber saturated with toluene vapor. Subsequently, the grid was blotted for $3 \mathrm{~s}$ with filter paper and plunge-frozen in liquid $\mathrm{N}_{2}\left(-196^{\circ} \mathrm{C}\right)$. The samples were kept in liquid $\mathrm{N}_{2}$ for several hours and loaded into the Titan Krios (FEI) equipped with a field emission gun operating at $300 \mathrm{kV}$ and energy filter. Images were recorded using a $2 \mathrm{k} \times 2 \mathrm{k}$ Gatan charge coupled device (CCD) camera. High-resolution transmission electron microscopy (HRTEM) was performed in areas where no solvent appeared present. Low dose selective area diffraction (SAED) was used for structure analysis and the diffraction patterns were processed according to the method described by Leijten et al. ${ }^{51}$

Electron tomography was carried out using an FEI Talos F200X operated at $200 \mathrm{kV}$ in bright field TEM mode. The $\mathrm{CoO}-\mathrm{NC} / \mathrm{SiO}_{2}$ sample was suspended in ethanol by ultrasonication. A few droplets of the suspension were deposited onto a Quantifoil R2/1 carbon film supported parallel-bar $\mathrm{Cu}$ grid which already contained Au nanoparticles of 5 $\mathrm{nm}$ in diameter. TEM images were recorded with a Ceta camera $(4096 \times 4096$ pixels $)$ over a tilt range of $-66^{\circ}$ to $+76^{\circ}$ with tilt increments of $2^{\circ}$ at a nominal magnification of 74,000 times. The tilt series was aligned using IMOD software ${ }^{52}$ using the Au nanoparticles as fiducial markers. The aligned tilt series were binned by 4 and reconstructed using the backprojection algorithm. The resulting reconstructed volumes had a final voxel size of $(0.572 \mathrm{~nm})^{3}$. Segmentation of disk-shaped $\mathrm{CoO}-\mathrm{NC}$, spherical $\mathrm{CoO}-\mathrm{NC}$ and the $\mathrm{SiO}_{2}$ support in the reconstructed volumes was performed in FIJI. ${ }^{53}$ Adequate threshold values were applied to median-filtered reconstructed volumes. Disk-shaped NC, spherical NC and the $\mathrm{SiO}_{2}$ support were defined in 3D by manually delimiting their boundary. Volume rendering of the segmented volumes was carried out in FIJI.

Inductively coupled plasma-optical emission spectroscopy (ICP-OES) was performed on a SPECTRO ARCOS after digestion of the sample in aqua regia.

X-ray photoelectron spectroscopy (XPS) was measured on a Kratos Axis Ultra DLD with an X-ray source operating at $225 \mathrm{~W}$ and $15 \mathrm{keV}$, and equipped with an $\mathrm{Al}$ anode $(\mathrm{EK} \alpha=1486.6 \mathrm{eV})$. The samples were fixed using Ag-tape at ambient conditions and loaded in the setup. High-resolution spectra of Co2p, Si2p, O1s and C1s were measured at $40 \mathrm{eV}$ pass energy, while N1s and the survey scan were recorded at $160 \mathrm{eV}$ pass energy. Kratos Axis sensitivity factors were used for quantification. XPS measurements were 
performed on three distinct locations on all samples and the results were averaged. In case of the N1s scan, multiple measurements of the same location were accumulated to improve the signal-to-noise ratio. The binding energies were calibrated by setting the $\mathrm{C}-\mathrm{C} / \mathrm{C}-\mathrm{H}$ bond peak to $284.8 \mathrm{eV}$.

In situ X-ray diffraction (XRD) was performed on a Bruker D8 Discover with a Mo $\left(\mathrm{K}_{\alpha 1} 0.709 \AA\right)$ source in Debye-Scherrer transmission (capillary) geometry. The $\mathrm{X}$-ray beam was focused on a quartz capillary with $1000 \mu \mathrm{m}$ OD and a wall thickness of $10 \mu \mathrm{m}$ using a Göbel-mirror. The setup was equipped with an energy dispersive LynxEye XE Position Sensitive Detector (PSD). Details on the complete setup can be found in recent publications..$^{54,55}$ The patterns were measured between 5 and $39^{\circ} 2 \theta$ with an increment of $0.07^{\circ}$ and time per step of $20.5 \mathrm{~s}$. Multiple scans were accumulated to improve the signalto-noise ratio only if the patterns were identical. Rietveld Quantitative Phase Analysis (Rietveld QPA) was performed on the measured diffractograms using Bruker TOPAS v5 software. Details of the Rietveld refinement procedure are given in Appendix D.

$\mathrm{Co}-\mathrm{S} / \mathrm{SiO}_{2}$ was diluted with 2 mass equivalents $\mathrm{SiO}_{2}$ (Aerosil OX 50) to obtain a similar overall cobalt loading as $\mathrm{Co}-\mathrm{D} / \mathrm{SiO}_{2}$. Typically, $7 \mathrm{mg}$ sample in the sieve fraction $75-150 \mu \mathrm{m}$ was loaded in the capillary and fixed with quartz wool at either end, resulting in a catalyst bed length of $\sim 20 \mathrm{~mm}$. The capillary was placed in the setup and the catalysts were reduced in situ at $350{ }^{\circ} \mathrm{C}$ for $8 \mathrm{~h}\left(1{ }^{\circ} \mathrm{C} \cdot \mathrm{min}^{-1}\right)$ in a $1.2 \mathrm{~mL} \cdot \mathrm{min}^{-1}$ flow of $25 \mathrm{vol} \% \mathrm{H}_{2}$ in He at 1 bar. After reduction, the temperature was lowered to $180{ }^{\circ} \mathrm{C}$ with $3{ }^{\circ} \mathrm{C} \cdot \mathrm{min}^{-1}$ and at this temperature, a $3.0 \mathrm{~mL} \cdot \mathrm{min}^{-1}$ flow of synthesis gas $\mathrm{H}_{2} / \mathrm{CO}=2(\mathrm{v} / \mathrm{v})$ was introduced. After $10 \mathrm{~min}$ stabilization, the back-pressure regulator was set to 10.6 bar. Only after the set pressure was reached and stable, the temperature was increased to $220^{\circ} \mathrm{C}$ with $1{ }^{\circ} \mathrm{C} \cdot \mathrm{min}^{-1}$, which was defined as TOS $=0 \mathrm{~h}$. In situ XRD patterns were recorded both during reduction and FT.

\subsubsection{Fischer-Tropsch synthesis}

The Fischer-Tropsch synthesis performance of the catalysts was investigated using a Flowrence (Avantium) 16 parallel reactor setup. $80 \mathrm{mg} \mathrm{Co}-\mathrm{D} / \mathrm{SiO}_{2}$ and $12 \mathrm{mg} \mathrm{Co}-\mathrm{S} / \mathrm{SiO}_{2}$ were first diluted with $100 \mathrm{mg} \mathrm{SiC} \mathrm{(212-425} \mathrm{\mu m)} \mathrm{and} \mathrm{then} \mathrm{loaded} \mathrm{into} \mathrm{stainless} \mathrm{steel} \mathrm{plug-}$ flow reactors of 2.6 and $2.0 \mathrm{~mm}$ (ID), respectively. The samples were dried for $2 \mathrm{~h}$ in an $\mathrm{He}$ flow and reduced in situ in a $25 \mathrm{vol}^{\%} \mathrm{H}_{2}$ in He flow at $350^{\circ} \mathrm{C}$ for $8 \mathrm{~h}$ after a heating ramp of $1{ }^{\circ} \mathrm{C} \cdot \mathrm{min}^{-1}$. After reduction, the temperature was lowered to $180{ }^{\circ} \mathrm{C}$ with $3{ }^{\circ} \mathrm{C} \cdot \mathrm{min}^{-1}$ and the reactors were pressurized to 20 bar in an $\mathrm{H}_{2}$ flow. Then, the synthesis gas feed was introduced with $\mathrm{H}_{2} / \mathrm{CO}=2 \mathrm{v} / \mathrm{v}$ and $5 \mathrm{vol}^{\%} \mathrm{He}$ as internal standard and after $1 \mathrm{~h}$ stabilization, the reactors were heated to $220{ }^{\circ} \mathrm{C}$ with $1{ }^{\circ} \mathrm{C} \cdot \mathrm{min}^{-1}$, which was defined as the starting point for FT. After the run, the catalysts were treated in $\mathrm{H}_{2}$ at $200{ }^{\circ} \mathrm{C}$ for $10 \mathrm{~h}$ to remove carbonaceous products from the catalyst surface and cooled down further under He.

The reaction products were analyzed online using an Agilent 7890A GC with two separate channels. In the first channel, the hydrocarbon products were analyzed using an Agilent J\&W PoraBOND Q column in combination with an FID. In the second channel, the permanent gasses were separated on a ShinCarbon-ST column and analyzed using a TCD. The catalytic activity was given as $\mathrm{CO}$ conversion $\left(\mathrm{X}_{\mathrm{CO}}\right)$ cobalt-time yield $(\mathrm{CTY})$ and 
turnover frequency (TOF). The TOF was based on the CTY and the average metallic surface area of the spent catalyst, as determined by TEM. The selectivity (in \%c) to $\mathrm{C}_{1}-\mathrm{C}_{4}$ hydrocarbons was determined as $\mathrm{S}_{\mathrm{C} 1-\mathrm{C} 4}=100 \cdot \mathrm{F}_{\mathrm{C} n} \cdot \mathrm{n} \cdot\left(\mathrm{F}_{\mathrm{CO}, \mathrm{in}} \cdot \mathrm{X}_{\mathrm{CO}}\right)^{-1}$ with $\mathrm{n}$ representing the product's carbon number and $\mathrm{F}$ the flow of the hydrocarbon or $\mathrm{CO}$. The selectivity to $\mathrm{C}_{5+}$ hydrocarbons was calculated as $\mathrm{S}_{\mathrm{C} 5+}=100 \% \mathrm{c}^{-} \mathrm{S}_{\mathrm{C1}-4}$.

\subsection{Results and discussion}

\subsubsection{Cobalt nanocrystal synthesis}

Cobalt nanocrystals (NC) were synthesized by a hot-injection synthesis using oleic acid and octadecylamine as ligands with the aim to synthesize disk-shaped NC and the results were analyzed by TEM (Figure 5.1). Both disk- and spherical-shaped $\mathrm{CoO}-\mathrm{NC}$ were obtained from a single synthesis with the ratio between the two varying strongly with the reaction conditions, which made separation necessary, in line with literature..$^{10}$ Enrichment of the disk-shaped over spherical NC was realized by a magnetic field, using the difference in the magnetic properties between the different NC. In our case, the large magnetic moment of the spheres resulted in faster precipitation from the solution compared to the disks under the weak magnetic field of the stirring plate (Appendix D, Figure D1). This led to enhanced concentrations of disks in the top layer of the reaction mixture (Figure 5.1a-e). After magnetic separation, the work-up procedure also affected the disk-to-sphere ratio, as the disk yield decreased with more precipitation-redispersion cycles (Figure 5.1a-c). This indicates that spheres were preferentially precipitated during centrifugation or alternatively, that the fraction of disks already varied in the original reaction mixture of different batches.

The elongated, rod-like particles (mainly visible in Figure 5.1a) were identified as stacks of disk-shaped particles orientated side to side. From these particles, the average dimensions of the disks were determined (Appendix D, Figure D2). The average diameter of the disks was $15.5 \pm 3.5 \mathrm{~nm}$ and the thickness $4.2 \pm 0.6 \mathrm{~nm}$, leading to an aspect ratio (height/diameter) of 0.27 . As a comparison, all-spherical $\mathrm{CoO}-\mathrm{NC}$ of $8.3 \pm 1.1 \mathrm{~nm}$ were synthesized using only oleic acid as ligand, as described before ${ }^{49}$ (Figure 5.1f).

A batch containing disk-shaped NC after three precipitation-redispersion cycles was analyzed in more detail (Figure 5.1c). The NC in the TEM image were semi-automatically divided into two groups (Figure 5.1e) based on their contrast, which is a measure for their thickness, in relation to their size and morphology. The large, low-contrast NC were (truncated) hexagonally-shaped and the smaller, high-contrast NC were spherical. To examine the 3D shape of these NC, the stage was tilted $50^{\circ}$ inside the TEM (Figures $1 \mathrm{~d}$ and A3). Analysis of $100 \mathrm{NC}$ from both groups (Appendix D, Figure D4) revealed that the size of the particles was $15.4 \mathrm{~nm}$ at $0{ }^{\circ}$ and $11.9 \mathrm{~nm}$ at $50^{\circ}$ in the case of the low-contrast particles, proving that these $\mathrm{NC}$ were indeed smaller in the $z$ direction as expected for disk-shaped NC. When considering an aspect ratio of 0.27 , the $2 \mathrm{D}$ projection length at $50^{\circ}$ should be $13.1 \mathrm{~nm}$ (Appendix D, Figure D5), which matches the experimental value quite well. On the other hand, the smaller, high-contrast particles were $10.7 \mathrm{~nm}$ at $0^{\circ}$ and $10.6 \mathrm{~nm}$ at $50^{\circ}$, showing that their diameter was the same in all directions and that these $\mathrm{NC}$ were indeed 
spherical. Because the shapes of the $\mathrm{CoO}-\mathrm{NC}$ could be accurately distinguished, the numerical disk yield could be determined and was $53 \%$ for the batch after three precipitation-redispersion cycles.

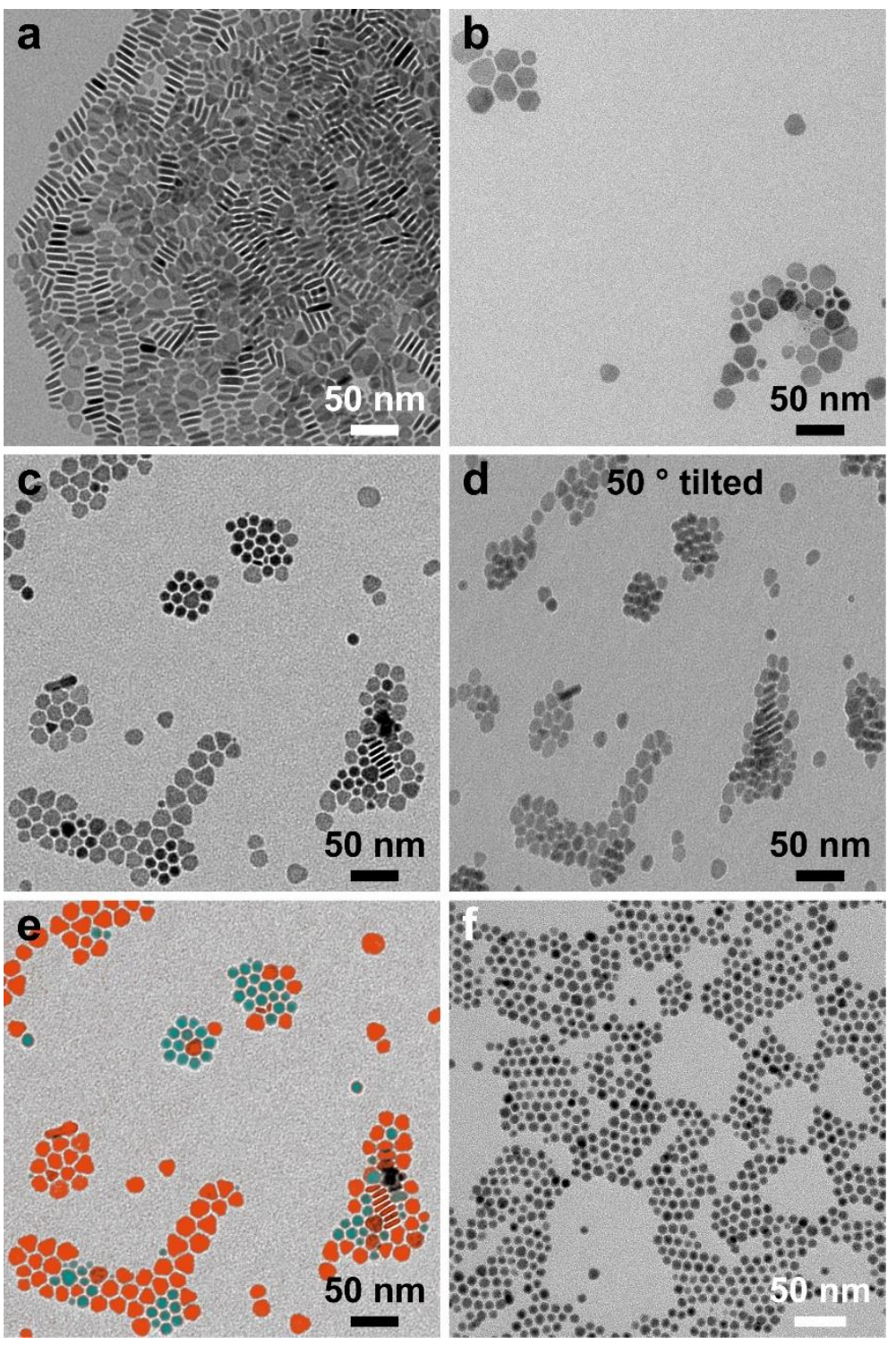

Figure 5.1. TEM images of several batches of mixed spheres/disks $\mathrm{CoO}-\mathrm{NC}$ after different washing procedures followed by dispersion and drying on the carbon foil a-e. The washing procedures consisted of $\mathbf{a}$ one precipitation-redispersion cycle, $\mathbf{b}$ two precipitation-redispersion cycles and $\mathbf{c}-$ e three precipitation-redispersion cycles. $\mathbf{c}$-e Images of the same location with $\mathbf{d}$ showing the sample after tilting by $50^{\circ}$ and e with an overlay highlighting the particles that were identified as disks (red) and spheres (aqua). f Spherical CoO-NC synthesized using only oleic acid as ligand.

Disk-shaped Co-NC are a metastable phase, ${ }^{8,56}$ making the synthesis results highly susceptible to small variations in the procedure. Therefore, optimizing the reaction conditions was essential to obtain a high fraction of anisotropic particles. Important synthesis parameters included the speed of magnetic stirring and the speed of precursor injection, as faster stirring or slower injection through a thinner needle led to enhanced formation of 10-30 nm spherical NC and a lower yield of disk-shaped NC. Furthermore, 
higher disk yields were obtained with batches of $\mathrm{Co}_{2}(\mathrm{CO})_{8}$ that had been aged $40 \mathrm{~min}$ in the glovebox prior to use in synthesis. These phenomena have not been reported before for this synthesis method and illustrate the delicate nature of this approach.

The crystal structure of the as-synthesized Co-NC before oxidation was analyzed using a combined cryo- and high-resolution-TEM approach (Figure 5.2). A Co-NC suspension was vitrified shortly after NC synthesis and transported and loaded in liquid $\mathrm{N}_{2}$ to avoid oxidation of the Co-NC. The vitrification protected the sample from oxidation and HR-TEM was possible because there was little to no solvent present in the TEM sample. Electron diffraction of the area in Figure 5.2a resulted in the diffraction pattern in the inset of Figure 5.2a and was radially averaged to give Figure 5.2b. The main peak at $d=2.06 \AA$ could not be unambiguously assigned to one crystal structure, as this lattice spacing is present in all three metallic cobalt phases (Appendix D, Figure D6). The diffraction peak at $\mathrm{d}=2.19 \AA$, however, only matched hcp cobalt. Furthermore, the peaks at d-values of 1.26 and $2.19 \AA$ could be ascribed to $\{11 \overline{20}\}$ and $\{10 \overline{0}\}$ of hcp cobalt, respectively, which were more intense than expected. Considering that $\{11 \overline{20}\}$ and $\{10 \overline{10}\}$ have periodicity in the $x, y$ direction and not in the $z$ direction, this proved that the hcp cobalt was present in anisotropic, diskshaped NC and that the short $z$ axis is along the [0001] direction, in line with literature. ${ }^{10}$ Based on the intensity ratios of the hcp Co phase, it is likely that the peak at $d=2.06 \AA$ has another dominant contribution either from fcc or $\varepsilon$-cobalt.
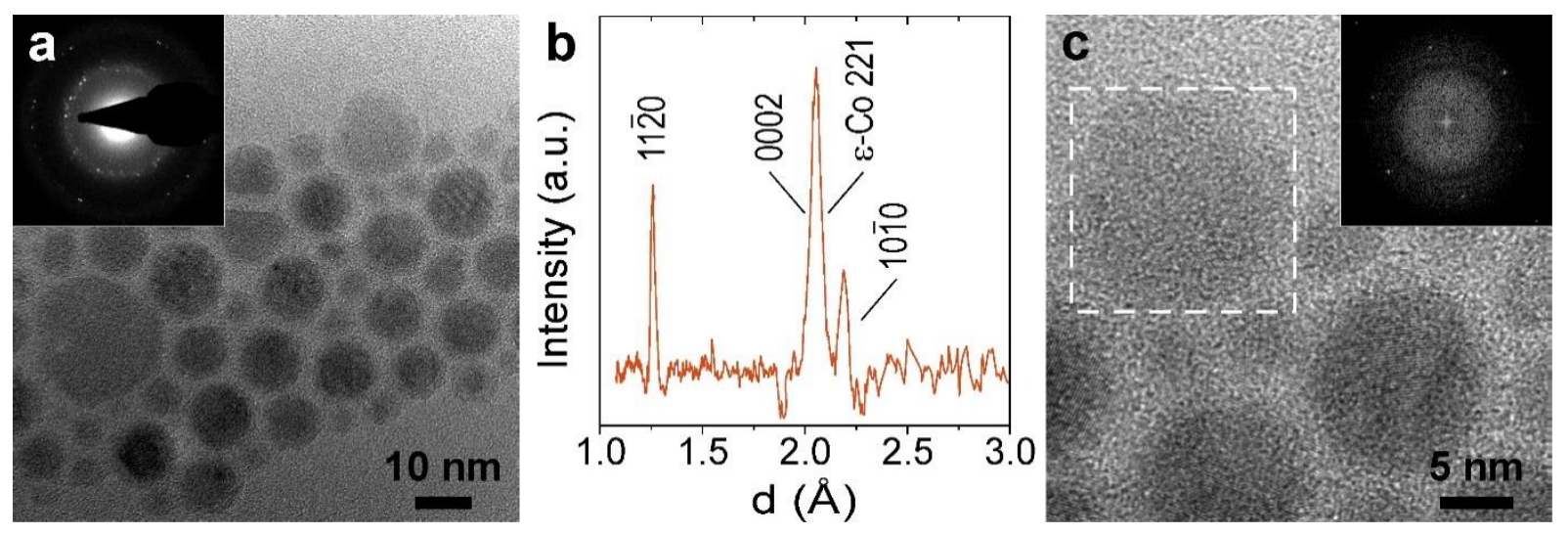

Figure 5.2. High-resolution TEM under cryogenic conditions of as-synthesized disk-shaped and spherical Co-NC with little to no solvent present. a Overview of several clustered Co-NC with the selected area electron diffraction pattern in the inset. $\mathbf{b}$ Intensity profile obtained by radially averaging and baseline correcting the SAED pattern. $\mathbf{c}$ Several Co-NC at higher magnification. The framed area contained a $15 \mathrm{~nm}$, low contrast NC that was classified as a disk; the inset gives the Fourier transform of the framed area.

Further information on the crystal structure of the NC before oxidation was obtained by investigating an individual $\mathrm{NC}$ in more detail with HR-TEM on the same cryogenic sample. The Co-NC in the framed area in Figure 5.2c was identified as a disk-shaped NC because of its low contrast and diameter of $15 \mathrm{~nm}$. Lattice fringes of this particle were imaged and the Fourier transform revealed hexagonal symmetry of the crystal lattice, indicative of hcp cobalt. The determined lattice spacings were 2.28 and $1.29 \AA$, which were 
again matched to $\{11 \overline{20}\}$ and $\{10 \overline{10}\}$ of hcp Co. Additionally, the other prominent particle of $11 \mathrm{~nm}$ with higher contrast was identified as a spherical $\mathrm{NC}$ and did not show hexagonal symmetry. Its lattice spacings matched $\varepsilon$-cobalt best (Appendix D, Figure D7), indicating that the other crystal structure observed by electron diffraction was most likely $\varepsilon$-cobalt, which is consistent with literature. ${ }^{10}$ It should be emphasized that this concerned the crystal structure of the Co-NC before oxidation and that it is uncertain what crystal structure would evolve after low-temperature oxidation, in situ reduction and catalysis.

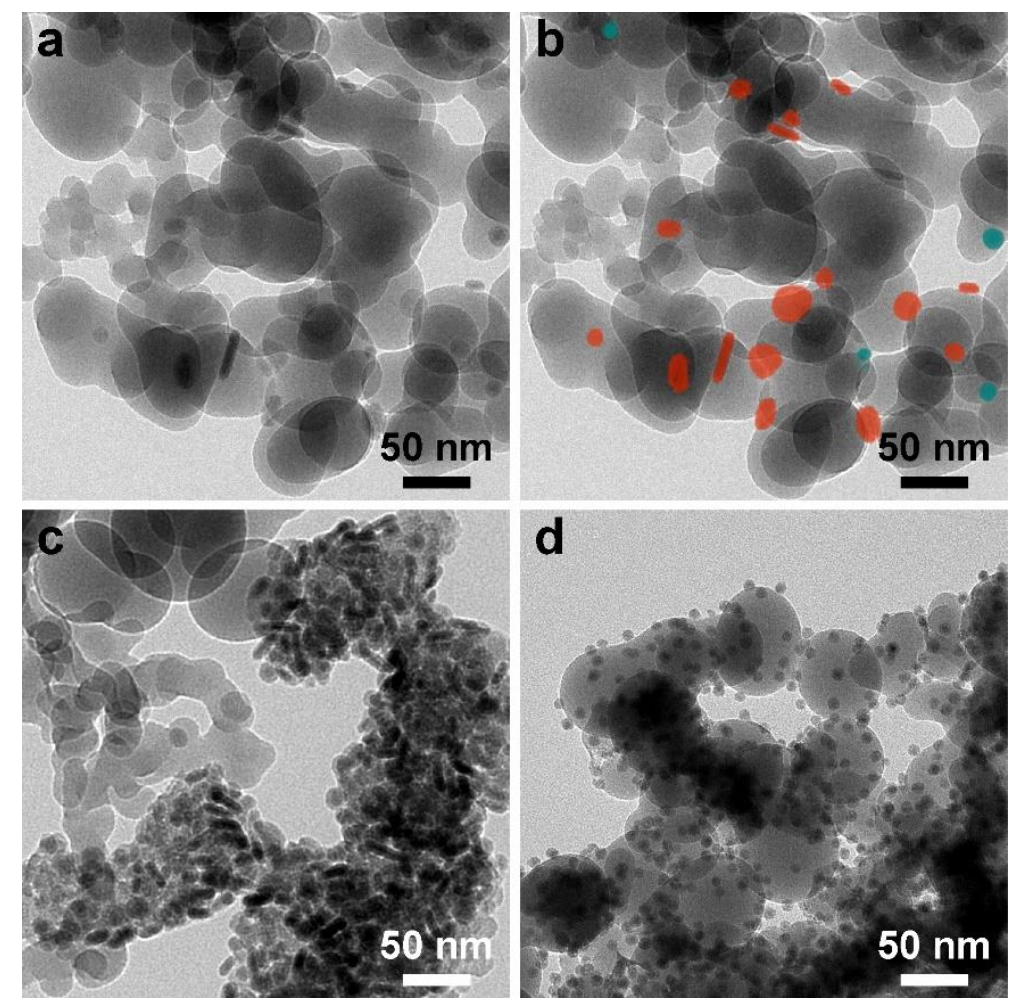

Figure 5.3. TEM images of pristine samples of $\mathrm{CoO}-\mathrm{NC}$ attached to $\mathrm{SiO}_{2}$. a An area of $\mathrm{Co}-\mathrm{D} / \mathrm{SiO}_{2}$ with an uniform distribution of $\mathrm{CoO}-\mathrm{NC}$ and $\mathbf{b}$ the same location with an overlay highlighting the $\mathrm{NC}$ that were identified as disks (red) and spheres (aqua). $\mathbf{c}$ An area of $\mathrm{Co}-\mathrm{D} / \mathrm{SiO}_{2}$ where the $\mathrm{CoO}-\mathrm{NC}$ were clustered. $\mathbf{d ~ C o}-\mathrm{S} / \mathrm{SiO}_{2}$ with an uniform $\mathrm{CoO}-\mathrm{NC}$ distribution.

\subsubsection{Cobalt nanocrystal attachment}

The CoO-NC after two precipitation-redispersion cycles were attached to low surface area $\mathrm{SiO}_{2}\left(50 \mathrm{~m}^{2} \cdot \mathrm{g}^{-1}\right)$ using a method that avoids the use of high temperatures to affect the NC shape as little as possible. This particular type of $\mathrm{SiO}_{2}$ was selected, because it had yielded very stable Co-NC based catalysts under FT conditions before. ${ }^{49}$ The sample containing mainly disk-shaped $\mathrm{NC}$ was designated as $\mathrm{Co}-\mathrm{D} / \mathrm{SiO}_{2}$. Two washing steps after $\mathrm{NC}$ synthesis were the optimal compromise between uniform attachment of the $\mathrm{CoO}-\mathrm{NC}$ with lower ligand concentration on one hand (Appendix D, Figure D8) and progressively lower $\mathrm{NC}$ yields on the other. Based on ICP-OES, a cobalt loading of $1.4 \mathrm{wt} \%$ was obtained using this attachment method. The $\mathrm{Co}-\mathrm{D} / \mathrm{SiO}_{2}$ sample was analyzed by TEM (Figure $5.3 \mathrm{a}-\mathrm{c}$ ). The NC were largely uniformly distributed over the support, although some areas with clustered disk-shaped and spherical NC were encountered as well (Figure 5.3c). Despite 
this occasional clustering of NC, NC were largely well dispersed on the support, making the catalyst useful for further investigations.
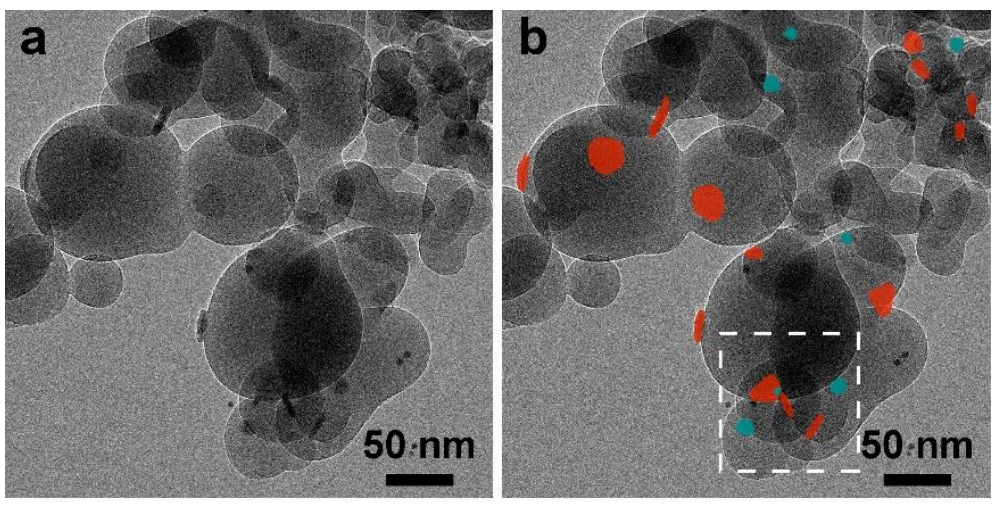

C

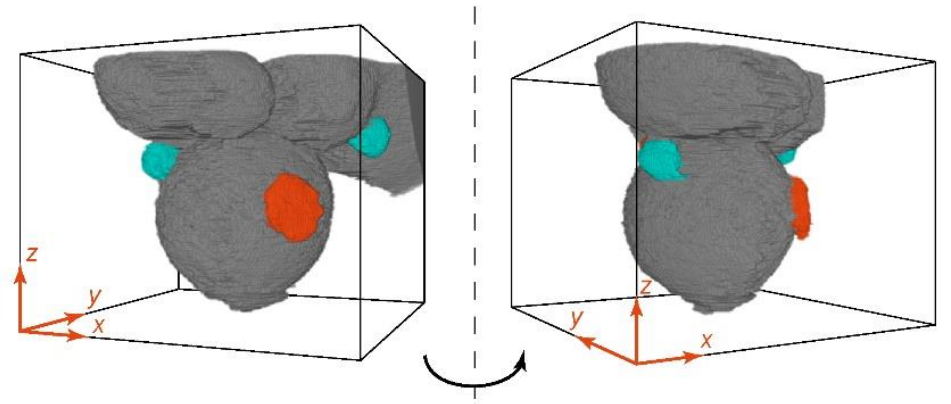

Figure 5.4. Electron tomography results of the pristine $\mathrm{Co}-\mathrm{D} / \mathrm{SiO}_{2}$ sample, consisting of a mixture of disk-shaped and spherical $\mathrm{CoO}-\mathrm{NC}$ attached to $\mathrm{SiO}_{2}$. a TEM image of $\mathrm{Co}-\mathrm{D} / \mathrm{SiO}_{2}$ at $0{ }^{\circ}$ tilt angle. The small $(\sim 5 \mathrm{~nm})$, high-contrast particles are gold fudicial markers on the TEM grid. $\mathbf{b}$ The same TEM image with an overlay highlighting the NC that were identified as disks (red) and spheres (aqua). This location was reconstructed and the framed area was segmented. c Volume-rendered 3D reconstruction of the framed area in (b), shown at two different angles obtained by rotation along the $z$ axis (dashed line). The $z$ axis also indicates the direction of the projection in the original TEM image a,b. The $\mathrm{SiO}_{2}$ support is depicted in grey, disk-shaped $\mathrm{CoO}-\mathrm{NC}$ in red and spherical CoO-NC in aqua. An animation showing a $360^{\circ}$ rotation of this segmented sample together with the tilt series and the full reconstruction file can be found online as supporting movies of the original article.

It was challenging to distinguish the disk-shaped $\mathrm{NC}$ from the $\mathrm{SiO}_{2}$ support because of the low contrast of the $\mathrm{CoO}-\mathrm{NC}$ associated with their limited thickness when orientated perpendicular to the electron beam. However, based on a combination of contrast, size and morphology, it was possible to identify the $\mathrm{CoO}-\mathrm{NC}$ and discriminate between disks and spheres (Figure 5.3a,b). After analysis of $203 \mathrm{NC}, 66 \%$ of the NC were classified as disks and $34 \%$ as spheres. In addition, a reference catalyst containing exclusively spherical CoO-NC was prepared by attaching the $8.3 \mathrm{~nm} \mathrm{CoO}-\mathrm{NC}$ to the same type of low surface area $\mathrm{SiO}_{2}$ (Figure 5.3d). This catalyst was designated as $\mathrm{Co}-\mathrm{S} / \mathrm{SiO}_{2}$. The obtained cobalt loading was $9.6 \mathrm{wt} \%$ based on ICP-OES. Furthermore, the $\mathrm{CoO}-\mathrm{NC}$ were uniformly distributed over the support and no areas containing clustered $\mathrm{NC}$ were observed. This catalyst was described in more detail in an earlier publication. ${ }^{49}$ 
Electron tomography of $\mathrm{Co}-\mathrm{D} / \mathrm{SiO}_{2}$ was performed to investigate the morphology of the NC on the support in more detail (Figure 5.4). The disks could be clearly distinguished from the spheres using the tilt series, because only the projected aspect ratio of the disks varied with the tilting angle. The accuracy of the method applied before to classify the NC as disks or spheres based on size, morphology and contrast was confirmed by tomography and the obtained results for this limited sample size (only $26 \mathrm{NC}$ in field of view) matched the earlier observation of $\sim 65 \%$ of the NC present as disks. Furthermore, a 3D representation of a selected part of the sample was obtained through reconstruction and segmentation of the tilt series (Figure 5.4c). From this representation, the morphology of $\mathrm{NC}$ can be clearly inferred as well as their interaction with the support. It showed that the disk-shaped NC typically attached to $\mathrm{SiO}_{2}$ with one base plane.

\subsubsection{Fischer-Tropsch synthesis}

The FT performance of the catalysts was evaluated over $100 \mathrm{~h}$ on stream at similar CO conversion levels (Figure 5.5, Table 5.1). The cobalt-weight-based activity (cobalt-time yield, CTY) against TOS is shown in Figure 5.5b. $\mathrm{Co}-\mathrm{S} / \mathrm{SiO}_{2}$ was twice as active per unit weight of cobalt as $\mathrm{Co}-\mathrm{D} / \mathrm{SiO}_{2}$. Because of the different particle sizes and shapes, the activity was compared as cobalt-surface-based activity (turnover frequency, TOF). The metallic surface area necessary for the TOF was calculated from the NC sizes in the spent catalyst, which will be discussed later (Table 5.1). The TOF of $\mathrm{Co}-\mathrm{D} / \mathrm{SiO}_{2}$ was $49 \cdot 10^{-3} \mathrm{~s}^{-1}$ and the TOF of $\mathrm{Co}-\mathrm{S} / \mathrm{SiO}_{2}$ was $86 \cdot 10^{-3} \mathrm{~s}^{-1}$, the former value being in line with other work on $\mathrm{Co} / \mathrm{SiO}_{2}$ using conventional catalyst preparation techniques such as impregnation. ${ }^{57}$ However, the TOF of $\mathrm{Co}-\mathrm{S} / \mathrm{SiO}_{2}$ was remarkably high, possibly pointing to a higher fraction of more active hcp $\mathrm{Co}^{22-26}$ resulting from the NC-based synthesis compared to conventional synthesis methods.

Table 5.1. Fischer-Tropsch synthesis results for $\mathrm{Co}-\mathrm{D} / \mathrm{SiO}_{2}$ and $\mathrm{Co}-\mathrm{S} / \mathrm{SiO}_{2}$ reported at similar $\mathrm{CO}$ conversions and averaged between $90-100 \mathrm{~h}$ on stream at $20 \mathrm{bar}, 220{ }^{\circ} \mathrm{C}$ and $\mathrm{H}_{2} / \mathrm{CO}=2 \mathrm{v} / \mathrm{v}$.

\begin{tabular}{llllllllll}
\hline Sample & $\begin{array}{l}\text { GHSV } \\
\left(\mathbf{h}^{-1}\right)\end{array}$ & $\begin{array}{l}\mathrm{X}_{\mathrm{CO}} \\
(\%)\end{array}$ & $\begin{array}{l}\mathrm{CTY} \\
\mathbf{a}\end{array}$ & $\begin{array}{l}\mathrm{TOF}_{\text {app }} \\
\left(\mathbf{1 0}^{-3} \mathbf{s}^{-1}\right)^{\mathrm{b}}\end{array}$ & $\begin{array}{l}\mathrm{S}_{\mathrm{C} 1} \\
(\%)\end{array}$ & $\begin{array}{l}\mathrm{S}_{\mathrm{C} 2 \mathrm{C} 4} \\
(\%)\end{array}$ & $\begin{array}{l}\mathrm{S}_{\mathrm{C} 5+} \\
(\%)\end{array}$ & $\alpha$ & $\begin{array}{l}\mathrm{Co} \mathrm{SA}_{\text {spent }} \\
\left(\mathbf{m}^{2} \cdot \mathrm{g}_{\mathrm{Co}}{ }^{-1}\right)\end{array}$ \\
\hline $\mathrm{Co}-\mathrm{D} / \mathrm{SiO}_{2}$ & 1560 & 8.9 & 6.9 & 49 & 16 & 8.6 & 75 & 0.78 & 56 \\
$\mathrm{Co}-\mathrm{S} / \mathrm{SiO}_{2}$ & 39900 & 8.1 & 15 & 86 & 13 & 9 & 78 & 0.76 & 71 \\
\hline
\end{tabular}

a in $10^{-5} \mathrm{~mol}_{\mathrm{CO}} \cdot \mathrm{g}_{\mathrm{Co}}{ }^{-1} \cdot \mathrm{s}^{-1}$

${ }^{\mathrm{b}}$ based on the CTY and the metallic Co surface area of the spent catalytst as derived by TEM.

The selectivities towards $\mathrm{C}_{5+}-$ as well as $\mathrm{C}_{1}-$ and $\mathrm{C}_{2}-\mathrm{C}_{4}$ - products were similar (Table 5.1) and overall $\sim 5 \%$ lower than on conventionally prepared $\mathrm{Co} / \mathrm{SiO}_{2}$ catalysts. ${ }^{57}$ Consequently, the ASF distributions and derived chain growth probabilities $(\alpha)$ were comparable as well. Plots of the $\mathrm{C}_{5_{+}}-$selectivity as a function of TOS and ASF distributions are included as Appendix D, Figure D9. The effect of crystal structure on selectivity is still under debate, as some reports indicated no difference between hcp and fcc Co, ${ }^{22,27}$ while others observed higher $\mathrm{C}_{5+}$-selectivity on hcp $\mathrm{Co}^{24,58}$ However, in our case with anticipated enhanced (0001) for $\mathrm{Co}-\mathrm{D} / \mathrm{SiO}_{2}$, major differences in selectivity would be unexpected considering that the inactive surfaces, e.g. (0001), contribute only marginally to the overall product 
spectrum. Thus, changing the NC shape from spheres to disks did not influence the selectivity of the catalyst. Overall, the main difference in performance was the factor 2 lower intrinsic activity of the disk-containing $\mathrm{Co}-\mathrm{D} / \mathrm{SiO}_{2}$ sample, which could have several reasons. First, the activity could be lower as a result of the shape and thus the exposed facets, which would match the theoretical and experimental finding that hcp (0001) is less active than higher-index hep or fcc facets. ${ }^{25,32}$ Second, residual ligands could still be present in the case of $\mathrm{Co}-\mathrm{D} / \mathrm{SiO}_{2}$ and block part of the cobalt surface. Third, the surface area of Co$\mathrm{D} / \mathrm{SiO}_{2}$ could be lower than expected if the degree of reduction is low or if there is substantial NC growth, for example in the areas where clustering of NC was observed (Figure 5.3c). These potential explanations were investigated in more detail and are described below.
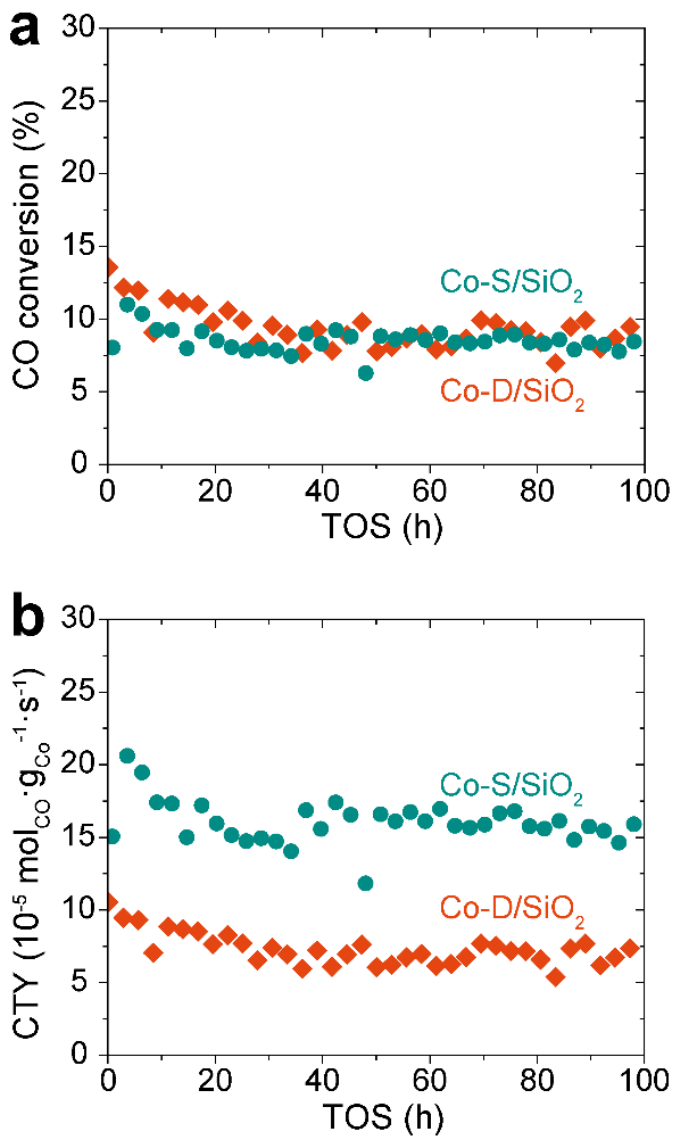

Figure 5.5. Fischer-Tropsch synthesis results of $\mathrm{Co}-\mathrm{D} / \mathrm{SiO}_{2}$ and $\mathrm{Co}-\mathrm{S} / \mathrm{SiO}_{2}$ at $20 \mathrm{bar}, 220{ }^{\circ} \mathrm{C}$ and $\mathrm{H}_{2} / \mathrm{CO}=2 \mathrm{v} / \mathrm{v}$. a CO conversion and $\mathbf{b}$ cobalt-time yield as a function of time-on-stream.

\subsubsection{Characterization of spent catalysts}

The structure of the spent catalysts was analyzed after FT and subsequent passivation using TEM (Figure 5.6). The sizes and shapes of 160 cobalt nanocrystals in $\mathrm{Co}-\mathrm{D} / \mathrm{SiO}_{2}$ were quantified (Figure 5.6a,b), showing numerically $62 \%$ disk-shaped NC with $\mathrm{d}=14.5 \mathrm{~nm}$ and $38 \%$ spherical $\mathrm{NC}$ with $\mathrm{d}=10.2 \mathrm{~nm}$. The aspect ratio of the disks could not be reliably determined for this sample and was assumed to have remained unchanged. These results 
were very similar to those of the pristine catalyst and the disk shape was thus remarkably stable under FT conditions. Furthermore, a more clustered part of the catalyst was also observed where some particle growth had occurred (Figure 5.6c). This area probably originated from an area of clustered $\mathrm{CoO}-\mathrm{NC}$ in the pristine catalyst. The size of the NC in $\mathrm{Co}-\mathrm{S} / \mathrm{SiO}_{2}$ was unchanged as well with spherical particles of $8.7 \mathrm{~nm}$ on average (Figure 5.6d). The stability of the Co-NC in terms of both size and shape under reaction conditions meant that catalytic performance might be related to the disk shape.
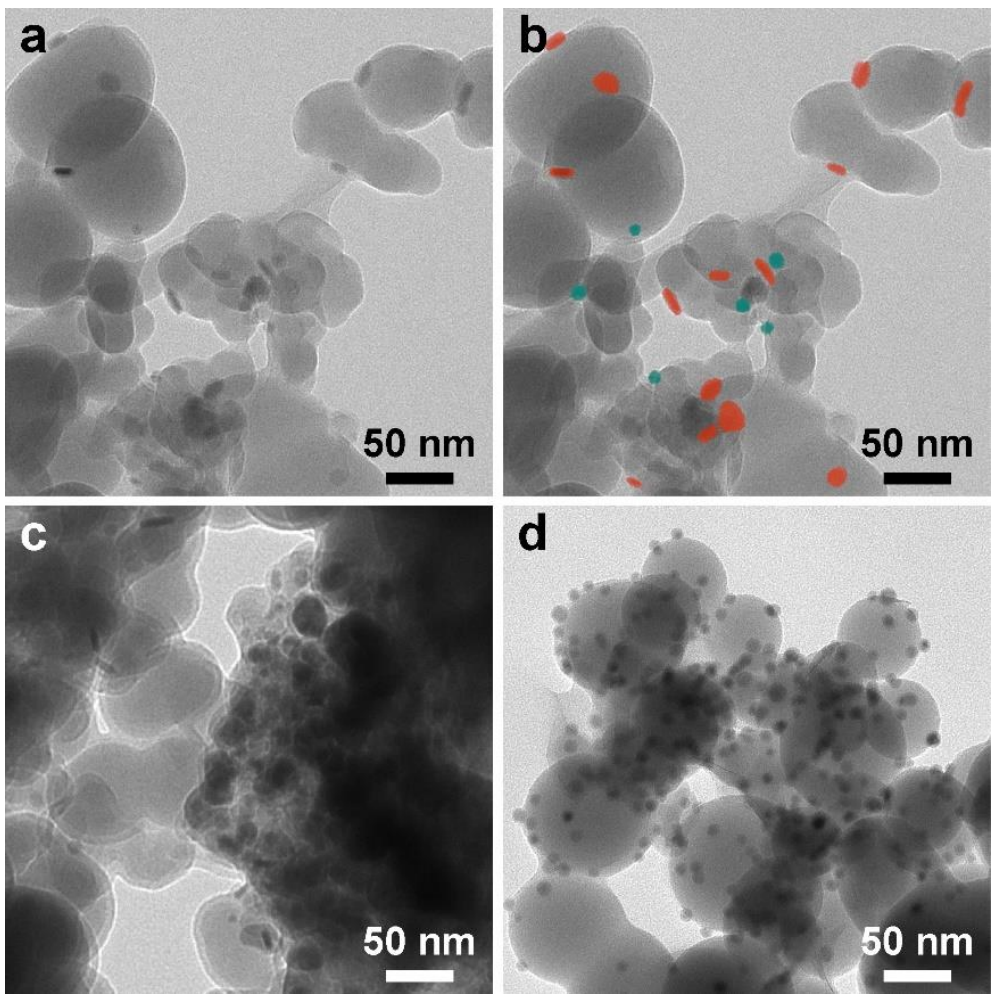

Figure 5.6. TEM images of $\mathrm{Co} / \mathrm{SiO}_{2}$ samples after $>100 \mathrm{~h}$ of Fischer-Tropsch synthesis and passivation. a An area of $\mathrm{Co}-\mathrm{D} / \mathrm{SiO}_{2}$ with an uniform distribution of $\mathrm{NC}$ and $\mathbf{b}$ the same location with an overlay highlighting the $\mathrm{NC}$ that were identified as disks (red) and spheres (aqua). c An area of $\mathrm{Co}-\mathrm{D} / \mathrm{SiO}_{2}$ where the $\mathrm{NC}$ were clustered. $\mathbf{d} \mathrm{Co}-\mathrm{S} / \mathrm{SiO}_{2}$ with a homogeneous NC distribution.

Two types of ligands were used to synthesize disk-shaped Co-NC (oleic acid and octadecylamine) while spherical Co-NC were synthesized using only oleic acid. For oleic acid, efficient ligand removal has been shown, ${ }^{7}$ but could be different for linear amines. We assessed the influence of the octadecylamine ligand in FT by investigating the amount of surface nitrogen species using XPS on the catalysts as-prepared and after $100 \mathrm{~h}$ FT followed by passivation. Low concentrations of $\mathrm{N}$ were detected on both as-prepared catalysts and on bare $\mathrm{SiO}_{2}$ (Figure 5.7a, complete survey scans in Appendix D, Figure D10) The N-to-Co atom ratio was 4 times higher on $\mathrm{Co}-\mathrm{D} / \mathrm{SiO}_{2}(0.07)$ than on $\mathrm{Co}-\mathrm{S} / \mathrm{SiO}_{2}(0.02)$. After FT and passivation, $\mathrm{Co}-\mathrm{D} / \mathrm{SiO}_{2}$ was partially covered in carbon and only an indication of $\mathrm{N}$ was observed. Spent $\mathrm{Co}-\mathrm{S} / \mathrm{SiO}_{2}$ was too covered in carbon to yield useful data on Co and $\mathrm{N}$ contents. The $\mathrm{N} / \mathrm{Co}$ atom ratio of spent $\mathrm{Co}-\mathrm{D} / \mathrm{SiO}_{2}$ seemed similar to that of as-prepared $\mathrm{Co}-\mathrm{S} / \mathrm{SiO}_{2}$, so excess $\mathrm{N}$-species in $\mathrm{Co}-\mathrm{D} / \mathrm{SiO}_{2}$ from the $\mathrm{NC}$ synthesis procedure were 
removed during reduction/FT. Harmel et al. ${ }^{46}$ quantified that the content of the ligand hexadecylamine decreased from $8 \mathrm{wt} \%$ to $3 \mathrm{wt} \%$ during reduction under $\mathrm{H}_{2}$ at $270{ }^{\circ} \mathrm{C}$, which is substantially lower than the reduction temperature of $350^{\circ} \mathrm{C}$ applied here. Therefore, it seemed that our reduction procedure was sufficient to activate the catalyst and minimize the effect of remaining $\mathrm{N}$-species.

To get more insight into the structure of the catalysts under FT conditions, in situ XRD was performed on both catalysts at 10 bar, $220{ }^{\circ} \mathrm{C}$ and an $\mathrm{H}_{2} / \mathrm{CO}$ ratio of $2 \mathrm{v} / \mathrm{v}$ (Figure $5.7 \mathrm{~b}$, A11). To obtain satisfying Rietveld refinement results, it was necessary to allow preferred orientations of hcp Co crystallites, similar to what has been reported before. ${ }^{55} \mathrm{We}$ ascribe this to polymorphism of $\mathrm{Co}$ in the $\mathrm{Co}-\mathrm{NC} / \mathrm{SiO}_{2}$ samples, since XRD analysis of metallic cobalt is complicated by the propensity of cobalt to include stacking faults. ${ }^{22,59}$ Our analysis method did not quantify this intergrowth structure, but accommodated much of it in the hcp contribution by increasing the intensity of the (0002) peak. The fraction of hcp Co could therefore be overestimated. However, both samples were refined using the same method and given the similarity of the results for both samples, it appears that polymorphism, if present, took place to the same extent in both samples.

The crystalline cobalt phase in both catalysts consisted of approximately $65 \%$ hcp Co. The fec $\mathrm{Co}$ and $\mathrm{CoO}$ compositions were $21 \%$ fcc $\mathrm{Co}$ and $12 \% \mathrm{CoO}$ for $\mathrm{Co}-\mathrm{D} / \mathrm{SiO}_{2}$ and $32 \%$ fcc $\mathrm{Co}$ and $3 \% \mathrm{CoO}$ for $\mathrm{Co}-\mathrm{S} / \mathrm{SiO}_{2}$. These values are in line with results obtained by Andreev et al. ${ }^{60}$ using NMR on $\mathrm{Co} / \mathrm{SiC}$ at elevated temperatures under static $\mathrm{Ar}$ atmosphere and in reasonable agreement with Cats et al. ${ }^{54}$ who used operando XRD on $\mathrm{Co} / \mathrm{TiO}_{2}$ under FT conditions. In general, however, direct comparison of these results to literature is cumbersome, since the obtained Co phase strongly depends on particle size, metal precursor, support and activation procedure. ${ }^{61,62}$ Furthermore, the average crystallite sizes of metallic cobalt was 3-5 nm, significantly smaller than the particle dimensions obtained by TEM. This probably indicated that the Co-NC were polycrystalline, which could be related to the occurrence of stacking faults.

The degree of reduction of the crystalline phase was high with $88 \%$ for $\mathrm{Co}-\mathrm{D} / \mathrm{SiO}_{2}$ and $97 \%$ for $\mathrm{Co}-\mathrm{S} / \mathrm{SiO}_{2}$ and therefore excluded as a reason for substantial activity differences. Once in the reduced state, the XRD profiles remained unchanged for the duration of the experiment. The crystallites thus remained stable without sintering or restructuring during reduction and the first $18 \mathrm{~h}$ FT, in line with the TEM analyses. This also indicated that a significant loss of surface area in $\mathrm{Co}-\mathrm{D} / \mathrm{SiO}_{2}$ due to sintering of $\mathrm{NC}$ was unlikely. Furthermore, the hcp Co content in $\mathrm{Co}-\mathrm{D} / \mathrm{SiO}_{2}$ was similar to that in $\mathrm{Co}-\mathrm{S} / \mathrm{SiO}_{2}$, but nevertheless substantial in both samples. Finally, the average crystallite dimensions of 3$5 \mathrm{~nm}$ prevented any conclusions on potential preferred orientations and surface termination in disk-shaped NC with a diameter of $16 \mathrm{~nm}$ and $4 \mathrm{~nm}$ thickness. Overall, activity differences due to enhanced hcp Co content was excluded, but preferred crystallite orientations within the polycrystalline disk-shaped NC and with that enhanced hcp (0001) terminations were still possible. 

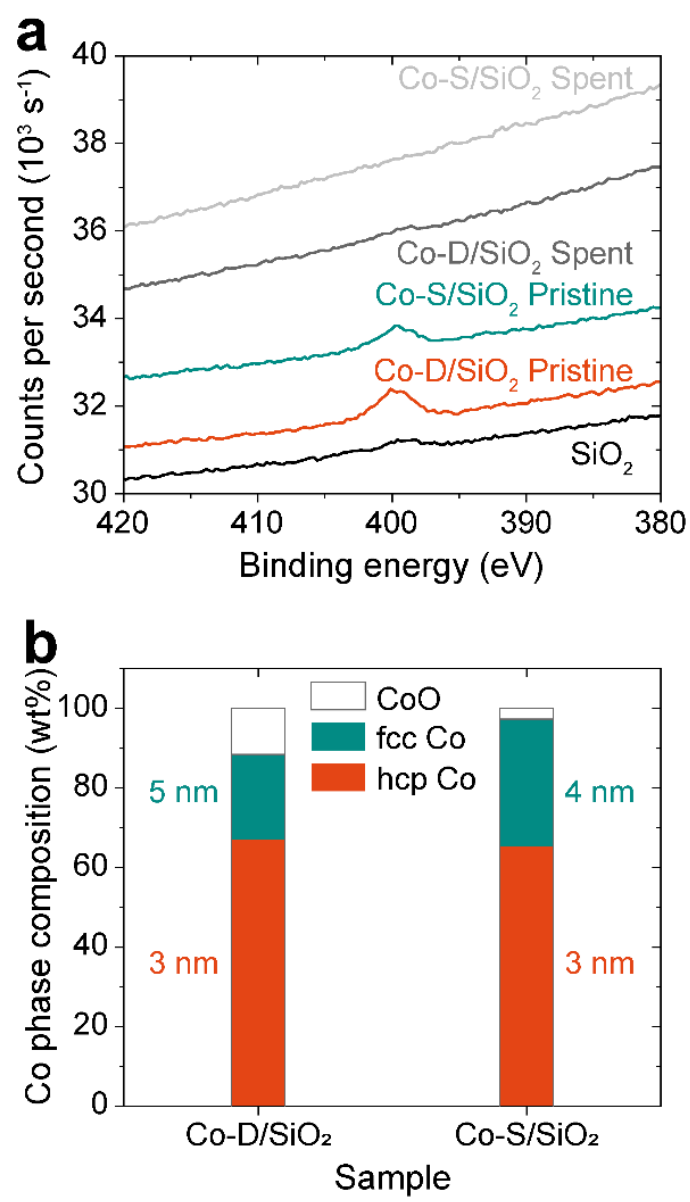

Figure 5.7. Nitrogen species on the catalysts before and after $100 \mathrm{~h} \mathrm{FT} \mathrm{followed} \mathrm{by} \mathrm{passivation,} \mathrm{and}$ cobalt crystal structure during FT. a N1s XPS signal around $400 \mathrm{eV}$ of the catalysts in the pristine and spent state and a bare $\mathrm{SiO}_{2}$ reference. The data was offset with $1500 \mathrm{CPS}$ increment at $380 \mathrm{eV}$ binding energy, except for $\mathrm{Co}-\mathrm{S} / \mathrm{SiO}_{2}$ spent (offset $-8000 \mathrm{CPS}$ ). $\mathrm{b}$ cobalt phase composition during FischerTropsch synthesis as determined using in situ XRD and Rietveld refinement. The average crystallite sizes per metallic Co phase are indicated next to the corresponding columns. The amount of CoO was too low to reliably determine a crystallite size. The diffractograms were obtained over the first $18 \mathrm{~h}$ on stream at $10 \mathrm{bar}, 220^{\circ} \mathrm{C}$ and $\mathrm{H}_{2} / \mathrm{CO}=2 \mathrm{v} / \mathrm{v}$ by accumulating six scans of $3 \mathrm{~h}$. No change in the diffraction patterns was observed over the course of the reaction.

Combining the obtained data on the ligands and NC size, shape and crystal structure of the catalysts under reaction conditions, the cobalt specific surface area and the fraction of the surface terminated by hcp (0001) were calculated (Figure 5.8). This served as an estimation of the structural difference between the $\mathrm{Co}-\mathrm{NC}$ disk-containing catalyst and the catalyst containing exclusively spherical NC. To do so, we assumed that the base planes of the disks were terminated by hcp (0001) and that the disks were attached to $\mathrm{SiO}_{2}$ with one base plane (Figure 5.4), thereby rendering that plane inaccessible for reactants. This is a conservative estimate, as the curvature of the $\mathrm{SiO}_{2}$ might in practice make these base planes partially accessible, which would increase the relative occurrence of hcp (0001). Furthermore, the dimensions of the as-synthesized NC were used in surface area calculations, i.e. disks with $d=15.5 \mathrm{~nm}$ and $\mathrm{h}=4.2 \mathrm{~nm}$ and spheres with $\mathrm{d}=10.7 \mathrm{~nm}$. 




Figure 5.8. Calculated accessible Co specific surface area (open bars) of $\mathrm{Co}-\mathrm{D} / \mathrm{SiO}_{2}$ and $\mathrm{Co}-\mathrm{S} / \mathrm{SiO}_{2}$ under FT conditions. The hcp (0001) surface area is indicated and broken down for the disk-shaped (red) and spherical (aqua) Co-NC.

Besides the surface of the disks, also the surface of the spheres has to be considered. According to Wulff reconstructions of spherical hep cobalt particles by Liu et al. ${ }^{25}, 18 \%$ of the surface of a spherical hep cobalt particle is terminated by (0001). Therefore, the maximal hcp (0001) occurrence in $\mathrm{Co}-\mathrm{S} / \mathrm{SiO}_{2}$ was $18 \%$. Compared to an estimated hcp(0001) occurrence of $39 \%$ in $\mathrm{Co}-\mathrm{D} / \mathrm{SiO}_{2}$, this means that $21 \%$ of the metallic cobalt surface area of $\mathrm{Co}-\mathrm{S} / \mathrm{SiO}_{2}$ is in a higher index and probably more active form than for the disk-containing $\mathrm{Co}-\mathrm{D} / \mathrm{SiO}_{2}$. This holds when all $\mathrm{NC}$ consist of hcp Co, while a difference in hcp (0001) exposure of $33 \%$ would be obtained if only disks were hcp Co. Considering the uncertainty and error margin in this analysis, we conclude that the difference in hep (0001) exposure could be of the right magnitude to account for much of the observed activity difference.

This is, to the best of our knowledge, the first time that a supported model catalyst has been synthesized to investigate the effect of $\mathrm{Co}-\mathrm{NC}$ shape in FT without complications of a secondary metal or interference of ligands and it illustrates the potential of colloidal techniques for model catalyst synthesis. Furthermore, based on these results, we tentatively propose that the extended low-index surface facets of the disks are the main contributor to the lower TOF.

\subsection{Conclusions}

We investigated the synthesis of disk-shaped cobalt nanocrystals (Co-NC), their attachment to a support and the subsequent effect of their anisotropy on the FischerTropsch synthesis. Disk-shaped hcp Co-NC were synthesized mixed with spherical \& CoNC. After oxidation to $\mathrm{CoO}$, these NC were attached to low surface area silica. Electron microscopy analysis revealed that numerically $66 \%$ of the attached $\mathrm{CoO}-\mathrm{NC}$ were disks and $34 \%$ were spheres. After in situ reduction of the $\mathrm{CoO}-\mathrm{NC}$ to $\mathrm{Co}$, the Fischer-Tropsch activity of disk-containing catalyst was only half that of a catalyst containing only spherical NC. Interestingly, the catalysts were stable and the size and shape of the NC were 
retained, even for the disk-shaped Co-NC. XPS showed that most of the amine ligands were removed during activation/FT and in situ XRD revealed that the crystal structure of both catalysts was similar, with $\mathrm{NC}$ with $3-5 \mathrm{~nm}$ polycrystalline domains and approximately $65 \%$ hcp Co and 21 to $32 \%$ fcc Co. Based on these experiments, we tentatively propose that changing the Co-NC shape from spheres to disks reduced FT activity because of hcp (0001) facets prevailing. This work clearly demonstrates the potential of colloidal methods to synthesize new, well-defined model catalysts to investigate structure-performance relationships in catalysis even under high-pressure conditions.

\section{Acknowledgements}

T.W.v.D., H.S., B.M.W. and K.P.d.J. acknowledge Shell Global Solutions and the Netherlands Association for Scientific Research (NWO) for funding through the CHIPP framework. H.Y., J.Z. and K.P.d.J. acknowledge the European Research Council, EU FP7 ERC Advanced Grant no. 338846. H.Y. acknowledges the Program for Advancing Strategic International Networks to Accelerate the Circulation of Talented Researchers by JSPS. We thank Nico Sommerdijk, Hans Meeldijk and Paul Bomans for their involvement with the (cryo-)TEM measurements, and Peter Munnik, Peter van den Brink, Sander van Bavel and Marco de Ridder from Shell Technology Center Amsterdam for XPS measurements and useful discussions.

\section{References}

1. Zečević, J., Vanbutsele, G., de Jong, K. P. \& Martens, J. A. Nanoscale intimacy in bifunctional catalysts for selective conversion of hydrocarbons. Nature 528, 245-248 (2015).

2. Cargnello, M. et al. Control of Metal Nanocrystal Size Reveals Metal-Support Interface Role for Ceria Catalysts. Science 341, 771-773 (2013).

3. Prieto, G., Zečević, J., Friedrich, H., De Jong, K. P. \& De Jongh, P. E. Towards stable catalysts by controlling collective properties of supported metal nanoparticles. Nat. Mater. 12, 34-39 (2013).

4. Munnik, P., de Jongh, P. E. \& de Jong, K. P. Recent Developments in the Synthesis of Supported Catalysts. Chem. Rev. 115, 6687-6718 (2015).

5. Jia, C.-J. \& Schüth, F. Colloidal metal nanoparticles as a component of designed catalyst. Phys. Chem. Chem. Phys. 13, 2457-2487 (2011).

6. Zaera, F. Nanostructured materials for applications in heterogeneous catalysis. Chem. Soc. Rev. 42, 2746-2762 (2013).

7. van Deelen, T. W., Su, H., Sommerdijk, N. A. J. M. \& de Jong, K. P. Assembly and activation of supported cobalt nanocrystal catalysts for the Fischer-Tropsch synthesis. Chem. Commun. 54, 2530-2533 (2018).

8. Yin, Y. \& Alivisatos, A. P. Colloidal nanocrystal synthesis and the organic-inorganic interface. Nature 437, 664-670 (2005).

9. Burda, C., Chen, X., Narayanan, R. \& El-Sayed, M. A. Chemistry and Properties of Nanocrystals of Different Shapes. Chem. Rev. 105, 1025-1102 (2005). 
10. Puntes, V. F., Zanchet, D., Erdonmez, C. K. \& Alivisatos, A. P. Synthesis of hep-Co nanodisks. J. Am. Chem. Soc. 124, 12874-12880 (2002).

11. Comesaña-Hermo, M. et al. Stable single domain Co nanodisks: synthesis, structure and magnetism. J. Mater. Chem. 22, 8043-8047 (2012).

12. van Santen, R. A. Complementary Structure Sensitive and Insensitive Catalytic Relationships. Acc. Chem. Res. 42, 57-66 (2009).

13. An, K. \& Somorjai, G. A. Size and Shape Control of Metal Nanoparticles for Reaction Selectivity in Catalysis. ChemCatChem 4, 1512-1524 (2012).

14. Roldan Cuenya, B. \& Behafarid, F. Nanocatalysis: Size- and shape-dependent chemisorption and catalytic reactivity. Surf. Sci. Rep. 70, 135-187 (2015).

15. Zaera, F. Shape-Controlled Nanostructures in Heterogeneous Catalysis. ChemSusChem 6, 1797-1820 (2013).

16. Zhou, Y., Li, Y. \& Shen, W. Shape Engineering of Oxide Nanoparticles for Heterogeneous Catalysis. Chem. - Asian J. 11, 1470-1488 (2016).

17. Torres Galvis, H. M. et al. Supported Iron Nanoparticles as Catalysts for Sustainable Production of Lower Olefins. Science 335, 835-838 (2012).

18. Chen, W. et al. Recent advances in the investigation of nanoeffects of Fischer-Tropsch catalysts. Catal. Today 311, 8-22 (2017).

19. Bezemer, G. L. et al. Cobalt Particle Size Effects in the Fischer-Tropsch Reaction Studied with Carbon Nanofiber Supported Catalysts. J. Am. Chem. Soc. 128, 3956-3964 (2006).

20. den Breejen, J. P. et al. On the Origin of the Cobalt Particle Size Effects in Fischer-Tropsch Catalysis. J. Am. Chem. Soc. 131, 7197-7203 (2009).

21. Prieto, G., Martínez, A., Concepción, P. \& Moreno-Tost, R. Cobalt particle size effects in Fischer-Tropsch synthesis: structural and in situ spectroscopic characterisation on reverse micelle-synthesised Co/ITQ-2 model catalysts. J. Catal. 266, 129-144 (2009).

22. Ducreux, O., Rebours, B., Lynch, J., Roy-Auberger, M. \& Bazin, D. Microstructure of Supported Cobalt Fischer-Tropsch Catalysts. Oil Gas Sci. Technol. - Rev. d'IFP Energies Nouv. 64, 49-62 (2009).

23. Sadeqzadeh, M. et al. Identification of the active species in the working alumina-supported cobalt catalyst under various conditions of Fischer-Tropsch synthesis. Catal. Today 164, 62-67 (2011).

24. Gnanamani, M. K., Jacobs, G., Shafer, W. D. \& Davis, B. H. Fischer-Tropsch synthesis: Activity of metallic phases of cobalt supported on silica. Catal. Today 215, 13-17 (2013).

25. Liu, J.-X., Su, H.-Y., Sun, D.-P., Zhang, B.-Y. \& Li, W.-X. Crystallographic Dependence of CO Activation on Cobalt Catalysts: HCP versus FCC. J. Am. Chem. Soc. 135, 16284-16287 (2013).

26. Lyu, S. et al. Role of Active Phase in Fischer-Tropsch Synthesis: Experimental Evidence of CO Activation over Single-Phase Cobalt Catalysts. ACS Catal. 8, 7787-7798 (2018).

27. Rebmann, E., Fongarland, P., Lecocq, V., Diehl, F. \& Schuurman, Y. Measurement of the Influence of the Microstructure of Alumina-Supported Cobalt Catalysts on their Activity and Selectivity in Fischer-Tropsch Synthesis by using Steady-State and Transient Kinetics. ChemCatChem 9, 2344-2351 (2017).

28. van Helden, P., Ciobîcă, I. M. \& Coetzer, R. L. J. The size-dependent site composition of FCC cobalt nanocrystals. Catal. Today 261, 48-59 (2016). 
29. Agrawal, R., Phatak, P. \& Spanu, L. Effect of phase and size on surface sites in cobalt nanoparticles. Catal. Today 312, 174-180 (2018).

30. Geerlings, J. J. C., Zonnevylle, M. C. \& de Groot, C. P. M. Structure sensitivity of the FischerTropsch reaction on cobalt single crystals. Surf. Sci. 241, 315-324 (1991).

31. Geerlings, J. J. C., Zonnevylle, M. C. \& de Groot, C. P. M. Studies of the Fischer-Tropsch reaction on Co(0001). Surf. Sci. 241, 302-314 (1991).

32. Qin, C. et al. Crystal-Plane-Dependent Fischer-Tropsch Performance of Cobalt Catalysts. ACS Catal. 8, 9447-9455 (2018).

33. Oosterbeek, H. Bridging the pressure and material gap in heterogeneous catalysis: cobalt Fischer-Tropsch catalysts from surface science to industrial application. Phys. Chem. Chem. Phys. 9, 3570-3576 (2007).

34. Navarro, V., van Spronsen, M. A. \& Frenken, J. W. M. In situ observation of self-assembled hydrocarbon Fischer-Tropsch products on a cobalt catalyst. Nat. Chem. 8, 929-934 (2016).

35. Venvik, H. J., Borg, A. \& Berg, C. Formation of the CO-induced ( 3 x 1) surface structure on Co(11-20) studied by STM. Surf. Sci. 397, 322-332 (1998).

36. Strømsheim, M. D. et al. Effects of K adsorption on the CO-induced restructuring of Co(11-20). Catal. Today 299, 37-46 (2018).

37. Schulz, H., Nie, Z. \& Ousmanov, F. Construction of the Fischer-Tropsch regime with cobalt catalysts. Catal. Today 71, 351-360 (2002).

38. Wilson, J. \& de Groot, C. P. M. Atomic-Scale Restructuring in High-Pressure Catalysis. J. Phys. Chem. 99, 7860-7866 (1995).

39. Banerjee, A. et al. Shape and Size of Cobalt Nanoislands Formed Spontaneously on Cobalt Terraces during Fischer-Tropsch Synthesis. J. Phys. Chem. Lett. 7, 1996-2001 (2016).

40. Høydalsvik, K. et al. Morphology Changes of Co Catalyst Nanoparticles at the Onset of Fischer-Tropsch Synthesis. J. Phys. Chem. C 118, 2399-2407 (2014).

41. Weststrate, C. J. et al. Atomic and Polymeric Carbon on Co(0001): Surface Reconstruction, Graphene Formation, and Catalyst Poisoning. J. Phys. Chem. C116, 11575-11583 (2012).

42. Zhong, L. et al. Cobalt carbide nanoprisms for direct production of lower olefins from syngas. Nature 538, 84-87 (2016).

43. Scariot, M. et al. Cobalt Nanocubes in Ionic Liquids: Synthesis and Properties. Angew. Chem. Int. Ed. 47, 9075-9078 (2008).

44. Ba, R. et al. Synthesis of Co-based bimetallic nanocrystals with one-dimensional structure for selective control on syngas conversion. Nanoscale 7, 12365-12371 (2015).

45. Wen, C., Dunbar, D., Zhang, X., Lauterbach, J. \& Hattrick-Simpers, J. Self-healing catalysts: $\mathrm{Co}_{3} \mathrm{O}_{4}$ nanorods for Fischer-Tropsch synthesis. Chem. Commun. 50, 4575-4578 (2014).

46. Harmel, J. et al. A Seed-Mediated Approach for the Preparation of Modified Heterogeneous Catalysts. ChemCatChem 10, 1614-1619 (2018).

47. Harmel, J. et al. hcp-Co nanowires grown on metallic foams as catalysts for the FischerTropsch synthesis. Angew. Chem. Int. Ed. 57, 10579-10583 (2018).

48. Puntes, V. F., Krishnan, K. M. \& Alivisatos, A. P. Colloidal Nanocrystal Shape and Size Control: The Case of Cobalt. Science 291, 2115-2117 (2001). 
49. van Deelen, T. W., Nijhuis, J. J., Krans, N. A., Zečević, J. \& de Jong, K. P. Preparation of Cobalt Nanocrystals Supported on Metal Oxides To Study Particle Growth in Fischer-Tropsch Catalysts. ACS Catal. 8, 10581-10589 (2018).

50. Reuel, R. C. \& Bartholomew, C. H. The Stoichiometries of $\mathrm{H}_{2}$ and $\mathrm{CO}$ Adsorptions on Cobalt: Effects of Support and Preparation. J. Catal. 85, 63-77 (1984).

51. Leijten, Z. J. W. A., Keizer, A. D. A., de With, G. \& Friedrich, H. Quantitative Analysis of Electron Beam Damage in Organic Thin Films. J. Phys. Chem. C121, 10552-10561 (2017).

52. Kremer, J. R., Mastronarde, D. N. \& McIntosh, J. R. Computer Visualization of ThreeDimensional Image Data Using IMOD. J. Struct. Biol.116, 71-76 (1996).

53. Schindelin, J. et al. Fiji: An open-source platform for biological-image analysis. Nat. Methods 9, 676-682 (2012).

54. Cats, K. H. \& Weckhuysen, B. M. Combined Operando X-ray Diffraction/Raman Spectroscopy of Catalytic Solids in the Laboratory: The $\mathrm{Co} / \mathrm{TiO}_{2}$ Fischer-Tropsch Synthesis Catalyst Showcase. ChemCatChem 8, 1531-1542 (2016).

55. Xie, J. et al. Promoted cobalt metal catalysts suitable for the production of lower olefins from natural gas. Nat. Commun. 10, 167 (2019).

56. Puntes, V. F., Krishnan, K. \& Alivisatos, A. P. Synthesis of colloidal cobalt nanoparticles with controlled size and shapes. Top. Catal. 19, 145-148 (2002).

57. Munnik, P., de Jongh, P. E. \& de Jong, K. P. Control and Impact of the Nanoscale Distribution of Supported Cobalt Particles used in Fischer-Tropsch Catalysis. J. Am. Chem. Soc. 136, 73337340 (2014).

58. Eschemann, T. O. et al. Effect of support surface treatment on the synthesis, structure, and performance of Co/CNT Fischer-Tropsch catalysts. J. Catal. 328, 130-138 (2015).

59. Sławiŕski, W. A., Zacharaki, E., Fjellvåg, H. \& Sjåstad, A. O. Structural Arrangement in ClosePacked Cobalt Polytypes. Cryst. Growth Des. 18, 2316-2325 (2018).

60. Andreev, A. S., d'Espinose de Lacaillerie, J.-B., Lapina, O. B. \& Gerashenko, A. Thermal stability and hcp-fcc allotropic transformation in supported Co metal catalysts probed near operando by ferromagnetic NMR. Phys. Chem. Chem. Phys. 17, 14598-14604 (2015).

61. Srinivasan, R., De Angelis, R. J., Reucroft, P. J., Dhere, A. G. \& Bentley, J. Structural Characterization of Cobalt Catalysts on a Silica Support. J. Catal. 116, 144-163 (1989).

62. Garces, L. J., Hincapie, B., Zerger, R. \& Suib, S. L. The Effect of Temperature and Support on the Reduction of Cobalt Oxide: An in Situ X-ray Diffraction Study. J. Phys. Chem. C119, 54845490 (2015). 
Disk-shaped cobalt nanocrystals as Fischer-Tropsch synthesis catalysts under industrially relevant conditions 



\title{
Chapter 6
}

\section{Control of metal-support interactions in heterogeneous catalysts to enhance activity and selectivity}

\begin{abstract}
Metal nanoparticles stabilized on a support material catalyze many major industrial reactions. Metal-support interaction (MSI) in these nanomaterials can have a substantial influence on the catalysis, making MSI modulation one of the few tools to enhance catalytic performance. This topic has received much attention in recent years, however, a systematic rationalization of the field is lacking due to the great diversity in catalysts, reactions and MSI modification strategies. In this review chapter, we cover and categorize the recent progress in MSI tuning strategies to enhance catalytic performance for various reactions. Furthermore, we quantify the productivity enhancements resulting from MSI control that have been achieved in $C_{1}$ chemistry in recent years. Our analysis shows that up to 15-fold productivity enhancement has been achieved and that MSI is most impactful for metal nanoparticles smaller than $4 \mathrm{~nm}$. These findings demonstrate the importance of MSI to improve performance in catalysis.
\end{abstract}

Based on: van Deelen, T. W.", Hernández Mejía, C.* \& de Jong, K. P. Nat. Catal. 2, 955-970 (2019).

\# Both authors contributed equally to this manuscript. 


\subsection{Introduction}

Metal nanoparticles are the key functional component in various nanotechnological applications, owing to the unique properties that arise from their size, shape and composition. ${ }^{1,2}$ Consequently, several strategies have been developed to design and control these properties. A key strategy is to immobilize nanoparticles on a support to enhance their stability and control their spatial distribution. ${ }^{3,4}$ However, supports are typically not inert and the interaction with nanoparticles gives rise to new interface phenomena. Such metal-support interaction (MSI) may have a profound impact on the resulting performance of the metal nanoparticles. ${ }^{5,6}$ Hence, strategies for controlling these interactions to optimize performance are highly desirable.

In heterogeneous catalysis, MSI affects the catalytic performance and its regulation thus provides options for catalyst design. Recently, the topic has gained much attention as judged by the increasing number of reports on the subject and substantial progress has been made in methodology to tune metal-support interactions with the aim to improve the performance of supported metal catalysts. Because of the great diversity in MSI tuning approaches, catalytic systems and reactions, a systematic and critical overview would be helpful to determine the most effective tuning strategies for real working catalysts.

This chapter starts with a brief introduction covering the relevant concepts of MSI phenomena and the following sections discuss the latest reports on MSI tuning strategies for catalysis, systematically arranged after the different stages of catalyst modification. Several interesting reviews have already been published in the field of MSI. ${ }^{7-10}$ In comparison, we have reviewed the literature published since 2015 on tuning interactions between metals and supports to improve catalytic performance, covering mainly 3D catalysts containing metal nanoparticles or clusters under industrially relevant conditions. For a general discussion, we provide a quantitative analysis of the scale of performance enhancement that has been reported in the past years. To quantify the effects of MSI modulation on catalytic performance, we focus on intrinsic activity and selectivity as performance descriptors and less on stability. Although highly relevant from an application point of view, activity enhancement due to changes in metal dispersion (increased metal specific surface area) are not covered here. Relative scaling factors are obtained by comparing the productivities of tuned and reference catalysts in $C_{1}$ chemistry, which allow us to point out the most successful tuning strategies and opportunities in this field.

\subsection{Metal-support interactions}

Impact on catalysis originates from interactions between metal nanoparticles and their supporting matrix (Figure 6.1Error! Reference source not found.a). Typical metalsupport phenomena relate to charge transfer, the interfacial perimeter, nanoparticle morphology, chemical composition and strong metal-support interaction (SMSI). These phenomena are often entangled and some will have a stronger influence than others, depending on the catalyst and the reaction. 
a

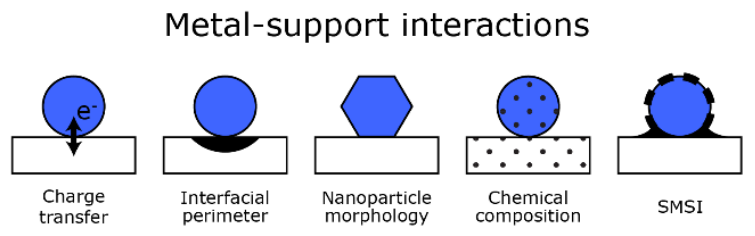

b
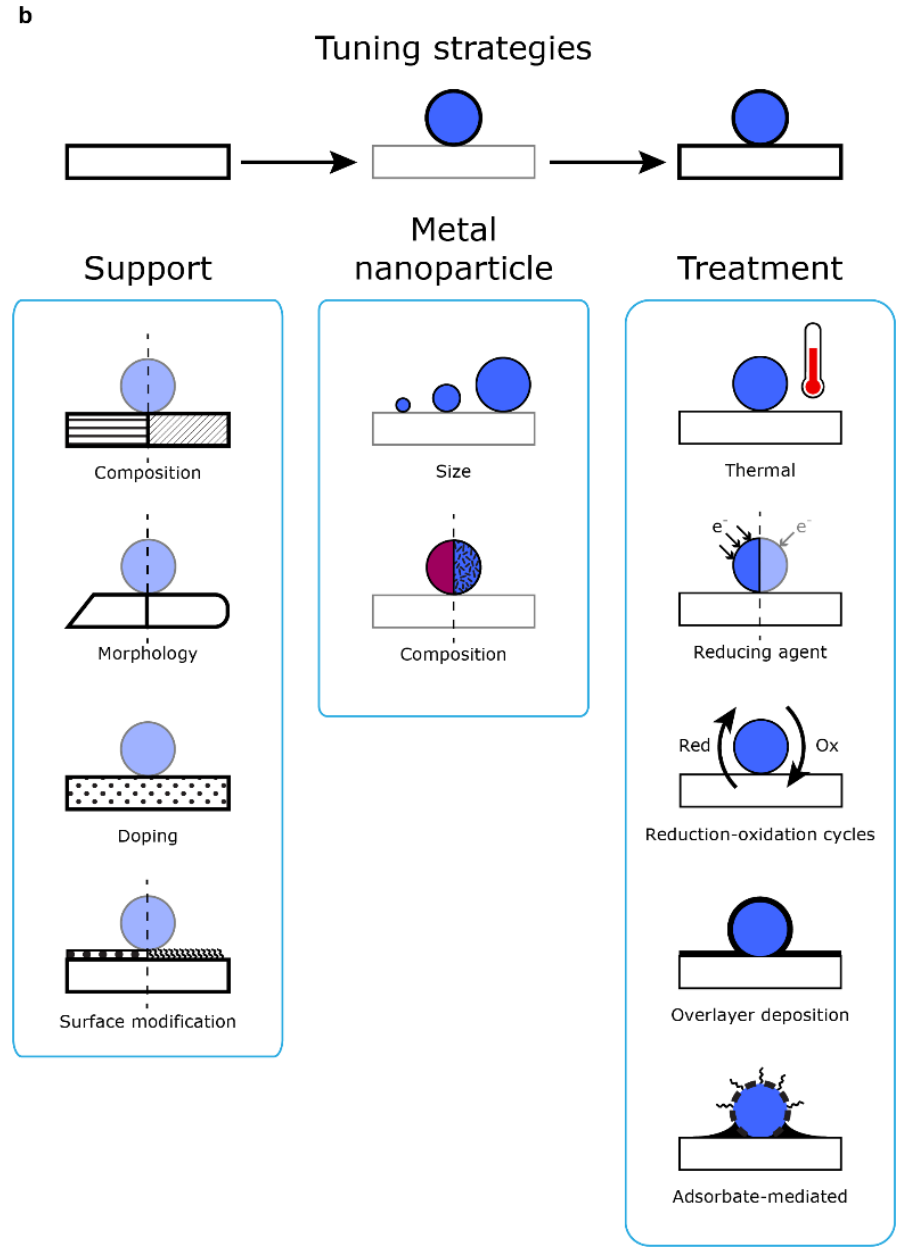

Figure 6.1. Schematic representation of main metal-support interactions and their tuning strategies. The five main phenomena of metal-support interactions are depicted in a. The approaches for tuning such interactions are illustrated in $\mathbf{b}$ and divided after the different stages of catalyst modification, i.e., via solely the support or the nanoparticle or via treatments on the metalsupport composite material.

Charge transfer. The interface between a metal NP and the support can give rise to a rearrangement of electrons within both materials. ${ }^{5}$ Redistribution of electrons with noticeable effects is limited to a couple of atomic layers at the interface and might be accompanied in some cases by a change in oxidation state of the metal atoms from the NP or of the support's metal ions. ${ }^{11,12}$ The magnitude and direction of the charge transfer is driven by differences in the Fermi level of the metal NP and the support, ultimately seeking equilibration of the electron chemical potentials. The metallic character of the NPs enables electron mobility, however their size is relevant in the nanometer regime, since the smaller 
the NP the more localized are its electronic states. Several characteristics of the support are important for charge transfer, such as its conductivity, reducibility, exposed crystal planes, morphology and occurrence of defects..$^{13,14}$

Interfacial perimeter. Interface sites at the perimeter of the NP are a unique environment given that they are in direct contact with the NP, the support and the reactants, facilitating synchronized reactions. ${ }^{15,16}$ Furthermore, it has been shown that upon charge transfer, atoms at the perimeter favor the accumulation of excess charges. ${ }^{17}$ All this can significantly enhance adsorption and reactions of molecules at the perimeter. ${ }^{18,19}$ Additionally, close proximity of the nanoparticle to different functionalities or defects at the surface of the support (e.g., oxygen vacancies, hydroxyl groups, Lewis acids or bases) might also aid local sequential reactions or stabilize transition states of reactants or products. ${ }^{20,21}$

Spillover via the interfacial perimeter can occur as well. This initiates with the activation of a reactant on one surface, typically on the metal NP, which is then transferred to the other surface, e.g., the support. Note that this second surface does not activate the reactants itself under the same conditions. The most studied spillover is for $\mathrm{H}$ and to a lesser extent $\mathrm{O}$ or other molecules (e.g., $\mathrm{CO},-\mathrm{OCH}_{3}$, etc.). Spillover from the support to the metal nanoparticle can also occur, referred sometimes as reverse spillover. ${ }^{22-24}$ The nature of the support is crucial for spillover; whereas hydrogen spillover on reducible supports occurs fast and at relative large distances, it has been shown to proceed ten orders of magnitude slower on non-reducible supports and is restricted to short distances. ${ }^{25}$

Nanoparticle morphology. The shape and crystal structure of nanoparticles have a strong influence on their catalytic performance, considering that different shapes expose certain facets and these facets can have favorable or unfavorable configurations of atoms. ${ }^{26,27}$ The adhesion energy at the metal-support interface affects the shape of the nanoparticles. ${ }^{26,28}$ In general, supports with stronger adhesion result in particles with more faceted and sometimes raft-like shapes. ${ }^{29}$ A trend observed for metal oxide supports suggests that adhesion energies increase with increasing heat of formation of the most stable oxide of the metal ${ }^{30}$ and by decreasing the metal NP's size ${ }^{31}$. Besides NP shape, high adhesion energy will also reduce the mobility of the NPs and hence their tendency to grow. ${ }^{6,32}$

The equilibrium shape of a metal NP is often determined by minimization of its total surface free energy. Contact with the support can lower the surface energy of certain NP's planes, favoring some shapes over others. The surface energy of a metal can also change under reaction conditions upon adsorption of a gas, where the change in its surface energy tends to affect the contact surface area with the NP and hence modifies the NP's shape. ${ }^{33,34}$ Moreover, a misfit between the lattices of the support and the nanoparticle can generate strain and defects, modifying the morphology of the NP. ${ }^{35}$ Additionally, the effect of lattice mismatching is more pronounced for smaller metal nanoparticles, as observed for gold nanoparticles smaller than $3 \mathrm{~nm}$ supported on titania. ${ }^{36}$

Chemical composition. A solid-state reaction can take place between metal NPs and the support resulting in the formation of new phases. Exchange of species is possible in both 
directions, usually coupled with a redox process, with oxidation of the metal atoms from the nanoparticle or reduction of metal ions from the support. This phenomenon has ambivalent consequences, since the resulting species can both lower or enhance catalytic performance. On the one hand, formation of inactive phases such as mixed metal oxides (e.g., metal aluminates) at the expense of active metal sites has been recognized for long time as a prominent deactivation process. ${ }^{37,38}$ On the other hand, formation of highly active intermetallic nanoparticles is also possible by reduction of metal or metalloid ions from the support to incorporate into the metal NP. This phenomenon has recently attracted significant attention and is sometimes referred as reactive metal-support interaction (RMSI) ${ }^{39}$ The pre-deposited metal nanoparticles aid in the activation of the support. Upon high temperatures and reductive conditions, the former cations from the support can migrate to the nanoparticle. This can be facilitated by doping the support or employing 2D supports. The strategy has been effectively applied to incorporate difficult-to-reduce metals or metalloids (e.g., Si, Al, V, Ti, Nb, etc.) into late transition metal NPs, resulting in novel catalytic systems. ${ }^{40}$

The local composition of alloyed metal nanoparticles can be affected by interacting with the support, where re-arrangement of the components in an alloyed nanoparticle is driven by preferential interaction of one of its elements with the support at the interface. ${ }^{41}$ This can lead to compositional rearrangements different from the initially uniform composition, such as core-shell or segregated sub-cluster structures, affecting the synergy of the metals and hence their catalytic performance. ${ }^{42,43}$

Strong metal-support interaction. The term strong metal-support interaction (SMSI) refers to metal NP coverage by suboxides, which are generated from the support under reducing conditions. ${ }^{44,45}$ The phenomenon is driven by minimization of the high surface energy of the metal NP by mobile support suboxide species. High surface energy metals that are capable of activating hydrogen are prone to encapsulation and reducible supports are necessary to generate the suboxide species. The suboxides covering the nanoparticle consist of a few atomic layers with probably amorphous character and possess dynamic structures under different gas atmospheres as shown in the case of titanium and iron oxide. ${ }^{46-48}$ Extensive coverage of the NP is detrimental for the catalytic performance due to blockage of active sites. However, these suboxides can change the (local) electronic structure of the metal surface and act as Lewis acids, thereby promoting the activation of reactants and improve the catalytic performance. ${ }^{49}$

\subsubsection{Modification of metal-support interactions}

The MSI phenomena can have a major impact on the catalytic performance. Therefore, modification of MSI can be employed as a tool for the design of catalytic systems with enhanced performance. Several synthetic strategies for the control of the MSI have been reported, which can be classified according to changes to the catalyst's components before and after assembly of the composite material (Figure 6.1b). Through this distinction, we aim to emphasize the similarities within one strategy, when applied to different families of catalysts, supports and reactions. In section 3 we start by discussing reports targeting modification mainly of the support, divided into strategies involving support composition, 
morphology, doping and surface modifications. In section 4, strategies that focus on nanoparticle modifications are discussed, particularly changes in NP's size and composition. In section 5, we cover the approaches to tune MSI on as-prepared catalysts by use of treatments. The identified treatments include thermal, reducing agent, reduction-oxidation cycles, overlayer deposition and adsorbate-mediated treatments.

\subsection{Support}

The first approach to tune the metal-support interactions is through a judicious choice of support characteristics. The support provides numerous opportunities for changing its structure and surface composition, making it one of the most employed handles to tune MSI. In this section, we will discuss modifications of supports prior to deposition of NPs. Support tuning strategies after NP loading are classified as a post-synthesis treatment and will be discussed in the Treatment section.

\subsubsection{Support composition}

The first point of consideration is the chemical composition of the support, as that partially defines the starting point for MSI. The discussion of support composition involves comparisons between different types of supports as well as between different chemical compositions of related support compounds. Examples of support properties impacting MSI include the surface composition of the support (such as extent of hydroxylation), the electronic structure of the support (especially important for electro- or photocatalysis), ion mobility (e.g., formation of oxygen vacancies or cation diffusion), reactivity of the support, and the possibility to segregate cations to form intermetallic compounds.

Several recent publications investigated the role of the support in the catalytic oxidation of $\mathrm{CO}$ by $\mathrm{Au}$ NPs. Saavedra et al. ${ }^{50}$ studied in detail the origin of lower $\mathrm{CO}$ oxidation activity at room temperature of $\mathrm{Au} \mathrm{NP}$ supported on $\mathrm{Al}_{2} \mathrm{O}_{3}$ compared to $\mathrm{TiO}_{2}$. They concluded that activity was to a large extent determined by competitive adsorption of water (promoting) and carbonates (poisoning) on the support and active metal sites. The role of the support was mainly to supply adsorbed water to the Au NPs. This was more efficient on $\mathrm{TiO}_{2}$, given that carbonates bind stronger to $\mathrm{Al}_{2} \mathrm{O}_{3}$ than to $\mathrm{TiO}_{2}$ and they propose that oxygen vacancies do not participate significantly in the catalysis. Contrary, Wang et al. ${ }^{51,52}$ reported that oxygen vacancies are generated in $\mathrm{TiO}_{2}$ and that these vacancies are mainly responsible for the modified catalytic activity. The vacancies caused overall electron donation to the Au NPs, making these NP negatively charged. The charge donation resulted in strongly modified CO adsorption strength and lower intrinsic activity at $80{ }^{\circ} \mathrm{C}$. Interestingly, the TOF could be enhanced by one order of magnitude by re-oxidation of the catalyst, which reduced the amount of oxygen vacancies. One possibility for these two different explanations of the same catalytic system could be the different pre-treatment or reaction conditions, such as reaction temperature (RT vs. $80^{\circ} \mathrm{C}$ ), feed composition (1 vs $20 \% \mathrm{O}_{2}$ ), or dilution with inert $\mathrm{SiC}$. For example, it has been reported that the mechanism of $\mathrm{CO}$ oxidation changes with reaction temperature..$^{2}$

The activity for benzyl alcohol oxidation followed the opposite trend as for CO oxidation with the intrinsic activity decreasing in the order 
$\mathrm{Au} / \mathrm{Al}_{2} \mathrm{O}_{3}>\mathrm{Au} / \mathrm{SiO}_{2}>\mathrm{Au} / \mathrm{TiO}_{2}>\mathrm{Au} / \mathrm{ZnO}$ (Figure 6.2a). ${ }^{53}$ Charge transfer occurred to a different extent from various supports to Au NPs of 2-4 nm size and correlated inversely with the electron donation capacity of the support. Hydride transfer from the alcohol to $\mathrm{Au}$ was argued to be the rate determining in this case and would account for lower activity of more negatively charged $\mathrm{Au} \mathrm{NP}$.

In photocatalysis, a semiconductor support's bandgap of appropriate size and position facilitates light absorption and transfer of the generated charge to or from metal NPs performing catalysis. The activity of $\mathrm{Rh} / \mathrm{TiO} 2$ in steam reforming of methane was already 13 times higher than that of $\mathrm{Rh} / \mathrm{SiO} 2$ and $\mathrm{Rh} / \mathrm{ZrO} 2$ in dark conditions and the activity of $\mathrm{Rh} / \mathrm{TiO} 2$ was enhanced further nearly 3 times when exposed to light (Figure 6.2b). ${ }^{54}$ However, the opposite has been observed as well; $\mathrm{Pt} / \mathrm{Ta}_{2} \mathrm{O}_{5}$ was more active than $\mathrm{Pt} / \mathrm{TiO}_{2}$ for 2-propanol oxidation to acetone, because hot electrons generated on the Pt NPs were not transferred to the support and hence drove the catalysis on the Pt NPs. ${ }^{55}$ XPS analysis indicated that the Pt phase was more negatively charged on $\mathrm{Ta}_{2} \mathrm{O}_{5}$ than on other supports. Similar considerations also apply to electrocatalysis. The activity of Pt NPs for electrocatalytic reduction of oxygen was significantly enhanced when a mixture of boron carbide and graphite was used as support instead of pure graphite and it was argued that this was solely an electronic effect. ${ }^{56}$

Perovskites are a class of metal oxides with the general formula $\mathrm{ABO}_{3}$ in which a large variety of metal ions can be incorporated in the A- and B-sites of their crystal structures. Some of these ions can be extracted from the perovskite lattice upon reduction to form nanoparticles. This approach has received great attention for electrochemical systems ${ }^{57}$ mainly due to the beneficial conductive properties of perovskites for electrodes. However, this approach has been employed to a lesser extent for catalysis due to the resulting low metal loading and limitation to easy-to-reduce metal ions, such as noble metals. Recently, non-stoichiometric perovskites with A-site deficiency extended the range of metal ions capable of forming nanoparticles to more difficult to reduce metal ions (e.g., $\mathrm{Ti}^{4+}, \mathrm{Fe}^{2+/ 3+}$, $\left.\mathrm{Mn}^{2+/ 3+}, \mathrm{Ni}^{2+}\right) .{ }^{58,59}$ The resulting nanoparticles resisted the harsh conditions of methane reforming due to partial embedding in the support, avoiding the formation of carbon fibers at the periphery of the nanoparticles. Formation of alloyed nanoparticles is also possible by use of this strategy. Alloyed $\mathrm{Pt}_{3} \mathrm{Ni}$ nanoparticles obtained from $\mathrm{La}_{0.9} \mathrm{Mn}_{0.9} \mathrm{Pt}_{0.075} \mathrm{Ni}_{0.025} \mathrm{O}_{\mathrm{x}}$ perovskite have shown high activity for the electrocatalytic oxygen reduction reaction. ${ }^{60}$ The strong connection with the conductive perovskite, uniform NP distribution and small NP size for the obtained particles were beneficial to the measured activity.

The disadvantage, however, of using perovskites to form nanoparticles in catalysis is the low specific surface area of these compounds, due to the high temperatures needed to synthesize the perovskites. Progress has been achieved in fabricating high surface area perovskites by templating methods, however the challenge remains with the high temperatures needed to form the metal nanoparticles, which usually leads to collapse of the pore structure of the perovskites. ${ }^{61}$

\subsubsection{Support morphology}

Various allotropes or morphologies can exist for one type of support. We will discuss these two properties together, because they are often interrelated and exert a combined effect. 
For example, the crystal structure and morphology of the support jointly determine the facets that are exposed at the surface of the support and thereby regulate the local environment for the metal nanoparticles. In this section, we will highlight some recent examples of how the crystal structure of metal oxides can direct NP shape and SMSI, and how shaped support particles can expose special surface sites or confine NPs.

The crystal structure of metal oxides can affect the NPs deposited onto it by directing the shape of the metal NPs. For example, when $\mathrm{Pd} / \mathrm{Al}_{2} \mathrm{O}_{3}(\alpha, \gamma$, and $\theta)$ catalysts were investigated for methane oxidation, the crystal structure of the support affected the shape of Pd NPs, with more distorted NP on $\mathrm{Y}-\mathrm{Al}_{2} \mathrm{O}_{3}$ and more regularly shaped and facetted NP on $\alpha-$ and $\theta-\mathrm{Al}_{2} \mathrm{O}_{3} .{ }^{62}$ The shape of the NP together with NP size determined the number of step sites, which could be correlated with intrinsic activity. The presence of pentacoordinate $\mathrm{Al}^{3+}$-sites on the surface of $\gamma^{-} \mathrm{Al}_{2} \mathrm{O}_{3}$, as observed by NMR, and their absence in $\alpha$ - and $\theta-\mathrm{Al}_{2} \mathrm{O}_{3}$ were proposed to distort the NP shape and increase the TOF of $7.5 \mathrm{~nm}$ NP from 0.1 to $0.6 \mathrm{~s}^{-1}$. A similar effect was observed for Pt NPs on $\mathrm{Y}-\mathrm{Al}_{2} \mathrm{O}_{3}$ and $\mathrm{CeO}_{2} \cdot{ }^{35}$ Especially on $\mathrm{CeO}_{2}$, the authors were able to show that epitaxial NP growth, dictated by the support crystal structure, directed the shape of the NPs and caused strain. From theoretical investigations, the predicted intrinsic activity for $\mathrm{CO}$ oxidation varied between $2 \cdot 10^{-3}$ and $5 \cdot 10^{-3} \mathrm{~s}^{-1}$ with the occurrence of specific Pt surface sites, related to the shape and strain of these NPs.

In addition, one crystal structure can be more susceptible to partial reduction and SMSI. In the case of $\mathrm{TiO}_{2}$-supported $\mathrm{Ru}$-promoted cobalt catalysts for the Fischer-Tropsch synthesis ${ }^{63}$ and rhodium catalysts for steam reforming of propane ${ }^{64}$, suboxide coverage was more extensive on $\mathrm{TiO}_{2}$ in the anatase phase than in the rutile phase. In both cases, the overall activity of the catalysts was higher on rutile, because of fewer blockages of active sites by the $\mathrm{TiO}_{\mathrm{x}}$ species. Furthermore, the specific surface area of the $\mathrm{TiO}_{2}$-anatase support was shown to also influence the extent of SMSI and activity on $\mathrm{Ru}-\mathrm{Co} / \mathrm{TiO}_{2}$ catalysts for the Fischer-Tropsch synthesis ${ }^{65}$ and $\mathrm{Ru} / \mathrm{TiO}_{2}$ catalysts for $\mathrm{CO}$ methanation ${ }^{66}$. The cobaltweight-based activity decreased for both catalysts with increasing specific surface area of the support, again because of a larger number of blocked sites. The TOF was, however, not affected by the support specific surface area. In this case, the anatase supports with higher surface area displayed a higher concentration of defects, as inferred from Raman spectroscopy, and this probably led to more facile formation of $\mathrm{TiO}_{\mathrm{x}}$ species during reduction.

The shape of the support, i.e., the exposed crystal facets, also influence the catalytic performance through MSI. The activity of $\mathrm{Pt} / \mathrm{TiO}_{2}$ for methanol oxidation ${ }^{67}, \mathrm{Co} / \mathrm{CeO}_{2}$ and $\mathrm{Ru} / \mathrm{CeO}_{2}$ for ammonia synthesis ${ }^{68,69}$ and $\mathrm{Au} / \mathrm{CeO}_{2}$ (Figure 6.2c) and $\mathrm{Au} / \mathrm{ZnO}$ for $\mathrm{CO}$ oxidation $^{70,71}$ showed large differences depending on the facets on which the metal NPs were situated. The facets were controlled by synthesizing nanostructured support particles of controlled shapes. The reason for the modified catalytic performance varied, however, from facilitated reactant/intermediate binding and charge transfer $\left(\mathrm{Pt} / \mathrm{TiO}_{2}\right)$, enhanced support reduction ( $\mathrm{Co}$ and $\mathrm{Ru}$ on $\mathrm{CeO}_{2}$ ) to the occurrence of more vacancies $\left(\mathrm{Au}\right.$ on $\mathrm{CeO}_{2}$ and $\mathrm{ZnO}$ ). For $\mathrm{Au} / \mathrm{ZnO}$, the vacancies allowed $\mathrm{Au}$ to replace $\mathrm{Zn}$ in the $\mathrm{ZnO}$ crystal lattice and promote a Mars-van Krevelen mechanism through the formation of $\mathrm{Au}-\mathrm{O}-\mathrm{Au}_{\mathrm{Zn}}$ bonds, 
which are broken easier than $\mathrm{Au}-\mathrm{O}-\mathrm{Zn}$ bonds. This led to a remarkable 150 times increase in specific activity that was amplified using a very low activity reference catalyst.

One- and two-dimensional supports can give rise to confinement or exposure of special sites on the support surface. For example, Zhu et al. ${ }^{72}$ demonstrated enhanced CO oxidation activity for Pt NPs supported on boron nitride nanosheets. The NPs associated to $\mathrm{B}$-vacancies displayed lower electron density while N-vacancies donated electron density to Pt-NPs. Since the N-vacancies were the main Pt-binding sites, the Pt NPs were electron rich compared to those supported on bulk boron nitride, which contained less vacancies. This enhanced $\mathrm{O}_{2}$ adsorption and increased $\mathrm{CO}$ oxidation activity from 30 to $70 \%$ conversion at $47^{\circ} \mathrm{C}$. Similarly, the selectivity towards $\mathrm{CH}_{4}$ of Ni NPs on planar siloxenes in $\mathrm{CO}_{2}$ hydrogenation was $90 \%$ when the NPs were confined in the interior of the siloxenes structures and $53 \%$ when the Ni NPs were located on the exterior of the support. ${ }^{73}$ In this case, confinement tuned the balance between the formate and the CO intermediate reaction pathway. Another example is confinement of Pt NPs in the interior of carbon nanotubes (CNT), which reduced NP size and prevented oxidation of Pt. ${ }^{74}$ This kept the metal in the active state and increased toluene oxidation activity from 4 to $27 \mu \mathrm{mol} \cdot \mathrm{g}_{\mathrm{Pt}}{ }^{-1} \cdot \mathrm{s}^{-1}$ at $140{ }^{\circ} \mathrm{C}$ compared to NPs located on the exterior surface of the CNT.

Two-dimensional early transition metal carbides present weaker metal-carbon bonds, due to their dimensionality, compared to bonds in traditional metal oxides or metal carbides. This results in increased chemical reactivity of the metal ions and facilitating RMSI. A two-dimensional niobium carbide $\mathrm{Nb}_{2} \mathrm{CT}_{\mathrm{x}}$ (where $\mathrm{T}$ accounts for various elements/groups) has been used as support for platinum nanoparticles and applied for the water-gas shift reaction. ${ }^{75}$ The two-dimensionality of this carbide material aided in the formation of a $\mathrm{Pt}-\mathrm{Nb}$ surface alloy by $\mathrm{H}_{2}$ reduction at $350{ }^{\circ} \mathrm{C}$. Furthermore, the removal of functional groups $(\mathrm{T})$ such as $-\mathrm{OH},-\mathrm{O}$ and $-\mathrm{F}$, exposed terminal niobium sites which were identified as interfaces for $\mathrm{H}_{2} \mathrm{O}$ activation. These exposed niobium atoms in contact with the $\mathrm{Pt}-\mathrm{Nb}$ surface alloy showed strong affinities for $\mathrm{H}_{2} \mathrm{O}$ or $\mathrm{OH}$, as indicated by the WGS kinetics. However, this catalyst showed a similar reaction rate of $\sim 1.5 \cdot 10^{-2} \mathrm{~mol}_{\mathrm{H} 2} \cdot \mathrm{mol}_{\mathrm{Pt}}{ }^{-1} \cdot \mathrm{s}^{-1}$ as a $\mathrm{Pt} / \mathrm{Al}_{2} \mathrm{O}_{3}$ reference catalyst. The same research group has employed this material, platinum supported on niobium carbide $\left(\mathrm{Pt} / \mathrm{Nb}_{2} \mathrm{CT}_{\mathrm{x}}\right)$, as well as platinum supported on titanium carbide $\left(\mathrm{Pt} / \mathrm{Ti}_{3} \mathrm{C}_{2} \mathrm{~T}_{\mathrm{x}}\right)$ as catalysts for dehydrogenation of light alkanes. ${ }^{76}$ In this case, $\mathrm{Pt}-\mathrm{Ti}\left(\mathrm{Pt}_{3} \mathrm{Ti}\right)$ or $\mathrm{Pt}-\mathrm{Nb}\left(\mathrm{Pt}_{3} \mathrm{Nb}\right)$ alloy formation increased selectivity towards propene from 60 to $\sim 95 \%$ at $15 \%$ conversion through facilitating olefin desorption, which prevented secondary reactions.

The amount of literature on tuning MSI via support morphology for electro- or photocatalysis is limited. This can be rationalized by considering that this strategy often concerns metal oxides with limited conductivity. One exception is the work by Shi et al. ${ }^{77}$ on Au NPs supported on disordered $\mathrm{TiO}_{2}$ rich in oxygen vacancies, i.e., black titania. They found that the presence of intermediate energy levels within the band gap enhanced trapping of hot plasmonic electrons from gold and increased the photocurrent densities from 126 to $280 \mu \mathrm{A} \cdot \mathrm{cm}^{-1}$ with respect to $\mathrm{Au}$ on regular white $\mathrm{TiO}_{2}$, making photocatalytic water splitting more efficient. 


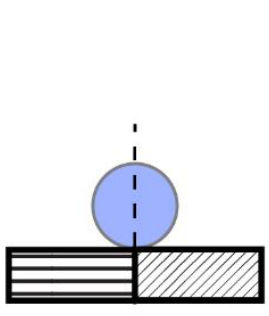

Compósition

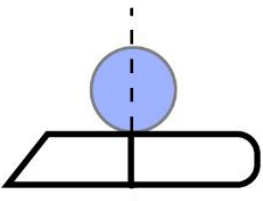

Morph'ology

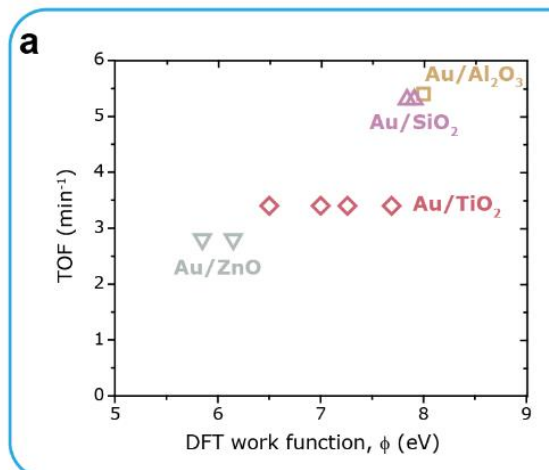

b
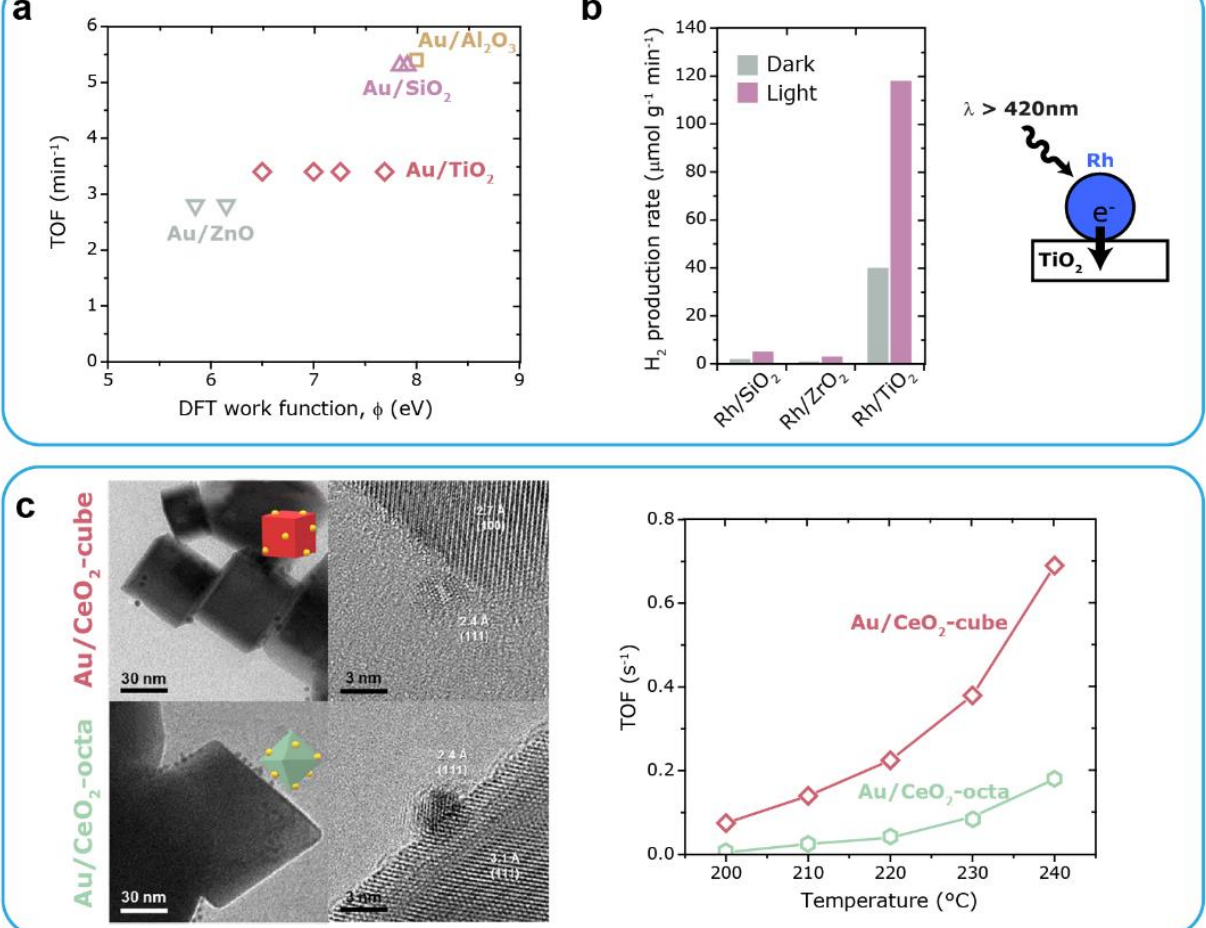

Figure 6.2. MSI tuning strategies involving modification of the support composition and morphology. a TOF for benzyl alcohol selective oxidation plotted against the work function of the supports, which is the inverse of the support's electron-donating ability to gold NPs. Multiple work function values were obtained for some supports, because these values were calculated for individual support crystal facets $\left(\right.$ ref. $^{53}$ ). b Methane steam reforming activity expressed as $\mathrm{H}_{2}$ production rate for Rh NPs supported on $\mathrm{SiO}_{2}, \mathrm{ZrO}_{2}$ and $\mathrm{TiO}_{2}$ with and without exposure to visible light $\left(\right.$ ref. ${ }^{54}$ ). c Use of different ceria morphologies (cubes or octahedra) as support for gold NPs and their effect on CO oxidation $\left(\right.$ ref. $\left.^{70}\right)$.

\subsubsection{Doping}

Doping of supports with elements of different valency mainly serves to regulate the electronic structure of the support (and metal NP) and typically results in enhanced or reduced electron donating properties.

Doping of carbons is an active field, especially for electrochemical applications such as batteries and supercapacitors and metal-free catalysis. When used as a support material for metallic nanoparticles, carbon can be doped with a variety of heteroatoms of which $\mathrm{N}$ has been most investigated. For a more comprehensive overview of how this strategy can be applied to obtain the desired reactivities, we refer to a recent review ${ }^{78}$. Here, we will cover the basics and highlight two studies which we believe illustrate the concept and possibilities.

$\mathrm{N}$-doping is often reported to increase the support-metal nanoparticle binding strength and therefore leads to smaller NPs and higher stabilities. ${ }^{79}$ Incorporating $\mathrm{N}$ in a carbon matrix can have various effects on its electronic structure, depending on the position of incorporation (e.g., pyrrolic, pyridinic or graphitic). Ning et al. ${ }^{80}$ investigated 
the electronic effect of different $\mathrm{N}$ species interacting with Pt nanoparticles of 2-4 $\mathrm{nm}$ and found that pyridinic $\mathrm{N}$ acted as an electron acceptor and graphitic $\mathrm{N}$ functioned as an electron donor for the Pt nanoparticles. The electron-enriched Pt NPs (in contact with graphitic N) gave the highest activity in electro-oxidation reactions of glycerol and formic acid. The high activity was partially related to an increased rate of CO removal, which otherwise poisons the catalyst. On the other hand, the electron-deficient Pt NPs (in contact with pyridinic N) showed the highest activity in ammonia borane hydrolysis. For both the electron-rich and-deficient NPs, the modified electronic structure was proposed to tune the Pt-reactant bonds, thereby optimizing reactant activation.

The application of carbon nitride with a stoichiometry of $\mathrm{C}_{2} \mathrm{~N}$ can be considered one of the most extreme cases of $\mathrm{N}$-doping of a support. ${ }^{81}$ These materials have a higher standard electrochemical potential than gold and are therefore termed noble carbons. ${ }^{82}$ This means that even gold is electron deficient, when supported on $\mathrm{C}_{2} \mathrm{~N}$, resulting in active materials for ammonia synthesis via electrocatalytic reduction of nitrogen. ${ }^{83}$ The positive effect of the $\mathrm{C}_{2} \mathrm{~N}$ support on catalysis was more pronounced when gold was dispersed as single atoms compared to nanoparticles, because of maximal MSI in the case of atomically dispersed metal.

Doping of a magnesium aluminate spinel support with Fe was investigated to improve the performance of a supported Ni catalyst for dry reforming of methane. ${ }^{84}$ During the reduction process, Ni catalyzed the reduction of Fe from the support most probably via Hspillover and, as a result, up to $50 \% \mathrm{Fe}$ was extracted from the support and an Ni-Fe alloy was formed at the NP surface. At an Fe/Ni ratio of $\sim 10$, carbon (coke) formation was inhibited. Interestingly, doping (Fe incorporated in the spinel) was more effective than surface modification (Fe on the surface of spinel), demonstrating the importance of the residual Fe ions left in the spinel structure of the $\mathrm{MgFe}_{\mathrm{x}} \mathrm{Al}_{2 \mathrm{x}} \mathrm{O}$ support.

Doping $\mathrm{Y}-\mathrm{Al}_{2} \mathrm{O}_{3}$ with $\mathrm{Mg}^{2+}$ ions prior to introduction of $\mathrm{Ni}$ partially prevented undesired mixed metal oxide formation during dry reforming of methane. ${ }^{85}$ This left more metallic Ni surface area available for catalysis and reduced deactivation. In addition, coke formation was suppressed, although that was mainly ascribed to the presence of small $(\sim 2 \mathrm{~nm})$ Ni particles. Recently, a related study on Ni-based catalysts for dry methane reforming was conducted, focusing on doping the $\mathrm{Al}_{2} \mathrm{O}_{3}$ with $\mathrm{La}^{3+}$ ions. ${ }^{86}$ The addition of $\mathrm{La}^{3+}$ had several effects on catalysis, such as increased activity at $\mathrm{La}^{3+}$ loadings between 2 and $10 \mathrm{wt} . \%$ and decreased selectivity towards coke formation, resulting in increasing stability with $\mathrm{La}^{3+}$ content. The positive influence of $\mathrm{La}^{3+}$ was ascribed to the introduction of basic sites and stronger MSI. It is quite possible that doping with $\mathrm{Mg}^{2+}$ and $\mathrm{La}^{3+}$ affects catalytic performance via the same mechanisms, as both form basic oxides, which may counteract the typically acidic nature of $\mathrm{Y}-\mathrm{Al}_{2} \mathrm{O}_{3}$.

Adding cationic or anionic dopants to reducible supports such as $\mathrm{TiO}_{2}$ can increase their conductivity, making them more attractive supports for electrocatalysis. When both type of dopants were applied simultaneously, more charge transfer to $3 \mathrm{~nm}$ Pt NPs was observed. ${ }^{87}$ This resulted in 3 to 5 times higher Pt mass specific activity in electrocatalytic oxygen reduction than obtained with only one dopant (Figure 6.3a). Similarly, an increase in charge transfer from $\mathrm{Fe}^{3+}$-doped $\mathrm{ZnO}$ to Pt NPs reportedly led to a 5-fold increase in TOF 
in CO oxidation..$^{88}$ Other examples of dopants that affect oxygen vacancies or charge transfer in reducible supports examples are available as well. ${ }^{89-91}$ Furthermore, Schumann et al. $^{92}$ found that dopants can stabilize the metal oxide by changing their reducibility. Trivalent dopants $\left(\mathrm{Al}^{3+}, \mathrm{Ga}^{3+}\right)$ increased the conductivity of the support and activity in reverse water-gas shift and methanol synthesis, whereas divalent $\mathrm{Mg}^{2+}$ lowered the activity.

\subsubsection{Surface modification}

We distinguish two types of surface modification: introduction of functional groups and (complete) coating of the surface with another phase up to the point that the surface properties are completely dictated by the coating material.

Oxygen- or nitrogen-containing functional groups are often introduced on carbon supports to regulate MSI. The basic concept is that these functionalities act as anchoring sites for the metal NPs. That just anchoring NPs is not a complete picture was recognized for example by two groups working on surface functionalization of CNT as support for Co catalysts for the Fischer-Tropsch synthesis. ${ }^{93-95}$ Eschemann et al. ${ }^{95}$ reported substantially higher TOF for Co supported on pristine carbon nanotubes (CNT) compared to surfaceoxidized CNT. The authors proposed an increased fraction of more active hcp Co on pristine $\mathrm{CNT}$ as the cause of these differences. This hypothesis can be strengthened by earlier literature, as Co was previously shown to form of an hep phase on graphite (0001). ${ }^{96}$ In addition, several other studies showed that the role of functional groups was not limited to anchoring of NPs in hydrogenation ${ }^{97,98}$ and oxidation ${ }^{99}$ of organic compounds. Charge transfer between the functionalized support and noble metal nanoparticles substantially altered catalytic performance (Figure 6.3b).

Metal oxides can also be functionalized to facilitate metal NP binding using, e.g., amine or thiol groups to anchor metal NPs to a support. These functional groups can affect the activity and selectivity of catalysts supported on irreducible oxides ${ }^{100,101}$, but this is not very common. Often the functional groups do not change the intrinsic activity of the catalysts and rather facilitate binding to the support and can impart greater stability to the catalysts. ${ }^{102}$

Surface coating can be applied to the support via precursor decomposition (glucose, organometallic precursors, etc.) or atomic-layer deposition. For example, coating the surface of a nanostructured $\operatorname{In}_{2} \mathrm{O}_{3}$ with carbon made this support more suitable for photocatalytic $\mathrm{CO}_{2}$ reduction using Pt as the metal. ${ }^{103}$ The Pt NPs were the catalytically active sites and the carbon increased absorption of visible light through lowering of the bandgap, lowered electron-hole recombination, suppressed $\mathrm{H}_{2}$ production from protons, and facilitated $\mathrm{CO}_{2}$ adsorption. Through all these support functions, the production rate of $\mathrm{CO}$ increased from 27 to $127 \mu \mathrm{mol}_{\mathrm{CO}} \cdot \mathrm{h}^{-1}$ and the production of methane increased from 4 to

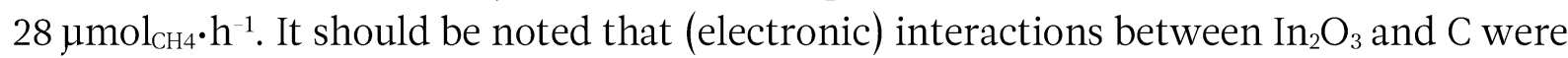
present, so the composite material displayed the combined properties of both materials.

Prieto et al. ${ }^{104}$ utilized coatings to isolate the intrinsic properties of different metal oxide supports from other factors such as support morphology and verified the effect of these properties on the performance of $\mathrm{Ru}$-promoted Co catalysts. The authors found a correlation between the Lewis acidity of the metal oxide surface layer and the catalytic 
activity and selectivity in the Fischer-Tropsch synthesis (Figure 6.3c). These studies show that a surface layer can be applied to effectively replace the surface properties of the original support with that of the coating.

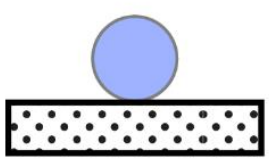

Doping

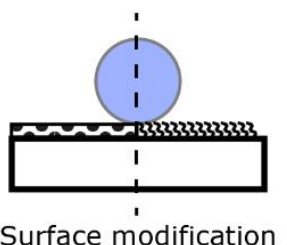

Surface modification
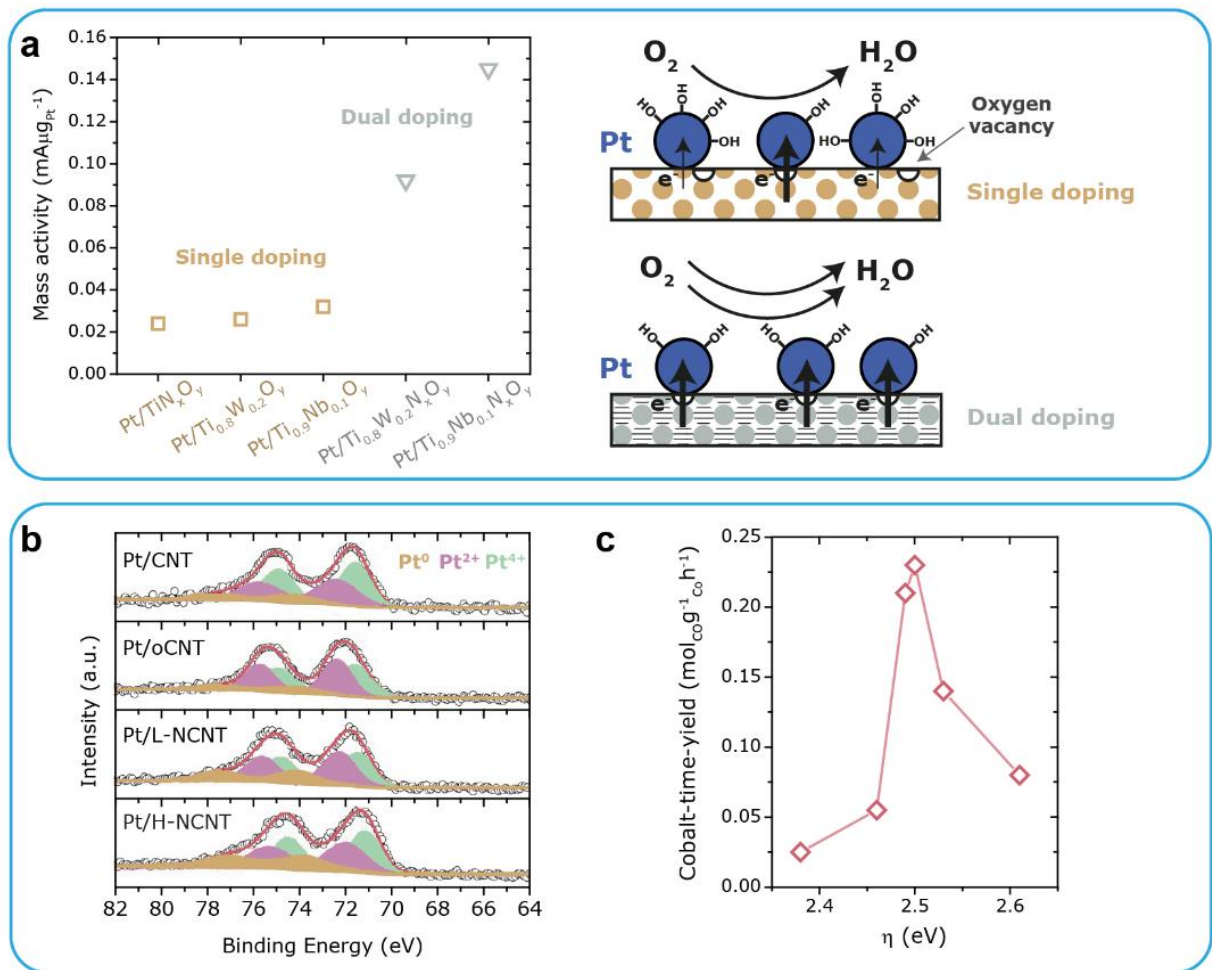

C

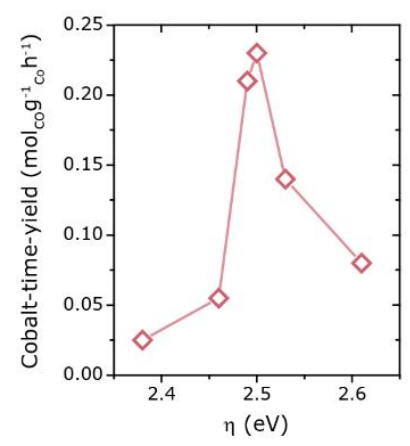

Figure 6.3. MSI tuning strategies based on doping and surface modification of the support. a Chart comparing the electrochemical performance in the oxygen reduction reaction of Pt on various single- and dual-doped titania supports and schematic representation of the degree of charge transfer from the support to the Pt NPs depending on the kind of doping, as depicted by the thickness of the arrows (ref. $\left.{ }^{87}\right)$. $\mathbf{b}$ XPS spectra of Pt $4 \mathrm{f}$ for Pt supported on carbon nanotubes (CNT) with various pre-treatments: pristine $(\mathrm{Pt} / \mathrm{CNT})$, oxidized $(\mathrm{Pt} / \mathrm{oCNT})$, ammonia treated $(\mathrm{Pt} / \mathrm{L}-\mathrm{NCNT})$ or high nitrogen content $(\mathrm{Pt} / \mathrm{H}-\mathrm{NCNT})$. The spectra show changes in the oxidation state of $\mathrm{Pt}$ depending on the CNT surface modification ( $\left(\right.$ ef $\left.^{9}{ }^{98}\right)$. c Cobalt-normalized activities at pseudo-steady state for the Fischer-Tropsch synthesis reaction as a function of the acid-base character of the metal oxide surface layer inferred from the UV-vis band shift of a probe molecule $(\eta)$ due to charge transfer $\left(\right.$ ref. $\left.{ }^{104}\right)$.

\subsection{Metal nanoparticles}

The second category of MSI tuning focusses mainly on the metal NPs. Of the three stages for MSI tuning (support, metal NP, treatment), this is least reported, yet it does provide interesting opportunities. In this section, we will discuss recent examples of how modification of the size or composition of the nanoparticles affects MSI and hence catalytic performance. 


\subsubsection{Size}

MSI can have a strong influence on the size of metal NPs, clusters and single atoms during catalysis and we refer to a recent review for a detailed discussion on this topic. ${ }^{105}$ Focusing on metal NPs, the vast majority of literature on MSI tuning deals with NPs $<5 \mathrm{~nm}$. Most probably because the effect of the support on the metal NPs is attenuated for larger NPs because of reduced impact of charge transfer and relatively less metallic surface area in intimate contact with the support, i.e., less interface sites. NP size has the most pronounced influence on purely electronic MSI effects, which are short range, as Lykhach et al. ${ }^{106}$ showed for 1.0-1.5 nm Pt NPs with a partial positive charge of 0.11 per Pt atom in $\mathrm{Pt} / \mathrm{CeO}_{2}$ catalyst (Figure 6.4a). They observed using resonant photoemission spectroscopy on 2D model systems that the partial positive charge per Pt atom decayed for larger particles. Furthermore, the overall charge transfer was limited by the support $\left(17 \%\right.$ surface $\mathrm{Ce}^{3+}$ seems the limit for $\mathrm{CeO}_{2}$ reduction). The need for small NPs to induce effective electronic MSI is also reflected in literature where most reports on catalytic performance relate to particles smaller than $5 \mathrm{~nm}$ (Figure 6.7b). Conversely, this also means that it is unlikely that electronic MSI plays a significant role in large $(>2 \mathrm{~nm})$ NPs, unless the NPs are encapsulated by the support in which case charge transfer could still occur to all surface sites.

Guo et al. ${ }^{107}$ investigated the effect of $\mathrm{Ru} \mathrm{NP}$ size on the performance of $\mathrm{Ru} / \mathrm{CeO}_{2}$ in $\mathrm{CO}_{2}$ hydrogenation varying between single atoms, $1 \mathrm{~nm}$ clusters and $4 \mathrm{~nm} \mathrm{NP}$. With increasing NP size, the electronic MSI interaction decreased which facilitated $\mathrm{CO}_{2}$ activation and increased reactivity (Figure 6.4b). At the same time, H-spillover increased with NP size, hindering $\mathrm{H}_{2} \mathrm{O}$ removal and decreasing activity. Competition between both effects makes the $1 \mathrm{~nm}$ clusters (intermediate size) the most active system with a TOF about $50 \%$ higher than single atoms and one order of magnitude higher than NPs. Interestingly, the selectivity to $\mathrm{CH}_{4}$ was $100 \%$ for all sizes. This is in contradiction to a recent report by Yan et al. ${ }^{108}$, who observed a $\mathrm{Ru} \mathrm{NP}$ size-dependent selectivity in $\mathrm{CO}_{2}$ hydrogenation on $\mathrm{Y}^{-}$ $\mathrm{Al}_{2} \mathrm{O}_{3}$. CO formation via reverse WGS was favored for $\mathrm{Ru}$ clusters while the selectivity shifted to $\mathrm{CH}_{4}$ for larger NPs. One possible explanation for the different observations could be the nature of the supports that were employed $\left(\mathrm{CeO}_{2}\right.$ and $\left.\gamma-\mathrm{Al}_{2} \mathrm{O}_{3}\right)$, because the support was involved in the reaction in both cases. Notably, in $\mathrm{Ru} / \mathrm{\gamma}-\mathrm{Al}_{2} \mathrm{O}_{3}$, the interface oxygen $(\mathrm{Ru}-\mathrm{O}-\mathrm{Al})$ was shown via isotope labelling to exchange oxygen atoms with $\mathrm{CO}_{2}$ during the reaction.

\subsubsection{Composition}

The metal distribution in an NP depends to a large extent on the strength of the bonds between the metal atoms and between metal atoms and support. Theoretical calculations. ${ }^{109}$ on $\mathrm{Au}-\mathrm{Rh}$ and $\mathrm{Pd}$-Ir nanoparticles supported on titania showed that the surface binding strength of $\mathrm{Rh}$ or Ir atoms to $\mathrm{TiO}_{2}(110)$ is greater than for $\mathrm{Pd}$ or $\mathrm{Au}$ to the support, due to stronger $\mathrm{Rh}-\mathrm{O}$ or Ir-O interactions than $\mathrm{Au}-\mathrm{O}$ or Pd-O. Therefore, $\mathrm{Rh}$ or Ir segregated towards $\mathrm{TiO}_{2}$. This has been experimentally observed for $\mathrm{Au}-\mathrm{Rh}$ nanoparticles supported on titania nanorods ${ }^{110}$ (Figure 6.4c) and employed for tetralin hydrogenation in the presence of $\mathrm{H}_{2} \mathrm{~S}^{111}$. The $\mathrm{Au} / \mathrm{Rh} / \mathrm{TiO}_{2}$ stacking configuration had a detrimental effect on the initial hydrogenation activity. Eventually, $\mathrm{Au} / \mathrm{Rh} / \mathrm{TiO}_{2}$ was more stable due to the stabilizing effect of gold against $\mathrm{H}_{2} \mathrm{~S}$ poisoning of Rh. Likewise, a 
segregation of iridium from $\mathrm{Au}$-Ir nanoparticles towards $\mathrm{TiO}_{2}$ has been experimentally observed, resulting in higher resistance towards sintering than $\mathrm{Au} / \mathrm{TiO}_{2}$ during thermal treatments. ${ }^{112}$

For $\mathrm{Au}-\mathrm{Cu}$ on $\mathrm{Al}_{2} \mathrm{O}_{3}$ and $\mathrm{SiO}_{2}$ the NPs de-alloyed under oxidizing conditions on both supports to form $\mathrm{Au}$ NPs and a $\mathrm{CuO}_{\mathrm{x}}$ phase. However, on $\mathrm{Al}_{2} \mathrm{O}_{3}, \mathrm{CuO}_{\mathrm{x}}$ formed separate patches on the support, which prevented the creation of active $\mathrm{Au}-\mathrm{CuO}_{\mathrm{x}}$ interfacial sites present on $\mathrm{SiO}_{2} \cdot{ }^{113}$ Consequently, $\mathrm{Au}-\mathrm{CuO} / \mathrm{SiO}_{2}$ approached $100 \% \mathrm{CO}$ conversion while $\mathrm{Au}-\mathrm{CuO} / \mathrm{Al}_{2} \mathrm{O}_{3}$ converted $11 \%$ of the $\mathrm{CO}$ at $150{ }^{\circ} \mathrm{C}$.

Seemala et al. ${ }^{114}$ studied $\mathrm{Cu}-\mathrm{Ni}$ nanoparticles supported on either $\theta$-alumina or titania and observed $\mathrm{TiO}_{2}$-induced metal segregation upon reduction using $\mathrm{H}_{2}$, during which $\mathrm{Cu}$ enriched the NP surface while Ni segregated to the NP-support interface. This arrangement was explained by a stronger interaction between nickel and titania than copper and titania. The $\mathrm{Cu}-\mathrm{Ni} / \mathrm{TiO}_{2}$ catalyst showed high activity in furfural hydrodeoxygenation, high selectivity to methyl furan and good recyclability, outperforming the $\mathrm{Cu}$-Ni supported on alumina and the titania-supported monometallic catalysts. A similar phenomenon was observed in the case of Ni-Pt supported on mesoporous silica (SBA-15). ${ }^{115}$ Here, a strong nickel-support interaction, most likely generated during metal precursor deposition on silica at basic conditions, led to the formation of a $\mathrm{Pt}-\mathrm{rich}\left(\mathrm{Pt}_{3} \mathrm{Ni}\right)$ intermetallic compound thus favoring the formation of active $\mathrm{Pt}-\mathrm{Ni}$ bonds rather than $\mathrm{Ni}-\mathrm{Ni}$. This in turn increased the selectivity of $\mathrm{CO}_{2}$ reduction to $\mathrm{CO}$ instead of $\mathrm{CH}_{4}$, as the latter product is typical for $\mathrm{Ni}$ based catalysts.

Divins et al. ${ }^{116}$ showed that ceria as support influenced the oxidation state and metal arrangement of $\mathrm{Rh}-\mathrm{Pd}$ nanoparticles under different atmospheres using NAP-XPS at 0.05 mbar. Upon reduction at $573 \mathrm{~K}, \mathrm{Rh}-\mathrm{Pd} / \mathrm{CeO}_{2}$ and non-supported $\mathrm{Rh}-\mathrm{Pd}$ nanoparticles showed segregation of palladium to the surface. However, at conditions close to ethanol steam reforming the metals in the non-supported nanoparticles re-arranged back to a uniform distribution, whereas the $\mathrm{CeO}_{2}$-supported ones maintained the Pd-rich surface. Because the $\mathrm{Rh}-\mathrm{CeO}_{2}$ interaction was stronger, the core-shell structure was maintained. Furthermore, the $\mathrm{CeO}_{2}$-supported nanoparticles presented higher oxidation states, especially at the outermost layers due to $\mathrm{H}_{2} \mathrm{O}$ activation at the $\mathrm{CeO}_{2}$ surface. The high $\mathrm{H}_{2}$ selectivity of the supported catalyst, compared to the non-supported nanoparticles, was derived from promotion of WGS and methane steam reforming.

Gubó et al. ${ }^{117}$ showed that an increase of the relative $\mathrm{Au}$ concentration in $\mathrm{Au}-\mathrm{Pd}$ nanoparticles supported on rutile $\mathrm{TiO}_{2}$ prevented the encapsulation of the nanoparticles by $\mathrm{TiO}_{\mathrm{x}}$. The nanoparticles formed a bimetallic core with an $\mathrm{Au}$ enriched shell and became less prone to SMSI due to the lower surface free energy of Au.

Organic ligands coordinated to the surface of metal NPs can affect MSI, e.g. by acting as a carbon-precursor. Zhan et al. ${ }^{118}$ placed pre-formed Au colloids, capped with oleylamine ligands, on $\mathrm{TiO}_{2}$. The ligands were decomposed using an $\mathrm{N}_{2}$ treatment to form a carbon shell, which protected Au NPs from sintering. The higher overall CO oxidation activity of the $\mathrm{N}_{2}$-treated sample was ascribed to smaller NP size. Gao et al. ${ }^{119}$ coimpregnated a nickel precursor and ligands on $\mathrm{SiO}_{2}$ and obtained different NP sizes and stability during dry reforming, depending on the ligands (oleic acid, oleylamine or a 
mixture of both). The smaller NP size led to doubling of the $\mathrm{CH}_{4}$ conversion, while the surface-specific activity remained unchanged $\left(\sim 2.5 \mathrm{~s}^{-1}\right)$.
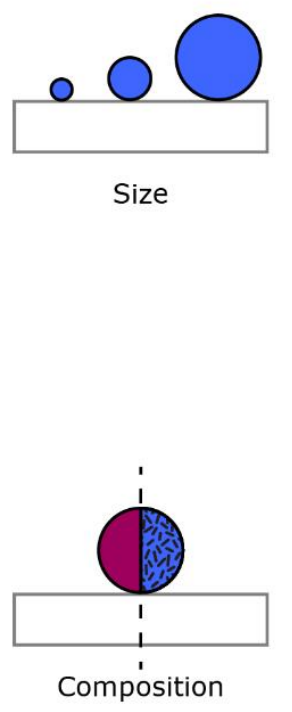

b

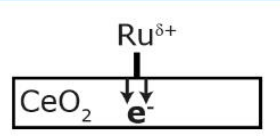

TOF

$4.6 \cdot 10^{-3} \mathrm{~s}^{-1}$

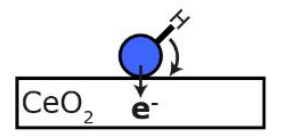

$7.4 \cdot 10^{-3} \mathrm{~s}^{-1}$

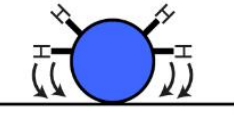

$\mathrm{CeO}_{2}$
$0.5 \cdot 10^{-3} \mathrm{~s}^{-1}$
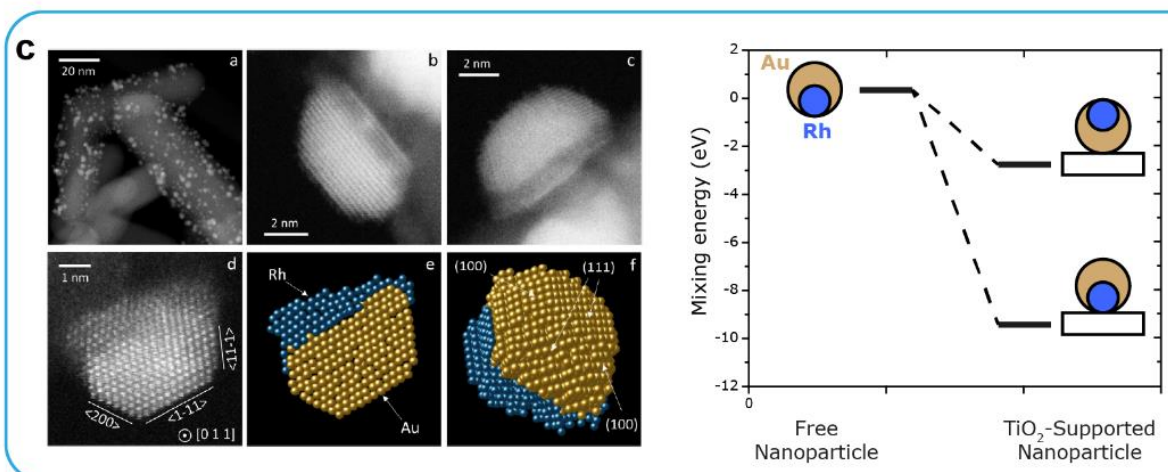

Figure 6.4. MSI tuning strategies involving alterations to the metal nanoparticle size or composition. a Average charge per Pt atom to the ceria support as a function of the Pt particle size (ref. $\left.{ }^{106}\right)$. $\mathbf{b}$ Schematic representation of the balance between charge transfer and $\mathrm{H}$-spillover for $\mathrm{Ru}$ of different sizes (atoms, $1 \mathrm{~nm}$ nanoclusters and $4 \mathrm{~nm}$ nanoparticles) supported on ceria and its effect on the TOF in $\mathrm{CO}_{2}$ methanation (ref. ${ }^{107}$ ). $\mathrm{c}$ HAADF images and model reconstruction showing Rh segregation to the $\mathrm{TiO}_{2}$ support for a reduced $\mathrm{AuRh} / \mathrm{TiO}_{2}$ sample and the energy scheme for two binding configurations of AuRh clusters to $\mathrm{TiO}_{2}$ derived from DFT. Calculations point to Rh segregation to the support as the most favorable configuration $\left(\right.$ ref. $\left.{ }^{110}\right)$.

\subsection{Treatments}

We define treatments as any action to modify MSI that is performed on the metal-support composite material prior to catalysis. This has mainly been investigated in the context of tuning MSI of metals with reducible supports, i.e., SMSI.

\subsubsection{Thermal treatment}

Tang et al. investigated MSI for various group 11 metals on $\mathrm{TiO}_{2}$ and for $\mathrm{Au}$ supported on various reducible oxidic supports. ${ }^{120}$ They observed that metal NP overgrowth under reducing conditions started at $200{ }^{\circ} \mathrm{C}$ and was most pronounced at $400-500{ }^{\circ} \mathrm{C}$. The overgrowth blocked the accessible metallic surface area and thereby quenched the reactivity for $\mathrm{CO}$ oxidation, in line with earlier literature on group 8-10 metals on reducible 
oxides. Furthermore, a simultaneous charge transfer from the support to Au was observed by XPS, resulting in an electron rich Au phase. These effects could be reversed and the reactivity could be restored by an oxidative treatment at $400{ }^{\circ} \mathrm{C}$. Interestingly, the same authors also observed similar effects for Au NPs supported on a phosphate hydroxyapatite, yet with the reverse response to oxidizing and reducing conditions ${ }^{121}$; now oxidation at $300{ }^{\circ} \mathrm{C}$ and higher resulted in the support encapsulating the Au NPs and lowering the CO oxidation activity, which could be reversed by a reductive treatment at $500{ }^{\circ} \mathrm{C}$. It was recently suggested that loss of $\mathrm{OH}$ groups at high temperature gave rise to this type of interaction and the phenomenon was also observed on group 10 metals supported on $\mathrm{ZnO}-$ nanorods. ${ }^{122}$ Wang et al. ${ }^{123}$ even avoided the necessity for reducing or oxidizing atmospheres altogether by exploiting the thermally-induced transition of a layered double hydroxide to the corresponding oxide in an inert atmosphere, possibly also caused by loss of OH groups. This induced a similar encapsulation of Au NPs with lowered CO oxidation activity.

Changes in activity and product selectivities during $\mathrm{CO}$ hydrogenation using $\mathrm{Ni} / \mathrm{Nb}_{2} \mathrm{O}_{5}$ were reported after different reduction temperatures $\left(250-450{ }^{\circ} \mathrm{C}\right)$ and related to SMSI effects. ${ }^{124}$ An intermediate reduction temperature $\left(350{ }^{\circ} \mathrm{C}\right)$ showed the highest activity $\left(3.9 \cdot 10^{-5} \mathrm{~mol}_{\mathrm{CO}} \cdot \mathrm{g}_{\mathrm{Ni}^{-1}} \cdot \mathrm{s}^{-1}\right)$ and $\mathrm{C}_{5_{+}}$-selectivity $(55 \mathrm{wt} . \%)$. Lower reduction temperature resulted in severe deactivation due to particle growth via nickel tetracarbonyl formation and a lower final activity $\left(2.2 \cdot 10^{-5} \mathrm{~mol}_{\mathrm{CO}} \cdot \mathrm{g}_{\mathrm{Ni}}{ }^{-1} \cdot \mathrm{s}^{-1}\right)$. On the other hand, a higher reduction temperature $\left(450{ }^{\circ} \mathrm{C}\right)$ displayed stable performance but with a medium activity $\left(2.7 \cdot 10^{-5}\right.$ $\mathrm{mol}_{\mathrm{CO}} \cdot \mathrm{g}_{\mathrm{Ni}}^{-1} \cdot \mathrm{s}^{-1}$ ) and a selectivity shift to shorter hydrocarbon products, probably due to blockage of active sites by $\mathrm{NbO}_{x}$ suboxides. Similarly, in the case of $\mathrm{Ni} / \mathrm{TiO}_{2}$ catalysts for WGS, the coverage of $\mathrm{Ni}$ nanoparticles by $\mathrm{TiO}_{\mathrm{x}}$ could be tuned via reduction temperatures between 400 and $600{ }^{\circ} \mathrm{C}$ (Figure 6.5a). ${ }^{125}$ The metal-support interface was maximized by starting from an NiTi-layered double hydroxide precursor. Charge transfer to interfacial $\mathrm{Ni}$, coverage of $\mathrm{Ni} \mathrm{NP}$ by suboxides and the number of oxygen vacancies in $\mathrm{TiO}_{\mathrm{x}}$ all increased with higher reduction temperature. Moderate MSI after reduction at $450{ }^{\circ} \mathrm{C}$ showed the highest catalytic activity with a TOF of $3.8 \mathrm{~s}^{-1}$, about two times more active than after reduction at 400,500 or $600{ }^{\circ} \mathrm{C}$. The high activity was attributed to the interplay between interfacial $\mathrm{Ni}^{--}$and oxygen vacancies. Another example of the influence of temperature on support reduction was reported for $\mathrm{Pd} / \mathrm{TiO}_{2}$ in formaldehyde oxidation. ${ }^{126}$ When reduced at $450{ }^{\circ} \mathrm{C}$, more oxygen vacancies were generated, which in turn aided $\mathrm{O}_{2}$ activation and $\mathrm{H}_{2} \mathrm{O}$ dissociation to form $\mathrm{OH}$ groups and increased the formaldehyde oxidation activity 7.5fold. These species directly decomposed formaldehyde without the slow formation of $\mathrm{CO}$ as intermediate, as observed after reduction at $300{ }^{\circ} \mathrm{C}$.

An intermetallic nickel silicide catalyst obtained by reacting a nickel complex with silica via pyrolysis at high temperatures has been shown to be highly active for chemoselective hydrogenation of a wide variety of compounds (e.g., nitroarenes, carbonyls, nitriles, N-heterocycles and unsaturated carbon-carbon bonds). ${ }^{127}$ The researchers found that the formation of this versatile catalyst strongly depended on the pyrolysis temperature, the nitrogen-doped carbon species from the ligand for $\mathrm{Si}-\mathrm{O}$ bond reduction and the silica specific surface area. A pyrolysis temperature of $600{ }^{\circ} \mathrm{C}$ resulted in metallic nickel supported on silica and upon increasing the pyrolysis temperature to $800{ }^{\circ} \mathrm{C}$ 
a $\mathrm{Ni}_{17} \mathrm{Si}_{3}$ phase was observed. The most active catalyst was obtained at $1000{ }^{\circ} \mathrm{C}$ with siliconenriched phases $\mathrm{Ni}_{31} \mathrm{Si}_{12}$ and $\mathrm{Ni}_{2} \mathrm{Si}$. Additionally, coverage of metal nanoparticles by support suboxides generated at elevated temperature can steer hydrogenation selectivity, as summarized before ${ }^{128}$.

\subsubsection{Reducing agent}

The extent of MSI and in particular partial support reduction to form suboxides and oxygen vacancies depend on the overall severity of the reduction treatment. In 2014, Rui et al. ${ }^{129}$ reported that the SMSI state in $\mathrm{Pt} / \mathrm{TiO}_{2}$ could be induced at room temperature during a liquid phase reduction process using $\mathrm{NaBH}_{4}$ or formaldehyde as reducing agent. The SMSI state, characterized by suppressed CO chemisorption, negatively charged Pt NPs, and oxygen vacancies formation, was beneficial for toluene oxidation activity, which decreased in the order $\mathrm{NaBH}_{4}>$ formaldehyde $>\mathrm{H}_{2}$. Furthermore, the MSI effect was stronger on anatase than on rutile or P25, again highlighting the importance of the support morphology as discussed in section 3.2.

Few systematic studies focusing on the effect of the reducing agent on MSI have been published in the meantime. A recent example includes the use of reducing agents with different reduction strengths to determine the rate of Pt-precursor reduction, which in turn regulated the association of NPs to different types of $\mathrm{N}$-functionalities in N-doped carbon nanotubes (see section 3.3) ${ }^{80}$ As a second recent example, Gänzler et al. ${ }^{130,131}$ showed that the $\mathrm{CO}$ oxidation activity of Pt NPs supported on a $\mathrm{CeO}_{2} / \mathrm{Al}_{2} \mathrm{O}_{3}$ composite support strongly varied as a function of the reducing agent, following the order $\mathrm{CO}>\mathrm{H}_{2}>\mathrm{C}_{3} \mathrm{H}_{6}$ (Figure 6.5c) The effect of the reducing agent was ascribed to its ability to partially reduce the support. Additionally, MSI and catalytic performance could be further tuned by reduction-oxidation cycles (see next section).

\subsubsection{Reduction-oxidation cycles}

Reduction-oxidation ( $\mathrm{RO}$ ) cycles have been intensively investigated, mainly to regulate the metal NP size through re-dispersion, but also to affect MSI in various ways. Here, we will highlight several possibilities of RO for MSI tuning that were recently published.

Besides different reducing agents, Gänzler et al. ${ }^{130,131}$ applied RO cycles on a CO oxidation catalyst consisting of $\mathrm{Pt} / \mathrm{CeO}_{2} / \mathrm{Al}_{2} \mathrm{O}_{3}$ in order to control both the Pt NP size and oxidation state, which in turn affected the reduction of $\mathrm{CeO}_{2}$. Metallic Pt NPs were necessary to reduce $\mathrm{CeO}_{2}$ at low temperature and the support had a crucial role in $\mathrm{CO}$ oxidation, as discussed before. $1.4 \mathrm{~nm}$ Pt NPs were identified as the optimal size, because at this size most interfacial sites were available while maintaining Pt in the active metallic state. Further RO cycles induced Pt NP growth and reduced the CO oxidation activity

Alternatively, Freakley et al. ${ }^{132}$ employed the reduction step of an RO cycle on a Pd$\mathrm{Sn} / \mathrm{TiO}_{2}$ catalyst to selectively modify small $\mathrm{Pd}$ NPs that otherwise gave rise to side reactions. Surprisingly, the NPs were not covered by $\mathrm{TiO}_{x}$ species, but by $\mathrm{SnO}_{x}$ species, as evidenced by STEM-EELS and similarly with a Pd-Sn system supported on irreducible $\mathrm{SiO}_{2}$. The final oxidation step was necessary to generate $\mathrm{Pd}^{2+}$ ions on the larger NPs, which were less active for side reactions than metallic $\mathrm{Pd}$. The investigated reaction was the direct synthesis of $\mathrm{H}_{2} \mathrm{O}_{2}$ from $\mathrm{O}_{2}$ and $\mathrm{H}_{2}$ and the selectivity is a balance between $\mathrm{H}_{2} \mathrm{O}_{2}$ production 
rate (hydrogenation of $\mathrm{O}_{2}$ ) and $\mathrm{H}_{2} \mathrm{O}_{2}$ decomposition rate. By covering the small NPs and having a $\mathrm{Pd}^{2+}$ containing surface on the larger NPs, the $\mathrm{H}_{2} \mathrm{O}_{2}$ decomposition was almost completely suppressed, resulting in hydrogen selectivities towards $\mathrm{H}_{2} \mathrm{O}_{2}$ of $95 \%$.

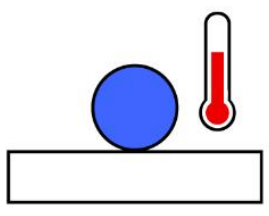

Thermal
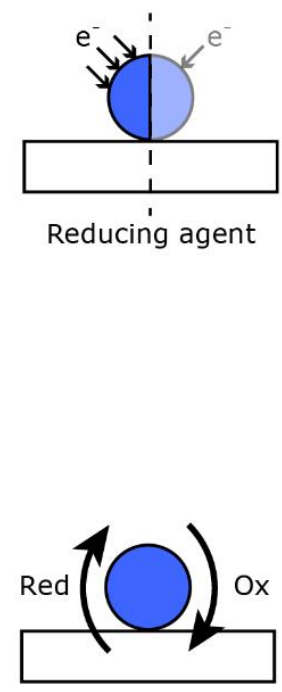

Reduction-oxidation cycles

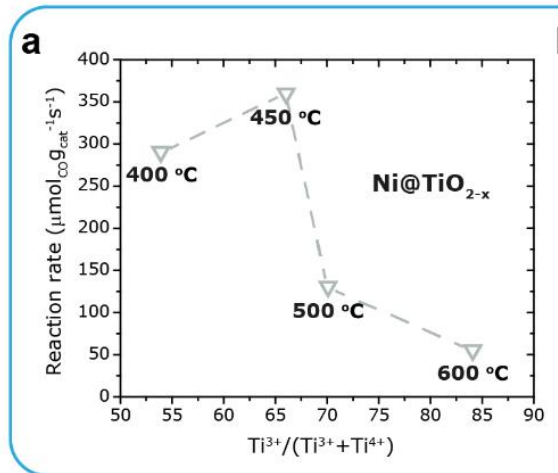

b
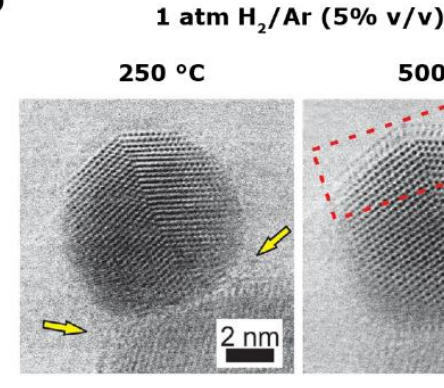

$500{ }^{\circ} \mathrm{C}$

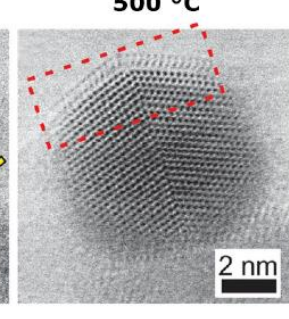

$2 \mathrm{~nm}$
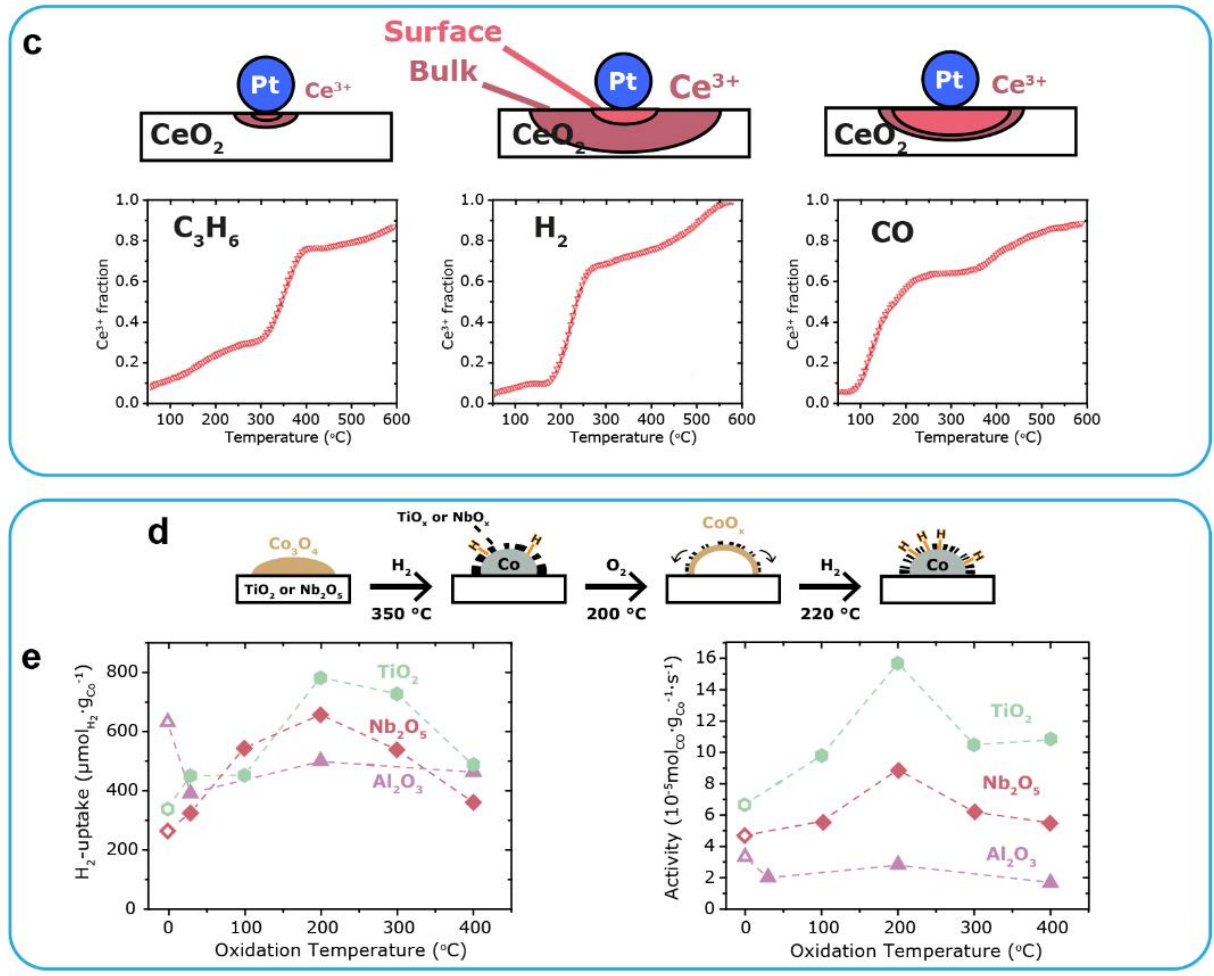

Figure 6.5. MSI tuning strategies involving treatments of supported metal catalysts based on temperature, reducing agent or reduction-oxidation cycles. a The influence of reduction temperature of $\mathrm{Ni} @ \mathrm{TiO}_{\mathrm{x}}$ on the $\mathrm{Ti}^{3+}$ to $\mathrm{Ti}^{4+}$ ratio and WGS reaction rate $\left(\right.$ ref. $\left.{ }^{225}\right)$. $\mathbf{b}$ In-situ observation of a $\mathrm{TiO}_{\mathrm{x}}$ overlayer on a Pd NP under reducing conditions at 5 vol. $\% \mathrm{H}_{2}$ in $\mathrm{Ar}, 1$ bar, 250 and $500{ }^{\circ} \mathrm{C}$ (ref. $\left.{ }^{48}\right)$. c Support reduction using different reducing agents $\left(\mathrm{C}_{3} \mathrm{H}_{6}, \mathrm{H}_{2}\right.$ or $\left.\mathrm{CO}\right)$ for $\mathrm{PtO}_{\mathrm{x}}$ on ceria in temperature-programmed experiments, as deduced from the fraction of $\mathrm{Ce}^{3+}$ obtained by Ce L3edge XANES spectra (ref. ${ }^{131}$ ). $\mathbf{d}$ Schematic representation of a reduction-oxidation-reduction (ROR) treatment of cobalt supported on reducible supports. e The effect of different oxidation (O) temperatures in the ROR procedure on the hydrogen uptake in chemisorption experiments and Fischer-Tropsch synthesis activity of $\mathrm{Co} / \mathrm{TiO}_{2}$ (green hexagons), $\mathrm{Co} / \mathrm{Nb}_{2} \mathrm{O}_{5}$ (red diamonds) and $\mathrm{Co} / \mathrm{Al}_{2} \mathrm{O}_{3}$ (violet triangles). Singly reduced samples are indicated by open symbols (ref. ${ }^{133}$ ). 
It is well established that the SMSI state is typically generated under reducing conditions and that the SMSI state can be reversed by an oxidative treatment. ${ }^{48}$ In an earlier publication ${ }^{133}$, we applied this principle to enhance the FT activity of $\mathrm{Co} / \mathrm{TiO}_{2}$ and $\mathrm{Co} / \mathrm{Nb}_{2} \mathrm{O}_{5}$ catalysts via a reduction-oxidation-reduction (ROR) process (Figure 6.5d-e). The first reduction at $350{ }^{\circ} \mathrm{C}$ fully reduced the cobalt oxide to metallic cobalt and simultaneously induced the SMSI state. Oxidation at $200{ }^{\circ} \mathrm{C}$ modified the suboxides, but did not oxidize the cobalt fully to $\mathrm{Co}_{3} \mathrm{O}_{4}$. Consequently, the second reduction could be performed at $220{ }^{\circ} \mathrm{C}$ only, which resulted in less suboxide coverage and hence twice the accessible metallic surface area, as compared to the samples reduced at $350{ }^{\circ} \mathrm{C}$. The FT activity increased proportionally to the metallic surface area, implying that the number of active sites increased and that the TOF and thus the nature of the active sites remained unchanged.

\subsubsection{Overlayer deposition}

As-prepared catalysts containing NPs on a support can be covered with an overlayer of another compound and its most basic function is to stabilize the NPs via immobilization, as was the case for a carbon ${ }^{134}$ and $\mathrm{SiO}_{2}{ }^{135}$ overlayer. In addition, in a 2D model study, a Pt(111) surface was covered with a thin layer of $\mathrm{TiO}_{\mathrm{x}}$ to mimic an SMSI state. ${ }^{136}$ Compared to the clean Pt(111) surface, the $\mathrm{TiO}_{\mathrm{x}}$ species increased $\mathrm{CO}$ oxidation activity and the effect was assigned to the formation of interfacial perimeter sites. Furthermore, Weng et al. ${ }^{137}$ deposited $\mathrm{SiO}_{2}$ over $\mathrm{Pt} / \mathrm{Al}_{2} \mathrm{O}_{3}$ and observed the formation of Brønsted acid $\mathrm{Si}-\mathrm{O}-\mathrm{Al}$ sites. The $\mathrm{SiO}_{2}$ overlayer decreased activity in cinnamaldehyde hydrogenation, possibly via site blockage by $\mathrm{SiO}_{2}$, while at the same time, selectivity towards cinnamyl alcohol increased from $25-30 \%$ to $85 \%$.

Overlayers can also be applied to create specific interfacial sites, such as $\mathrm{Cu}-\mathrm{ZrO}_{2}$ in a $\mathrm{Cu} / \mathrm{SiO}_{2}$ catalyst. ${ }^{138}$ These interface sites enhanced the intrinsic activity for ethyl acetate formation from ethanol and for methanol synthesis from $\mathrm{CO}_{2}$ by one order of magnitude. Furthermore, Moon et al. ${ }^{139}$ applied an overlayer of various reducible oxides to a $\mathrm{Pt} / \mathrm{SiO}_{2}$ catalyst and investigated its effect on $\mathrm{CO}$ oxidation. The activity followed the order $\mathrm{TiO}_{2}>\mathrm{CeO}_{2}>\mathrm{Pt} / \mathrm{SiO}_{2}>\mathrm{Ta}_{2} \mathrm{O}_{5} \approx \mathrm{Nb}_{2} \mathrm{O}_{5}$ and the increased activity was mainly ascribed to the formation of interfacial perimeter sites between Pt and the $\mathrm{TiO}_{2}$ or $\mathrm{CeO}_{2}$ overlayer. In addition, the overlayer made the catalyst more resistant towards sintering of Pt NP at elevated temperature.

Yang et al. ${ }^{140}$ prepared $\mathrm{Rh} / \mathrm{SiO}_{2}$ catalysts covered with $\mathrm{TiO}_{2}$ or $\mathrm{Al}_{2} \mathrm{O}_{3}$ and applied these for $\mathrm{CO}$ hydrogenation. The $\mathrm{Al}_{2} \mathrm{O}_{3}$ overlayer decreased the activity through site blockage. The $\mathrm{TiO}_{2}$ overlayer, on the other hand, increased the intrinsic activity and selectivity towards $\mathrm{C}_{5+}$ alkanes and alkenes, while the selectivity towards higher alcohols remained unchanged. Its catalytic performance was similar to that of Rh NPs on $\mathrm{TiO}_{2}$-coated $\mathrm{SiO}_{2}$, showing either that coverage of the NPs themselves was irrelevant for catalysis or that the system was dynamic and reached the same (SMSI) state during catalysis. The latter option can be rationalized considering that overlayer deposition can be used to create artificial support suboxide coverage on NPs, thereby mimicking an SMSI state. ${ }^{141}$

Treatment of $\mathrm{Au} / \mathrm{TiO}_{2}$ with $\mathrm{TiCl}_{3}$ at room temperature resulted in the formation of a $\mathrm{TiO}_{\mathrm{x}}$ overlayer on $\mathrm{Au}$, similar to SMSI. ${ }^{142}$ The $\mathrm{TiO}_{\mathrm{x}}$ overlayer increased the activity of the catalyst for CO oxidation (0.68 vs. $\left.0.26 \mathrm{~mol}_{\mathrm{CO}} \cdot \mathrm{mol}_{\mathrm{Au}}{ }^{-1} \cdot \mathrm{s}^{-1}\right)$ and brought about stability to the 
gold nanoparticles. The $\mathrm{Au}-\mathrm{TiO}_{\mathrm{x}}$ interface was identified as the active site for oxygen activation, most likely due to oxygen vacancies, which were then regenerated after reaction with $\mathrm{CO}$. The suboxides, however, also donated electrons to $\mathrm{Au}$ resulting in $\mathrm{Au}^{\delta}$ and decreasing $\mathrm{CO}$ adsorption. An oxidative pre-treatment increased the activity of the catalyst by removing the excess electrons in $\mathrm{Au}$, enhancing $\mathrm{CO}$ adsorption.

Copper phyllosilicates have been employed as precursor for the formation of silicasupported metal nanoparticles upon reduction in $\mathrm{H}_{2}$, leading to high $\mathrm{Cu}$ loadings $(\sim 40-$ $50 \mathrm{wt} . \%$ ) with uniform distribution and particle size. The role of the generated $\mathrm{Cu}-\mathrm{SiO}_{2}$ interface in these materials has been exploited, for example, in the decomposition of methanol to hydrogen and dimethoxymethane in the liquid phase, ${ }^{143}$ where $\mathrm{Cu}^{+}$and $\mathrm{Cu}-\mathrm{O}-$ Si moieties were necessary for the activity and high selectivity of the catalyst. The coppersilica interface $\left(\mathrm{Cu}-\mathrm{O}-\mathrm{SiO}_{\mathrm{x}}\right)$ was recently further optimized by use of a mesoporous silica layer coating the copper phyllosilicate prior $\mathrm{H}_{2}$ activation (Figure 6.6a). ${ }^{144}$ In ester hydrogenation, the bare $\mathrm{Cu} / \mathrm{SiO}_{2}$ obtained from copper phyllosilicate gave a TOF of $6.4 \cdot 10$ ${ }^{3} \mathrm{~S}^{-1}$ and a selectivity to ethylene glycol of $23 \%$, whereas the performance of $\mathrm{Cu} / \mathrm{SiO}_{2}$ with a mesoporous silica layer increased with a TOF of $11.4 \cdot 10^{-3} \mathrm{~s}^{-1}$ and a selectivity to ethylene glycol of $96 \%$. The $\mathrm{Cu}-\mathrm{O}-\mathrm{SiO}_{\mathrm{x}}$ interface was shown to stabilize the transition state of the ester (in this case dimethyl oxalate) and to activate $\mathrm{H}_{2}$ in a heterolytic way forming $\mathrm{Cu}-\mathrm{H}^{\delta}$ and $\mathrm{SiO}-\mathrm{H}^{\delta^{+}}$, as previously discussed for $\mathrm{H}_{2}$ activation with nanosized non-reducible supports.

\subsubsection{Adsorbate-mediated MSI}

MSI can be tuned by adsorbing specific compounds prior to inducing the MSI state. So far, this strategy has been limited to reducible supports, because the adsorbates mainly affect the coverage of the metal NPs by support suboxides. Matsubu et al. ${ }^{145}$ recently reported this strategy for the first time. They investigated $\mathrm{Rh} / \mathrm{TiO}_{2}$ catalysts for $\mathrm{CO}_{2}$ hydrogenation and found that a treatment in $20 \% \mathrm{CO}_{2}, 2 \% \mathrm{H}_{2}, 78 \%$ He atmosphere at $250{ }^{\circ} \mathrm{C}$ caused the formation of $\mathrm{HCO}_{x}$ adsorbates on the Rh NPs. In the untreated $\mathrm{Rh} / \mathrm{TiO}_{2}$ sample, a crystalline SMSI overlayer, which contained $\mathrm{Ti}^{3+}$-ions, formed during reduction at $550{ }^{\circ} \mathrm{C}$, whereas the adsorbate-mediated SMSI state, induced at only $250{ }^{\circ} \mathrm{C}$, led to an amorphous overlayer containing both $\mathrm{Ti}^{3+}$ - and $\mathrm{Ti}^{4+}$-ions (Figure 6.6b). In the adsorbate-mediated case, the support suboxides as well as the adsorbates can interact with the active sites. By covering the Rh NPs with an adsorbate-mediated SMSI overlayer, which was more stable under reaction conditions, it was possible to steer the catalyst selectivity from mainly $\mathrm{CH}_{4}$ producing (on Rh NPs) to mainly CO producing (on single Rh atoms). Rh NPs were thus more affected by the adsorbate-mediated SMSI overlayer than the single Rh atoms.

Interestingly, a similar $20 \% \mathrm{CO}_{2} / 2 \% \mathrm{H}_{2}$ pre-treatment was used by Wang et al. ${ }^{146}$ for $\mathrm{Cu} / \mathrm{CeO}_{2}$ catalysts for WGS. However, they mainly used the adsorbate-mediated SMSI strategy to prevent sintering of the $\mathrm{Cu}$ NPs and the effect on intrinsic activity was modest, although also some charge transfer from the support to the $\mathrm{Cu}$ NPs was inferred from XPS. This shows that the effect of such a treatment is highly dependent on the interplay between metal, support and reaction. 




Figure 6.6. MSI tuning strategies involving overlayer deposition and adsorbate-mediated treatments of supported metal catalysts. a Schematic representation of the effect of a silica overlayer on a copper phyllosilicate-based catalyst and the activation of dimethyl oxalate (DMO). The chart shows DMO conversion and ethylene glycol (EG) selectivity for the catalyst with and without silica overlayer (ref. ${ }^{144}$ ). b In-situ STEM observation of SMSI in $\mathrm{Rh} / \mathrm{TiO}_{2}$ after a treatment in $\mathrm{H}_{2}$ at $550{ }^{\circ} \mathrm{C}$ which induced a $\mathrm{TiO}_{\mathrm{x}}$ bilayer (SMSI overlayer) and after treatment in $20 \mathrm{CO}_{2}: 2 \mathrm{H}_{2}$ at $250{ }^{\circ} \mathrm{C}$ which induced an amorphous $\mathrm{TiO}_{\mathrm{x}}$ overlayer (A-SMSI overlayer). Production rates of $\mathrm{CH}_{4}$ (red) and $\mathrm{CO}$ (grey) are shown on the chart for Rh supported on alumina or titania after $\mathrm{H}_{2}$ or $20 \mathrm{CO}_{2}: 2 \mathrm{H}_{2}$ treatments (ref. $\left.{ }^{145}\right)$.

\subsection{Discussion}

We analyzed the literature to quantify the enhancement of catalytic performance that was achieved in recent years through MSI modifications. We limited the analysis largely to $\mathrm{C}_{1}$ chemistry, i.e., catalytic reactions involving $\mathrm{CO}, \mathrm{CH}_{4}, \mathrm{CO}_{2}$ or methanol as the main reactant. The performance of tuned catalysts was benchmarked against a reference catalyst reported in the same paper and therefore investigated under the same conditions. Consequently, an enhancement factor for the tuned catalyst over the reference one was obtained. This approach provides a measure for the efficiency of the various MSI tuning strategies and facilitates comparison between different papers reporting a wide variety of reactions, conditions and units.

The rate of formation (productivity) of desirable products, i.e., the product of total activity and selectivity, was taken as a measure for catalytic performance. Productivity was used because this shows the combined effect of activity and selectivity changes and hence 
provides a more complete picture of the effect of MSI tuning than either parameter alone. Turnover frequencies or specific activities were preferred over conversions as activity descriptors, because these provide more information about intrinsic catalytic activities. Furthermore, selectivities are not relevant for certain reactions such as CO oxidation reactions. For these systems, $100 \%$ selectivity is assumed and productivity enhancement is essentially determined by activity differences.

Figure 6.7a shows the productivity enhancement as a function of the different MSI tuning methods. Relative productivity enhancements up to a factor of $\sim 15$ have been obtained using various tuning methods, irrespective of which catalyst component is altered. Support modifications were effective in altering all MSI phenomena with substantial increase of the corresponding productivity. Particularly, selection of a certain support composition ${ }^{113}$ or adding dopants to the support ${ }^{85}$ strongly improved the performance due to changes in the overall catalyst chemical composition. Treatments on the as-synthetized catalysts to tune the MSI have also proven beneficial to enhance their performance, most effort has focused on modifying the SMSI effect from which induction of this phenomenon on $\mathrm{Au}$ nanoparticles by thermal treatment ${ }^{120}$ and use of overlayer deposition of transition metal oxides ${ }^{139,140}$ led to significant catalytic enhancement. Reports involving strategies based on NP modifications to affect MSI were fewer, however an approach leading to substantially increased performance involved changing NP size which in turn improved the interfacial contribution for $\mathrm{CO}_{2}$ hydrogenation of a $\mathrm{Ru} / \mathrm{CeO}_{2}$ catalyst. ${ }^{107}$

For the enhancement of selectivity, the largest increases were reported for treatments to modify MSI in catalysts for $\mathrm{CO}_{2}$ hydrogenation (78-85\%), where preferential blockage/sintering of small Rh or Ru NPs or single atoms leads to a strong shift in selectivity from $\mathrm{CH}_{4}$ towards $\mathrm{CO} .{ }^{145,147}$ However, these strong increases in selectivity were accompanied by decreased activity, leading to productivity enhancement factor between 3 and 6 . Besides these two examples, selectivity increases of $20-30 \%$ are occasionally reported for various reactions. Furthermore, $\mathrm{CO}$ oxidation and reduction reactions were investigated most; 22 studies focused on CO oxidation and 11 on CO reduction reactions out of 50 papers analyzed.

The enhancement of productivity can be correlated with metal particle size of the catalyst after MSI tuning (Figure 6.7b, dataset divided on the main MSI phenomenon that was affected). In general, the efficiency of MSI tuning rapidly decayed with increasing particle size and the highest enhancements were obtained for metal particles smaller than $4 \mathrm{~nm}$ through modification of any MSI phenomenon. The majority of studies focused on catalysts with particle sizes in the $<4 \mathrm{~nm}$ size range. For metal particles that are larger than $4 \mathrm{~nm}$, the gain in productivity as a result of MSI tuning is modest in most cases, although a few exceptions were reported. Furthermore, for these larger particles, SMSI was most often the dominant phenomenon, implying that SMSI is less affected by metal nanoparticle size. This can be rationalized by the support covering the NP and thus being in contact with a large fraction of the metal surface, regardless of particle size in the range described here. 

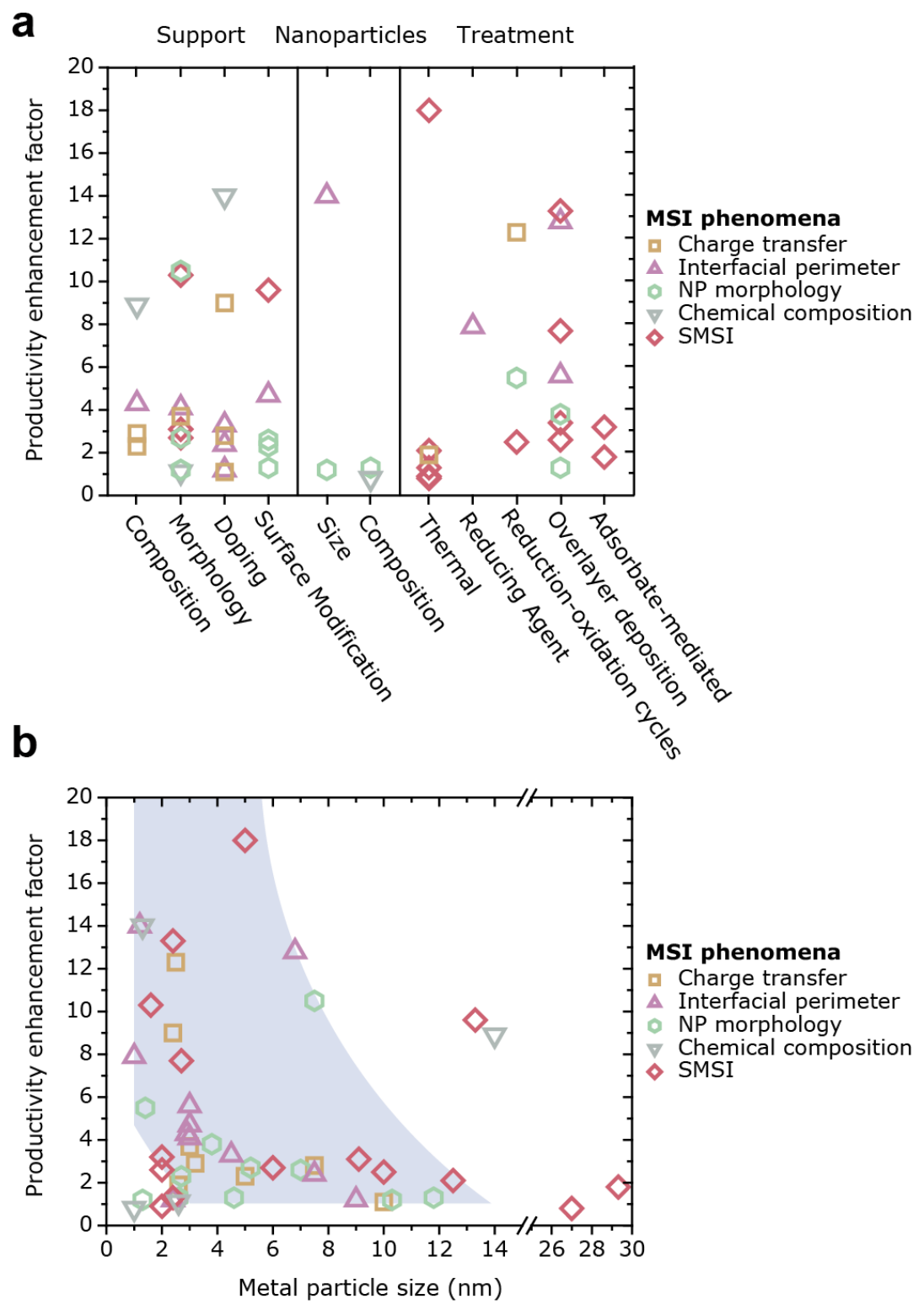

Figure 6.7. Enhancement of catalytic performance in $C_{1}$ chemistry achieved through control of MSI in recent years. a, Relative differences in productivity of a desired compound for the different MSI tuning strategies grouped by general strategy (support, nanoparticle and treatment) and with different symbols indicating the main MSI phenomenon affected. Tuning strategies by modifications on the support or post-synthetic treatments have been mostly explored. b, Productivity enhancement factor as a function of metal particle size of the optimal catalyst, separated on the main MSI phenomenon affected by tuning. The grey-blue area is added as a guide to the eye and indicates the domain in which the NP size brought about the strongest productivity enhancement.

When considering many types of catalysis, it became apparent that mainly late transition metals, starting from group 10, were involved when charge transfer between metal and support played a role in the catalysis. As for the supports, charge transfer with the metal required either conductive supports (e.g., graphite), or semi conductive supports (reducible metal oxides), often modified by dopants to increase conductivity further. 
Insulators (irreducible oxides) require dopants or functionalization for charge transfer to occur on a noticeable scale..$^{101}$ This can be explained by a mismatch in band positions between insulating supports and the nanoparticles, i.e., the valence band of the insulator being too low in energy to affect the electronic properties of the metal NP.

Another observation is that the work on MSI modifications for electro- and photocatalysis is limited. On one hand, this might be ascribed to early development of the role MSI in these fields of catalysis and on the other hand, the range of applicable supports/metals might be more limited. ${ }^{148}$ Nevertheless, we believe that the development of MSI tuning methods could be worthwhile for these applications.

Overall, MSI can improve catalytic performance roughly by one order of magnitude under industrially relevant conditions. This effect may seem modest compared to, for example, a difference of four orders of magnitude in $\mathrm{CO}$ hydrogenation activity that is obtained with various metals from groups $8-10$ in the periodic table. ${ }^{149}$ However, often the selection of the metal is fixed by selectivity considerations and MSI modification is one of the few effective tools for designing and enhancing catalyst performance. In addition, these results show that supports are more than merely an inert structural matrix and that their chemical properties can be applied to substantially improve catalytic performance.

\section{Acknowledgements}

Shell Global Solutions, the Netherlands Association for Scientific Research (NWO) and Companhia Brasileira de Metalurgia e Mineração (CBMM) are thanked for financial support. K.P.d.J. acknowledges support from the European Research Council, EU FP7 ERC Advanced Grant no. 338846.

\section{References}

1. Anderson, J. A. \& García, M. F. Supported Metals in Catalysis. Catalytic Science Series Volume 5, (Imperial College Press, 2005).

2. Roldan Cuenya, B. Synthesis and catalytic properties of metal nanoparticles: Size, shape, support, composition, and oxidation state effects. Thin Solid Films 518, 3127-3150 (2010).

3. Zečević, J., Vanbutsele, G., de Jong, K. P. \& Martens, J. A. Nanoscale intimacy in bifunctional catalysts for selective conversion of hydrocarbons. Nature 528, 245-248 (2015).

4. Arnal, P. M., Comotti, M. \& Schüth, F. High-Temperature-Stable Catalysts by Hollow Sphere Encapsulation. Angew. Chem. Int. Ed. 45, 8224-8227 (2006).

5. Pacchioni, G. \& Freund, H.-J. Controlling the charge state of supported nanoparticles in catalysis: lessons from model systems. Chem. Soc. Rev. 47, 8474-8502 (2018).

6. Farmer, J. a \& Campbell, C. T. Ceria Maintains Smaller Metal Catalyst Particles by Strong MetalSupport Bonding. Science 329, 933-936 (2010).

7. Ahmadi, M., Mistry, H. \& Roldan Cuenya, B. Tailoring the Catalytic Properties of Metal Nanoparticles via Support Interactions. J. Phys. Chem. Lett. 7, 3519-3533 (2016).

8. Ro, I., Resasco, J. \& Christopher, P. Approaches for Understanding and Controlling Interfacial Effects in Oxide-Supported Metal Catalysts. ACS Catal. 8, 7368-7387 (2018). 
9. Pan, C.-J. et al. Tuning/exploiting Strong Metal-Support Interaction (SMSI) in Heterogeneous Catalysis. J. Taiwan Inst. Chem. Eng. 74, 154-186 (2017).

10. Fujiwara, K., Okuyama, K. \& Pratsinis, S. E. Metal-support interactions in catalysts for environmental remediation. Environ. Sci. Nano 4, 2076-2092 (2017).

11. Chen, M. S. \& Goodman, D. W. The Structure of Catalytically Active Gold on Titania. Science 306, 252-255 (2004).

12. Luches, P. et al. Nature of Ag Islands and Nanoparticles on the $\mathrm{CeO}_{2}(111)$ Surface. J. Phys. Chem. C116, 1122-1132 (2011).

13. Pacchioni, G. Electronic interactions and charge transfers of metal atoms and clusters on oxide surfaces. Phys. Chem. Chem. Phys. 15, 1737-1757 (2013).

14. Puigdollers, A. R., Schlexer, P., Tosoni, S. \& Pacchioni, G. Increasing Oxide Reducibility: The Role of Metal/Oxide Interfaces in the Formation of Oxygen Vacancies. ACS Catal. 7, 6493-6513 (2017).

15. Molina, L. M. \& Hammer, B. Some recent theoretical advances in the understanding of the catalytic activity of Au. Appl. Catal. A Gen. 291, 21-31 (2005).

16. Zhang, B. \& Qin, Y. Interface Tailoring of Heterogeneous Catalysts by Atomic Layer Deposition. ACS Catal. 8, 10064-10081 (2018).

17. Lin, $\mathrm{X}$. et al. Characterizing low-coordinated atoms at the periphery of $\mathrm{MgO}$-supported $\mathrm{Au}$ islands using scanning tunneling microscopy and electronic structure calculations. Phys. Rev. B - Condens. Matter Mater. Phys. 81, 6-9 (2010).

18. Hammer, B. Special Sites at Noble and Late Transition Metal Catalysts. Top. Catal. 37, 3-16 (2006).

19. Farnesi Camellone, M., Negreiros Ribeiro, F., Szabová, L., Tateyama, Y. \& Fabris, S. Catalytic Proton Dynamics at the Water/Solid Interface of Ceria-Supported Pt Clusters. J. Am. Chem. Soc. 138, 11560-11567 (2016).

20. Toebes, M. L. et al. Support effects in hydrogenation of cinnamaldehyde over carbon nanofibersupported platinum catalysts: Kinetic modeling. Chem. Eng. Sci. 60, 5682-5695 (2005).

21. Davis, S. E., Ide, M. S. \& Davis, R. J. Selective oxidation of alcohols and aldehydes over supported metal nanoparticles. Green Chem. 15, 17-45 (2013).

22. Conner, W. C. \& Falconer, J. L. Spillover in Heterogeneous Catalysis. Chem. Rev. 95, 759-788 (1995).

23. Prins, R. Hydrogen Spillover. Facts and Fiction. Chem. Rev. 112, 2714-2738 (2012).

24. Takakusagi, S., Fukui, K. I., Tero, R., Asakura, K. \& Iwasawa, Y. First Direct Visualization of Spillover Species Emitted from Pt Nanoparticles. Langmuir 26, 16392-16396 (2010).

25. Karim, W. et al. Catalyst support effects on hydrogen spillover. Nature 541, 68-71 (2017).

26. Roldan Cuenya, B. Metal Nanoparticle Catalysts Beginning to Shape-up. Acc. Chem. Res. 46, 1682-1691 (2012).

27. Frenkel, A. I. et al. Correlating Particle Size and Shape of Supported $\mathrm{Ru} / \mathrm{\gamma}-\mathrm{Al}_{2} \mathrm{O}_{3}$ Catalysts with $\mathrm{NH}_{3}$ Decomposition Activity. J. Am. Chem. Soc. 131, 12230-12239 (2009).

28. Henry, C. R. Morphology of supported nanoparticles. Prog. Surf. Sci. 80, 92-116 (2005).

29. Hansen, P. L. et al. Atom-Resolved Imaging of Dynamic Shape Changes in Supported Copper Nanocrystals. Science 295, 2053-2055 (2002). 
30. Hemmingson, S. L. \& Campbell, C. T. Trends in Adhesion Energies of Metal Nanoparticles on Oxide Surfaces: Understanding Support Effects in Catalysis and Nanotechnology. ACS Nano 11, 1196-1203 (2017).

31. Ahmadi, M., Behafarid, F. \& Roldan Cuenya, B. Size-dependent adhesion energy of shapeselected Pd and Pt nanoparticles. Nanoscale 8, 11635-11641 (2016).

32. Tanase, M. et al. Interfacial Bonding Stabilizes Rhodium and Rhodium Oxide Nanoparticles on Layered Nb Oxide and Ta Oxide Supports. J. Am. Chem. Soc. 136, 5687-5696 (2014).

33. Duan, M. et al. Reconstruction of Supported Metal Nanoparticles in Reaction Conditions. Angew. Chem. Int. Ed. 57, 6464-6469 (2018).

34. Lin, Y. et al. Adhesion and Atomic Structures of Gold on Ceria Nanostructures: The Role of Surface Structure and Oxidation State of Ceria Supports. Nano Lett. 15, 5375-5381 (2015).

35. Pingel, T. N., Jørgensen, M., Yankovich, A. B., Grönbeck, H. \& Olsson, E. Influence of atomic site-specific strain on catalytic activity of supported nanoparticles. Nat. Commun. 9, 2722 (2018).

36. Shibata, N. et al. Interface Structures of Gold Nanoparticles on $\mathrm{TiO}_{2}(110)$. Phys. Rev. Lett. 102, 136105 (2009).

37. Bartholomew, C. H. Mechanisms of catalyst deactivation. Appl. Catal. A Gen. 212, 17-60 (2001).

38. van Deelen, T. W., Nijhuis, J. J., Krans, N. A., Zec ``ević, J. \& de Jong, K. P. Preparation of Cobalt Nanocrystals Supported on Metal Oxides To Study Particle Growth in Fischer-Tropsch Catalysts. ACS Catal. 8, 10581-10589 (2018).

39. Penner, S. \& Armbrüster, M. Formation of Intermetallic Compounds by Reactive MetalSupport Interaction: A Frequently Encountered Phenomenon in Catalysis. ChemCatChem 7, 374-392 (2015).

40. Furukawa, S. \& Komatsu, T. Intermetallic Compounds: Promising Inorganic Materials for WellStructured and Electronically Modified Reaction Environments for Efficient Catalysis. ACS Catal. 7, 735-765 (2017).

41. Zafeiratos, S., Piccinin, S. \& Teschner, D. Alloys in catalysis: phase separation and surface segregation phenomena in response to the reactive environment. Catal. Sci. Technol. 2, 17871801 (2012).

42. Singh, A. K. \& Xu, Q. Synergistic Catalysis over Bimetallic Alloy Nanoparticles. ChemCatChem 5, 652-676 (2013).

43. Ferrando, R., Jellinek, J. \& Johnston, R. L. Nanoalloys: From Theory to Applications of Alloy Clusters and Nanoparticles. Chem. Rev. 108, 845-910 (2008).

44. Tauster, S. J., Fung, S. C. \& Garten, R. L. Strong metal-support interactions. Group 8 noble metals supported on titanium dioxide. J. Am. Chem. Soc. 100, 170-175 (1978).

45. Tauster, S. J., Fung, S. C., Baker, R. T. \& Horsley, J. A. Strong Interactions in Supported-Metal Catalysts. Science 211, 1121-1125 (1981).

46. Hernández-Cristobal, O., Arenas-Alatorre, J., Díaz, G., Bahena, D. \& J. Yacamán, M. High Resolution HAADF Characterization of $\mathrm{Ir} / \mathrm{TiO}_{2}$ Catalyst Reduced at $500{ }^{\circ} \mathrm{C}$ : Intensity Profile Analysis. J. Phys. Chem. C119, 11672-11678 (2015).

47. Willinger, M. G. et al. A Case of Strong Metal-Support Interactions: Combining Advanced Microscopy and Model Systems to Elucidate the Atomic Structure of Interfaces. Angew. Chem. Int. Ed. 53, 5998-6001 (2014). 
48. Zhang, S. et al. Dynamical Observation and Detailed Description of Catalysts under Strong Metal-Support Interaction. Nano Lett. 16, 4528-4534 (2016).

49. Chen, M. S. \& Goodman, D. W. Interaction of Au with titania: the role of reduced Ti. Top. Catal. 44, 41-47 (2007).

50. Saavedra, J., Pursell, C. J. \& Chandler, B. D. CO Oxidation Kinetics over $\mathrm{Au} / \mathrm{TiO}_{2}$ and $\mathrm{Au} / \mathrm{Al}_{2} \mathrm{O}_{3}$ Catalysts: Evidence for a Common Water-Assisted Mechanism. J. Am. Chem. Soc. 140, 37123723 (2018).

51. Wang, Y., Widmann, D. \& Behm, R. J. Influence of $\mathrm{TiO}_{2}$ Bulk Defects on CO Adsorption and CO Oxidation on $\mathrm{Au} / \mathrm{TiO}_{2}$ : Electronic Metal-Support Interactions (EMSIs) in Supported $\mathrm{Au}$ Catalysts. ACS Catal. 7, 2339-2345 (2017).

52. Wang, Y. et al. The role of electronic metal-support interactions and its temperature dependence: $\mathrm{CO}$ adsorption and $\mathrm{CO}$ oxidation on $\mathrm{Au} / \mathrm{TiO}_{2}$ catalysts in the presence of $\mathrm{TiO}_{2}$ bulk defects. J. Catal. 354, 46-60 (2017).

53. Kumar, G. et al. Evaluating differences in the active-site electronics of supported Au nanoparticle catalysts using Hammett and DFT studies. Nat. Chem. 10, 268-274 (2018).

54. Song, H. et al. Visible-Light-Mediated Methane Activation for Steam Methane Reforming under Mild Conditions: A Case Study of Rh/ $\mathrm{TiO}_{2}$ Catalysts. ACS Catal. 8, 7556-7565 (2018).

55. Sakamoto, H. et al. Hot-Electron-Induced Highly Efficient $\mathrm{O}_{2}$ Activation by Pt Nanoparticles Supported on $\mathrm{Ta}_{2} \mathrm{O}_{5}$ Driven by Visible Light. J. Am. Chem. Soc. 137, 9324-9332 (2015).

56. Jackson, C. et al. Electronic metal-support interaction enhanced oxygen reduction activity and stability of boron carbide supported platinum. Nat. Commun. 8, 15802 (2017).

57. Irvine, J. T. S. et al. Evolution of the electrochemical interface in high-temperature fuel cells and electrolysers. Nat. Energy 1, 1-13 (2016).

58. Neagu, D., Tsekouras, G., Miller, D. N., Ménard, H. \& Irvine, J. T. S. In situ growth of nanoparticles through control of non-stoichiometry. Nat. Chem. 5, 916-923 (2013).

59. Neagu, D. et al. Nano-socketed nickel particles with enhanced coking resistance grown in situ by redox exsolution. Nat. Commun. 6, 8120 (2015).

60. Gao, Y., Wang, J., Lyu, Y.-Q., Lam, K. \& Ciucci, F. In situ growth of $\mathrm{Pt}_{3} \mathrm{Ni}$ nanoparticles on an A-site deficient perovskite with enhanced activity for the oxygen reduction reaction. J. Mater. Chem. A 5, 6399-6404 (2017).

61. Huang, X., Zhao, G., Wang, G. \& Irvine, J. T. S. Synthesis and applications of nanoporous perovskite metal oxides. Chem. Sci. 9, 3623-3637 (2018).

62. Murata, K. et al. The Metal-Support Interaction Concerning the Particle Size Effect of $\mathrm{Pd} / \mathrm{Al}_{2} \mathrm{O}_{3}$ on Methane Combustion. Angew. Chem. Int. Ed. 56, 15993-15997 (2017).

63. Bertella, F., Concepción, P. \& Martínez, A. $\mathrm{TiO}_{2}$ polymorph dependent SMSI effect in Co$\mathrm{Ru} / \mathrm{TiO}_{2}$ catalysts and its relevance to Fischer-Tropsch synthesis. Catal. Today 289, 181-191 (2017).

64. Yu, L. et al. Influence of the Crystal Structure of Titanium Oxide on the Catalytic Activity of $\mathrm{Rh} / \mathrm{TiO}_{2}$ in Steam Reforming of Propane at Low Temperature. Chem. - Eur. J. 24, 8742-8746 (2018).

65. Bertella, F., Concepción, P. \& Martínez, A. The impact of support surface area on the SMSI decoration effect and catalytic performance for Fischer-Tropsch synthesis of $\mathrm{Co}-\mathrm{Ru} / \mathrm{TiO}_{2}-$ anatase catalysts. Catal. Today 296, 170-180 (2017). 
66. Abdel-Mageed, A. M. et al. Selective $\mathrm{CO}$ Methanation on $\mathrm{Ru} / \mathrm{TiO}_{2}$ Catalysts: Role and Influence of Metal-Support Interactions. ACS Catal. 5, 6753-6763 (2015).

67. Yoon, S. et al. Specific Metal-Support Interactions between Nanoparticle Layers for Catalysts with Enhanced Methanol Oxidation Activity. ACS Catal. 8, 5391-5398 (2018).

68. Lin, B. et al. Effect of ceria morphology on the catalytic activity of $\mathrm{Co} / \mathrm{CeO}_{2}$ catalyst for ammonia synthesis. Catal. Commun. 101, 15-19 (2017).

69. Ma, Z., Zhao, S., Pei, X., Xiong, X. \& Hu, B. New insights into the support morphologydependent ammonia synthesis activity of $\mathrm{Ru} / \mathrm{CeO}_{2}$ catalysts. Catal. Sci. Technol. 7, 191-199 (2017).

70. Ha, H., Yoon, S., An, K. \& Kim, H. Y. Catalytic CO Oxidation over Au Nanoparticles Supported on $\mathrm{CeO}_{2}$ Nanocrystals: Effect of the $\mathrm{Au}-\mathrm{CeO}_{2}$ Interface. ACS Catal. 8, 11491-11501 (2018).

71. Liu, M.-H., Chen, Y.-W., Lin, T.-S. \& Mou, C.-Y. Defective Mesocrystal ZnO-Supported Gold Catalysts: Facilitating CO Oxidation via Vacancy Defects in ZnO. ACS Catal. 8, 6862-6869 (2018).

72. Zhu, W. et al. Taming interfacial electronic properties of platinum nanoparticles on vacancyabundant boron nitride nanosheets for enhanced catalysis. Nat. Commun. 8, 15291 (2017).

73. Yan, X. et al. Nickel@Siloxene catalytic nanosheets for high-performance $\mathrm{CO}_{2}$ methanation. Nat. Commun. 10, 2608 (2019).

74. Zhang, F. et al. Tailoring the Oxidation Activity of Pt Nanoclusters via Encapsulation. ACS Catal. 5, 1381-1385 (2015).

75. Li, Z. et al. Reactive metal-support interactions at moderate temperature in two-dimensional niobium-carbide-supported platinum catalysts. Nat. Catal. 1, 349-355 (2018).

76. Li, Z. et al. Two-dimensional transition metal carbides as supports for tuning the chemistry of catalytic nanoparticles. Nat. Commun. 9, 5258 (2018).

77. Shi, L., Li, Z., Dao, T. D., Nagao, T. \& Yang, Y. A synergistic interaction between isolated Au nanoparticles and oxygen vacancies in an amorphous black $\mathrm{TiO}_{2}$ nanoporous film: Toward enhanced photoelectrochemical water splitting. J. Mater. Chem. A 6, 12978-12984 (2018).

78. He, L., Weniger, F., Neumann, H. \& Beller, M. Synthesis, Characterization, and Application of Metal Nanoparticles Supported on Nitrogen-Doped Carbon: Catalysis beyond Electrochemistry. Angew. Chem. Int. Ed. 55, 12582-12594 (2016).

79. Shi, R. et al. Nitrogen-doped graphene supported copper catalysts for methanol oxidative carbonylation: Enhancement of catalytic activity and stability by nitrogen species. Carbon 130, 185-195 (2018).

80. Ning, X. et al. Electron transfer dependent catalysis of Pt on N-doped carbon nanotubes: Effects of synthesis method on metal-support interaction. J. Catal. 348, 100-109 (2017).

81. Walczak, R. et al. Template- and Metal-Free Synthesis of Nitrogen-Rich Nanoporous "Noble" Carbon Materials by Direct Pyrolysis of a Preorganized Hexaazatriphenylene Precursor. Angew. Chem. Int. Ed. 57, 10765-10770 (2018).

82. Antonietti, M. \& Oschatz, M. The Concept of "Noble, Heteroatom-Doped Carbons," Their Directed Synthesis by Electronic Band Control of Carbonization, and Applications in Catalysis and Energy Materials. Adv. Mater. 30, 1706836 (2018). 
83. Qin, Q., Heil, T., Antonietti, M. \& Oschatz, M. Single-Site Gold Catalysts on Hierarchical NDoped Porous Noble Carbon for Enhanced Electrochemical Reduction of Nitrogen. Small Methods 2, 1800202 (2018).

84. Theofanidis, S. A. et al. Fe-Containing Magnesium Aluminate Support for Stability and Carbon Control during Methane Reforming. ACS Catal. 8, 5983-5995 (2018).

85. Margossian, T. et al. Molecularly Tailored Nickel Precursor and Support Yield a Stable Methane Dry Reforming Catalyst with Superior Metal Utilization. J. Am. Chem. Soc. 139, 6919-6927 (2017).

86. Horlyck, J., Lewis, S., Amal, R. \& Scott, J. The Impact of La Doping on Dry Reforming Ni-Based Catalysts Loaded on FSP-Alumina. Top. Catal. 61, 1842-1855 (2018).

87. Hsieh, B.-J. et al. Platinum loaded on dual-doped $\mathrm{TiO}_{2}$ as an active and durable oxygen reduction reaction catalyst. NPG Asia Mater. 9, e403 (2017).

88. Tran, S. B. T., Choi, H. S., Oh, S. Y., Moon, S. Y. \& Park, J. Y. Iron-doped ZnO as a support for Pt-based catalysts to improve activity and stability: Enhancement of metal-support interaction by the doping effect. RSC Adv. 8, 21528-21533 (2018).

89. Wang, F. et al. Enhanced catalytic performance of Ir catalysts supported on ceria-based solid solutions for methane dry reforming reaction. Catal. Today 281, 295-303 (2017).

90. Tabakova, T. et al. Structure-activity relationship in water-gas shift reaction over gold catalysts supported on Y-doped ceria. J. Rare Earths 37, 383-392 (2019).

91. Chen, P. et al. Experimental and Theoretical Understanding of Nitrogen-Doping-Induced Strong Metal-Support Interactions in $\mathrm{Pd} / \mathrm{TiO}_{2}$ Catalysts for Nitrobenzene Hydrogenation. ACS Catal. 7, 1197-1206 (2017).

92. Schumann, J. et al. Promoting Strong Metal Support Interaction: Doping ZnO for Enhanced Activity of Cu/ZnO:M (M = Al, Ga, Mg) Catalysts. ACS Catal. 5, 3260-3270 (2015).

93. Chernyak, S. A. et al. Effect of Co crystallinity on $\mathrm{Co} / \mathrm{CNT}$ catalytic activity in $\mathrm{CO} / \mathrm{CO}_{2}$ hydrogenation and CO disproportionation. Appl. Surf. Sci. 372, 100-107 (2016).

94. Chernyak, S. A. et al. Co catalysts supported on oxidized CNTs: evolution of structure during preparation, reduction and catalytic test in Fischer-Tropsch synthesis. Appl. Catal. A Gen. 523, 221-229 (2016).

95. Eschemann, T. O. et al. Effect of support surface treatment on the synthesis, structure, and performance of Co/CNT Fischer-Tropsch catalysts. J. Catal. 328, 130-138 (2015).

96. Honma, T. \& Wayman, C. M. Epitaxial Growth of Evaporated Cobalt Films. J. Appl. Phys. 36, 2791-2798 (1965).

97. Rao, R. G. et al. Interfacial charge distributions in carbon-supported palladium catalysts. Nat. Commun. 8, 340 (2017).

98. Shi, W. et al. Enhanced Chemoselective Hydrogenation through Tuning the Interaction between Pt Nanoparticles and Carbon Supports: Insights from Identical Location Transmission Electron Microscopy and X-ray Photoelectron Spectroscopy. ACS Catal. 6, 7844-7854 (2016).

99. Donoeva, B., Masoud, N. \& De Jongh, P. E. Carbon Support Surface Effects in the GoldCatalyzed Oxidation of 5-Hydroxymethylfurfural. ACS Catal. 7, 4581-4591 (2017).

100. Celebi, M., Yurderi, M., Bulut, A., Kaya, M. \& Zahmakiran, M. Palladium nanoparticles supported on amine-functionalized $\mathrm{SiO}_{2}$ for the catalytic hexavalent chromium reduction. Appl. Catal. B Environ. 180, 53-64 (2016). 
101. Rodríguez-Gómez, A., Platero, F., Caballero, A. \& Colón, G. Improving the direct synthesis of hydrogen peroxide from hydrogen and oxygen over $\mathrm{Au}-\mathrm{Pd} / \mathrm{SBA}-15$ catalysts by selective functionalization. Mol. Catal. 445, 142-151 (2018).

102. Van Den Berg, R. et al. Support Functionalization To Retard Ostwald Ripening in Copper Methanol Synthesis Catalysts. ACS Catal. 5, 4439-4448 (2015).

103. Pan, Y.-X. et al. Photocatalytic $\mathrm{CO}_{2}$ Reduction by Carbon-Coated Indium-Oxide Nanobelts. $J$. Am. Chem. Soc. 139, 4123-4129 (2017).

104. Prieto, G. et al. Cobalt-Catalyzed Fischer-Tropsch Synthesis: Chemical Nature of the Oxide Support as a Performance Descriptor. ACS Catal. 5, 3323-3335 (2015).

105. Liu, L. \& Corma, A. Metal Catalysts for Heterogeneous Catalysis: From Single Atoms to Nanoclusters and Nanoparticles. Chem. Rev. 118, 4981-5079 (2018).

106. Lykhach, Y. et al. Counting electrons on supported nanoparticles. Nat. Mater. 15, 284-288 (2016).

107. Guo, Y. et al. Low-Temperature $\mathrm{CO}_{2}$ Methanation over $\mathrm{CeO}_{2}$-Supported $\mathrm{Ru}$ Single Atoms, Nanoclusters, and Nanoparticles Competitively Tuned by Strong Metal-Support Interactions and H-Spillover Effect. ACS Catal. 8, 6203-6215 (2018).

108. Yan, $\mathrm{Y}$. et al. $\mathrm{Ru} / \mathrm{Al}_{2} \mathrm{O}_{3}$ catalyzed $\mathrm{CO}_{2}$ hydrogenation: Oxygen-exchange on metal-support interfaces. J. Catal. 367, 194-205 (2018).

109. Demiroglu, I. et al. Modelling free and oxide-supported nanoalloy catalysts: comparison of bulk-immiscible $\mathrm{Pd}-\mathrm{Ir}$ and $\mathrm{Au}-\mathrm{Rh}$ systems and influence of a $\mathrm{TiO}_{2}$ support. Faraday Discuss. 208, 53-66 (2018).

110. Piccolo, L. et al. Understanding and controlling the structure and segregation behaviour of AuRh nanocatalysts. Sci. Rep. 6, 1-8 (2016).

111. Konuspayeva, Z. et al. Au-Rh and Au-Pd nanocatalysts supported on rutile titania nanorods: Structure and chemical stability. Phys. Chem. Chem. Phys. 17, 28112-28120 (2015).

112. Han, C. W. et al. Highly Stable Bimetallic AuIr $/ \mathrm{TiO}_{2}$ Catalyst: Physical Origins of the Intrinsic High Stability against Sintering. Nano Lett. 15, 8141-8147 (2015).

113. Destro, P. et al. The Crucial Role of the Support in the Transformations of Bimetallic Nanoparticles and Catalytic Performance. ACS Catal. 8, 1031-1037 (2018).

114. Seemala, B., Cai, C. M., Wyman, C. E. \& Christopher, P. Support Induced Control of Surface Composition in $\mathrm{Cu}-\mathrm{Ni} / \mathrm{TiO}_{2}$ Catalysts Enables High Yield Co-Conversion of HMF and Furfural to Methylated Furans. ACS Catal. 7, 4070-4082 (2017).

115. Liu, D. et al. Identifying Dynamic Structural Changes of Active Sites in Pt-Ni Bimetallic Catalysts Using Multimodal Approaches. ACS Catal. 8, 4120-4131 (2018).

116. Divins, N. J., Angurell, I., Escudero, C., Pérez-Dieste, V. \& Llorca, J. Influence of the support on surface rearrangements of bimetallic nanoparticles in real catalysts. Science 346, 620-623 (2014).

117. Gubó, R. et al. Variation of SMSI with the Au:Pd Ratio of Bimetallic Nanoparticles on $\mathrm{TiO}_{2}(110)$. Top. Catal. 61, 308-317 (2018).

118. Zhan, W. et al. Surfactant-Assisted Stabilization of Au Colloids on Solids for Heterogeneous Catalysis. Angew. Chem. Int. Ed. 56, 4494-4498 (2017).

119. Gao, X., Liu, H., Hidajat, K. \& Kawi, S. Anti-Coking Ni/SiO ${ }_{2}$ Catalyst for Dry Reforming of Methane: Role of Oleylamine/Oleic Acid Organic Pair. ChemCatChem 7, 4188-4196 (2015). 
120. Tang, H. et al. Classical strong metal-support interactions between gold nanoparticles and titanium dioxide. Sci. Adv. 3, e1700231 (2017).

121. Tang, H. et al. Strong Metal-Support Interactions between Gold Nanoparticles and Nonoxides. J. Am. Chem. Soc. 138, 56-59 (2016).

122. Tang, H. et al. Oxidative strong metal-support interactions (OMSI) of supported platinumgroup metal catalysts. Chem. Sci. 9, 6679-6684 (2018).

123. Wang, L. et al. Strong Metal-Support Interactions Achieved by Hydroxide-to-Oxide Support Transformation for Preparation of Sinter-Resistant Gold Nanoparticle Catalysts. ACS Catal. 7, 7461-7465 (2017).

124. Hernández Mejía, C., Vogt, C., Weckhuysen, B. M. \& de Jong, K. P. Stable niobia-supported nickel catalysts for the hydrogenation of carbon monoxide to hydrocarbons. Catal. Today https://doi.org/10.1016/j.cattod.2018.11.036 (2018).

125. $\mathrm{Xu}, \mathrm{M}$. et al. $\mathrm{TiO}_{2-\mathrm{x}}-$ Modified Ni Nanocatalyst with Tunable Metal-Support Interaction for Water-Gas Shift Reaction. ACS Catal. 7, 7600-7609 (2017).

126. Li, Y. et al. High temperature reduction dramatically promotes $\mathrm{Pd} / \mathrm{TiO}_{2}$ catalyst for ambient formaldehyde oxidation. Appl. Catal. B Environ. 217, 560-569 (2017).

127. Ryabchuk, P. et al. Intermetallic nickel silicide nanocatalyst-A non-noble metal-based general hydrogenation catalyst. Sci. Adv. 4, eaat0761 (2018).

128. Serna, P. \& Corma, A. Transforming Nano Metal Nonselective Particulates into Chemoselective Catalysts for Hydrogenation of Substituted Nitrobenzenes. ACS Catal. 5, 7114-7121 (2015).

129. Rui, Z., Chen, L., Chen, H. \& Ji, H. Strong Metal-Support Interaction in Pt/ $/ \mathrm{TiO}_{2}$ Induced by Mild $\mathrm{HCHO}$ and $\mathrm{NaBH}_{4}$ Solution Reduction and Its Effect on Catalytic Toluene Combustion. Ind. Eng. Chem. Res. 53, 15879-15888 (2014).

130. Gänzler, A. M. et al. Tuning the Structure of Platinum Particles on Ceria In Situ for Enhancing the Catalytic Performance of Exhaust Gas Catalysts. Angew. Chem. Int. Ed. 56, 13078-13082 (2017).

131. Gänzler, A. M. et al. Tuning the $\mathrm{Pt} / \mathrm{CeO}_{2}$ Interface by in Situ Variation of the Pt Particle Size. ACS Catal. 8, 4800-4811 (2018).

132. Freakley, S. J. et al. Palladium-tin catalysts for the direct synthesis of $\mathrm{H}_{2} \mathrm{O}_{2}$ with high selectivity. Science 351, 965-968 (2016).

133. Hernández Mejía, C., van Deelen, T. W. \& de Jong, K. P. Activity enhancement of cobalt catalysts by tuning metal-support interactions. Nat. Commun. 9, 4459 (2018).

134. Zhan, W. et al. A Sacrificial Coating Strategy Toward Enhancement of Metal-Support Interaction for Ultrastable Au Nanocatalysts. J. Am. Chem. Soc. 138, 16130-16139 (2016).

135. Phaahlamohlaka, T. N. et al. A sinter resistant Co Fischer-Tropsch catalyst promoted with Ru and supported on titania encapsulated by mesoporous silica. Appl. Catal. A Gen. 552, 129-137 (2018).

136. Li, H. et al. Evidence of the Encapsulation Model for Strong Metal-Support Interaction under Oxidized Conditions: A Case Study on $\mathrm{TiO}_{\mathrm{x}} / \mathrm{Pt}(111)$ for CO Oxidation by in Situ Wide Spectral Range Infrared Reflection Adsorption Spectroscopy. ACS Catal. 8, 10156-10163 (2018).

137. Weng, Z. \& Zaera, F. Sub-Monolayer Control of Mixed-Oxide Support Composition in Catalysts via Atomic Layer Deposition: Selective Hydrogenation of Cinnamaldehyde Promoted by $\left(\mathrm{SiO}_{2}{ }^{-}\right.$ ALD)-Pt/ $/ \mathrm{Al}_{2} \mathrm{O}_{3}$. ACS Catal. 8, 8513-8524 (2018). 
138. Ro, I. et al. Role of the $\mathrm{Cu}-\mathrm{ZrO}_{2}$ Interfacial Sites for Conversion of Ethanol to Ethyl Acetate and Synthesis of Methanol from $\mathrm{CO}_{2}$ and $\mathrm{H}_{2}$. ACS Catal. 6, 7040-7050 (2016).

139. Moon, S. Y., Naik, B., Jung, C.-H., Qadir, K. \& Park, J. Y. Tailoring metal-oxide interfaces of oxide-encapsulated Pt/silica hybrid nanocatalysts with enhanced thermal stability. Catal. Today 265, 245-253 (2016).

140. Yang, N. \& Bent, S. F. Investigation of inherent differences between oxide supports in heterogeneous catalysis in the absence of structural variations. J. Catal. 351, 49-58 (2017).

141. Kennedy, R. M. et al. Replication of SMSI via ALD: $\mathrm{TiO}_{2}$ Overcoats Increase Pt-Catalyzed Acrolein Hydrogenation Selectivity. Catal. Letters 148, 2223-2232 (2018).

142. Zhang, J. et al. Wet-Chemistry Strong Metal-Support Interactions in Titania Supported Au Catalysts. J. Am. Chem. Soc. 141, 2975-2983 (2019).

143. Wu, L., Li, B. \& Zhao, C. Direct Synthesis of Hydrogen and Dimethoxylmethane from Methanol on Copper/Silica Catalysts with Optimal $\mathrm{Cu}^{+} / \mathrm{Cu}^{0}$ Sites. ChemCatChem 10, 1140-1147 (2018).

144. Xu, C. et al. Interfacing with silica boosts the catalysis of copper. Nat. Commun. 9, 3367 (2018).

145. Matsubu, J. C. et al. Adsorbate-mediated strong metal-support interactions in oxidesupported Rh catalysts. Nat. Chem. 9, 120-127 (2017).

146. Wang, X. et al. Sacrificial Adsorbate Strategy Achieved Strong Metal-Support Interaction of Stable Cu Nanocatalysts. ACS Appl. Energy Mater. 1, 1408-1414 (2018).

147. Aitbekova, A. et al. Low-Temperature Restructuring of $\mathrm{CeO}_{2}-$ Supported $\mathrm{Ru}$ Nanoparticles Determines Selectivity in $\mathrm{CO}_{2}$ Catalytic Reduction. J. Am. Chem. Soc. 140, 13736-13745 (2018).

148. Kleijn, S. E. F., Lai, S. C. S., Koper, M. T. M. \& Unwin, P. R. Electrochemistry of Nanoparticles. Angew. Chem. Int. Ed. 53, 3558-3586 (2014).

149. Vannice, M. A. The Catalytic Synthesis of Hydrocarbons from $\mathrm{H}_{2} / \mathrm{CO}$ Mixtures over the Group VIII Metals: V. The Catalytic Behavior of Silica-Supported Metals. J. Catal. 50, 228-236 (1977). 


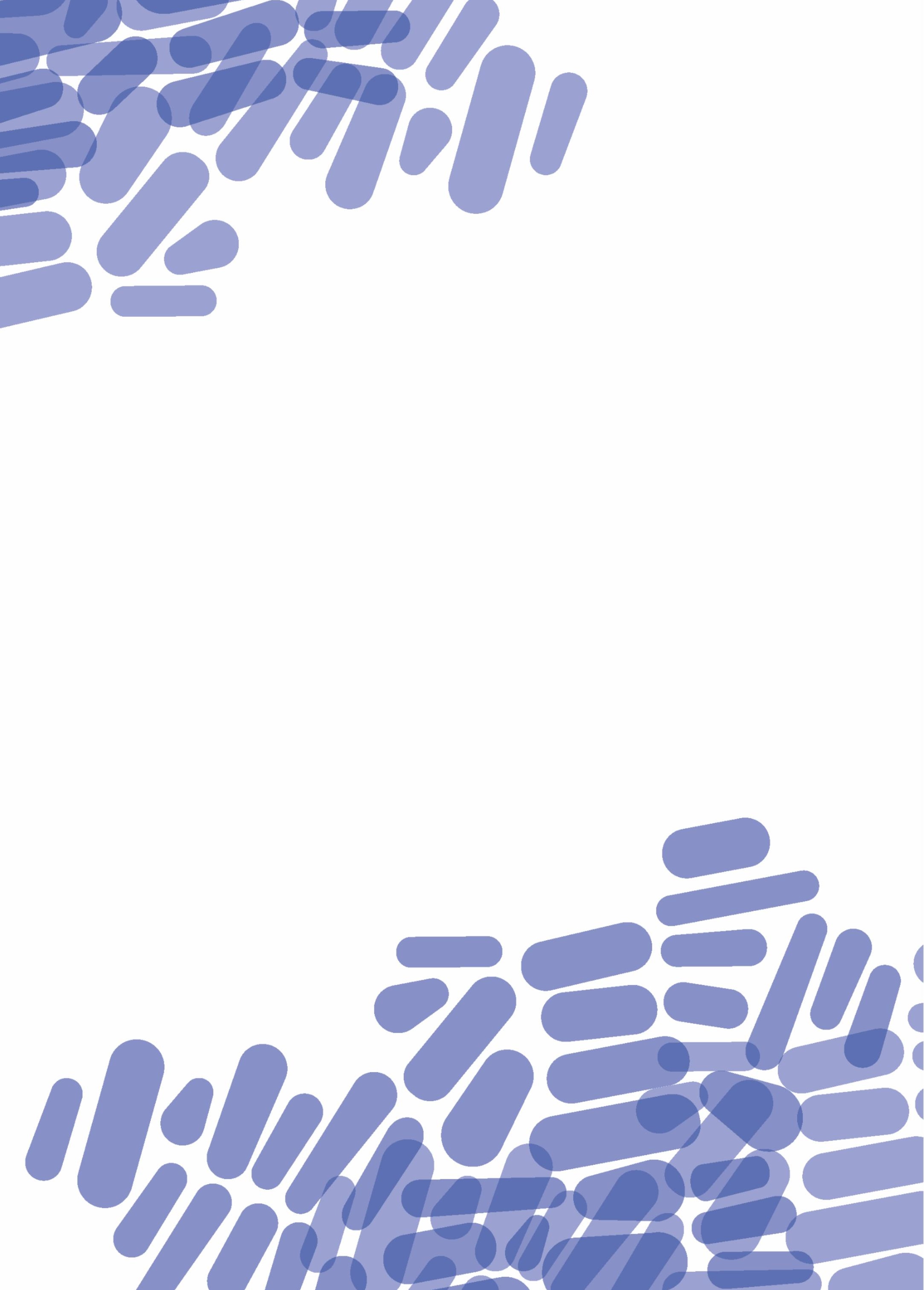




\subsection{Introduction}

Metal particles catalyze major chemical processes, including the synthesis of bulk chemicals and transportation fuels. ${ }^{1-4}$ The catalytic reactions that are involved occur mainly at the metal surface. Therefore, the surface-to-volume ratio of these particles is typically maximized through nanosizing, leading to an adequate exposure of the active sites and efficient utilization of the metal. ${ }^{5,6}$ Supports play a critical role in stabilizing the reactive nanoparticles but also in generating new interphase phenomena, such as electron transfer or shape rearrangement. ${ }^{7-9}$ These phenomena can have ambivalent consequences for the catalytic performance such as promoting activity and selectivity or blocking active sites, which decreases the catalyst efficiency.

A renowned example of support effects occurs when reducible metal oxide supports $\left(\mathrm{TiO}_{2}, \mathrm{Nb}_{2} \mathrm{O}_{5}, \mathrm{CeO}_{2}\right.$, etc. $)$ are partially reduced by hydrogen spillover from the metal nanoparticle ${ }^{10}$, i.e. the so-called strong metal-support interaction (SMSI)..$^{11,12}$ As a result, suboxide species are generated from the support and these may migrate onto the metal nanoparticles surface. ${ }^{13-16}$ In the case of cobalt catalysts for the Fischer-Tropsch (FT) synthesis, the effect of SMSI is twofold. On one hand, the cobalt catalysts benefit from the mildly Lewis acidic character of the suboxide species, as it increases the intrinsic activity (turnover frequency, TOF) and selectivity towards $\mathrm{C}_{5+}$ products. ${ }^{17,18}$ The increased TOF is believed to originate from the interface between the metal and suboxide where the COdissociation rate is enhanced. ${ }^{19-21}$ On the other hand, the catalytic activity can be lowered by a dense overlayer of suboxide species that block the access to the active sites. ${ }^{14}$ The interplay between promotion of the catalytic performance and blockage of active sites is not clear and control over the metal-support interactions may thus provide an opportunity to increase the number of accessible, promoted active sites.

The SMSI state can be reversed by oxidative treatments. However, this reversibility has been mainly studied for noble metals, while reports of base metals are scarce. ${ }^{12,22-24}$ Soled et al. ${ }^{25}$ studied the impact of SMSI on $\mathrm{H}_{2}$-chemisorption for cobalt nanoparticles supported on titania. The $\mathrm{H}_{2}$-uptake was initially suppressed because of site blockage but increased significantly after a reduction-oxidation-reduction (ROR) treatment. This brought $\mathrm{H}_{2}-$ chemisorption and TEM and XRD results into closer agreement, but the effect of ROR on the structure of the cobalt particles as well as its implications for catalysis remained unclear. Besides reversal of the SMSI state, ROR has been applied to regenerate deactivated catalysts through re-dispersion of the active metal ${ }^{26-29}$. The work on catalyst regeneration focused, however, on cobalt catalysts supported on irreducible oxides, such as $\mathrm{Al}_{2} \mathrm{O}_{3}$ and $\mathrm{SiO}_{2}$, in which SMSI effects do not play a role.

In this chapter, we investigate the possibility to tune the metal-support interactions of cobalt supported on reducible metal oxides via reduction-oxidation-reduction treatments with the aim to enhance the catalytic activity in the Fischer-Tropsch synthesis. To this end, $\mathrm{Co} / \mathrm{TiO}_{2}$ and $\mathrm{Co} / \mathrm{Nb}_{2} \mathrm{O}_{5}$ are synthesized and subjected to ROR treatments. Co on irreducible $\alpha-\mathrm{Al}_{2} \mathrm{O}_{3}$ is prepared for comparison. After the first reduction, oxidation above $100{ }^{\circ} \mathrm{C}$ leads to the formation of hollow cobalt oxide particles. Upon re-reduction, the $\mathrm{H}_{2}$-uptake doubles for the reducible metal oxides due to optimization of the SMSI state and not by cobalt re-dispersion, while the $\mathrm{H}_{2}$-uptake decreases for $\alpha-\mathrm{Al}_{2} \mathrm{O}_{3}$. The modified metal- 
support interaction on reducible supports drastically enhances the catalytic activity under industrially relevant FT conditions, effectively doubling the number of available active sites without changing their TOF. Modulation of the metal-support interaction via ROR is thus a promising strategy to boost the catalytic performance and to greatly improve the efficiency of metal catalysts supported on reducible oxides.

\subsection{Experimental}

\subsubsection{Synthesis}

The samples were synthesized by incipient wetness impregnation using aqueous cobalt nitrate solutions. The precursor solutions were prepared by dissolving appropriate amounts of cobalt nitrate hexahydrate (99+\%, Acros Organics) in Milli-Q water to obtain 6 wt. $\%$ cobalt loading on each catalyst. The supports, $\mathrm{TiO}_{2}\left(\mathrm{P} 25\right.$, Evonik, $\mathrm{SA}_{\mathrm{BET}}: 50 \mathrm{~m}^{2} \cdot \mathrm{g}^{-1}$, PV: $0.30 \mathrm{~cm}^{3} \cdot \mathrm{g}^{-1}$ ), $\mathrm{Nb}_{2} \mathrm{O}_{5}$ (pseudo-hexagonal TT-phase, CBMM, SA $A_{\mathrm{BET}}: 9 \mathrm{~m}^{2} \cdot \mathrm{g}^{-1}, \mathrm{PV}$ : $0.06 \mathrm{~cm}^{3} \cdot \mathrm{g}^{-1}$ ) and $\alpha-\mathrm{Al}_{2} \mathrm{O}_{3}$ (BASF, $\mathrm{SA}_{\mathrm{BET}}: 7 \mathrm{~m}^{2} \cdot \mathrm{g}^{-1}, \mathrm{PV}: 0.02 \mathrm{~cm}^{3} \cdot \mathrm{g}^{-1}$ ), were sieved to a grain size of 75-150 $\mu \mathrm{m} . \mathrm{Nb}_{2} \mathrm{O}_{5}$ was obtained from amorphous niobium oxide hydrate (HY-340, $\mathrm{AD} / 4465, \mathrm{CBMM})$ by calcination in a muffle oven at $600{ }^{\circ} \mathrm{C}$ for $4 \mathrm{~h}$.

For a typical impregnation, $2 \mathrm{~g}$ of support was pre-dried at $80^{\circ} \mathrm{C}$ for $1 \mathrm{~h}$ under vacuum. Just before the impregnation, the vacuum was released and a pore filling amount of precursor solution was added dropwise to the support under magnetic and manual stirring. In the case of $\mathrm{Co} / \mathrm{TiO}_{2}, 2 \mathrm{~g}$ of sample was dried and heat treated in fluidized bed mode in a U-shaped glass reactor following a previously reported procedure. ${ }^{30}$ The sample was first dried at $80{ }^{\circ} \mathrm{C}$ for $2 \mathrm{~h}$ and heat treated directly afterwards at $250{ }^{\circ} \mathrm{C}$ for $2 \mathrm{~h}$. Both steps were performed in $1 \mathrm{~L} \cdot \mathrm{min}^{-1} \mathrm{~N}_{2}$ upward flow with a heating ramp of $2^{\circ} \mathrm{C} \cdot \mathrm{min}^{-1}$. $\mathrm{Co} / \mathrm{Nb}_{2} \mathrm{O}_{5}$ and $\mathrm{Co} / \alpha-\mathrm{Al}_{2} \mathrm{O}_{3}$ catalysts were dried for $12 \mathrm{~h}$ at $60{ }^{\circ} \mathrm{C}$ overnight in stagnant air and subsequently calcined in a fixed bed reactor under $\mathrm{N}_{2}$ flow $\left(1 \mathrm{~L} \cdot \mathrm{min}^{-1}\right)$ at $350{ }^{\circ} \mathrm{C}$ for $2 \mathrm{~h}$ with a heating ramp of $3{ }^{\circ} \mathrm{C} \cdot \mathrm{min}^{-1}$. Multiple batches of $2 \mathrm{~g}$ were mixed to obtain a substantial amount of sample. The obtained samples were pressed and sieved to a grain size of 75-150 $\mu \mathrm{m}$.

Ex situ reduction-oxidation (RO) treatments were performed in U-shaped glass reactors. To this end, batches of $500 \mathrm{mg}$ were first reduced at $350{ }^{\circ} \mathrm{C}$ for $2 \mathrm{~h}\left(2{ }^{\circ} \mathrm{C} \cdot \mathrm{min}^{-1}\right)$ in $120 \mathrm{~mL} \cdot \mathrm{min}^{-1}$ of $25 \mathrm{vol} . \% \mathrm{H}_{2}$ in $\mathrm{N}_{2}$ and subsequently cooled to $100{ }^{\circ} \mathrm{C}$. At this point, the reactor was flushed with $\mathrm{N}_{2}$ and cooled down further to $30{ }^{\circ} \mathrm{C}$. The oxidation step followed directly after the reduction and was performed in $95 \mathrm{~mL} \cdot \mathrm{min}^{-1}$ of 5 vol. $\% \mathrm{O}_{2}$ in $\mathrm{N}_{2}$ at various temperatures up to $400{ }^{\circ} \mathrm{C}$ for $2 \mathrm{~h}$ with a $2^{\circ} \mathrm{C} \cdot \mathrm{min}^{-1}$ heating ramp.

\subsubsection{Characterization}

Scanning transmission electron microscopy combined with high-angle annular dark-field (STEM-HAADF) and energy-dispersive X-ray spectroscopy (EDX) analyses were performed on an FEI Talos F200X. This microscope was equipped with a high-brightness field emission gun (X-FEG) and a Super-X G2 EDX detector and operated at $200 \mathrm{kV}$. The EM samples were prepared by suspending the samples in 2-propanol (>99.9\%, SigmaAldrich) using sonication and dropcasting the suspension on a carbon-coated $\mathrm{Cu}$ grid (200 mesh). The cobalt particle size was determined using the iTEM software and at least 200 
particles were analyzed. The reduced and passivated samples were corrected for a $3 \mathrm{~nm}$ $\mathrm{CoO}$ layer and the volume/surface mean diameters $(\mathrm{D}[3,2])$ were calculated.

$\mathrm{H}_{2}$-chemisorption was measured on a Micromeritics ASAP 2020C using 200 mg of sample. The catalysts were reduced in pure $\mathrm{H}_{2}$ prior to the measurement. The pristine samples were reduced for $2 \mathrm{~h}\left(1{ }^{\circ} \mathrm{C} \cdot \mathrm{min}^{-1}\right)$ at $350{ }^{\circ} \mathrm{C}$, while the $\mathrm{RO}$-samples were reduced at $220{ }^{\circ} \mathrm{C}$. After reduction, the samples were evacuated, cooled to $150{ }^{\circ} \mathrm{C}$ and analyzed at that temperature. The metallic surface area and average particle diameter were calculated assuming a cobalt cross-sectional area of $0.0662 \mathrm{~nm}^{2}$, an $\mathrm{H} / \mathrm{Co}$ stoichiometry of 1 and spherical cobalt particles. ${ }^{31}$

Inductively coupled plasma-optical emission spectroscopy (ICP-OES) was performed on a SPECTRO ARCOS. The samples were prepared by extraction of cobalt using aqua regia.

Temperature-programmed reduction (TPR) profiles were measured using a Micromeritics Autochem 2920, equipped with a TCD detector. Typically, $100 \mathrm{mg}$ sample was reduced up to $1000{ }^{\circ} \mathrm{C}$ with $5{ }^{\circ} \mathrm{C} \cdot \mathrm{min}^{-1}$ in a flow of 5 vol. $\% \mathrm{H}_{2}$ in Ar. To determine the degree of reduction, the samples were first reduced in the TPR setup in a flow of pure $\mathrm{H}_{2}$ for $2 \mathrm{~h}$ at $350{ }^{\circ} \mathrm{C}$ or $220^{\circ} \mathrm{C}$ depending of the stage in the ROR treatment and then, the TPR profile was recorded from 50 to $800{ }^{\circ} \mathrm{C}$ in a flow of $5 \mathrm{vol} . \% \mathrm{H}_{2}$ in Ar. The cobalt that had not been reduced was quantified from the areas of the peaks between 250 and $700{ }^{\circ} \mathrm{C}$.

\subsubsection{Fischer-Tropsch synthesis}

The catalytic performance was measured using an Avantium Flowrence 16 parallel reactor setup. Stainless steel reactors (ID $=2 \mathrm{~mm}$ ) were loaded with $75-100 \mathrm{mg}$ of catalyst $(75-$ $150 \mu \mathrm{m})$ and diluted with $100 \mathrm{mg} \mathrm{SiC}(212-425 \mu \mathrm{m})$. Prior to reaction, the catalysts were reduced in situ for $8 \mathrm{~h}\left(1{ }^{\circ} \mathrm{C} \cdot \mathrm{min}^{-1}\right)$ in $25 \mathrm{vol} . \% \mathrm{H}_{2}$ in He at 1 bar. The pristine samples were reduced at $350{ }^{\circ} \mathrm{C}$, while the $\mathrm{RO}$-samples were reduced at $220^{\circ} \mathrm{C}$. After reduction, the temperature was lowered to $180{ }^{\circ} \mathrm{C}\left(3{ }^{\circ} \mathrm{C} \cdot \mathrm{min}^{-1}\right)$ and the pressure was increased to $20 \mathrm{bar}$ under $\mathrm{H}_{2}$ flow. Finally, synthesis gas with $\mathrm{H}_{2} / \mathrm{CO}=2 \mathrm{~V} / \mathrm{V}$ (5 vol. \% He as internal standard) was introduced and the temperature was raised to $220{ }^{\circ} \mathrm{C}$ with $1{ }^{\circ} \mathrm{C} \cdot \mathrm{min}^{-1}$. The product stream was analyzed online with an Agilent 7890A GC. The hydrocarbon products were analyzed on an Agilent J\&W PoraBOND Q column connected to an FID. The permanent gasses were separated on a ShinCarbon ST column and quantified using a TCD.

The activity of the catalysts was expressed as CO conversion, cobalt time yield (CTY) and turnover frequency (TOF). The CO conversion was defined according to (Equation 7.1), CTY according to (Equation 7.2) and TOF according to (Equation 7.3). In these equations, $\mathrm{X}_{\mathrm{CO}}$ is the $\mathrm{CO}$ conversion, $\mathrm{F}_{\mathrm{CO} \text {,in }}$ is the flow of $\mathrm{CO}$ into the reactor and $\mathrm{F}_{\mathrm{CO} \text {,out }}$ is the flow of $\mathrm{CO}$ in the product stream. $\mathrm{V}_{\mathrm{m}}$ denotes the molar volume, $\mathrm{m}_{\mathrm{Co}}$ the mass of cobalt in the reactor, $\mathrm{N}_{\mathrm{A}}$ is the Avogadro constant, $\mathrm{d}_{\mathrm{Co}}$ the cross-sectional area of a cobalt atom $\left(0.0662 \mathrm{~nm}^{2}\right.$ according to ref. ${ }^{31}$ ) and $\mathrm{SA}_{\mathrm{Co}}$ is the cobalt specific surface area as obtained by $\mathrm{H}_{2}-$ chemisorption.

The selectivity towards light hydrocarbon products with carbon number $\mathrm{n}$ (with $1 \leq \mathrm{n} \leq 4$ ) was calculated as in (Equation 7.4) and the selectivity towards the heavier fraction $(\mathrm{n} \geq 5)$ as in (Equation 7.5) In this case, $\mathrm{S}_{\mathrm{Cn}}$ represents the selectivity towards hydrocarbon product of a specific carbon number and $\mathrm{F}_{\mathrm{Cn}}$ is the flow of the corresponding hydrocarbon product. 


$$
\begin{aligned}
& \mathrm{X}_{\mathrm{CO}}=\left(\mathrm{F}_{\mathrm{CO}, \text { in }}-\mathrm{F}_{\mathrm{CO}, \text { out }}\right) \cdot \mathrm{F}_{\mathrm{CO}, \text { in }}{ }^{-1} \\
& \mathrm{CTY}=\mathrm{F}_{\mathrm{CO}, \text { in }} \cdot \mathrm{X}_{\mathrm{CO}} \cdot \mathrm{V}_{\mathrm{m}}{ }^{-1} \cdot \mathrm{m}_{\mathrm{CO}}{ }^{-1} \\
& \mathrm{TOF}=\mathrm{CTY} \cdot \mathrm{N}_{\mathrm{A}} \cdot \mathrm{d}_{\mathrm{CO}} \cdot \mathrm{SA}_{\mathrm{CO}}{ }^{-1} \\
& \mathrm{~S}_{\mathrm{Cl}-\mathrm{C} 4}=\mathrm{F}_{\mathrm{Cn}} \cdot \mathrm{n} \cdot\left(\mathrm{F}_{\mathrm{CO}, \text { in }} \cdot \mathrm{X}_{\mathrm{CO}}\right)^{-1} \\
& \mathrm{~S}_{\mathrm{C} 5+}=1-\mathrm{S}_{\mathrm{Cl}-\mathrm{C} 4}
\end{aligned}
$$

(Equation 7.5)

\subsection{Results and discussion}

\subsubsection{Reduction}

Cobalt particles supported on reducible oxides $\mathrm{TiO}_{2}$ and $\mathrm{Nb}_{2} \mathrm{O}_{5}$, and on irreducible oxide $\alpha-$ $\mathrm{Al}_{2} \mathrm{O}_{3}$ were prepared by incipient wetness impregnation followed by drying and thermal treatment. The nominal cobalt loading was $6 \mathrm{wt} . \%$ and experimentally determined loadings are given in Appendix E, Table E1. $\mathrm{H}_{2}$-chemisorption analysis was performed on the pristine samples, which were reduced at $350{ }^{\circ} \mathrm{C}$ prior to the measurement (referred to as $\mathrm{R}$-samples). After $\mathrm{H}_{2}$-chemisorption, the samples were passivated by exposure to air at room temperature and subsequently analyzed using scanning transmission electron microscopy-energy-dispersive X-ray spectroscopy (STEM-EDX, Figure 7.1a-c). The cobalt particles were rather uniformly distributed over $\mathrm{TiO}_{2}$ and $\mathrm{Nb}_{2} \mathrm{O}_{5}$ while the spatial

\begin{tabular}{|c|c|c|c|}
\hline Sample & $\begin{array}{l}\text { Particle diameter } \\
\mathrm{D}[3,2] \pm \text { standard } \\
\text { deviation }(\mathrm{nm})^{\mathrm{a}}\end{array}$ & $\begin{array}{l}\text { Theoretical } \\
\mathrm{H}_{2}-\text { uptake } \\
\left(\mathrm{\mu mol}_{\mathrm{H}_{2}} \cdot \mathrm{g}_{\mathrm{Co}}{ }^{-1}\right)^{\mathrm{a}}\end{array}$ & $\begin{array}{l}\text { Experimental } \\
\mathrm{H}_{2} \text {-uptake } \\
\left(\mu \mathrm{mol}_{\mathrm{H}_{2}} \cdot \mathrm{g}_{\mathrm{Co}}{ }^{-1}\right)^{\mathrm{b}} \\
\end{array}$ \\
\hline \multicolumn{4}{|l|}{ R-samples } \\
\hline $\mathrm{R}-\mathrm{TiO}_{2}$ & $11 \pm 3.4$ & 787 & 341 \\
\hline $\mathrm{R}-\mathrm{Nb}_{2} \mathrm{O}_{5}$ & $19 \pm 10$ & 437 & 265 \\
\hline $\mathrm{R}-\mathrm{Al}_{2} \mathrm{O}_{3}$ & $13 \pm 6.5$ & 666 & 631 \\
\hline \multicolumn{4}{|c|}{ RO200R-samples } \\
\hline $\mathrm{RO} 200 \mathrm{R}-\mathrm{TiO}_{2}$ & $10 \pm 3.8$ & 865 & 783 \\
\hline $\mathrm{RO} 200 \mathrm{R}-\mathrm{Nb}_{2} \mathrm{O}_{5}$ & $18 \pm 7.9$ & 470 & 657 \\
\hline $\mathrm{RO} 200 \mathrm{R}-\mathrm{Al}_{2} \mathrm{O}_{3}$ & $21 \pm 11$ & 412 & 499 \\
\hline
\end{tabular}
distribution was more heterogeneous on $\alpha-\mathrm{Al}_{2} \mathrm{O}_{3}$.

Table 7.1. The results of cobalt particle size analyses using STEM-EDX and $\mathrm{H}_{2}$-chemisorption for the pristine samples after reduction at $350{ }^{\circ} \mathrm{C}\left(\mathrm{R}\right.$-samples) and the samples after reduction at $350{ }^{\circ} \mathrm{C}$, oxidation at $200{ }^{\circ} \mathrm{C}$ and reduction at $220^{\circ} \mathrm{C}$ (RO200R-samples).

a Derived from STEM-EDX

${ }^{\mathrm{b}}$ Derived from $\mathrm{H}_{2}$-chemisorption

$\mathrm{H}_{2}$-chemisorption and STEM-EDX results for the R-samples are given in Table 7.1. The theoretical $\mathrm{H}_{2}$-uptake was calculated for cobalt particles using the surface-average particle diameter derived from STEM-EDX images. This theoretical value was compared to the experimental uptake measured by $\mathrm{H}_{2}$-chemisorption. In the case of $\mathrm{R}-\mathrm{TiO}_{2}$ and $\mathrm{R}-\mathrm{Nb}_{2} \mathrm{O}_{5}$, a substantial discrepancy in $\mathrm{H}_{2}$-uptake was observed between both methodologies. This is 
explained by partial coverage of the metallic cobalt surface by suboxide species leading to suppressed uptake in the $\mathrm{H}_{2}$-chemisorption measurement. It is important to note that the total amount of suboxide species is probably low, because no overlayers could be detected with STEM-EDX nor with in situ TEM of $\mathrm{Co} / \mathrm{Nb}_{2} \mathrm{O}_{5}$ under reducing conditions in a previous study. ${ }^{32}$ No large discrepancy between the theoretical and experimental $\mathrm{H}_{2}$-uptake of $\mathrm{R}$ $\mathrm{Al}_{2} \mathrm{O}_{3}$ was observed, because suboxides were not formed.

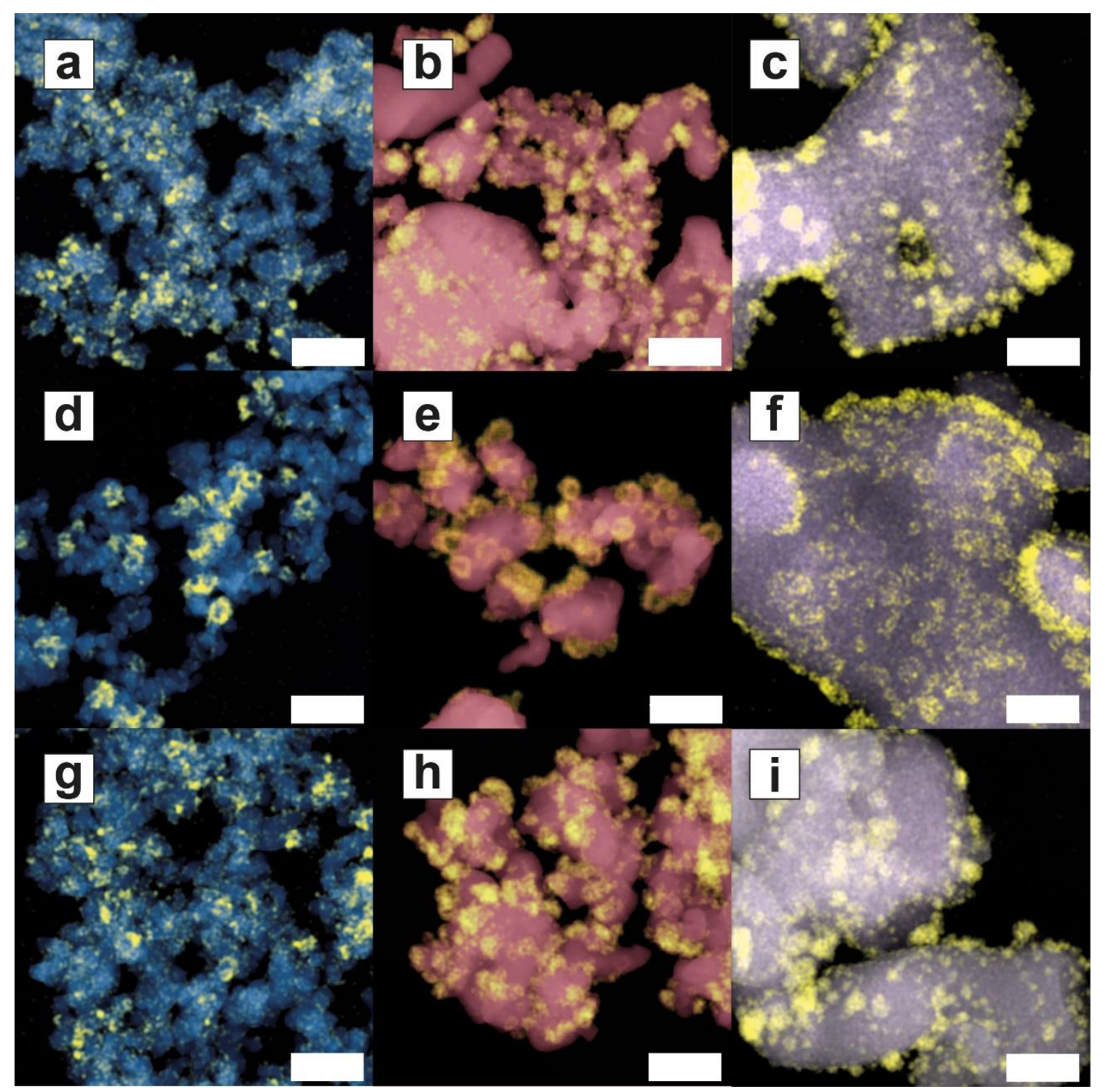

Figure 7.1. Electron microscopy images of the samples upon reduction-oxidation-reduction treatments. STEM-EDX mapping of $\mathrm{Co} / \mathrm{TiO}_{2}, \mathrm{Co} / \mathrm{Nb}_{2} \mathrm{O}_{5}$ and $\mathrm{Co} / \alpha-\mathrm{Al}_{2} \mathrm{O}_{3}$ at various stages of the $\mathrm{ROR}$ treatment. Co is depicted in yellow, Ti in blue, $\mathrm{Nb}$ in red and $\mathrm{Al}$ in violet. a, b, c Samples after reduction at $350^{\circ} \mathrm{C}$ and passivation (R-Samples); $\mathrm{d}$, e, $\mathrm{f}$ samples after reduction at $350{ }^{\circ} \mathrm{C}$ and oxidation at $200{ }^{\circ} \mathrm{C}$ (RO200-samples); $\mathrm{g}, \mathrm{h}, \mathbf{i}$ samples after reduction at $350{ }^{\circ} \mathrm{C}$, oxidation at $200{ }^{\circ} \mathrm{C}$ and reduction at $220^{\circ} \mathrm{C}$ (RO200R-samples) and passivation. The scale bar corresponds to $100 \mathrm{~nm}$. Additional electron microscopy results for these samples can be found in Appendix E, Figures E1-3. 


\subsubsection{Reduction-oxidation}

After the first reduction treatment at $350{ }^{\circ} \mathrm{C}$, the R-samples were subjected to an oxidative treatment at temperatures varying between $30{ }^{\circ} \mathrm{C}$ and $400{ }^{\circ} \mathrm{C}$ (referred to as RO30- to RO400-samples). The temperature-programmed reduction (TPR) profiles of pristine $\mathrm{Co}_{3} \mathrm{O}_{4} / \mathrm{Nb}_{2} \mathrm{O}_{5}$ and $\mathrm{RO} 30-\mathrm{Nb}_{2} \mathrm{O}_{5}$ to $\mathrm{RO} 400-\mathrm{Nb}_{2} \mathrm{O}_{5}$ are shown in Figure 7.2a. The pristine sample displayed two-step, consecutive reduction from $\mathrm{Co}_{3} \mathrm{O}_{4}$ to $\mathrm{CoO}\left(280-300{ }^{\circ} \mathrm{C}\right)$ and from $\mathrm{CoO}$ to $\mathrm{Co}\left(300-400{ }^{\circ} \mathrm{C}\right)$. Samples that had been reduced and oxidized at temperatures up to $100{ }^{\circ} \mathrm{C}$ showed only one sharp reduction peak at $230{ }^{\circ} \mathrm{C}$ corresponding to the reduction of $\mathrm{CoO}$. Therefore, the second reduction of these samples can be performed at substantially lower temperature than the first one. Based on the amount of consumed $\mathrm{H}_{2}$, we found that some metallic cobalt was still present on those samples, probably as a metallic core covered by a $\mathrm{CoO}$ layer. Further increasing the oxidation temperature to $150{ }^{\circ} \mathrm{C}$ led to broadening of the narrow peak $\left(\mathrm{RO} 150-\mathrm{Nb}_{2} \mathrm{O}_{5}\right)$ and eventually resulted in a two-step reduction profile, associated with the formation of $\mathrm{Co}_{3} \mathrm{O}_{4}$ during oxidation $\left(\mathrm{RO} 200-\mathrm{Nb}_{2} \mathrm{O}_{5}\right)$. Upon oxidation at $400{ }^{\circ} \mathrm{C}$, the reduction profile resembled that of the pristine $\mathrm{CO}_{3} \mathrm{O}_{4} / \mathrm{Nb}_{2} \mathrm{O}_{5}$ sample.

a

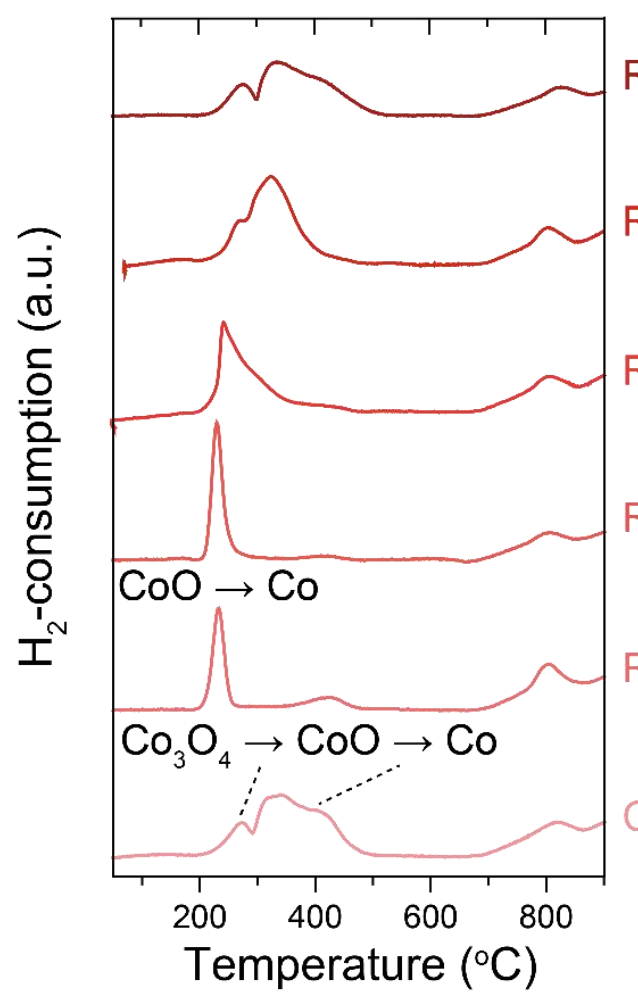

b
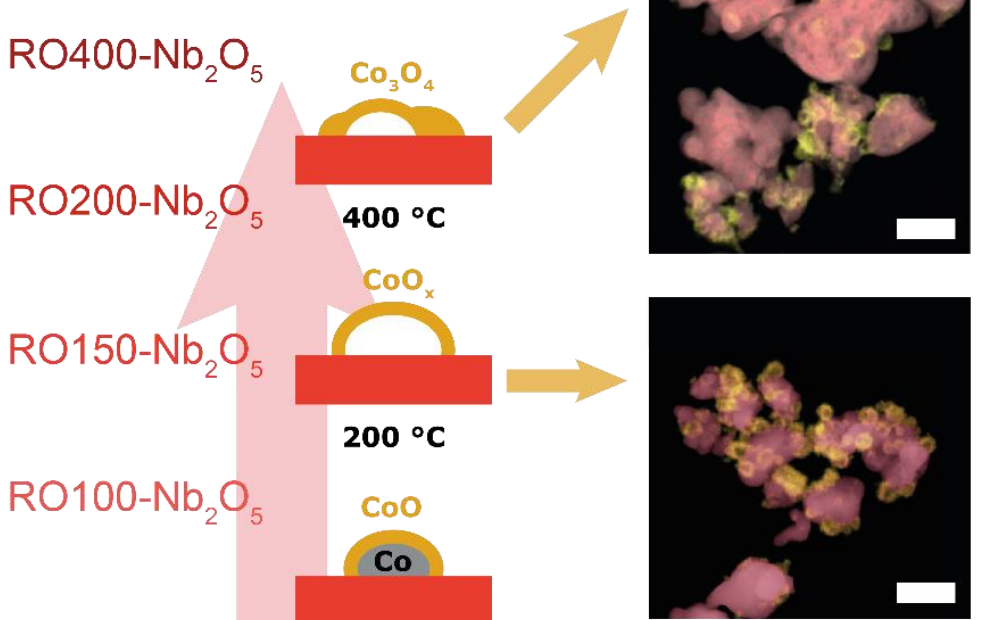

$\mathrm{CO}_{3} \mathrm{O}_{4} / \mathrm{Nb}_{2} \mathrm{O}$ $30^{\circ} \mathrm{C}$

$\mathrm{Co}_{3} \mathrm{O}_{4}$

$\mathrm{Nb}_{2} \mathrm{O}_{5}$

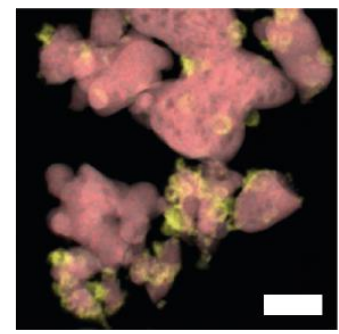

Figure 7.2. The effect of the oxidation temperature on the structure of $\mathrm{Co} / \mathrm{Nb}_{2} \mathrm{O}_{5}$. a Temperatureprogrammed reduction profiles, $\mathbf{b}$ schematic illustration and $\mathbf{c} S T E M-E D X$ images of $\mathrm{Co} / \mathrm{Nb}_{2} \mathrm{O}_{5}$ after reduction and oxidation at various temperatures $\left(30-400^{\circ} \mathrm{C}\right)$. Co is depicted in yellow, $\mathrm{Nb}$ in red. The scale bar corresponds to $100 \mathrm{~nm}$. 
The RO-samples were characterized by STEM-EDX mapping. Samples oxidized at low temperatures appeared unchanged (Figure 7.2b,c). However, at oxidation temperatures of $200{ }^{\circ} \mathrm{C}$, hollow cobalt oxide particles were observed (Figure 7.1d-f). The formation of these hollow particles was ascribed to the Kirkendall effect. ${ }^{33-35}$ During oxidation, an initial oxide layer forms around the metallic particle and at higher temperature, the smaller $\mathrm{Co}^{2+} \mathrm{Or}^{\mathrm{Co}^{3+}}$ cations diffuse outwards through the oxide layer faster than the larger $\mathrm{O}^{2}$ anions diffuse inwards, forming shells of cobalt oxide leaving behind empty cores. At an oxidation temperature of $400{ }^{\circ} \mathrm{C}$, the hollow cobalt oxide particles got distorted and agglomerated.

\subsubsection{Reduction-oxidation-reduction}

The samples after an ROR cycle were characterized by $\mathrm{H}_{2}$-chemisorption (Figure 7.3a). The second reduction of the ROR treatment was performed at $220^{\circ} \mathrm{C}$, whereas the R-samples had been reduced at $350^{\circ} \mathrm{C}$. For the reducible oxides, the $\mathrm{H}_{2}$-uptake significantly increased at oxidation temperatures above $100{ }^{\circ} \mathrm{C}$. In the case of $\mathrm{TiO}_{2}$, it changed from $341 \mu \mathrm{mol}_{\mathrm{H} 2} \cdot \mathrm{g}_{\mathrm{Co}}$ ${ }^{1}$ for $\mathrm{R}-\mathrm{TiO}_{2}$ to $783 \mu \mathrm{mol}_{\mathrm{H} 2} \cdot \mathrm{g}_{\mathrm{Co}}{ }^{-1}$ for $\mathrm{RO} 200 \mathrm{R}-\mathrm{TiO}_{2}$. Similarly, for $\mathrm{Nb}_{2} \mathrm{O}_{5}$ it increased from

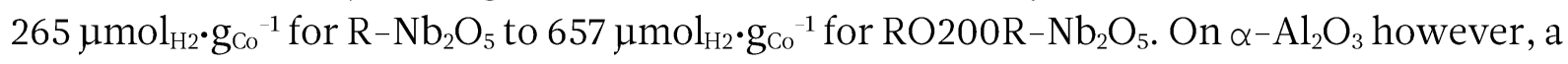
substantial decrease of $\mathrm{H}_{2}$-uptake was observed after ROR treatments. The degree of reduction (DOR) was determined by TPR directly after the last reduction for all $\mathrm{R}$ - and RO200R-samples and for RO400R- $\mathrm{Nb}_{2} \mathrm{O}_{5}$ (Appendix E, Figure E4 and Appendix E, Table E2). The DOR for the R-samples was $97-98 \%$ and decreased slightly to $84-95 \%$ after ROR. Because these changes were minor, the $\mathrm{H}_{2}$-chemisorption results were not corrected for the DOR.

The particle sizes of the RO200R-samples were investigated using STEM-EDX (Figure 7.1g-i). The spatial distribution of the particles over the supports was not markedly different from that in the R-samples (Figure 7.1a-c). Furthermore, the average size of the cobalt particles was similar to that of the R-samples, despite the significantly different values obtained by $\mathrm{H}_{2}$-chemisorption (Figure 7.3b-d, Table 7.1). In fact, the theoretical $\mathrm{H}_{2}-$ uptake now approached the values of the experimental $\mathrm{H}_{2}$-uptake.

On $\mathrm{TiO}_{2}$, the increase in $\mathrm{H}_{2}$-uptake with a similar average particle size implied that $\mathrm{ROR}$ did not induce substantial re-dispersion of cobalt. Rather, the ROR treatment resulted in a higher fraction of accessible metallic cobalt surface through a decrease of the surface coverage by suboxide species (Figure 7.3e). On $\mathrm{Nb}_{2} \mathrm{O}_{5}$, however, the situation is more complex, because the experimental $\mathrm{H}_{2}$-uptake after ROR was higher than the theoretical one. The additional $\mathrm{H}_{2}$-uptake could be rationalized in two ways. First, re-dispersion of few large cobalt particles could have formed small particles, which would be undetectable by STEM-EDX. Second, hydrogen could have spilled over to $\mathrm{Nb}_{2} \mathrm{O}_{5}$, in line with the fact that $\mathrm{Nb}_{2} \mathrm{O}_{5}$ has been proven to facilitate hydrogen storage. ${ }^{36,37}$ On $\alpha-\mathrm{Al}_{2} \mathrm{O}_{3}$, the same Kirkendall behavior occurred at oxidation temperatures above $100{ }^{\circ} \mathrm{C}$ (Figure 7.1f), but here the $\mathrm{H}_{2}$-uptake decreased on all ROR-samples due to particle growth.

ROR has been reported to regenerate deactivated catalysts through re-dispersion of the active metal via the Kirkendall effect. ${ }^{26-29}$ However, the work on catalyst regeneration focused on cobalt catalysts supported on irreducible oxides, such as $\mathrm{Al}_{2} \mathrm{O}_{3}$ and $\mathrm{SiO}_{2}$, in which SMSI effects do not play a role. Furthermore, we ascribe the absence of net redispersion in our samples to different initial cobalt particle sizes. The particles in our R- 
$\mathrm{TiO}_{2}(11 \mathrm{~nm})$ and $\mathrm{R}-\mathrm{Al}_{2} \mathrm{O}_{3}(13 \mathrm{~nm})$ were substantially smaller than those reported in literature $(22 \mathrm{~nm}) .{ }^{29}$ According to Sadasivan et al..$^{38}$, only cobalt particles above a certain critical size give rise to re-dispersion upon ROR treatments.
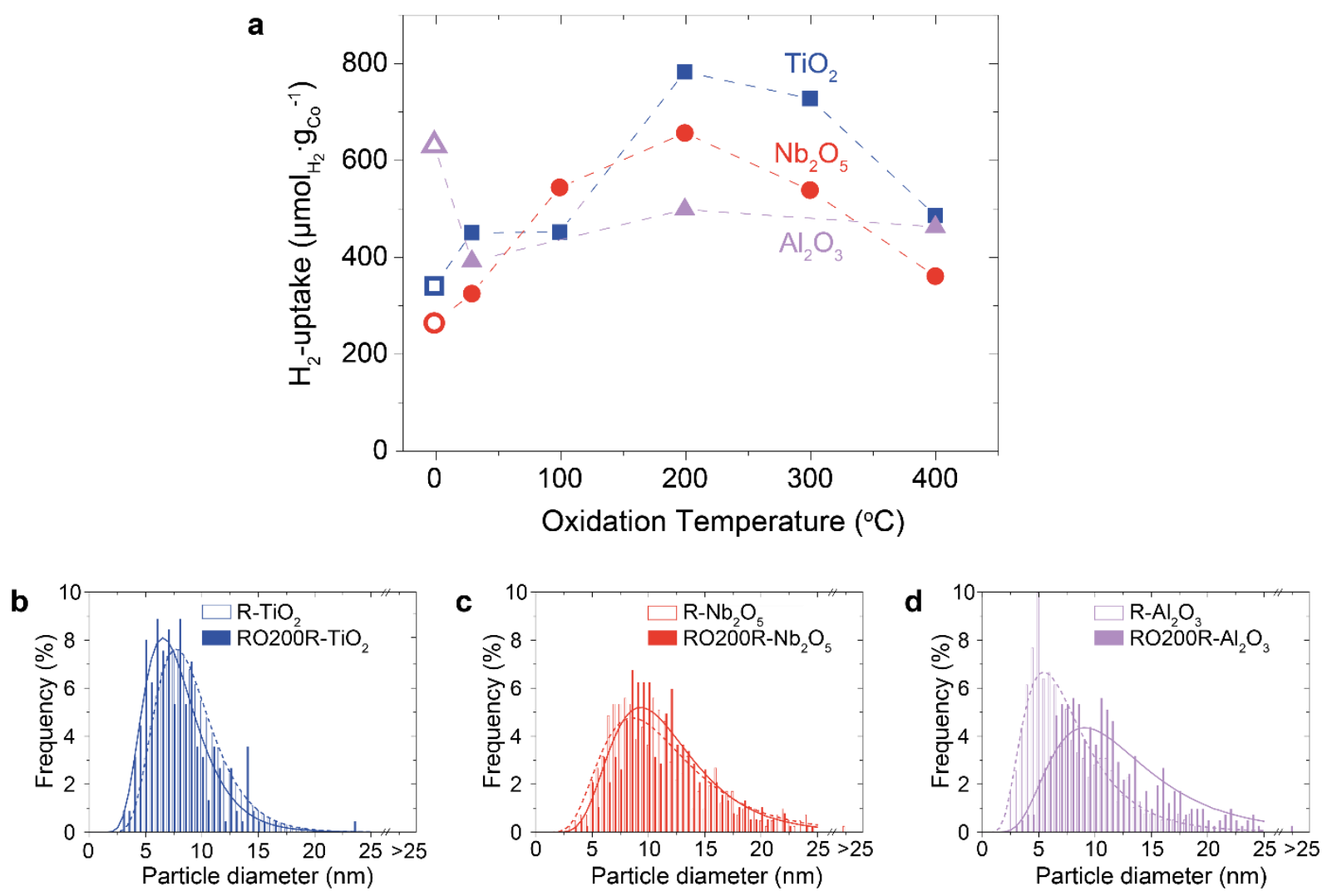

e

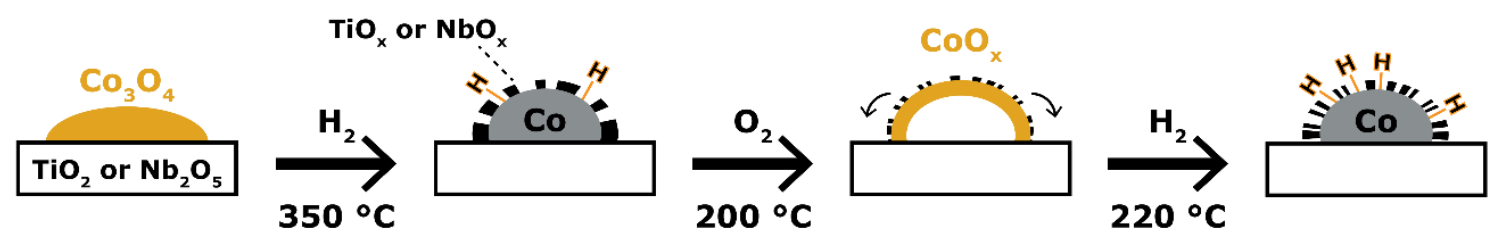

Figure 7.3. The effect of ROR treatments on the exposed cobalt surface area and particle size. a Hydrogen uptake for $\mathrm{Co} / \mathrm{TiO}_{2}$ (blue squares), $\mathrm{Co} / \mathrm{Nb}_{2} \mathrm{O}_{5}$ (red circles) and $\mathrm{Co} / \alpha-\mathrm{Al}_{2} \mathrm{O}_{3}$ (violet triangles) after various reduction-oxidation-reduction treatments, as determined by $\mathrm{H}_{2}-$ chemisorption. The results of the pristine samples after reduction at $350{ }^{\circ} \mathrm{C}$ (open symbols) are included at 0 on the $\mathbf{x}$-axis. $\mathbf{b}, \mathbf{c}, \mathbf{d}$ Particle size distributions of cobalt supported on $\mathrm{TiO}_{2}, \mathrm{Nb}_{2} \mathrm{O}_{5}$ and $\alpha-\mathrm{Al}_{2} \mathrm{O}_{3}$, respectively, after reduction at $350{ }^{\circ} \mathrm{C}$ (open bars) and after reduction at $350{ }^{\circ} \mathrm{C}$ followed by oxidation at $200^{\circ} \mathrm{C}$ and reduction at $220^{\circ} \mathrm{C}$ (solid filled bars), as determined by STEM-EDX. e Schematic illustration of the effect of the ROR treatment on cobalt supported on reducible oxides.

The trends observed with $\mathrm{H}_{2}$-chemisorption for the reducible oxides can be understood based on the previously discussed results. The Kirkendall effect was most likely key in tuning the SMSI during ROR, because at oxidation temperatures up to $100{ }^{\circ} \mathrm{C}$, where this effect was not observed, the increase in $\mathrm{H}_{2}$-uptake was minor. The $\mathrm{H}_{2}$-uptake only increased significantly when the Kirkendall effect took place. On the other side of the 
optimum, at an oxidation temperature of $400{ }^{\circ} \mathrm{C}$, the high temperature induced particle growth from 10 up to $15 \mathrm{~nm}$ for $\mathrm{RO} 400 \mathrm{R}-\mathrm{TiO}_{2}$, as determined using STEM-EDX (Appendix E, Figure E5). This reduced the metallic surface area and counteracted the positive effect of the ROR treatment on the SMSI.

a

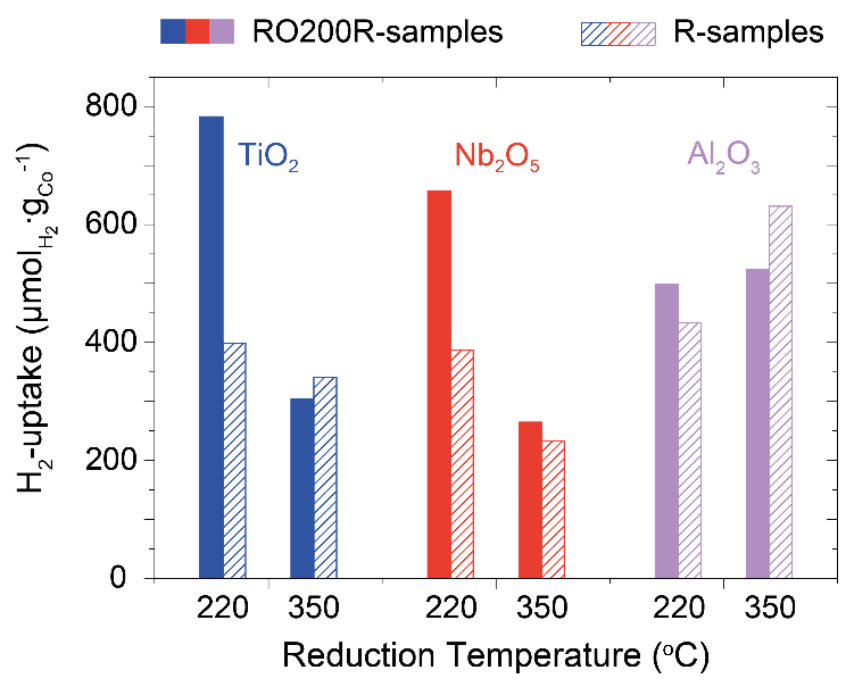

b

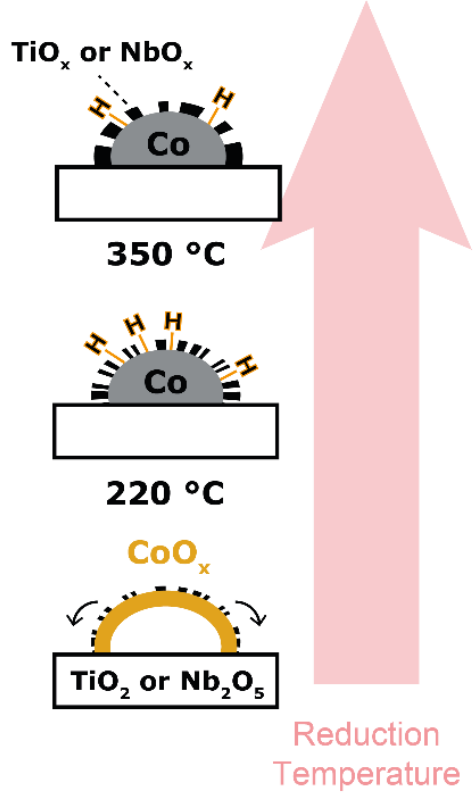

Figure 7.4. The influence of the temperature of the second reduction on the exposed cobalt surface area. a Hydrogen uptake as a function of the second reduction temperature. Dashed bars correspond to the pristine samples after reduction at 220 or $350^{\circ} \mathrm{C}$ and solid filled bars correspond to the samples after reduction at $350^{\circ} \mathrm{C}$ followed by oxidation at $200^{\circ} \mathrm{C}$ and reduction at 220 or $350^{\circ} \mathrm{C}$. b Schematic illustration of the effect of the reduction temperature on catalysts supported on reducible oxides.

Further indications for the optimization of SMSI effects through ROR were found when the second reduction was performed at a higher temperature (Figure 7.4). The $\mathrm{H}_{2}$-uptake decreased for the $\mathrm{RO} 200 \mathrm{R}-\mathrm{TiO}_{2}$ and $\mathrm{RO} 200 \mathrm{R}-\mathrm{Nb}_{2} \mathrm{O}_{5}$ samples with increasing reduction temperature. At $350{ }^{\circ} \mathrm{C}$, the $\mathrm{H}_{2}$-uptake values returned to the values of the initial $\mathrm{R}$ samples that were reduced once. Apparently, the mild temperature of the second reduction was essential to keep the concentration or mobility of the suboxide species low, while it was sufficiently high to reduce cobalt oxide to metallic cobalt. Additionally, the $\mathrm{H}_{2}$-uptakes of the R-samples at reduction temperatures of 220 and $350{ }^{\circ} \mathrm{C}$ were comparable. The substantial increase in $\mathrm{H}_{2}$-uptake for the RO200R-samples was thus not merely caused by reduction at lower temperature. In contrast, the $\mathrm{H}_{2}$-uptake of the $\mathrm{Co} / \alpha-\mathrm{Al}_{2} \mathrm{O}_{3}$ catalysts increased slightly at higher reduction temperatures.

\subsubsection{Fischer-Tropsch synthesis}

The catalytic activity of $\mathrm{Co} / \mathrm{TiO}_{2}$ and $\mathrm{Co} / \mathrm{Nb}_{2} \mathrm{O}_{5}$ in $\mathrm{FT}$ after ROR followed the same trend as $\mathrm{H}_{2}$-chemisorption, i.e. increased activity with the optimum at $200{ }^{\circ} \mathrm{C}$ (Figure $7.5 \mathrm{a}$ ). 
Notably, the activity doubled on $\mathrm{TiO}_{2}$ and $\mathrm{Nb}_{2} \mathrm{O}_{5}$ as a result of the RO200R treatment and the enhancement was stable over at least $100 \mathrm{~h}$ time-on-stream (Appendix E, Figure E6). As expected, the activity of $\mathrm{Co} / \alpha-\mathrm{Al}_{2} \mathrm{O}_{3}$ was not enhanced, indicating that the positive effect of the ROR treatment was specific for catalysts supported on reducible oxides. The $\mathrm{C}_{5_{+}}$-selectivity for all catalysts was $85-89 \%$ and did not change significantly as a result of the ROR treatments (Appendix E, Table E3).

The observed cobalt-weight-based activities of RO200R-TiO $\left(16 \cdot 10^{-5} \mathrm{~mol}_{\mathrm{CO}} \cdot \mathrm{g}_{\mathrm{Co}}{ }^{-1} \cdot \mathrm{s}^{-1}\right)$ and RO200R- $\mathrm{Nb}_{2} \mathrm{O}_{5}\left(8.9 \cdot 10^{-5} \mathrm{~mol} \mathrm{CO} \cdot \mathrm{g}_{\mathrm{Co}}{ }^{-1} \cdot \mathrm{s}^{-1}\right)$ are exceptionally high. Compared to literature ${ }^{18,32,39-43}$, activities in this range are normally only achieved by promoting a cobaltbased catalyst with noble metals (Appendix E, Figure E7). Tuning of the metal-support interactions by ROR or other pretreatments might thus provide an attractive alternative to promotion with expensive and scarce noble metals.

a

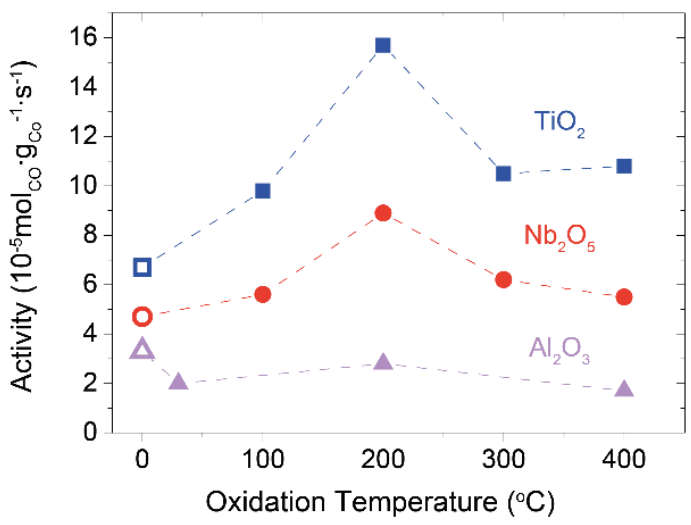

b

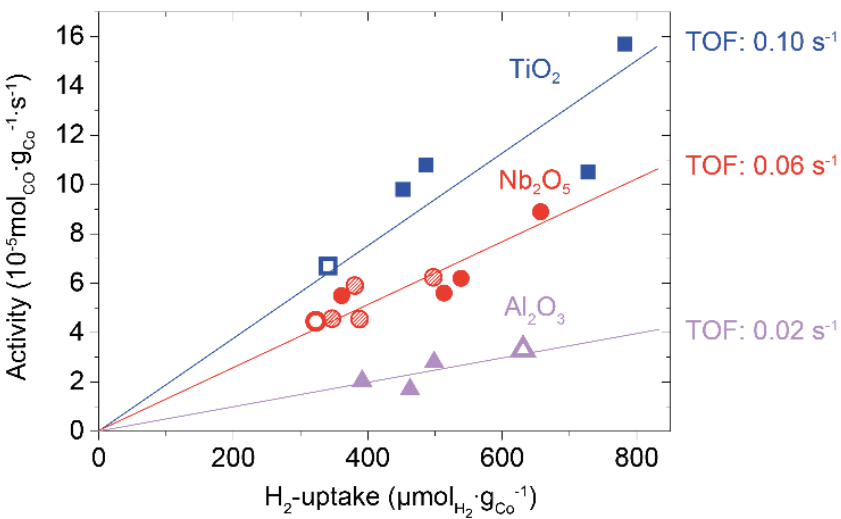

Figure 7.5. The effect of the ROR treatment on the catalytic activity in the Fischer-Tropsch synthesis. FT was performed at $20 \mathrm{bar}, 220^{\circ} \mathrm{C}, \mathrm{H}_{2} / \mathrm{CO}=2 \mathrm{~V} / \mathrm{V}$, GHSV $=1000-13000 \mathrm{~h}^{-1}$, time-onstream $=90-100 \mathrm{~h}, \mathrm{CO}$ conv. $=15-34 \%\left(\mathrm{TiO}_{2}\right), 17-25 \%\left(\mathrm{Nb}_{2} \mathrm{O}_{5}\right), 8-12 \%\left(\mathrm{Al}_{2} \mathrm{O}_{3}\right)$. a Cobalt - weight based catalytic activity of $\mathrm{Co} / \mathrm{TiO}_{2}$ (blue squares), $\mathrm{Co} / \mathrm{Nb}_{2} \mathrm{O}_{5}$ (red circles) and $\mathrm{Co} / \mathrm{Al}_{2} \mathrm{O}_{3}$ (violet triangles) after various reduction-oxidation-reduction treatments. The activity of the pristine samples after reduction at $350{ }^{\circ} \mathrm{C}$ (R-samples, open symbols) is included at 0 on the $\mathrm{x}$-axis. b Cobaltweight-based activity as a function of hydrogen uptake for the R-samples (open symbols) and the samples after reduction at $350^{\circ} \mathrm{C}$ followed by oxidation between $30-400{ }^{\circ} \mathrm{C}$ and reduction at either $220{ }^{\circ} \mathrm{C}$ (filled symbols) or at $250{ }^{\circ} \mathrm{C}$ (dashed symbols); values for the turnover frequencies (TOF) have been calculated from the slopes of the lines.

The ROR treatments increased the catalytic activity of $\mathrm{Co} / \mathrm{TiO}_{2}$ and $\mathrm{Co} / \mathrm{Nb}_{2} \mathrm{O}_{5}$ with respect to the $\mathrm{R}$-samples. The increase in activity was proportional to the $\mathrm{H}_{2}$-uptake (Figure 7.5b), indicating that the surface-specific activity or turnover frequency (TOF, based on activity and $\mathrm{H}_{2}$-chemisorption data; see SI for details) was constant and more surface sites with identical characteristics became available. The opposite was observed with irreducible $\alpha-\mathrm{Al}_{2} \mathrm{O}_{3}$, where the activity decreased after ROR and less surface sites were available albeit at constant TOF. Clearly, the TOF was support-dependent and increased in the order $\alpha-\mathrm{Al}_{2} \mathrm{O}_{3}<\mathrm{Nb}_{2} \mathrm{O}_{5}<\mathrm{TiO}_{2}$. 
The high TOF values for the reducible oxides show that Co surface sites were promoted by $\mathrm{TiO}_{\mathrm{x}}$ or $\mathrm{NbO}_{\mathrm{x}}$ in the $\mathrm{R}$ - and $\mathrm{ROR}$-samples. In addition, the TOF was independent of ROR, meaning that ROR did not change the nature of the promoted active sites. Therefore, the enhanced cobalt-weight-based activity of $\mathrm{Co} / \mathrm{TiO}_{2}$ and $\mathrm{Co} / \mathrm{Nb}_{2} \mathrm{O}_{5}$ implied that the number of promoted active sites increased as a result of ROR. Furthermore, the effect of the second reduction temperature on the activity is included as well in Figure 7.5b for $\mathrm{Co} / \mathrm{Nb}_{2} \mathrm{O}_{5}$. Reduction at $250{ }^{\circ} \mathrm{C}$ gave only a moderate increase in $\mathrm{H}_{2}$-chemisorption and a corresponding increase in activity, while reduction at $220{ }^{\circ} \mathrm{C}$ doubled the $\mathrm{H}_{2}$-uptake and activity.

Although the TOF of cobalt catalysts has been reported to be support independent ${ }^{39,44}$, recent publications have shown that the promoting effect of the support can be substantial. ${ }^{17,18,21,32}$ This promoting effect on the TOF has been related to the Lewis acidity of the metal oxide: an increase in acidity leads to an increase in TOF. ${ }^{17,18}$ The Lewis acid character of oxidic materials originates from exposed metal centers ${ }^{45}$, meaning that the structural arrangement and oxidation state of the ions will determine their acid strength. ${ }^{46}$ Jeong et al. ${ }^{47}$ have recently reported an acidity scale based on the intramolecular charge transfer energy between organic molecules and several metal oxides (AppendixE, Table E4). The values reported for the supports used in our work do predict higher TOF for $\mathrm{TiO}_{2}$ and $\mathrm{Nb}_{2} \mathrm{O}_{5}$ compared to $\mathrm{Al}_{2} \mathrm{O}_{3}$, but the comparison between $\mathrm{TiO}_{2}$ and $\mathrm{Nb}_{2} \mathrm{O}_{5}$ requires further analysis. As mentioned before, Lewis acidity depends on the oxidation state of the cation, thus values for lower oxidation states in suboxides (e.g. $\mathrm{Ti}^{3+}, \mathrm{Nb}^{4+} \mathrm{Or}^{\mathrm{Nb}^{3+}}$ ) gain importance for reducible metal oxides as shown in the work of Boffa et al. ${ }^{21,48}$ Because the oxidation state and structural arrangement of the relevant suboxides are not completely established, the formal Lewis acidity remains uncertain.

\subsection{Conclusion}

Reduction-oxidation-reduction (ROR) treatments were investigated as a tool to tune metal-support interactions in supported cobalt catalysts to improve their performance in the Fischer-Tropsch synthesis. Reduction of cobalt oxide supported on reducible $\left(\mathrm{TiO}_{2}\right.$ and $\left.\mathrm{Nb}_{2} \mathrm{O}_{5}\right)$ and irreducible $\left(\alpha-\mathrm{Al}_{2} \mathrm{O}_{3}\right)$ oxides followed by controlled oxidation led to hollow cobalt oxide particles via the Kirkendall effect. A second reduction resulted in a twofold increase in $\mathrm{H}_{2}$-uptake for the $\mathrm{TiO}_{2}-$ and $\mathrm{Nb}_{2} \mathrm{O}_{5}$-supported samples and a decrease for the $\alpha-$ $\mathrm{Al}_{2} \mathrm{O}_{3}$-supported sample. This effect was attributed to modulation of the metal-support interactions rather than re-dispersion of the cobalt phase. The modified metal-support interactions greatly increased the catalytic activity in the Fischer-Tropsch synthesis, as the activity doubled on $\mathrm{TiO}_{2}$ and $\mathrm{Nb}_{2} \mathrm{O}_{5}$ and decreased slightly on $\alpha-\mathrm{Al}_{2} \mathrm{O}_{3}$. This increased activity was caused by more accessible cobalt surface sites as revealed by the constant turnover frequency, while preserving the full promotional effect of the $\mathrm{TiO}_{\mathrm{x}}$ or $\mathrm{NbO}_{\mathrm{x}}$ species. The more efficient utilization of the cobalt and support interphase led to exceptionally active catalysts. Tailoring of metal-support interactions via ROR treatments is thus an effective method to improve the activity of metal catalysts supported on reducible oxides. 


\section{Acknowledgements}

Companhia Brasileira de Metalurgia e Mineração (CBMM), Shell Global Solutions and the Netherlands Association for Scientific Research (NWO) are thanked for financial support. K.P.d.J. acknowledges the European Research Council, EU FP7 ERC Advanced Grant no. 338846. Robson Monteiro (CBMM), Rogério Ribas (CBMM) and Peter Munnik (Shell Global Solutions) are acknowledged for useful discussions. Wouter Lamme, Jessi van der Hoeven, Nynke Krans and Mark Meijerink (STEM-EDX) and Helen de Waard (ICP-OES) are acknowledged for the measurements indicated.

\section{References}

1. Rayment, T., Schlögl, R., Thomas, J. M. \& Ertl, G. Structure of the ammonia synthesis catalyst. Nature 315, 311-313 (1985).

2. Torres Galvis, H. M. et al. Supported Iron Nanoparticles as Catalysts for Sustainable Production of Lower Olefins. Science 335, 835-838 (2012).

3. Zečević, J., Vanbutsele, G., de Jong, K. P. \& Martens, J. A. Nanoscale intimacy in bifunctional catalysts for selective conversion of hydrocarbons. Nature 528, 245-248 (2015).

4. Behrens, M. et al. The Active Site of Methanol Synthesis over $\mathrm{Cu} / \mathrm{ZnO} / \mathrm{Al}_{2} \mathrm{O}_{3}$ Industrial Catalysts. Science 336, 893-898 (2012).

5. Campbell, C. T., Parker, S. C. \& Starr, D. E. The Effect of Size-Dependent Nanoparticle Energetics on Catalyst Sintering. Science 298, 811-814 (2002).

6. Munnik, P., de Jongh, P. E. \& de Jong, K. P. Recent Developments in the Synthesis of Supported Catalysts. Chem. Rev. 115, 6687-6718 (2015).

7. Lykhach, Y. et al. Counting electrons on supported nanoparticles. Nat. Mater. 15, 284-288 (2016).

8. Divins, N. J., Angurell, I., Escudero, C., Pérez-Dieste, V. \& Llorca, J. Influence of the support on surface rearrangements of bimetallic nanoparticles in real catalysts. Science 346, 620-623 (2014).

9. Kitano, M. et al. Ammonia synthesis using a stable electride as an electron donor and reversible hydrogen store. Nat. Chem. 4, 934-940 (2012).

10. Karim, W. et al. Catalyst support effects on hydrogen spillover. Nature 541, 68-71 (2017).

11. Tauster, S. J., Fung, S. C. \& Garten, R. L. Strong Metal-Support Interactions. Group 8 Noble Metals Supported on $\mathrm{TiO}_{2}$. J. Am. Chem. Soc. 100, 170-175 (1978).

12. Tauster, S. J., Fung, S. C., Baker, R. T. \& Horsley, J. A. Strong Interactions in Supported-Metal Catalysts. Science 211, 1121-1125 (1981).

13. Matsubu, J. C. et al. Adsorbate-mediated strong metal-support interactions in oxidesupported Rh catalysts. Nat. Chem. 9, 120-127 (2017).

14. Zhang, S. et al. Dynamical Observation and Detailed Description of Catalysts under Strong Metal-Support Interaction. Nano Lett. 16, 4528-4534 (2016).

15. de la Peña O’Shea, V. A., Álvarez Galván, M. C., Platero Prats, A. E., Campos-Martin, J. M. \& Fierro, J. L. G. Direct evidence of the SMSI decoration effect: the case of $\mathrm{Co} / \mathrm{TiO}_{2}$ catalyst. Chem. Commun. 47, 7131-7133 (2011).

16. $\mathrm{Xu}, \mathrm{M}$. et al. $\mathrm{TiO}_{2-\mathrm{x}}-$ Modified Ni Nanocatalyst with Tunable Metal-Support Interaction for Water-Gas Shift Reaction. ACS Catal. 7, 7600-7609 (2017). 
17. Johnson, G. R. \& Bell, A. T. Effects of Lewis acidity of metal oxide promoters on the activity and selectivity of Co-based Fischer-Tropsch synthesis catalysts. J. Catal. 338, 250-264 (2016).

18. Prieto, G. et al. Cobalt-Catalyzed Fischer-Tropsch Synthesis: Chemical Nature of the Oxide Support as a Performance Descriptor. ACS Catal. 5, 3323-3335 (2015).

19. Maitlis, P. M. \& Zanotti, V. The role of electrophilic species in the Fischer-Tropsch reaction. Chem. Commun. 0, 1619-1634 (2009).

20. Vannice, M. A. Hydrogenation of CO and Carbonyl Functional Groups. Catal. Today 12, 255-267 (1992).

21. Boffa, A. B., Lin, C., Bell, A. T. \& Somorjai, G. A. Lewis acidity as an explanation for oxide promotion of metals: implications of its importance and limits for catalytic reactions. Catal. Letters 27, 243-249 (1994).

22. Foger, K. Reactions of n-Butane and Neopentane on Titania-Supported Iridium Catalysts. $J$. Catal. 418, 406-418 (1982).

23. Braunschweig, E. J., Logan, A. D., Datye, A. K. \& Smith, D. J. Reversibility of Strong MetalSupport Interactions on $\mathrm{Rh} / \mathrm{TiO}_{2}$. J. Catal. 118, 227-237 (1989).

24. Pan, C.-J. et al. Tuning/exploiting Strong Metal-Support Interaction (SMSI) in Heterogeneous Catalysis. J. Taiwan Inst. Chem. Eng. 74, 154-186 (2017).

25. Soled, S. L. et al. Control of metal dispersion and structure by changes in the solid-state chemistry of supported cobalt Fischer-Tropsch catalysts. Top. Catal. 26, 101-109 (2003).

26. Weststrate, C. J. et al. Cobalt Fischer-Tropsch Catalyst Regeneration: The Crucial Role of the Kirkendall Effect for Cobalt Redispersion. Top. Catal. 54, 811-816 (2011).

27. Saib, A. M. et al. Fundamental understanding of deactivation and regeneration of cobalt Fischer-Tropsch synthesis catalysts. Catal. Today 154, 271-282 (2010).

28. Saib, A. M. et al. Fundamental Science of Cobalt Catalyst Oxidation and Reduction Applied to the Development of a Commercial Fischer-Tropsch Regeneration Process. Ind. Eng. Chem. Res. 53,1816-1824 (2014).

29. Hauman, M. M. et al. Re-dispersion of Cobalt on a Model Fischer-Tropsch Catalyst During Reduction-Oxidation-Reduction Cycles. ChemCatChem 4, 1411-1419 (2012).

30. Eschemann, T. O. \& de Jong, K. P. Deactivation Behavior of $\mathrm{Co} / \mathrm{TiO}_{2}$ Catalysts during FischerTropsch Synthesis. ACS Catal. 5, 3181-3188 (2015).

31. Reuel, R. C. \& Bartholomew, C. H. The Stoichiometries of $\mathrm{H}_{2}$ and CO Adsorptions on Cobalt: Effects of Support and Preparation. J. Catal. 85, 63-77 (1984).

32. den Otter, J. H., Yoshida, H., Ledesma, C., Chen, D. \& de Jong, K. P. On the superior activity and selectivity of $\mathrm{PtCo} / \mathrm{Nb}_{2} \mathrm{O}_{5}$ Fischer Tropsch catalysts. J. Catal. 340, 270-275 (2016).

33. Yin, Y. et al. Formation of Hollow Nanocrystals Through the Nanoscale Kirkendall Effect. Science 304, 711-714 (2004).

34. Chernavskii, P. A., Pankina, G. V, Zaikovskii, V. L., Peskov, N. V \& Afanasiev, P. Formation of Hollow Spheres upon Oxidation of Supported Cobalt Nanoparticles. J. Phys. Chem. C112, 95739578 (2008).

35. González-Carballo, J. M., Sadasivan, S., Landon, P. \& Tooze, R. P. Synthesis of Cobalt Nanodumbbells and their Thermal Stability under $\mathrm{H}_{2}, \mathrm{H}_{2} / \mathrm{CO}$ and $\mathrm{O}_{2}$ Atmospheres. Mater. Charact. 118, 519-526 (2016). 
36. Barkhordarian, G., Klassen, T. \& Bormann, R. Fast hydrogen sorption kinetics of nanocrystalline $\mathrm{Mg}$ using $\mathrm{Nb}_{2} \mathrm{O}_{5}$ as catalyst. Scr. Mater. 49, 213-217 (2003).

37. Dolci, F., Di Chio, M., Baricco, M. \& Giamello, E. Niobium pentoxide as promoter in the mixed $\mathrm{MgH}_{2} / \mathrm{Nb}_{2} \mathrm{O}_{5}$ system for hydrogen storage: A multitechnique investigation of the $\mathrm{H}_{2}$ uptake. $J$. Mater. Sci. 42, 7180-7185 (2007).

38. Sadasivan, S., Bellabarba, R. M. \& Tooze, R. P. Size dependent reduction-oxidation-reduction behaviour of cobalt oxide nanocrystals. Nanoscale 5, 11139-11146 (2013).

39. Iglesia, E. Design, synthesis, and use of cobalt-based Fischer-Tropsch synthesis catalysts. Appl. Catal. A Gen. 161, 59-78 (1997).

40. Sun, X. et al. Manufacture of highly loaded silica-supported cobalt Fischer-Tropsch catalysts from a metal organic framework. Nat. Commun. 8, 1680 (2017).

41. Nabaho, D., Niemantsverdriet, J. W., Claeys, M. \& Van Steen, E. Hydrogen spillover in the Fischer-Tropsch synthesis: An analysis of gold as a promoter for cobalt-alumina catalysts. Catal. Today 275, 27-34 (2016).

42. Eschemann, T. O., Oenema, J. \& de Jong, K. P. Effects of noble metal promotion for $\mathrm{Co} / \mathrm{TiO}_{2}$ Fischer-Tropsch catalysts. Catal. Today 261, 60-66 (2016).

43. Lögdberg, S. et al. Effect of water on the space-time yield of different supported cobalt catalysts during Fischer-Tropsch synthesis. Appl. Catal. A Gen. 393, 109-121 (2011).

44. Iglesia, E., Soled, S. L. \& Fiato, R. A. Fischer-Tropsch Synthesis on Cobalt and Ruthenium. Metal Dispersion and Support Effects on Reaction Rate and Selectivity. J. Catal. 137, 212-224 (1992).

45. Corma, A. Inorganic Solid Acids and Their Use in Acid-Catalyzed Hydrocarbon Reactions. Chem. Rev. 95, 559-614 (1995).

46. Kreissl, H. T. et al. Structural Studies of Bulk to Nanosize Niobium Oxides with Correlation to Their Acidity. J. Am. Chem. Soc. 139, 12670-12680 (2017).

47. Jeong, N. C., Lee, J. S., Tae, E. L., Lee, Y. J. \& Yoon, K. B. Acidity scale for metal oxides and Sanderson's electronegativities of lanthanide elements. Angew. Chemie Int. Ed. 47, 1012810132 (2008).

48. Boffa, A. B., Lin, C., Bell, A. T. \& Somorjai, G. A. Promotion of $\mathrm{CO}$ and $\mathrm{CO}_{2}$ Hydrogenation over Rh by Metal Oxides: The Influence of Oxide Lewis Acidity and Reducibility. J. Catal. 149, 149158 (1994). 



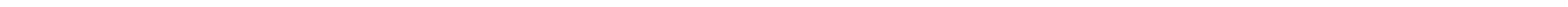




\section{Summary}

Heterogeneous catalysis is an essential technology for the production of fuels and chemicals. The demand for fossil fuels, and in particular liquid fuels for transportation, is expected to grow in the next two decades while limitations on $\mathrm{CO}_{2}$ emissions are likely to become a major constraint for the production. Gas-to-liquids technology has the potential to reduce the overall $\mathrm{CO}_{2}$ footprint of liquid fuels and to produce ultra clean fuels as an additional benefit.

The Fischer-Tropsch (FT) synthesis, i.e. the catalytic conversion of $\mathrm{CO}$ and $\mathrm{H}_{2}$ to longchain hydrocarbons, is an important component of the gas-to-liquids process. Nanosized cobalt particles on a support material are often used to catalyze FT and the structure of the nanoparticles can strongly affect their catalytic performance. For example, Co nanoparticle size and crystal structure effects have already been reported.

Further studies of cobalt structure performance relationships in FT could be facilitated with well-defined model catalysts based on cobalt nanocrystals (Co-NC), prepared using colloidal techniques. This provides, in principle, control over the size, shape, and composition of the Co-NC. However, subsequent attachment of the Co-NC to a support and activation are equally important for catalytic applications, but these are relatively unexplored research fields for non-noble metals. The aim of the research described in this thesis is to study the preparation and application of Co-NC-based model catalyst in FT to establish cobalt structure-performance relationships.

In Chapter 2, we investigated the effect of oxidative treatments of $\mathrm{Co}-\mathrm{NC}$ on their attachment to carbon nanotubes (CNT) and activation for the Fischer-Tropsch synthesis. Attachment of $\varepsilon$-cobalt NC resulted in clustering of the NC and low activity. After lowtemperature oxidation of the $\mathrm{NC}$ to $\mathrm{CoO}$, the $\mathrm{NC}$ were attached uniformly to the CNT. The ligands were readily removed during in situ reduction and high activity was obtained. In fact, it was possible to fully activate a NC-based cobalt catalyst for FT using this mild procedure. High-temperature oxidation to $\mathrm{Co}_{3} \mathrm{O}_{4}$ led to more pronounced particle growth during in situ reduction or FT and therefore the catalytic activity was lower. A severe oxidative treatment was thus unnecessary and even counter-productive. Overall, mild, low-temperature oxidation was thus the preferred treatment for assembly and activation of the Co-NC-based catalysts.

In Chapter 3, we investigated the impact of CNT support oxidation on the structure and performance of cobalt-based catalysts in FT. Well-defined model catalysts on CNT and oxidized CNT (oxCNT) with $10 \mathrm{wt} \%$ Co loading were synthesized through attachment of colloidal $6 \mathrm{~nm} \mathrm{CoO}-\mathrm{NC}$. The $\mathrm{CoO}-\mathrm{NC}$ on pristine CNT were reduced completely to Co-NC and grew to $11 \mathrm{~nm}$ during reduction. In contrast, on oxCNT, only $\sim 50 \% \mathrm{CoO}$ reduction was observed and the $\mathrm{NC}$ remained $7 \mathrm{~nm}$, indicating a stronger metal-support interaction on oxCNT. Co-NC/CNT were exceptionally active in FT with a turnover frequency (TOF) of $150 \cdot 10^{-3} \mathrm{~s}^{-1}$ and relatively stable. In comparison, the TOF on oxCNT was only $63 \cdot 10^{-3} \mathrm{~s}^{-1}$ and the catalyst deactivated more extensively. Using in situ XRD, oxidation of CNT was found to result in $32 \%$ less crystalline, metallic cobalt, which coincided with a $35 \%$ lower initial TOF. The stronger deactivation with $\mathrm{Co}-\mathrm{NC}$ on oxidized CNT could not be explained by 
particle growth, NC restructuring, or Co oxidation during FT, leaving increased deposition of carbonaceous species as a possible cause.

In Chapter 4, we investigated the preparation of Co- $\mathrm{NC}$ supported on $\mathrm{TiO}_{2}$ and $\mathrm{SiO}_{2}$ and evaluated their catalytic performance in FT. 3-12 nm Co-NC were synthesized by a hot-injection method, oxidized at low temperature to restrict magnetic interactions and subsequently attached to $\mathrm{TiO}_{2}$ or $\mathrm{SiO}_{2}$. The catalytic activity of most $\mathrm{TiO}_{2}$-supported samples was in line with that of a $\mathrm{Co} / \mathrm{TiO}_{2}$ sample prepared by impregnation of cobalt nitrate, proving that relevant catalytic data was obtained with these model catalysts. The cobalt-weight based activity of $3 \mathrm{~nm} \mathrm{Co}-\mathrm{NC}$ on $\mathrm{TiO}_{2}$ was five-fold lower than expected and this was partially ascribed to loss of cobalt to the support. Furthermore, the $\mathrm{TiO}_{2}-$ supported Co particles and $3 \mathrm{~nm} \mathrm{Co-NC}$ on $\mathrm{SiO}_{2}$ sintered during reduction and FT, while 6 and $9 \mathrm{~nm} \mathrm{Co}-\mathrm{NC}$ on $\mathrm{SiO}_{2}$ were stable. We proposed that the interaction between $\mathrm{Co}(\mathrm{O})$ and $\mathrm{TiO}_{2}$ was responsible for the decreased stability of the Co-NC.

In Chapter 5, we investigated the synthesis of disk-shaped Co-NC, their deposition on a support and the effect of their anisotropy on FT. Disk-shaped hcp Co-NC were synthesized simultaneously with spherical \& Co-NC. After oxidation to CoO, these NC were deposited on low surface area silica. Electron microscopy analysis revealed that numerically $66 \%$ of the deposited $\mathrm{CoO}-\mathrm{NC}$ were disks and $34 \%$ were spheres. After in situ reduction of the $\mathrm{CoO}-\mathrm{NC}$ to $\mathrm{Co}$, the Fischer-Tropsch activity of the disk-containing catalyst was only half that of a catalyst containing only spherical NC. Interestingly, the catalysts were stable and the size and shape of the NC were retained even for the diskshaped Co-NC. XPS showed that most of the amine ligands were removed during activation/FT and in situ XRD revealed that the crystal structure of both catalysts during FT was similar, with $\mathrm{NC}$ with 3-5 nm polycrystalline domains and approximately $65 \%$ hcp Co and 21-32 \% fcc Co. Based on these experiments, we tentatively propose that changing the Co-NC shape from spheres to disks reduced FT activity because of enhanced occurrence of hcp (0001) facets.

In Chapter 6, we reviewed recent literature on tuning of metal-support interaction (MSI), focussing on strategies to enhance catalytic performance. This topic has received much attention in recent years, however, a systematic rationalization of the field is lacking due to the great diversity in catalysts, reactions and MSI modification strategies. In this review, we covered and categorized the recent progress in MSI tuning strategies to enhance catalytic performance for various reactions. Furthermore, we quantified recently reported productivity enhancements in $\mathrm{C}_{1}$ chemistry resulting from MSI control. Our analysis showed that up to 15-fold productivity enhancement has been achieved and that MSI has large impact for metal nanoparticles smaller than $4 \mathrm{~nm}$.

In Chapter 7, we investigated reduction-oxidation-reduction (ROR) treatments as a tool to tune MSI in supported cobalt catalysts to improve their FT performance. Reduction of cobalt oxide supported on reducible $\left(\mathrm{TiO}_{2}\right.$ and $\left.\mathrm{Nb}_{2} \mathrm{O}_{5}\right)$ and irreducible $\left(\alpha-\mathrm{Al}_{2} \mathrm{O}_{3}\right)$ oxides followed by controlled oxidation led to hollow cobalt oxide particles via the Kirkendall effect. A second reduction resulted in a twofold increase in $\mathrm{H}_{2}$-uptake for the $\mathrm{TiO}_{2}$ - and $\mathrm{Nb}_{2} \mathrm{O}_{5}$-supported samples and a decrease for the $\alpha-\mathrm{Al}_{2} \mathrm{O}_{3}$-supported sample. This effect was attributed to modulation of MSI rather than re-dispersion of the cobalt phase. The 
modified MSI doubled the catalytic FT activity of $\mathrm{Co}$ on $\mathrm{TiO}_{2}$ and $\mathrm{Nb}_{2} \mathrm{O}_{5}$ while it decreased slightly for Co on $\alpha-\mathrm{Al}_{2} \mathrm{O}_{3}$. This increase in activity was caused by more accessible cobalt surface sites as revealed by the constant TOF, while preserving the full promotional effect of the $\mathrm{TiO}_{\mathrm{x}}$ or $\mathrm{NbO}_{\mathrm{x}}$ species. The more efficient utilization of the cobalt and support interphase led to exceptionally active FT catalysts.

\section{Concluding remarks}

The first aim was to develop a method to prepare supported model cobalt catalysts relevant for the Fischer-Tropsch synthesis. Oxidation of as-synthesized Co-NC at room temperature was identified as preferred treatment for uniform distribution of $\mathrm{Co}-\mathrm{NC}$ on a support and their efficient activation, while minimizing the impact on NC properties. In chapters 2 to 5 we showed the versatility of this approach, as it was applied to prepare Co$\mathrm{NC}$ on CNT (untreated and oxidized) and on the oxidic supports $\mathrm{TiO}_{2}$ and $\mathrm{SiO}_{2}$. The main drawback of this approach for model catalyst synthesis is the inevitable loss of control over the crystal structure of the metal due to oxidation. We anticipate that this strategy of on purpose oxidizing as-synthesized $\mathrm{NC}$ at low temperature to circumvent magnetic interactions and consequent clustering of NC might prove useful for uniformly attaching other ferromagnetic metal NC to supports as well.

At present, these NC-based catalysts are suitable mainly for model studies and not for industrial application, because of their typically delicate and expensive synthesis procedure. Very few NC-based catalysts are therefore commercially available. However, relevance for industry comes from the insights that can potentially be gained from the model studies that can now be performed. Several examples of such studies are presented in this thesis, in which the synthesized NC-based catalysts are employed to establish structure-performance relationships.

First, functional groups and defects on CNT strengthened the metal-support interaction and made the catalysts more resistant to sintering, but also reduced catalytic intrinsic activity and selectivity through lowering of the amount crystalline Co and increasing the rate of deactivation. Notably, the activity of the Co-NC on pristine CNT was exceptionally high with a TOF of $150 \cdot 10^{-3} \mathrm{~s}^{1}$. Nevertheless, open questions still remain, such as the type of oxygen-containing functionalities on CNT, the location of the amorphous Co and the cause for the observed deactivation on oxCNT. If the understanding of these metalsupport interactions progresses, it can hopefully be extrapolated to design optimal interfaces on industrially more relevant supports.

Second, the resistance of Co-NC to particle growth was higher on $\mathrm{SiO}_{2}$ than on $\mathrm{TiO}_{2}$. Growth strongly depended on NC size with $3 \mathrm{~nm}$ being less stable than $6 \mathrm{~nm}$ and all initial sizes growing to approximately $12 \mathrm{~nm}$ on $\mathrm{TiO}_{2}$. The implication of these observations is that cobalt particles smaller than $12 \mathrm{~nm}$ are not stable on this particular type of $\mathrm{TiO}_{2}$, so it is questionable whether it would be worthwhile to target nanoparticles smaller than $12 \mathrm{~nm}$ for this catalyst.

Third, an effect of NC shape on FT performance was observed. Lower FT activity was obtained with disk-shaped than with spherical Co-NC, which was tentatively ascribed to increased exposure of hcp (0001) facets on the disks. Remarkably, the disk shape of the NC 
endured the harsh FT conditions for over $100 \mathrm{~h}$. This means that, in principle, other shapes have the potential to improve FT performance, provided that the exposure of more active facets is favored.

The advanced studies that can be performed with NC-based catalysts are not limited to the examples described in this thesis. Other interesting cobalt structure-FT performance studies could be performed using pre-formed NC, such as investigating promoter effects through precisely controlling the amount and location of promoters, or investigating catalyst stability and NC growth mechanisms through synthesis of a catalyst with a bimodal NC size distribution from mixed batches of NC. In addition, this approach could be extended in an analogous manner to other cobalt-catalyzed reactions or to NC of related metals.

In addition to model studies with $\mathrm{Co}-\mathrm{NC}$, we investigated the interplay between metal nanoparticles and supports in more detail. From a literature survey, it was concluded that tuning of metal-support interactions can lead to up to 15 times enhancement of productivity. In addition, we found that ROR treatments doubled the activity of cobalt on reducible supports by mitigating $\mathrm{TiO}_{\mathrm{x}}-$ or $\mathrm{NbO}_{\mathrm{x}}$-overlayer coverage. This could be interesting from an application point of view, because it would allow process intensification using a relatively straightforward pre-treatment and the effect seems stable, at least during the $100 \mathrm{~h}$ that were investigated here.

Overall, the potential of Co-NC as model catalysts for FT as well as regulation of metalsupport interactions for enhanced catalytic performance have been explored. The conclusions from the work described herein could lead to improved catalytic performance through rational catalyst design. 



\section{Samenvatting}

Heterogene katalyse is een essentiële technologie voor de productie van brandstoffen en chemicaliën. Naar verwachting zal de vraag naar fossiele brandstoffen, en in het bijzonder vloeibare brandstoffen voor transport, de komende jaren toenemen terwijl restricties op $\mathrm{CO}_{2}$-emmissies waarschijnlijk een serieuze beperking voor de productie zullen vormen. Het zogenaamde 'gas-to-liquids' (GTL) proces heeft de potentie om de netto $\mathrm{CO}_{2}-$ voetafdruk van vloeibare brandstoffen te reduceren en ultraschone brandstoffen te produceren als bijkomend voordeel.

De Fischer-Tropschsynthese (FT), d.w.z. de gekatalyseerde omzetting van $\mathrm{CO}$ en $\mathrm{H}_{2}$ tot lange koolwaterstofketens, is een belangrijke component van GTL. Kobaltdeeltjes van enkele nanometers groot op een dragermateriaal worden vaak gebruikt als katalysator voor FT en de structuur van de nanodeeltjes kan hun katalytische prestaties sterk beï nvloeden. Effecten van de grootte en kristalstructuur van de kobaltnanodeeltjes zijn bijvoorbeeld al gerapporteerd.

Verdere studies naar de relaties tussen kobaltstructuur en FT-prestaties zouden gefaciliteerd kunnen worden met goed gedefinieerde modelkatalysatoren op basis van kobaltnanokristallen (Co-NC), bereid m.b.v. colloï dale technieken. Deze aanpak biedt in principe controle over de grootte, vorm en samenstelling van de Co-NC. De navolgende bevestiging van de $\mathrm{Co}-\mathrm{NC}$ aan een dragermateriaal en activering zijn echter even belangrijk voor de toepassing als katalysator, maar hier is nog relatief weinig onderzoek aan gedaan voor onedele metalen. Het doel van het in dit proefschrift beschreven onderzoek is het bestuderen van de bereiding van modelkatalysatoren op basis van Co-NC en hun toepassing in FT om de relatie tussen kobaltstructuur en katalytische prestaties te onderzoeken.

In Hoofdstuk 2 hebben we het effect van oxidatieve behandelingen van $\mathrm{Co}-\mathrm{NC}$ op hun bevestiging aan koolstofnanobuisjes (CNT) en activering voor FT onderzocht. Bevestiging van $\varepsilon$-kobaltnanokristallen resulteerde in clustering van de $\mathrm{NC}$ en lage activiteit. $\mathrm{Na}$ oxidatie van de $\mathrm{NC}$ tot $\mathrm{CoO}$ bij lage temperatuur werden de NC uniform bevestigd aan de CNT. De liganden werden gemakkelijk verwijderd tijdens in situ reductie en hoge activiteit werd verkregen. Het was zelfs mogelijk om een op NC gebaseerde kobaltkatalysator volledig te activeren voor FT met behulp van deze milde procedure. Oxidatie tot $\mathrm{Co}_{3} \mathrm{O}_{4}$ bij hoge temperatuur leidde tot meer deeltjesgroei tijdens in situ reductie of FT en dus was de katalytische activiteit lager. Een zware oxidatieve behandeling was dus onnodig en zelf contraproductief. Milde oxidatie bij lage temperatuur genoot derhalve de voorkeur als behandeling voor de bevestiging en activering van de katalysatoren op basis van Co-NC.

In Hoofdstuk 3 hebben we de impact van oxidatie van de drager bestaande uit CNT op de structuur en prestaties van kobaltkatalysatoren in FT onderzocht. Goed gedefinieerde modelkatalysatoren op CNT en geoxideerde CNT (oxCNT) met 10 gew. \% kobaltbelading werden gesynthetiseerd door bevestiging van $6 \mathrm{~nm}$ colloï dale $\mathrm{CoO}-\mathrm{NC}$. De CoO-NC op onbehandelde CNT werden volledig gereduceerd tot $\mathrm{Co}-\mathrm{NC}$ en groeiden tot $11 \mathrm{~nm}$ tijdens reductie. Dit in tegenstelling tot oxCNT, waar slechts $\sim 50 \%$ CoO-reductie werd waargenomen en de NC $7 \mathrm{~nm}$ bleven, wat duidde op een sterkere metaal-dragerinteractie met oxCNT. Co-NC/CNT waren uitzonderlijk actief in FT met een omzettingsfrequentie 
(TOF) van $150 \cdot 10^{-3} \mathrm{~s}^{-1}$ en relatief stabiel. Ter vergelijking, de TOF op oxCNT was slechts $63 \cdot 10^{-3} \mathrm{~s}^{-1}$ en de katalysator deactiveerde sterker. Met behulp van in situ XRD bleek oxidatie van CNT te resulteren in $32 \%$ minder kristallijn, metallisch kobalt, wat samenviel met een $35 \%$ lagere initiële TOF. De sterkere deactivering van Co-NC op geoxideerde CNT kon niet worden verklaard door deeltjesgroei, herstructurering van de $\mathrm{NC}$, of oxidatie van Co tijdens FT, waardoor een verhoogde afzetting van koolstofhoudende verbindingen als een mogelijke oorzaak overbleef.

In Hoofdstuk 4 hebben we de bereiding van $\mathrm{Co}-\mathrm{NC}$ op $\mathrm{TiO}_{2}$ en $\mathrm{SiO}_{2}$ als dragermateriaal onderzocht en hun katalytische prestaties in FT bestudeerd. Co-NC van 3-12 nm werden gesynthetiseerd met een hete-injectiemethode, geoxideerd bij lage temperatuur om magnetische interacties te beperken en vervolgens bevestigd aan $\mathrm{TiO}_{2}$ of $\mathrm{SiO}_{2}$. De katalytische activiteit van de meeste $\mathrm{TiO}_{2}$-gedragen monsters kwam overeen met die van een $\mathrm{Co} / \mathrm{TiO}_{2}$ monster bereid d.m.v. impregnatie van kobaltnitraat, waaruit bleek dat relevante data verkregen werd met deze modelkatalysatoren. De op kobaltgewicht gebaseerde activiteit van $3 \mathrm{~nm}$ Co- $\mathrm{NC}$ op $\mathrm{TiO}_{2}$ was vijfmaal lager dan verwacht en dit werd gedeeltelijk toegeschreven aan verlies van kobalt aan de drager. Verder groeiden de kobaltdeeltjes op $\mathrm{TiO}_{2}$ en de $3 \mathrm{~nm}$ Co-NC op $\mathrm{SiO}_{2}$ tijdens reductie en FT, terwijl 6 en $9 \mathrm{~nm}$ Co-NC op $\mathrm{SiO}_{2}$ stabiel waren. We stelden voor dat de interactie tussen $\mathrm{Co}(\mathrm{O})$ en $\mathrm{TiO}_{2}$ verantwoordelijk was voor de verminderde stabiliteit van de Co-NC.

In Hoofdstuk 5 hebben we de synthese van schijfvormige Co-NC, hun bevestiging aan een drager en het effect van hun anisotropie op FT onderzocht. Schijfvormige Co-NC met een hexagonale dichtste pakking (hdp) werden gelijktijdig gesynthetiseerd met bolvormige $\mathrm{NC}$ met de $\varepsilon$-kobaltstructuur. $\mathrm{Na}$ oxidatie tot $\mathrm{CoO}$ werden deze $\mathrm{NC}$ afgezet op $\mathrm{SiO}_{2}$ met een laag specifiek oppervlak. Elektronenmicroscopische analyse toonde aan dat numeriek $66 \%$ van de afgezette CoO-NC schijven en $34 \%$ bollen waren. Na in situ reductie van de $\mathrm{CoO}-\mathrm{NC}$ tot $\mathrm{Co}$ was de FT-activiteit van de katalysator met schijven slechts de helft van die van de katalysator met enkel bolvormige Co-NC. Interessant is dat de katalysatoren stabiel waren de grootte en vorm van de NC zelfs voor de schijfvormige Co-NC behouden bleef. XPS wees uit dat de meeste amineliganden werden verwijderd tijdens activering/FT en in situ XRD onthulde dat de kristalstructuur van beide katalysatoren tijdens FT vergelijkbaar was, nl. polykristallijne Co-NC met kristaldomeinen van 3-5 nm en ongeveer $65 \%$ hdp Co en 21-32\% Co met kubische dichtste pakking. Op basis van deze experimenten stellen we voorlopig voor dat het veranderen van bolvormige naar schijfvormige Co-NC de FTactiviteit verlaagde door een toename van de relatieve hoeveelheid hdp (0001)-facetten.

In Hoofdstuk 6 hebben we een overzicht gepresenteerd van de recente literatuur over het reguleren van metaal-dragerinteractie (MSI), met focus op strategieën om de katalytische prestaties te verhogen. Dit onderwerp heeft de afgelopen jaren veel aandacht gekregen, maar een systematische rationalisatie van het veld ontbreekt vanwege de grote diversiteit in katalysatoren, reacties en MSI-modificatiesstrategieën. In dit hoofdstuk hebben we de recente vooruitgang in MSI-modificatiesstrategieën om de katalytische prestaties voor verschillende reacties te verhogen behandeld en gecategoriseerd. Verder hebben we recent gerapporteerde productiviteitsverhogingen in $\mathrm{C}_{1}$-chemie als gevolg van MSI-regulatie gekwantificeerd. Onze analyse wees uit dat tot vijftienmaal 
productiviteitsverhoging is bereikt en dat MSI een grote impact heeft voor metaalnanodeeltjes kleiner dan $4 \mathrm{~nm}$.

In Hoofdstuk 7 hebben we reductie-oxidatie-reductie (ROR) behandelingen onderzocht als methode om de metaal-dragerinteractie in gedragen kobaltkatalysatoren te reguleren om zo hun FT-prestaties te verhogen. Reductie van kobaltoxide op reduceerbare $\left(\mathrm{TiO}_{2}\right.$ en $\left.\mathrm{Nb}_{2} \mathrm{O}_{5}\right)$ en onreduceerbare $\left(\alpha-\mathrm{Al}_{2} \mathrm{O}_{3}\right)$ metaaloxiden gevolgd door gecontroleerde oxidatie leidde tot holle kobaltoxidedeeltjes via het Kirkendall-effect. Een tweede reductie resulteerde in een verdubbeling van de $\mathrm{H}_{2}$-opname voor de $\mathrm{TiO}_{2}$ - en $\mathrm{Nb}_{2} \mathrm{O}_{5}$-gedragen monsters en een afname voor het $\alpha-\mathrm{Al}_{2} \mathrm{O}_{3}$-gedragen monster. Dit effect werd toegeschreven aan modulatie van de metaal-dragerinteractie in plaats van herverdeling van het kobalt. De gemodificeerde metaal-dragerinteractie verdubbelde de katalytische FT-activiteit van Co op $\mathrm{TiO}_{2}$ en $\mathrm{Nb}_{2} \mathrm{O}_{5}$, terwijl de activiteit van $\mathrm{Co}$ op $\alpha-\mathrm{Al}_{2} \mathrm{O}_{3}$ licht afnam. De toename in activiteit werd veroorzaakt door meer toegankelijke kobaltoppervlaktes zoals bleek uit de constante TOF, terwijl het volledige promotie-effect van de $\mathrm{TiO}_{\mathrm{x}^{-}}$of $\mathrm{NbO}_{\mathrm{x}}$-verbindingen behouden bleef. Het efficiëntere gebruik van het kobalt- en dragergrensvlak leidde tot uitzonderlijk actieve FT-katalysatoren.

\section{Afsluitende opmerkingen}

Het eerste doel was een methode te ontwikkelen voor het bereiden van gedragen modelkobaltkatalysatoren die relevant zijn voor de Fischer-Tropsch synthese. Oxidatie van gesynthetiseerde $\mathrm{Co}-\mathrm{NC}$ bij kamertemperatuur werd geï dentificeerd als meest geschikte behandeling voor een uniforme verdeling van de Co-NC over een drager en efficiënte activering, terwijl de impact op de eigenschappen van de Co-NC geminimaliseerd werd. In de hoofdstukken 2 tot en met 5 hebben we de veelzijdigheid van deze aanpak gedemonstreerd, aangezien deze werd toegepast om Co-NC op CNT, zowel onbehandeld als geoxideerd, en op de oxidische dragers, $\mathrm{TiO}_{2}$ en $\mathrm{SiO}_{2}$, te bereiden. Het grootste nadeel van deze benadering voor de synthese van modelkatalysatoren is het onvermijdelijke verlies van controle over de kristalstructuur van het metaal als gevolg van oxidatie. We verwachten dat deze strategie van opzettelijke oxidatie van gesynthetiseerde nanokristallen (NC) bij lage temperatuur om magnetische interacties en de daaruit volgende clustering van NC te omzeilen nuttig zou kunnen zijn om ook NC van andere ferromagnetische metalen uniform te bevestigen aan dragers.

Momenteel zijn deze op NC-gebaseerde katalysatoren vooral geschikt voor modelstudies en niet voor industriële toepassing vanwege hun typisch delicate en dure syntheseprocedure. Zeer weinig op NC-gebaseerde katalysatoren zijn daarom commercieel verkrijgbaar. De relevantie voor de industrie zit hem echter in de inzichten die mogelijk kunnen worden verkregen uit de modelstudies die nu kunnen worden uitgevoerd. Verschillende voorbeelden van dergelijke studies zijn gepresenteerd in dit proefschrift, waarin de gesynthetiseerde op NC-gebaseerde katalysatoren worden gebruikt om structuur-prestatie-relaties vast te stellen.

Ten eerste versterkten functionele groepen en defecten op CNT de metaaldragerinteractie en maakten ze de katalysatoren beter bestand tegen deeltjesgroei, maar ze verlaagden tegelijkertijd de intrinsieke katalytische activiteit en selectiviteit door het 
verminderen va de hoeveelheid kristallijn kobalt en het verhogen van de deactiveringssnelheid. Opmerkelijk genoeg was de activiteit van Co-NC op onbehandelde CNT uitzonderlijk hoog met een TOF van $150 \cdot 10^{-3} \mathrm{~s}^{-1}$. Desondanks blijven er vragen onbeantwoord, zoals het type zuurstofbevattende functionele groepen op CNT, de locatie van het amorfe kobalt en de oorzaak van de waargenomen deactivering op oxCNT. Als het begrip van deze metaal-dragerinteracties vordert, kan het hopelijk worden geëxtrapoleerd om optimale grensvlakken te ontwerpen op industrieel relevantere dragers.

Ten tweede waren $\mathrm{Co}-\mathrm{NC}$ op $\mathrm{SiO}_{2}$ beter bestand tegen deeltjesgroei dan op $\mathrm{TiO}_{2}$. De

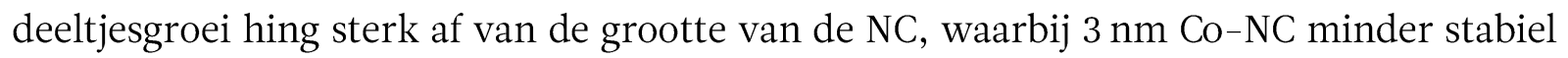
waren dan $6 \mathrm{~nm}$ Co-NC en Co-NC van alle grootten op $\mathrm{TiO}_{2}$ uiteindelijk groeiden tot ongeveer $12 \mathrm{~nm}$. De implicatie van deze waarneming is dat kobaltdeeltjes kleiner dan $12 \mathrm{~nm}$ niet stabiel zijn op dit specifieke type $\mathrm{TiO}_{2}$, dus is het de vraag of het de moeite waard is om streven naar nanodeeltjes kleiner dan $12 \mathrm{~nm}$ voor dit type katalysator.

Ten derde werd een effect van NC-vorm op FT-prestaties waargenomen. Lagere FTactiviteit werd verkregen met schijfvormige dan met bolvormige Co-NC en dit werd voorlopig toegeschreven aan verhoogde blootstelling van hdp (0001)-facetten op de schijven. Opmerkelijk is dat de schijfvorm van de Co-NC de FT-omstandigheden langer dan 100 uur heeft doorstaan. Dit betekent dat in principe andere vormen het potentieel hebben om FT-prestaties te verhogen, als tenminste actievere facetten blootgesteld worden.

De geavanceerde studies die uitgevoerd kunnen worden met op NC-gebaseerde katalysatoren zijn niet beperkt tot de voorbeelden die in dit proefschrift zijn beschreven. Andere interessante kobaltstructuur-FT-prestatiestudies kunnen worden uitgevoerd met behulp van NC, zoals het onderzoeken van promotoreffecten door de hoeveelheid en locatie van de promotors nauwkeurig te controleren, of het onderzoeken van katalysatorstabiliteit en groeimechanismen van $\mathrm{NC}$ door de bereiding van een katalysator met een bimodale NC-groottedistributie van gemengde partijen NC. Bovendien kan deze benadering op analoge wijze worden uitgebreid naar andere door kobalt gekatalyseerde reacties of naar NC van verwante metalen.

Naast modelstudies met Co-NC hebben we het samenspel tussen metaalnanodeeltjes en dragers onderzocht. Uit een literatuuronderzoek werd geconcludeerd dat regulering van de metaal-dragerinteractie tot vijftienmaal productiviteitsverhoging kan leiden. Bovendien ontdekten we dat ROR-behandelingen de FT-activiteit van kobalt op reduceerbare dragers verdubbelden door de bedekking door $\mathrm{TiO}_{\mathrm{x}}-$ of $\mathrm{NbO}_{\mathrm{x}}$-verbindingen te verminderen. Dit kan interessant zijn voor de toepassing, omdat het procesintensificatie mogelijk maakt met behulp van een relatief eenvoudige voorbehandeling en het effect stabiel lijkt, in ieder geval gedurende de 100 uur die hier zijn onderzocht.

In het algemeen is het potentieel van $\mathrm{Co}-\mathrm{NC}$ als modelkatalysatoren voor FT onderzocht alsook de regulering van metaal-dragerinteracties voor verbeterde katalytische prestaties. De conclusies van het hier beschreven werk kunnen leiden tot verbeterde katalytische prestaties door rationeel katalysatorontwerp. 
Appendices 


\section{Appendices}

\section{Appendix A}

\section{Electron diffraction of Co- and CoO-NC}

Electron diffraction was performed on NC both untreated and after LT-oxidation. The electron diffraction pattern of the untreated NC was measured under cryo conditions using the TU/e CryoTitan microscope and the LT-oxidized NC under regular conditions using the Tecnai 12 microscope. The assignment of the diffraction pattern to a specific cobalt phase is given in Table A1.

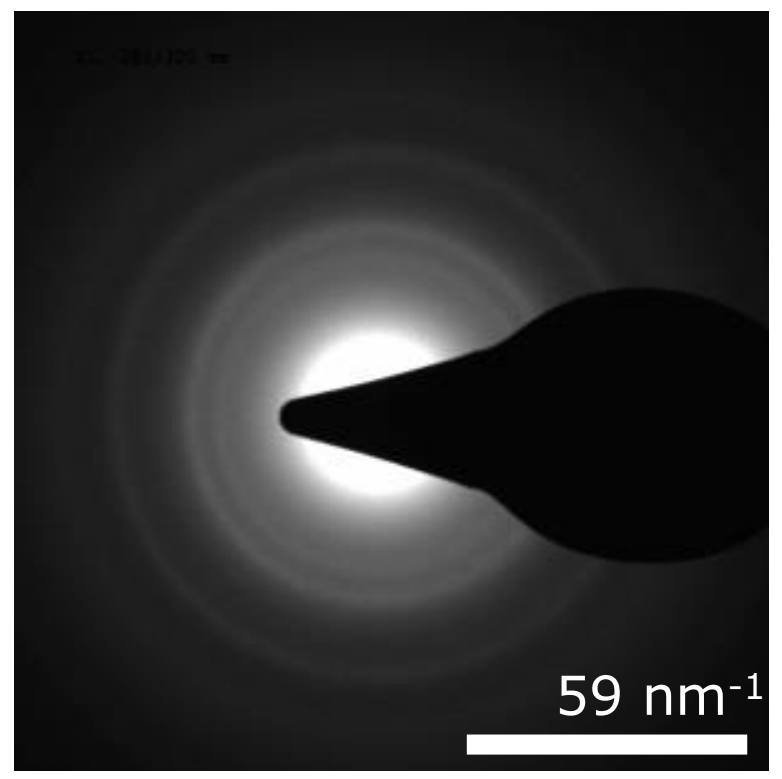

Figure A1. Electron diffraction pattern of $\mathrm{CoO}-\mathrm{NC}$.

Table A1. Experimental d-spacing values for $\mathrm{Co}-$ and $\mathrm{CoO}-\mathrm{NC}$ and reported values for the reference materials. Only the $\mathrm{d}$-spacings with a relative intensity above $10 \%$ are given.

\begin{tabular}{lll|lll}
\hline Co-NC & $\begin{array}{l}\boldsymbol{\varepsilon}-\mathrm{Co} \\
\text { reference }\end{array}$ & $\begin{array}{l}\boldsymbol{\varepsilon}-\mathrm{Co} \\
\text { reference } \\
\text { h } \mathbf{~} \mathbf{l}\end{array}$ & $\begin{array}{l}\mathrm{CoO}-\mathrm{NC} \\
\mathrm{d}(\AA)\end{array}$ & $\begin{array}{l}\mathrm{CoO} \\
\text { reference } \\
\mathrm{d}(\AA)\end{array}$ & $\begin{array}{l}\mathrm{CoO} \\
\text { reference } \\
\text { h } \mathbf{k} \boldsymbol{~}(\AA)\end{array}$ \\
\hline 2.07 & 2.04 & 221 & 2.52 & 2.46 & 111 \\
1.95 & 1.94 & 310 & 2.18 & 2.13 & 200 \\
1.89 & 1.85 & 311 & 1.52 & 1.51 & 220 \\
1.23 & 1.2 & 510 & 1.26 & 1.28 & 311 \\
- & 1.13 & 520 & 1.04 & 1.23 & 222 \\
\hline
\end{tabular}




\section{TEM of Co1/CNT}

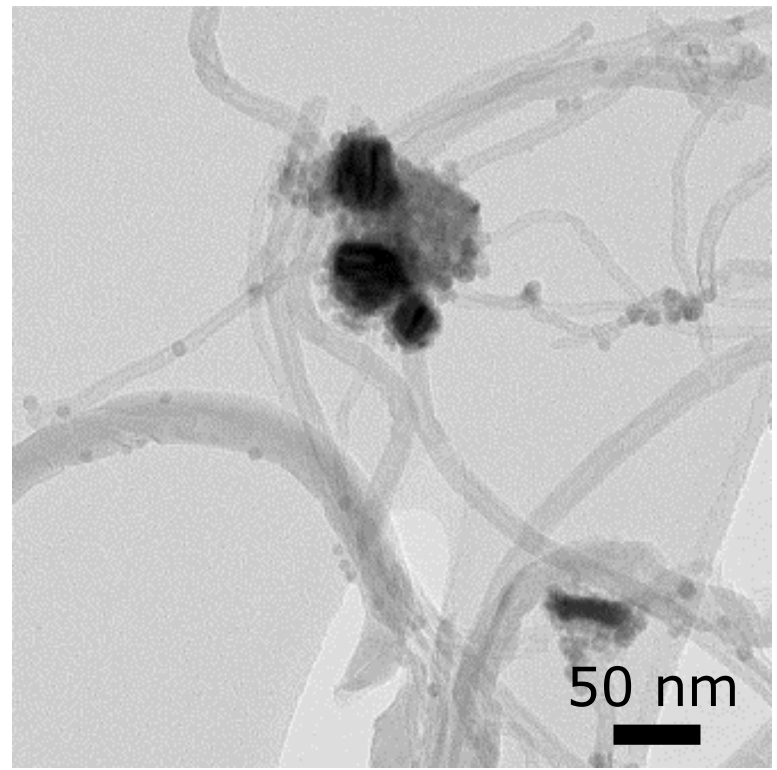

Figure A2. TEM image of Co1/CNT after drying and exposure to air.

\section{XRD of Co/CNT Catalysts}

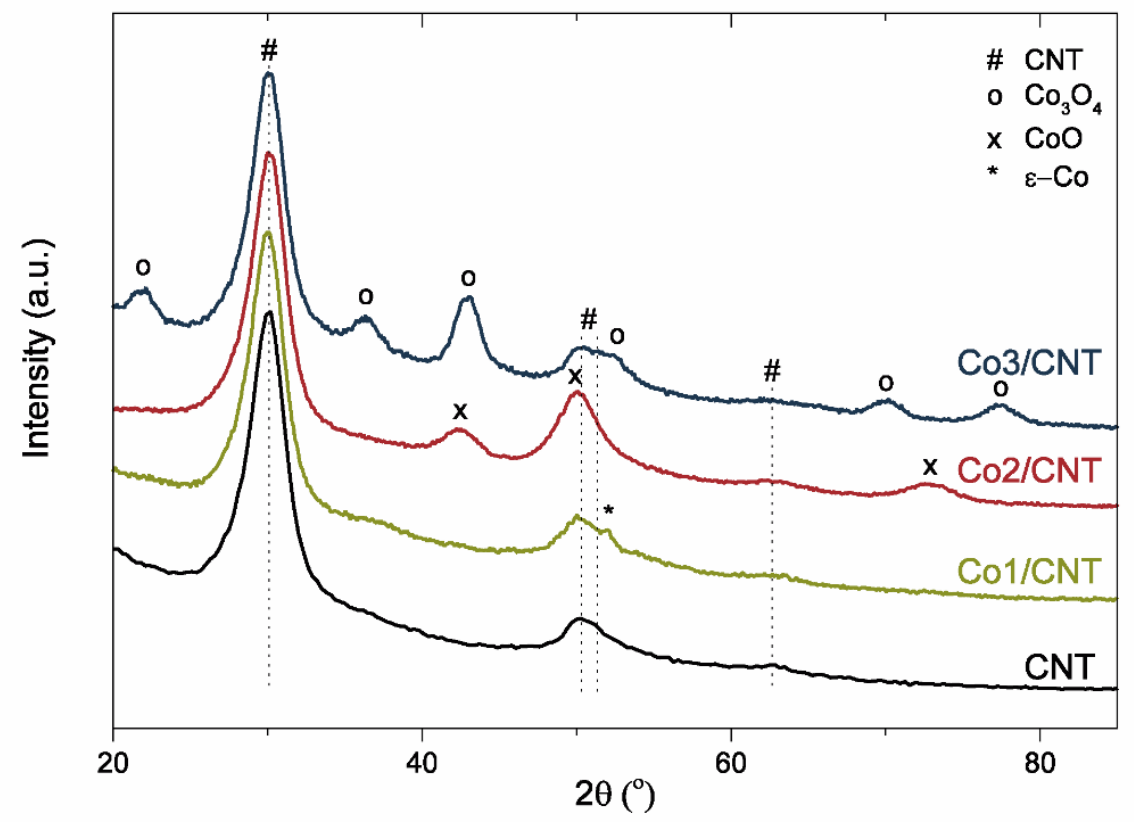

Figure A3. XRD patterns of pristine CNT and the Co/CNT catalysts after various oxidative treatments. 


\section{Particle Size Analysis of Co2/CNT and Co3/CNT}

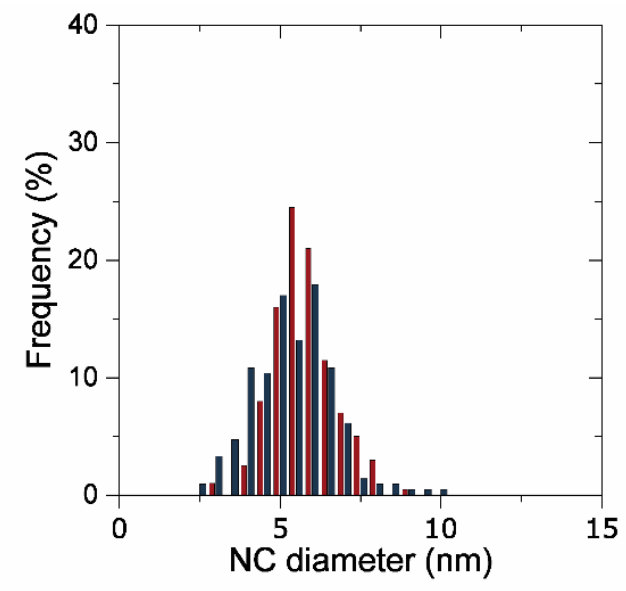

Figure A4. Particle size distributions of Co2/CNT (red) and Co3/CNT (blue).

\section{TGA of $\mathrm{CO} 3 / \mathrm{CNT}$}

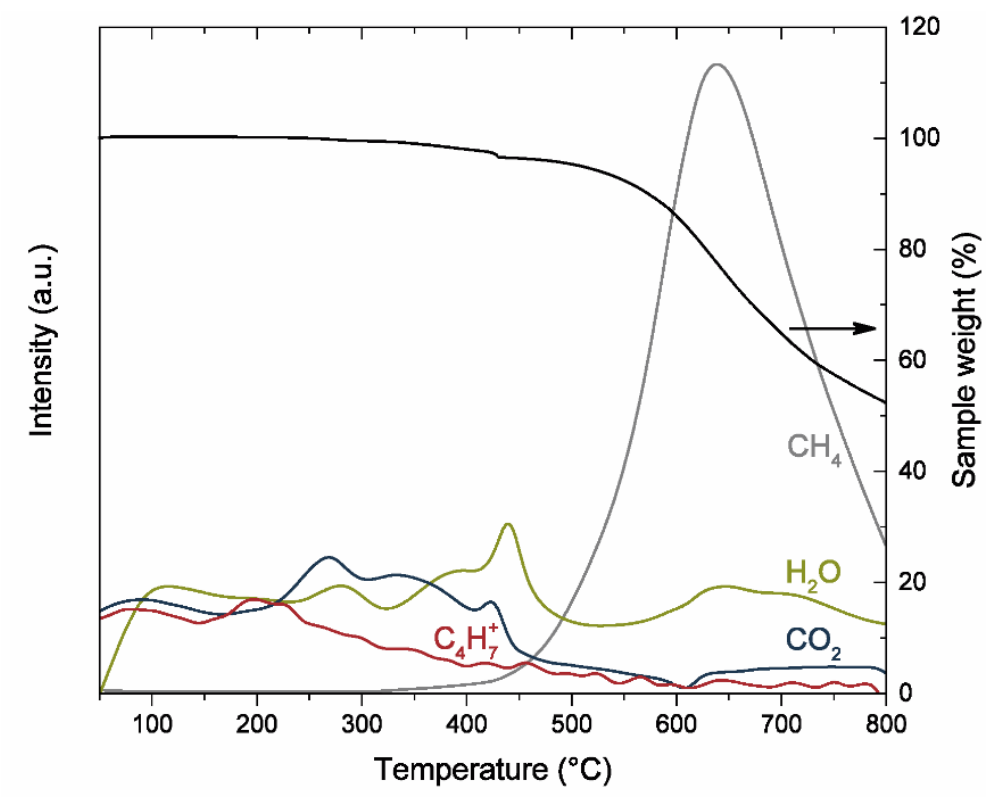

Figure A5. Combined TGA and MS results of $\mathrm{Co} 3 / \mathrm{CNT}$ in $25 \mathrm{vol} \% \mathrm{H}_{2}$ with a heating ramp of $10^{\circ} \mathrm{C} \cdot \mathrm{min}^{-1}$. 


\section{Fischer-Tropsch Synthesis Activity}

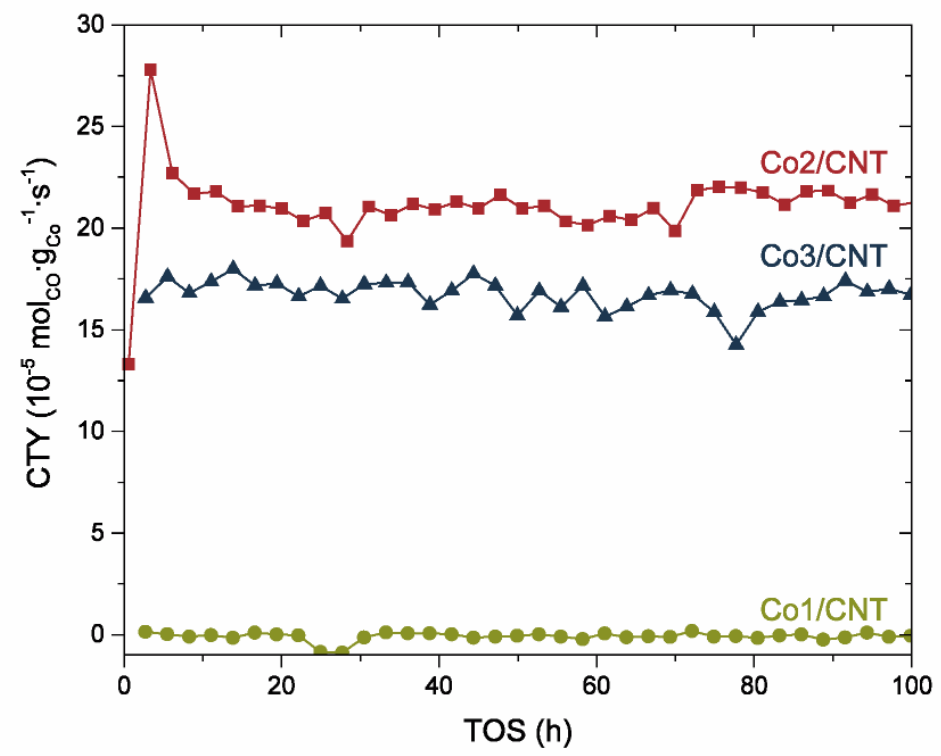

Figure A6. Cobalt time yield during the first $100 \mathrm{~h}$ time-on-stream at $20 \mathrm{bar}, 220^{\circ} \mathrm{C}, \mathrm{H}_{2} / \mathrm{CO}=2 \mathrm{v} / \mathrm{v}$, GHSV $=2350-7850 \mathrm{~h}^{-1}$

\section{Spent Catalysts}

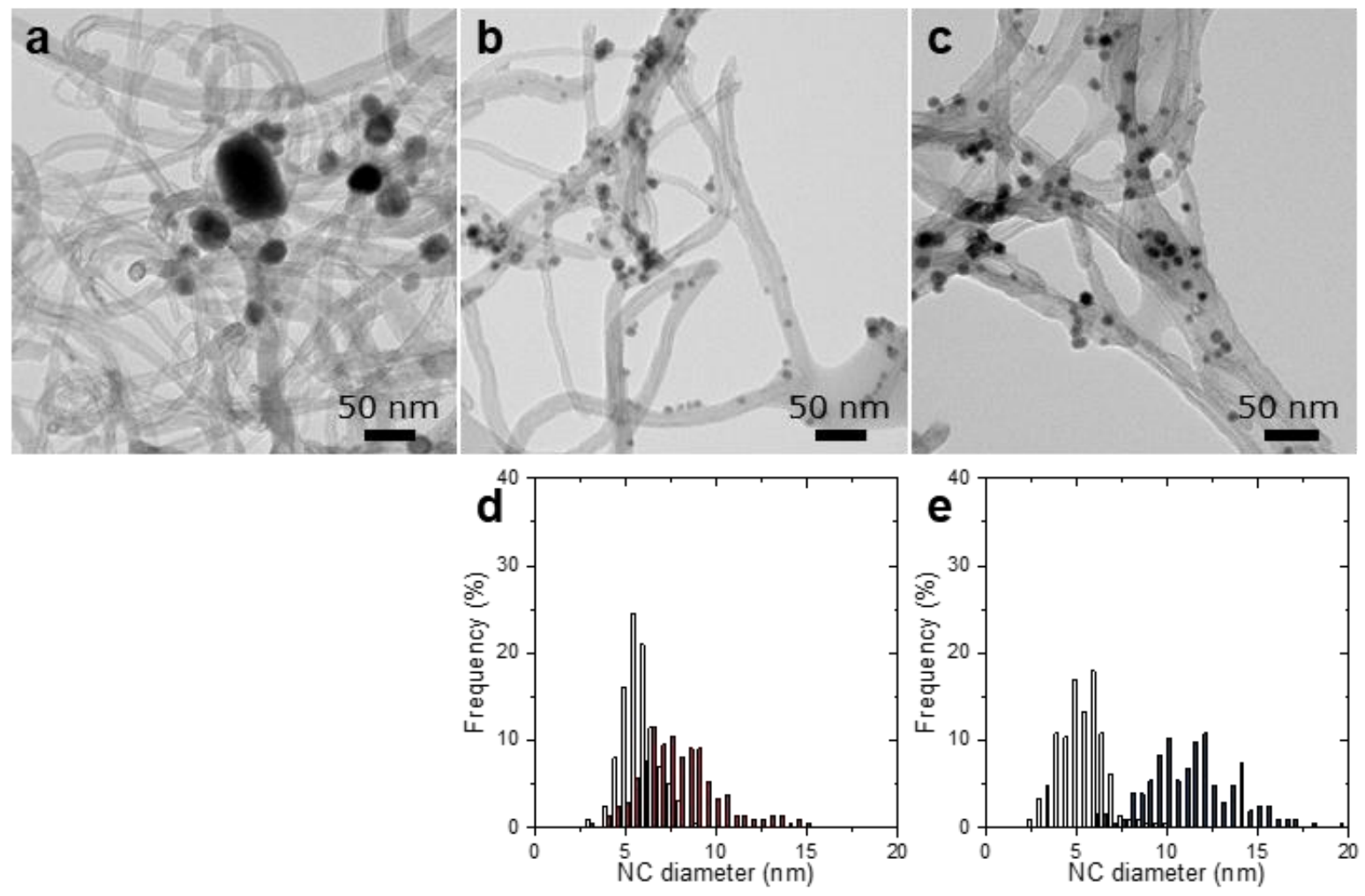

Figure A7. TEM analysis of the spent catalysts. TEM images of the spent catalysts of a Co1/CNT, b Co2/CNT and c Co3/CNT after more than $100 \mathrm{~h}$ TOS and the cobalt particle size distributions of the fresh (open fill) and spent (solid fill) of d Co2/CNT and e Co3/CNT. 


\section{Reported Turnover Frequencies}

The turnover frequencies that were used as a comparison were recalculated from publications by Herranz et al. ${ }^{3}$ and by Delgado et al. ${ }^{4}$ Their catalysts, testing conditions and units to express the TOF differed from ours, which made recalculation necessary. The results of which are given in Table A2.

Herranz et al. synthesized $\mathrm{CoO}-\mathrm{NC}$ using a similar $\mathrm{Co}_{2}(\mathrm{CO})_{8}$ decomposition method as reported here, but in the presence of both oleic acid and trioctylphospine oxide (TOPO) as surfactants. The NC were deposited on silica with a BET surface area of $300 \mathrm{~m}^{2} \cdot \mathrm{g}^{-1}$. The catalytic tests were performed under methanation conditions at 1 bar, $240{ }^{\circ} \mathrm{C}$ and $\mathrm{H}_{2} / \mathrm{CO} / \mathrm{Ar}=3 / 10 / 20 \mathrm{v} / \mathrm{v} / \mathrm{v}$. The TOF values were derived from Figure 5 , in which the TOF was defined as the number of $\mathrm{CO}$ molecules converted per number of Co atoms at the surface per second. According to the unit indicated, the values should be multiplied by a factor $10^{3}$, but the resulting TOF would be unrealistically high and lead to CO conversion levels of $10^{5}-10^{6} \%$. Rather, we assumed the multiplication factor to be $10^{-3}$. The TOF at $220^{\circ} \mathrm{C}$ was derived from the values TOF at $240{ }^{\circ} \mathrm{C}$. To this end, the Arrhenius equation and the experimentally determined activation energy $\left(105 \mathrm{~kJ} \cdot \mathrm{mol}^{-1}\right)$ were used to correct for the temperature difference. Unfortunately, it is not straightforward to correct for the effect of different reaction pressures and feed compositions. Especially at higher pressure, approximately a twofold increase in TOF can be expected. ${ }^{5}$

The NC by Delgado et al. were prepared through reduction of cobalt chloride by sodium borohydride as a reducing agent in the presence of polyvinylpyrrolidone (PVP) as surfactant. Subsequently, the $\mathrm{NC}$ were emplaced on $\mathrm{TiO}_{2} \mathrm{P} 25$. The applied FT conditions were 20 bar, $240{ }^{\circ} \mathrm{C}$ and $\mathrm{H}_{2} / \mathrm{CO}=2 \mathrm{v} / \mathrm{v}$ and the TOF values were reported in mol CO converted per mol Co surface atoms per hour. These values were first divided by $3600 \mathrm{~s} \cdot \mathrm{h}$ ${ }^{1}$ to obtain a TOF per second and afterwards recalculated for a reaction temperature of $220{ }^{\circ} \mathrm{C}$ using again the Arrhenius equation. A typical activation energy of $100 \mathrm{~kJ} \cdot \mathrm{mol}^{-1}$ was assumed.

The significant discrepancy between the TOF reported here and those reported previously for LT- or non-oxidized NC-based catalysts was remarkable, because those catalysts were rather comparable to ours, in particular the discussed $\mathrm{Co} / \mathrm{SiO}_{2}$ catalysts. Although the cause was not established, the most significant difference appeared to be in the choice of surfactants. Only oleic acid was used as a surfactant in our NC synthesis, whereas also TOPO was used by Herranz et al. TOPO reportedly poisons the catalytic activity in $\mathrm{CO}_{2}$ hydrogenation ${ }^{6}$ and therefore, a similar poisoning effect could have occurred as well in FT. In the case of Delgado et al., boron from the reducing agent, $\mathrm{NaBH}_{4}$, remained in the catalysts and probably affected the catalytic performance considerably. 
Table A2. Turnover frequencies reported in literature for LT- or non-oxidized NC-based catalysts. The TOF were converted to the same units and to the same reaction temperatures.

\begin{tabular}{|c|c|c|c|c|c|c|}
\hline \multicolumn{3}{|c|}{ Herranz et al. ${ }^{3}$} & \multicolumn{4}{|c|}{ Delgado et al. ${ }^{4}$} \\
\hline $\begin{array}{l}\text { Average } \\
\text { particle } \\
\text { diameter } \\
(\mathrm{nm})\end{array}$ & $\begin{array}{l}\text { TOF at } \\
240^{\circ} \mathrm{C} \\
\left(10^{-3} \mathrm{~s}^{-1}\right)\end{array}$ & $\begin{array}{l}\text { TOF at } \\
220^{\circ} \mathrm{C} \\
\left(10^{-3} \mathrm{~s}^{-1}\right)\end{array}$ & $\begin{array}{l}\text { Average } \\
\text { particle } \\
\text { diameter } \\
(\mathrm{nm})\end{array}$ & $\begin{array}{l}\text { TOF at } \\
240{ }^{\circ} \mathrm{C} \\
\left(\mathrm{h}^{-1}\right)\end{array}$ & $\begin{array}{l}\text { TOF at } \\
240^{\circ} \mathrm{C} \\
\left(10^{-3} \mathrm{~s}^{-1}\right)\end{array}$ & $\begin{array}{l}\text { TOF at } \\
220^{\circ} \mathrm{C} \\
\left(10^{-3} \mathrm{~s}^{-1}\right)\end{array}$ \\
\hline 3.0 & 0.6 & 0.2 & 1.8 & 19 & 5.1 & 2.0 \\
\hline 4.2 & 0.8 & 0.3 & 2.6 & 13 & 3.5 & 1.4 \\
\hline 4.6 & 1.0 & 0.4 & 5.2 & 6.6 & 1.8 & 0.7 \\
\hline 5.3 & 0.8 & 0.3 & 7.0 & 2.1 & 0.6 & 0.2 \\
\hline 10 & 1.9 & 0.7 & - & - & - & - \\
\hline
\end{tabular}

\section{References}

1. Sun, S. \& Murray, C. B. Synthesis of monodisperse cobalt nanocrystals and their assembly into magnetic superlattices. J. Appl. Phys. 85, 4325-4330 (1999).

2. Petit, C., Wang, Z. L. \& Pileni, M. P. Seven-Nanometer Hexagonal Close Packed Cobalt Nanocrystals for High-Temperature Magnetic Applications through a Novel Annealing Process. J. Phys. Chem. B109, 15309-15316 (2005).

3. Herranz, T., Deng, X., Cabot, A., Guo, J. \& Salmeron, M. Influence of the Cobalt Particle Size in the CO Hydrogenation Reaction Studied by In Situ X-Ray Absorption Spectroscopy. J. Phys. Chem. B 113, 10721-10727 (2009).

4. Delgado, J. A. et al. Fischer-Tropsch synthesis catalysed by small $\mathrm{TiO}_{2}$ supported cobalt nanoparticles prepared by sodium borohydride reduction. Appl. Catal. A Gen. 513, 39-46 (2016).

5. den Breejen, J. P. et al. On the Origin of the Cobalt Particle Size Effects in Fischer-Tropsch Catalysis. J. Am. Chem. Soc. 131, 7197-7203 (2009).

6. Iablokov, V. et al. Size-Controlled Model Co Nanoparticle Catalysts for $\mathrm{CO}_{2}$ Hydrogenation: Synthesis, Characterization, and Catalytic Reactions. Nano Lett. 12, 3091-3096 (2012). 


\section{Appendix B}

\section{Methods}

\section{In situ XRD quantitative phase analysis}

In situ XRD results were analyzed by Rietveld refinement using Bruker TOPAS v5.0 software. $\mathrm{A} \mathrm{LaB}_{6}$ standard reference material (660c) from the National Institute of Standards and Technology was measured and fitted using the fundamental parameters approach to determine the emission profile and instrument details. The $\mathrm{K}$-emission profile of Mo was refined to $\mathrm{K}_{\alpha 1}$ emission of $0.708856 \AA$ and $\mathrm{K}_{\alpha 2}$ emission of $0.712992 \AA$, while simultaneously refining a $4^{\text {th }}$ order Chebyshev polynomial background, tube tails, cylindrical $2 \theta$ correction (Sabine), and sample displacement and tilt. The obtained emission profile and instrument details were applied in the analysis of all samples.

The bare supports, CNT and oxCNT, were measured at ambient conditions to establish the contribution of the support as well as the background. The XRD profile was fitted between 9 and $35^{\circ} 2 \theta$ using a $5^{\text {th }}$ order Chebyshev polynomial background. The CNT and oxCNT structures were experimentally determined by refining a graphite structure (ICDD PDF-4+2016, PDF 04-014-0362) through tuning of the lattice parameters within $\pm 0.06 \AA$, crystallite size (Gaussian) and strain (Lorentzian), and preferential orientations in the [111] and [011] directions using the March-Dollase model. The amorphous phase was modelled using two pseudo-Voigt peaks around 10.3 and $21.5^{\circ} 2 \theta$. Simultaneously, cylindrical $2 \theta$ correction (Sabine) and sample displacement and tilt were refined.

The XRD patterns of the catalysts were fitted using the determined (ox)CNT and amorphous phase contributions in combination with identified cobalt phases obtained from the ICDD PDF-4+2016 database, i.e. hcp Co (PDF 04-001-3273), fcc Co (PDF 04-0012681) and CoO (PDF 04-001-8626). The structures of (ox)CNT and the amorphous phase were fixed, leaving only the possibility for scaling. For the Co phases, the lattice parameters were constrained by $\pm 0.05 \AA$, although for most phases variations of $\pm 0.02 \AA$ were obtained. Size broadening was described using Lorentzian functions, while strain broadening was not included in the analysis, as that did not improve the quality of the fit. For hcp Co, preferred orientations in the [100] and [001] directions were also refined using the March-Dollase model. We tentatively propose that this is because of an hcp-fcc intergrowth phase that needs to be taken into account (see results and discussion in the main text). The average crystallite size was determined as the volume weighted mean column length, derived from the integral peak breadth using a crystallite shape factor $(\mathrm{k})$ of 1 . A $5^{\text {th }}$ order Chebyshev polynomial background, cylindrical $2 \theta$ correction (Sabine) and sample displacement and tilt were refined together with the (ox)CNT contribution and the Co and amorphous phases. 
Appendices

\section{Results}

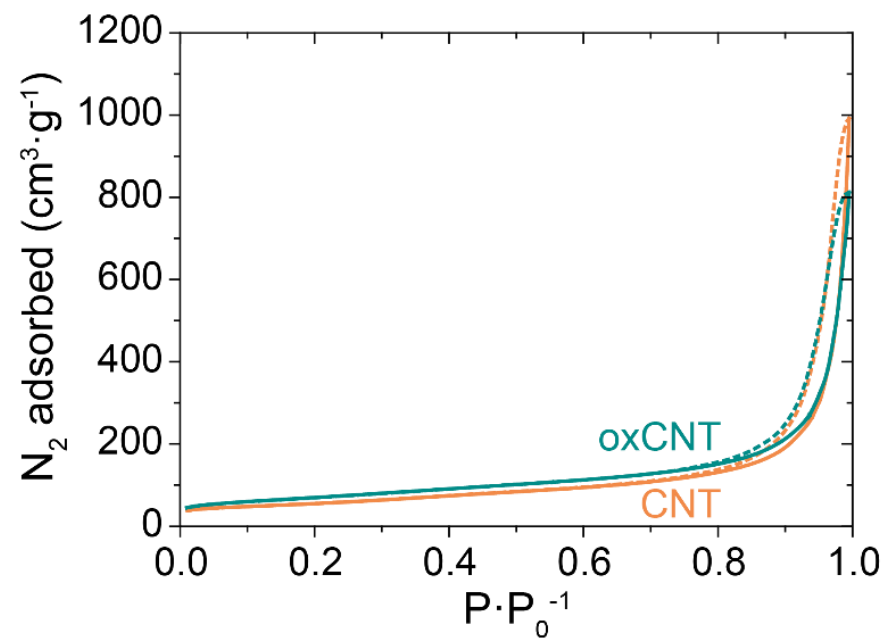

Figure B1. $\mathrm{N}_{2}$-physisorption isotherms of CNT and oxCNT. 

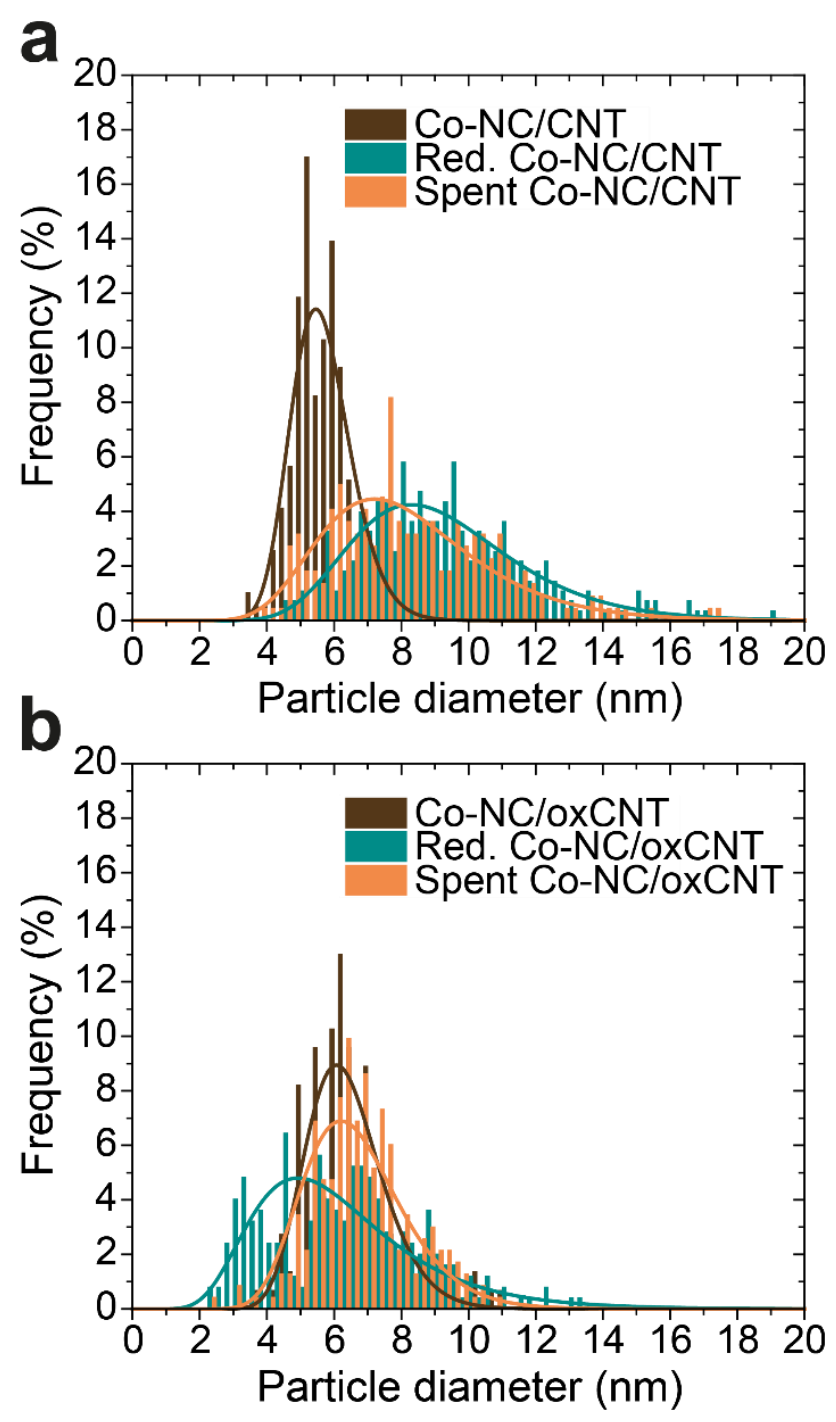

Figure B2. Particle size distributions derived from TEM of the as-prepared samples (brown), the reduced samples (aqua) and spent samples (orange) together with the fitted lognormal distributions. The particle size distributions are given for $\mathbf{a} \mathrm{Co}-\mathrm{NC} / \mathrm{CNT}$ and $\mathbf{b} \mathrm{Co}-\mathrm{NC} /$ oxCNT. Particles $>20 \mathrm{~nm}$ (if present) were not taken into account for the lognormal distributions. 

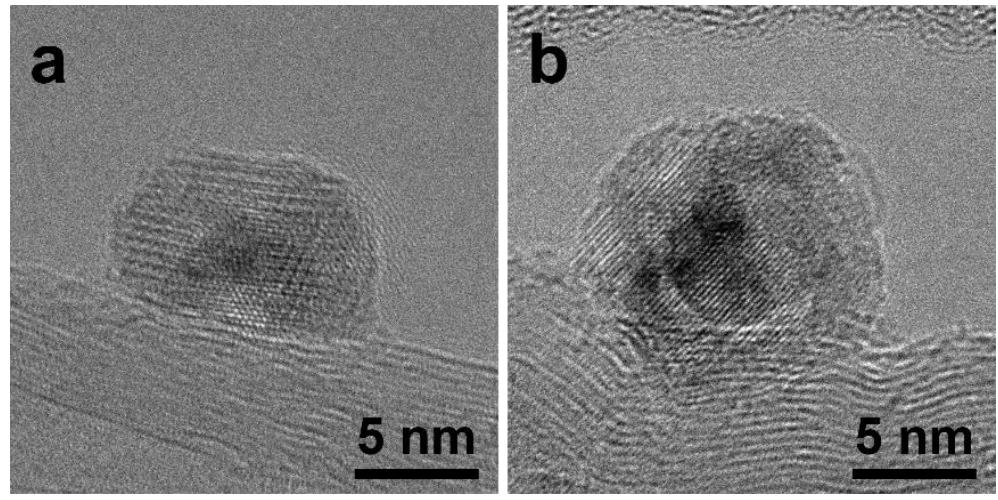

Figure B3. High-resolution TEM results after reduction and passivation of $\mathbf{a} \mathrm{Co}-\mathrm{NC} / \mathrm{CNT}$ and $\mathbf{b} \mathrm{Co}-$ $\mathrm{NC} / \mathrm{oxCNT}$.

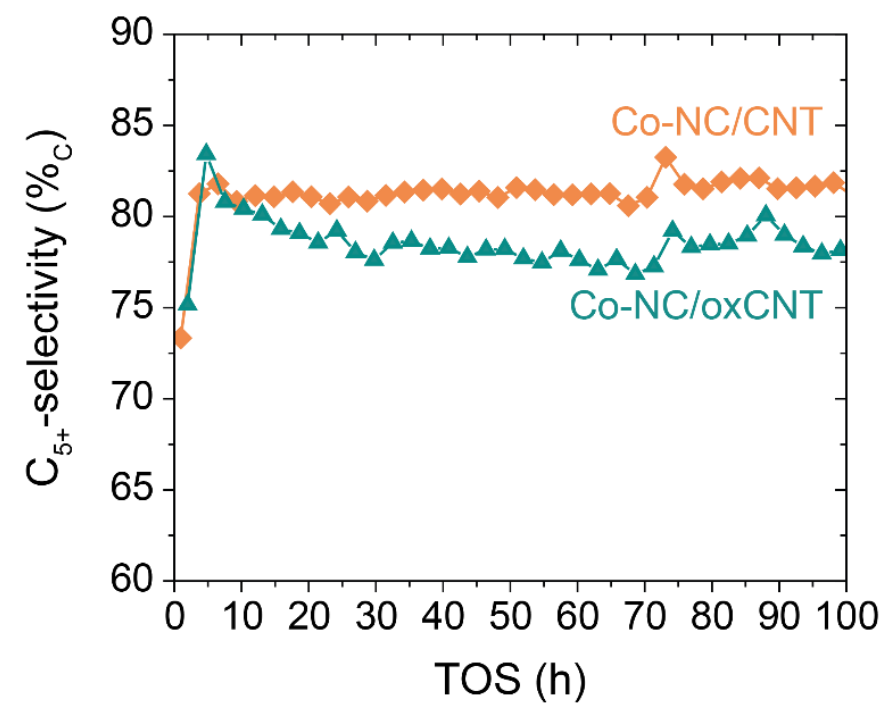

Figure B4. $\mathrm{C}_{5+}-$ selectivity of the two catalysts during $100 \mathrm{~h}$ on stream at $20 \mathrm{bar}, 220{ }^{\circ} \mathrm{C}, \mathrm{H}_{2} / \mathrm{CO}=2$, GHSV $=5950-8900 \mathrm{~h}^{-1}$.

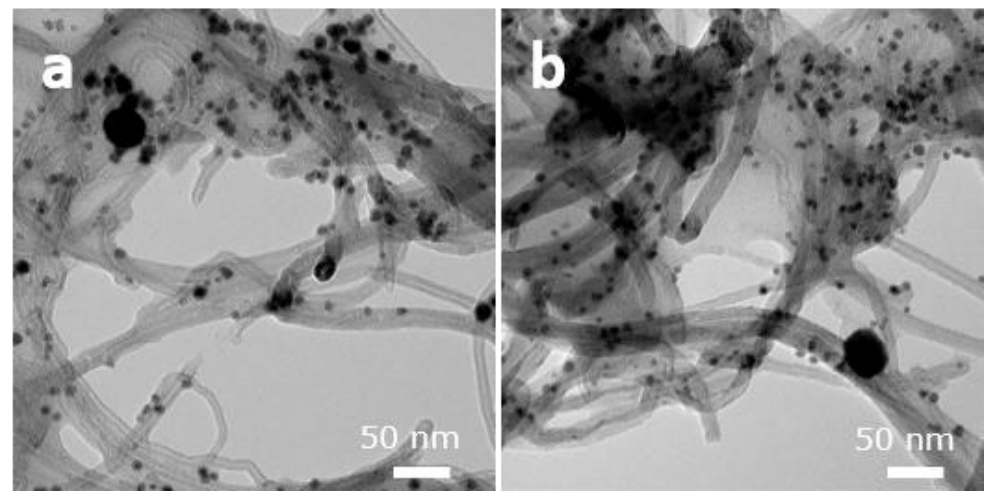

Figure B5. TEM images after $>100 \mathrm{~h} \mathrm{FT}$ and passivation of $\mathbf{a} \mathrm{Co}-\mathrm{NC} / \mathrm{CNT}$ and $\mathbf{b} \mathrm{Co}-\mathrm{NC} / \mathrm{oxCNT}$. 


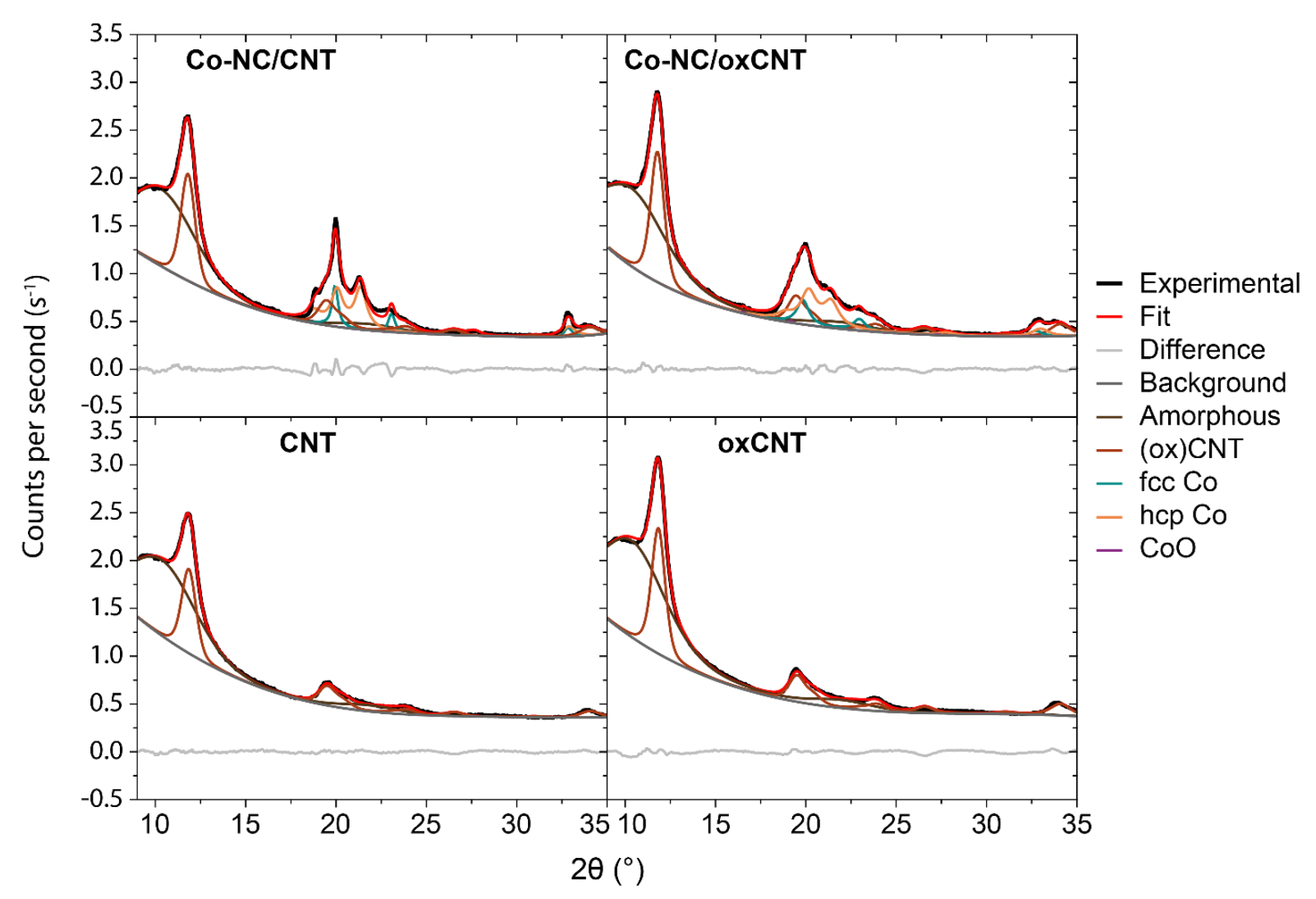

Figure B6. Rietveld Refinement results and diffraction patterns of Co-NC/CNT and Co-NC/oxCNT obtained with in situ XRD during FT between 32 and $42 \mathrm{~h}$ on stream at 10 bar, $220{ }^{\circ} \mathrm{C}$ and $\mathrm{H}_{2} / \mathrm{CO}=2 \mathrm{v} / \mathrm{v}$. Five scans of $2 \mathrm{~h}$ were accumulated and no change in the diffraction patterns was apparent over the course of the reaction. Reference patterns of the bare supports were measured at ambient conditions and refined. 

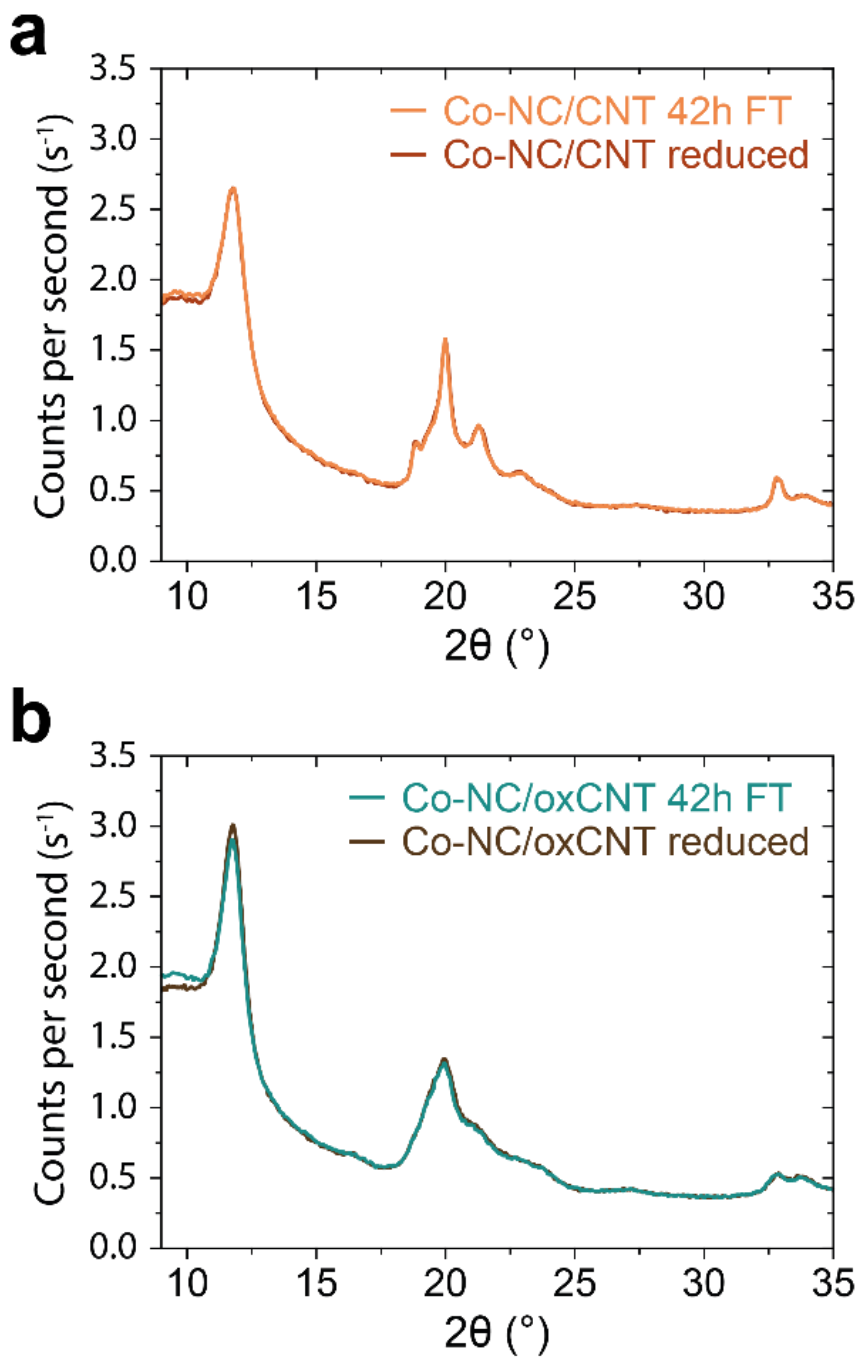

Figure B7. Diffraction patterns of a Co-NC/CNT and b Co-NC/oxCNT obtained with in situ XRD after reduction, measured at $1 \mathrm{bar}, 180^{\circ} \mathrm{C}$ and $25 \mathrm{vol} \% \mathrm{H}_{2}$ in He flow, and during FT between 32 and $42 \mathrm{~h}$ on stream at $10 \mathrm{bar}, 220^{\circ} \mathrm{C}$ and $\mathrm{H}_{2} / \mathrm{CO}=2 \mathrm{v} / \mathrm{v}$. 


\section{Appendix C}

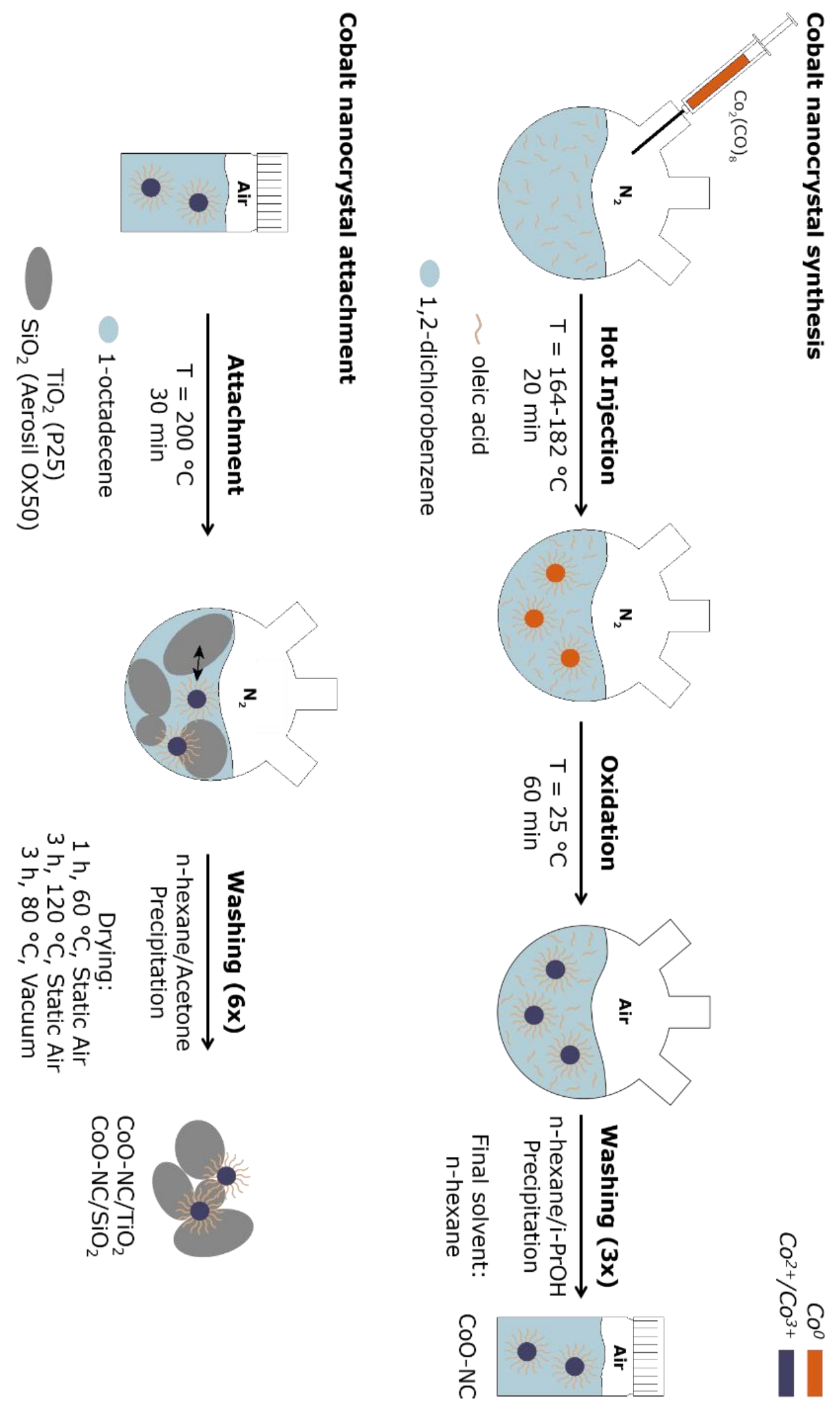

Figure C1. Schematic illustration of the catalyst synthesis procedure. 


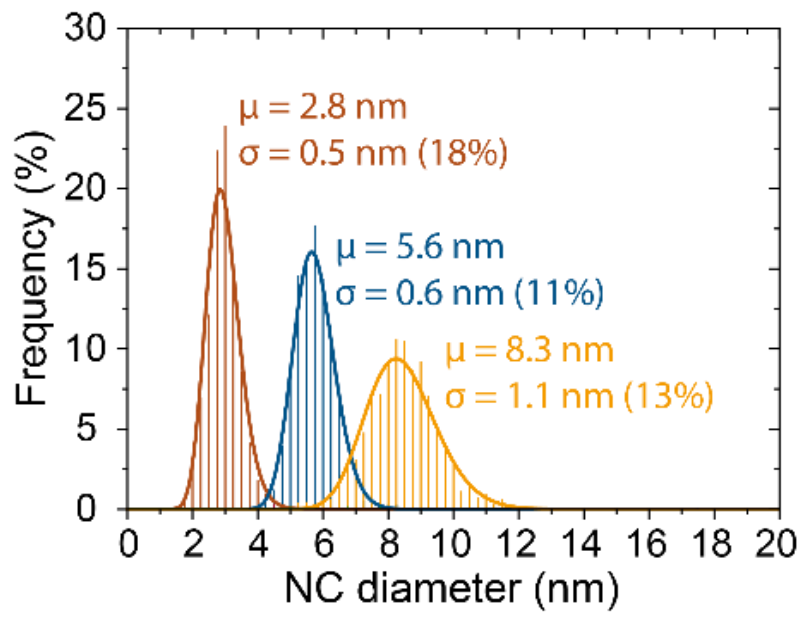

Figure C2. Histograms and lognormal distributions of $\mathrm{Co}-\mathrm{NC}$ that were later attached to $\mathrm{SiO}_{2}$.

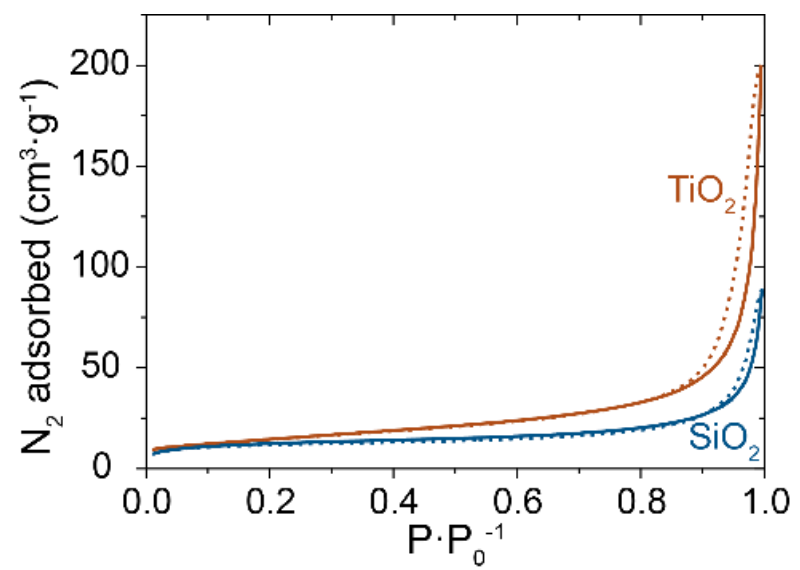

Figure C3. $\mathrm{N}_{2}$-physisorption isotherms of the bare supports, $\mathrm{TiO}_{2}$ and $\mathrm{SiO}_{2}$.

Table C1. $\mathrm{N}_{2}$ - physisorption results of the bare supports, $\mathrm{TiO}_{2}$ and $\mathrm{SiO}_{2}$.

\begin{tabular}{lll}
\hline Support & $\begin{array}{l}\mathrm{SA}_{\mathrm{BET}} \\
\left(\mathrm{m}^{2} \cdot \mathrm{g}^{-1}\right)\end{array}$ & $\begin{array}{l}\text { Pore volume } \\
\left(\mathrm{mL} \cdot \mathrm{g}^{-1}\right)^{\mathrm{a}}\end{array}$ \\
\hline $\mathrm{TiO}_{2} \mathrm{P} 25$ & 53 & 0.31 \\
$\mathrm{SiO}_{2}$ Aerosil OX50 & 43 & 0.14 \\
\hline
\end{tabular}

${ }^{a}$ Determined at $\mathrm{P} / \mathrm{P}_{0}=0.995$ 

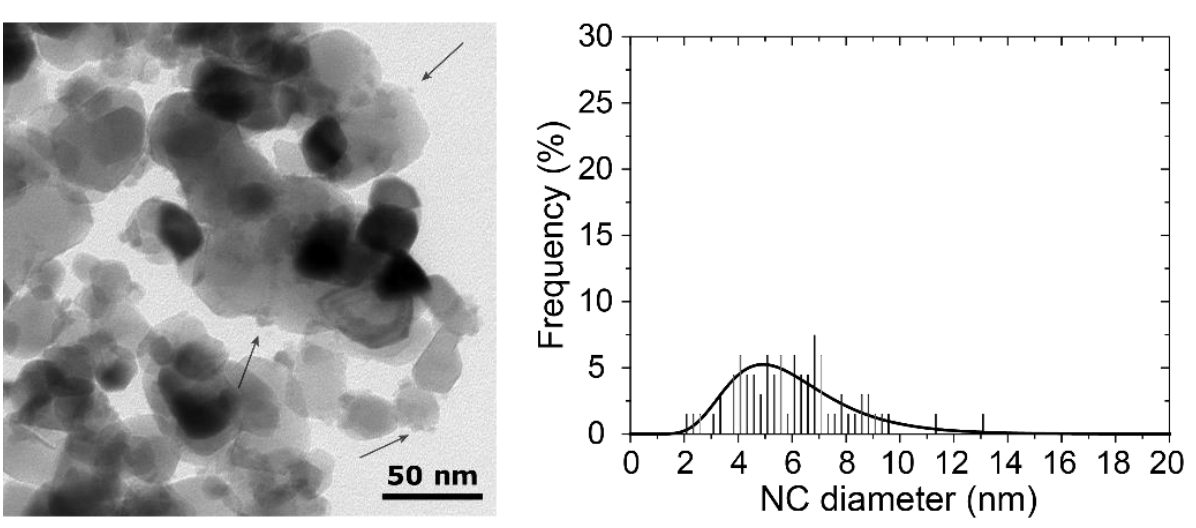

Figure C4. TEM analysis of pristine IWI-Co/ $\mathrm{TiO}_{2}$ after heat treatment in $\mathrm{N}_{2}$. TEM image (left) with the arrows indicating several $\mathrm{Co}_{3} \mathrm{O}_{4}$ particles and the corresponding histogram and lognormal distribution (right). 

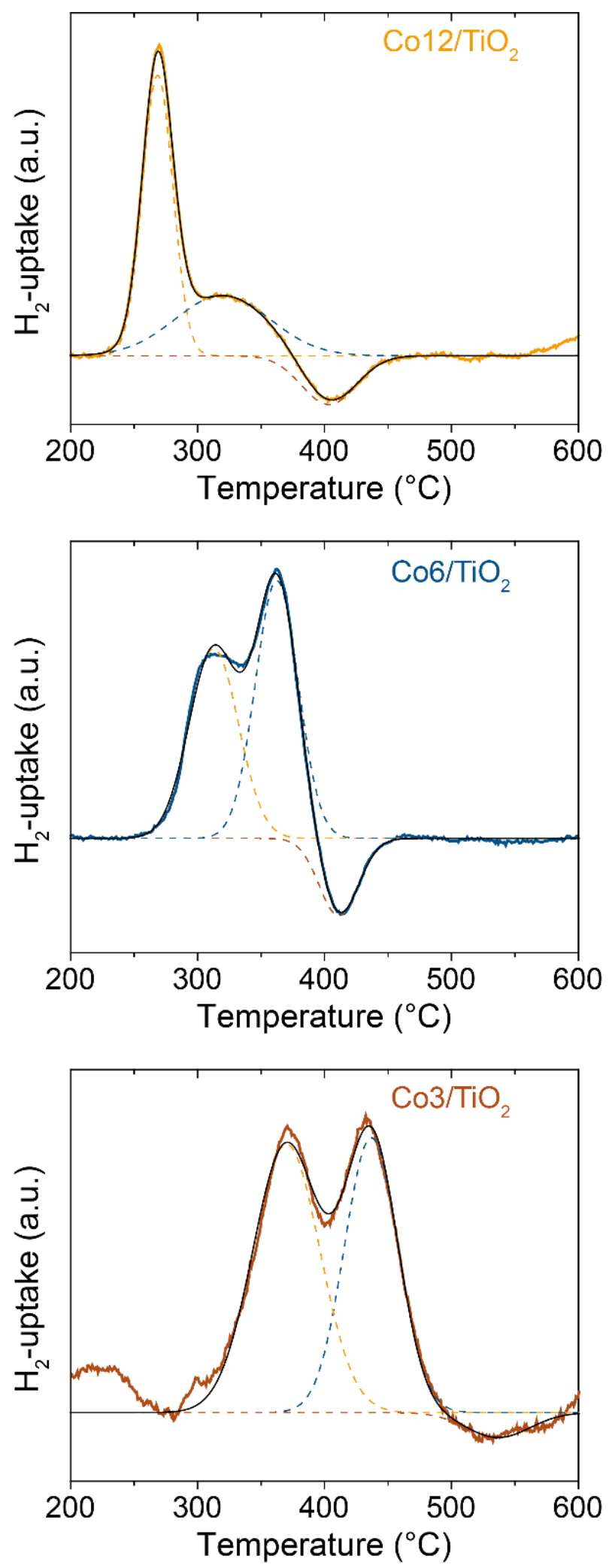

Figure C5. Deconvolution of the TPR profiles of the $\mathrm{Co} / \mathrm{TiO}_{2}$ samples. Three Gaussian contributions (dashed colored lines) were combined to give the overall fit (black solid line) to the experimental data (solid colored lines). 
Appendix C

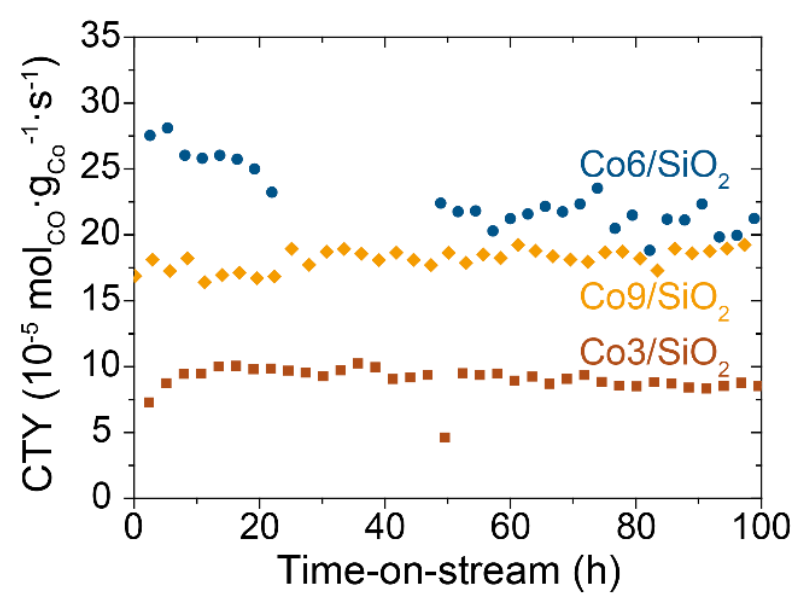

Figure C6. Cobalt-weight-based activity of the $\mathrm{SiO}_{2}$-supported $\mathrm{Co}-\mathrm{NC}$ against time-on-stream. FT was performed at $20 \mathrm{bar}, 220^{\circ} \mathrm{C}, 2 \mathrm{H}_{2} / \mathrm{CO}(\mathrm{v} / \mathrm{v})$, GHSV $=1500-10750 \mathrm{~h}^{-1}$ and $\mathrm{X}_{\mathrm{CO}}=15-30 \%$. 

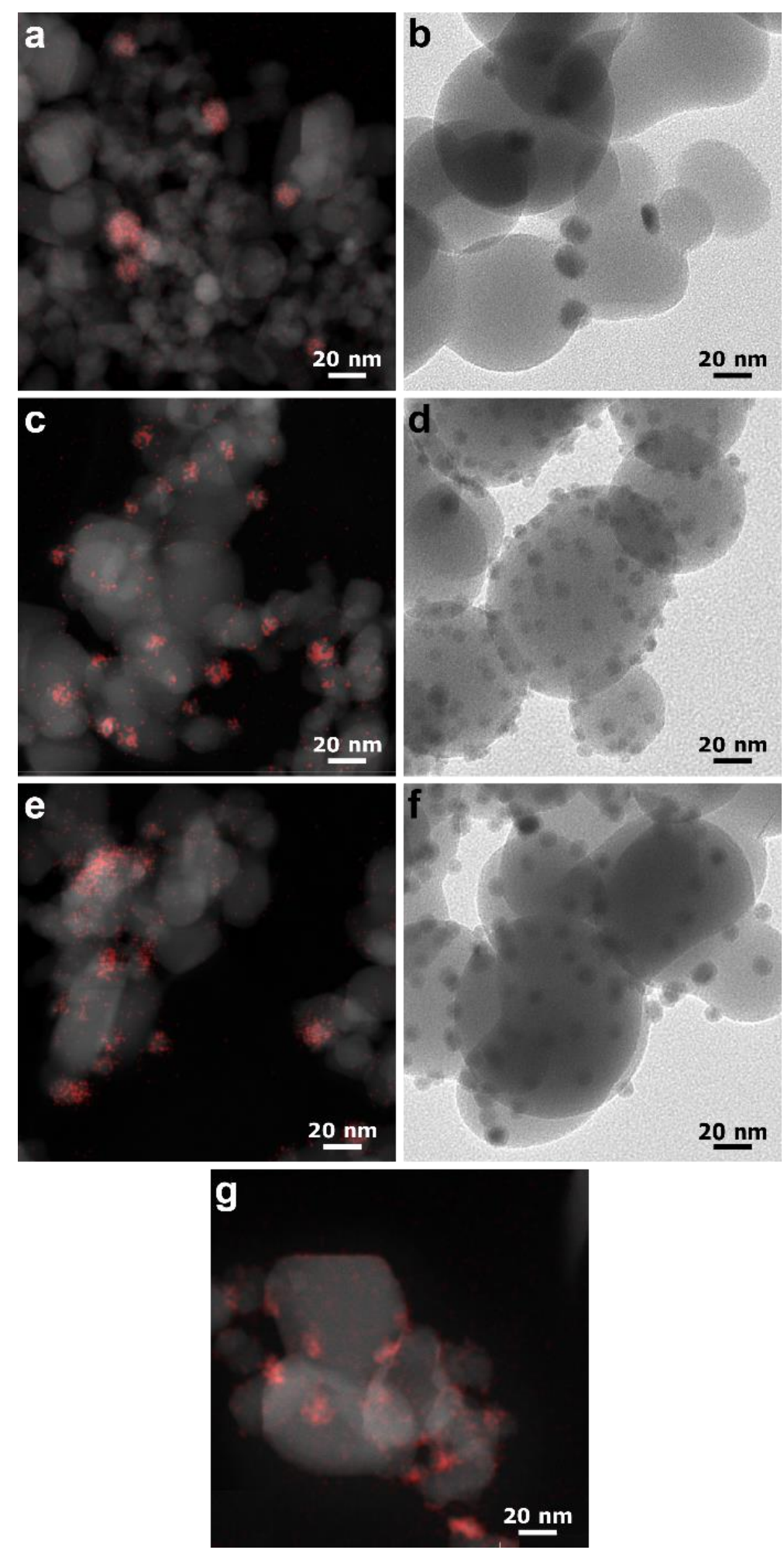

Figure C7. Overview of the samples after catalysis. HAADF-STEM-EDX of spent $\mathrm{Co} / \mathrm{TiO}_{2}$ samples are in the left column (Co in red) and TEM of the $\mathrm{SiO}_{2}$-supported samples is in the right column. a $\mathrm{Co} 3 / \mathrm{TiO}_{2}, \mathbf{b} \mathrm{Co} 3 / \mathrm{SiO}_{2}, \mathbf{c} \mathrm{Co} 6 / \mathrm{TiO}_{2}, \mathbf{d ~ C o 6} / \mathrm{SiO}_{2}, \mathrm{e} \mathrm{Co12} / \mathrm{TiO}_{2}, \mathrm{f} \mathrm{Co} / \mathrm{SiO}_{2}$ and $\mathrm{g} \mathrm{IWI}-\mathrm{Co} / \mathrm{TiO}_{2}$. 

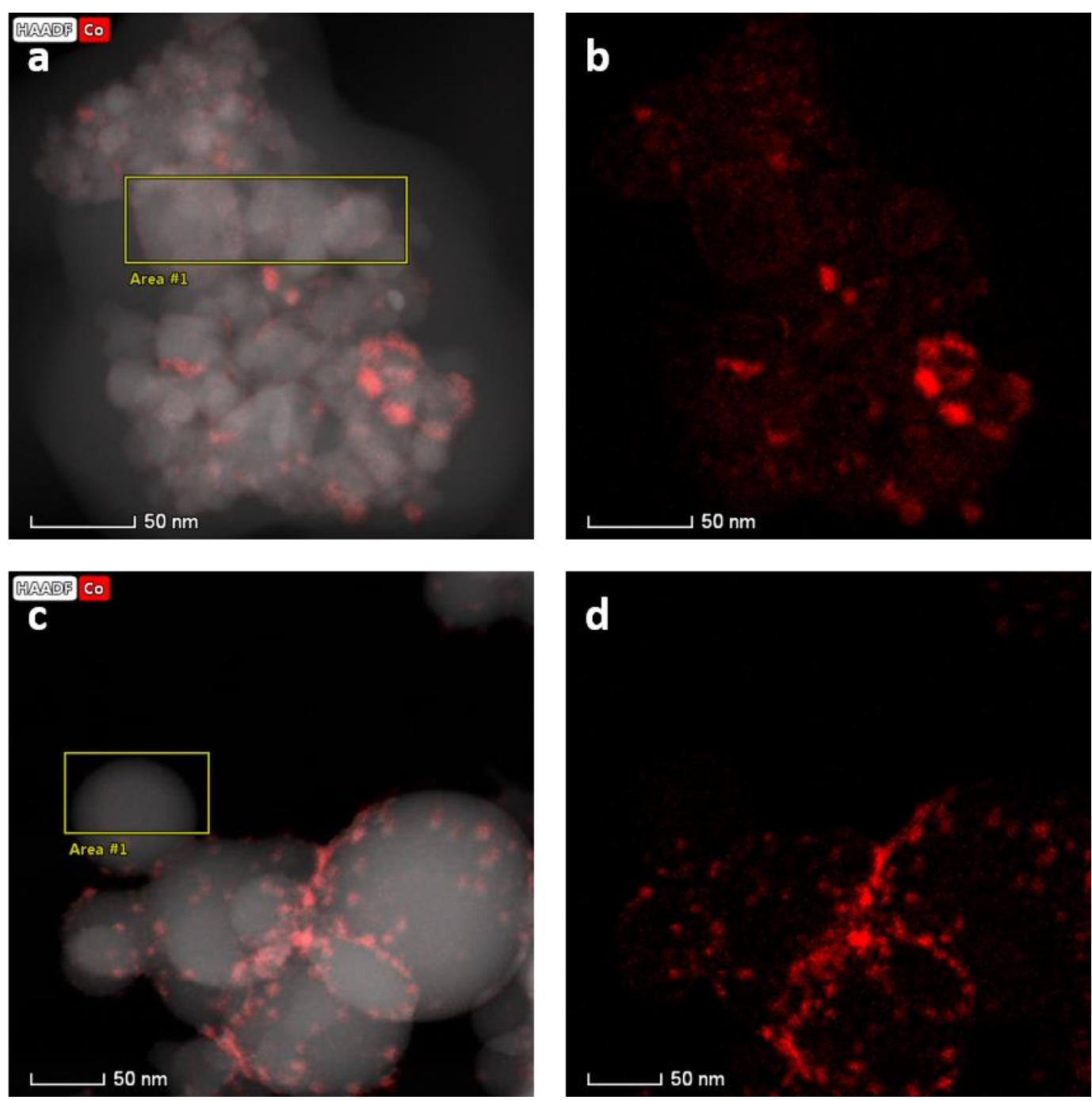

Figure C8. Quantification of the cobalt content in the support of the reduced $\mathrm{Co} / \mathrm{TiO}_{2}$ samples. HAADF-STEM-EDX map of a $\mathrm{Co} 3 / \mathrm{TiO}_{2}$ and $\mathbf{c} \mathrm{Co} 3 / \mathrm{SiO}_{2}$ both after reduction (Co in red) and $\mathbf{b}, \mathbf{d}$ the same location with only the Co signal from EDX, respectively. Parts of the samples without any visible particles are highlighted as Area \#1.

The cobalt content in the support was determined using the ratio between the $\mathrm{Co}, \mathrm{O}$ and $\mathrm{Ti} / \mathrm{Si}$ signals in the highlighted area obtained by quantification of the EDX spectra using FEI Velox software. The same was done for the complete field of view and gave the total Co content, so both in the support and in the NC. The difference between the two represented the amount of Co residing in the NC. The results of multiple EDX maps (4-13) was averaged. 


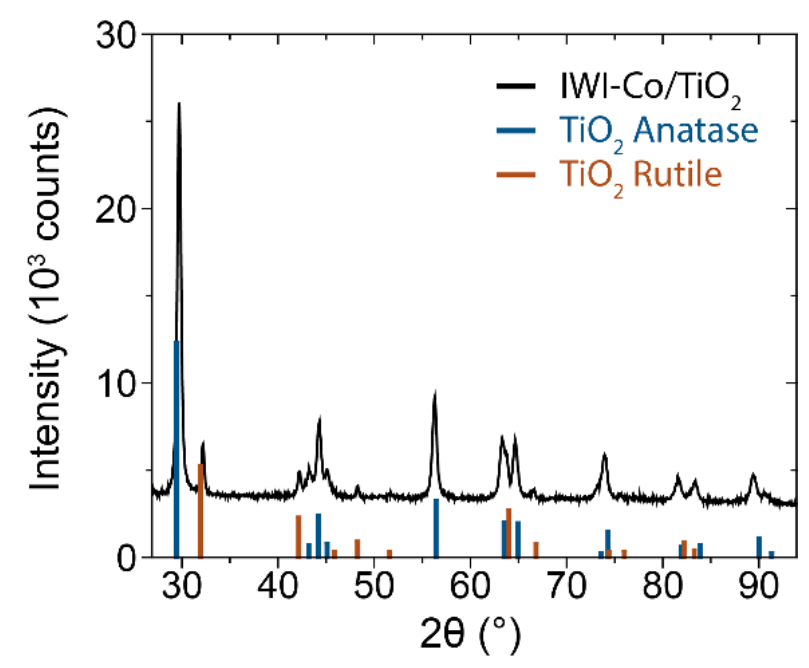

Figure C9. Diffractogram of reduced and passivated IWI- $\mathrm{Co} / \mathrm{TiO}_{2}$ as measured by XRD. The expected diffractions for $\mathrm{TiO}_{2}$ in the anatase and rutile phase are provided. No cobalt phases were apparent. 



Figure C10. Thermodynamic equilibrium composition calculations on the formation of mixed metal-support compounds of $\mathrm{Co} / \mathrm{TiO}_{2}$ (left) and $\mathrm{Co} / \mathrm{SiO}_{2}$ (right). Equilibrium calculations based on the Gibbs free energy were performed with HSC Chemistry 7.1 software using $1 \mathrm{kmol} \mathrm{CoO}(\mathrm{s})$, $100 \mathrm{kmol} \mathrm{TiO}_{2}(\mathrm{~s})$ or $\mathrm{SiO}_{2}(\mathrm{~s})$ and $100 \mathrm{kmol} \mathrm{H}_{2}(\mathrm{~g})$ as starting compounds and a constant number of atoms and pressure ( 1 bar). All available solid $\mathrm{Co}, \mathrm{CoO}_{\mathrm{x}}, \mathrm{Ti}, \mathrm{TiO}_{\mathrm{x}}$ and $\mathrm{TiH}_{\mathrm{x}}$ and mixed $\mathrm{Co}-\mathrm{TiO}_{\mathrm{x}}$ species in the database (or the $\mathrm{Si}$ analogues) were included as possible reaction products together with gaseous $\mathrm{H}_{2}, \mathrm{H}_{2} \mathrm{O}$ and $\mathrm{O}_{2}$. A large excess of $\mathrm{H}_{2}$ and $\mathrm{TiO}_{2}$ or $\mathrm{SiO}_{2}$ with respect to $\mathrm{CoO}$ was selected to keep the partial pressure of $\mathrm{H}_{2} \mathrm{O}$ formed upon reduction of $\mathrm{CoO}$ low, in line with the experimental conditions during reduction in a plug flow reactor.

Based on these calculations, it is thermodynamically possible to form small amounts of cobalt titanates in a reducing environment at the relevant temperatures, whilst cobalt silicates are not stable under the same conditions. N.B. $\mathrm{H}_{2}$ and the supports are outside of the scale of the plots. 

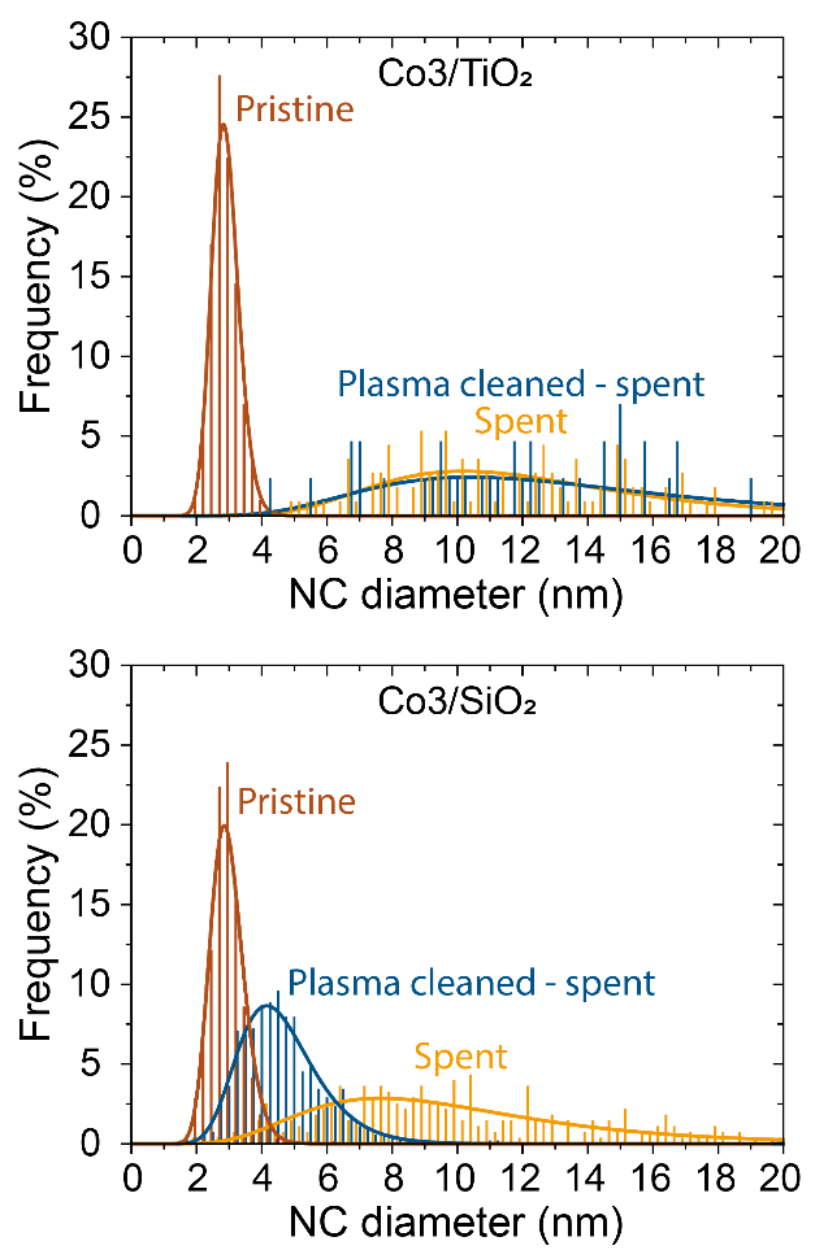

Figure C11. Effect of ligand removal on the particle growth in $\mathrm{Co} 3 / \mathrm{TiO}_{2}$ (left) and $\mathrm{Co} 3 / \mathrm{SiO}_{2}$ (right). The histograms and lognormal distributions are shown for the catalysts in the pristine, spent and cleaned and then spent state. Ligands were removed by plasma cleaning for $8 \mathrm{~h}$ prior to reduction and catalysis. No effect was observed on the particle size after catalysis on $\mathrm{TiO}_{2}$ but it did reduce the sintering in $\mathrm{Co} 3 / \mathrm{SiO}_{2}$. 

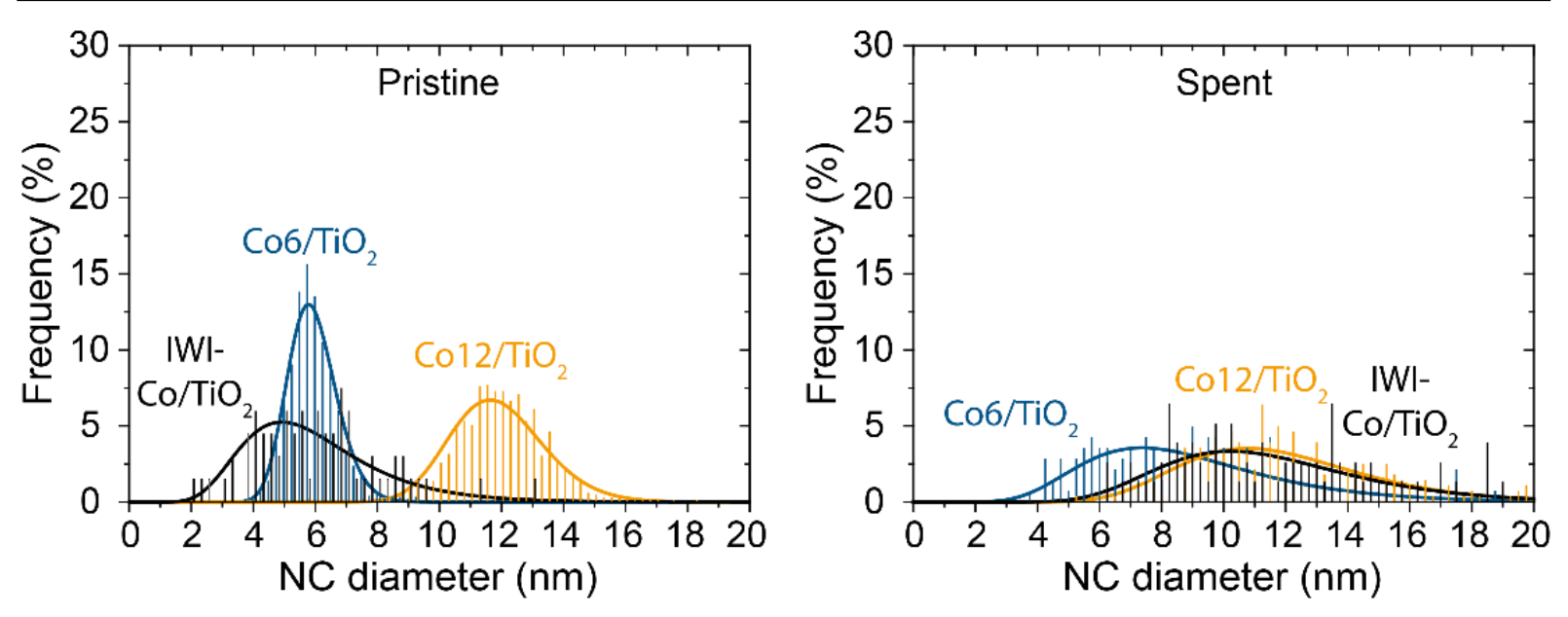

Figure C12. Sintering behavior of IWI-Co/ $/ \mathrm{TiO}_{2}$ after $>100 \mathrm{~h}$ on stream at $20 \mathrm{bar}, 220{ }^{\circ} \mathrm{C}, 2 \mathrm{H}_{2} / \mathrm{CO}$. The histograms and lognormal distributions of the $\mathrm{IWI}-\mathrm{Co} / \mathrm{TiO}_{2}$ before (left) and after catalysis (right) are shown together with the histograms and lognormal distributions for the $\mathrm{NC}-$ based $\mathrm{Co} / \mathrm{TiO}{ }_{2}$ and $\mathrm{Co} 12 / \mathrm{TiO}_{2}$ samples as comparison.

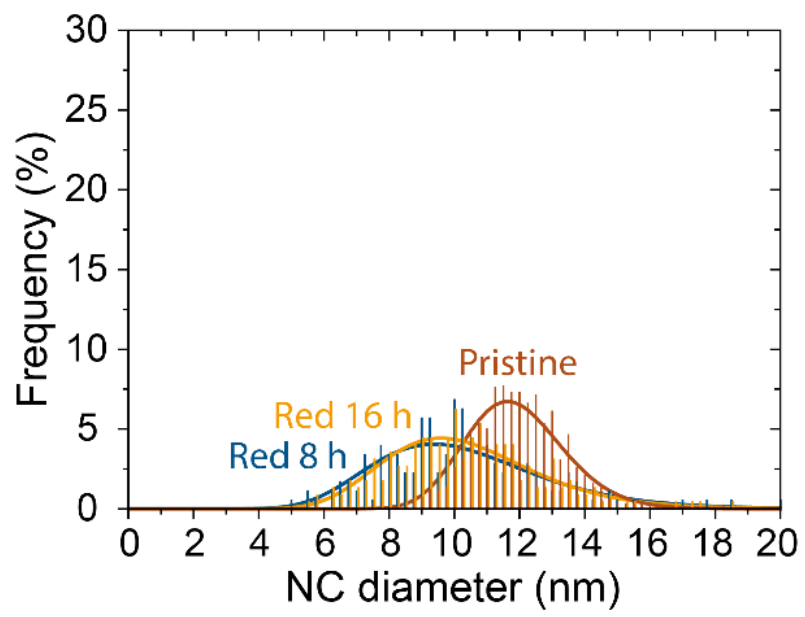

Figure C13. Effect of prolonged reduction on the particle growth in $\mathrm{Co12} / \mathrm{TiO}_{2}$. The histograms and lognormal distributions are shown for $\mathrm{Co1} 2 / \mathrm{TiO}_{2}$ in the pristine state and after reduction at $350{ }^{\circ} \mathrm{C}$ for 8 and $16 \mathrm{~h}$. Prolonged reduction did not influence the final particle size. 

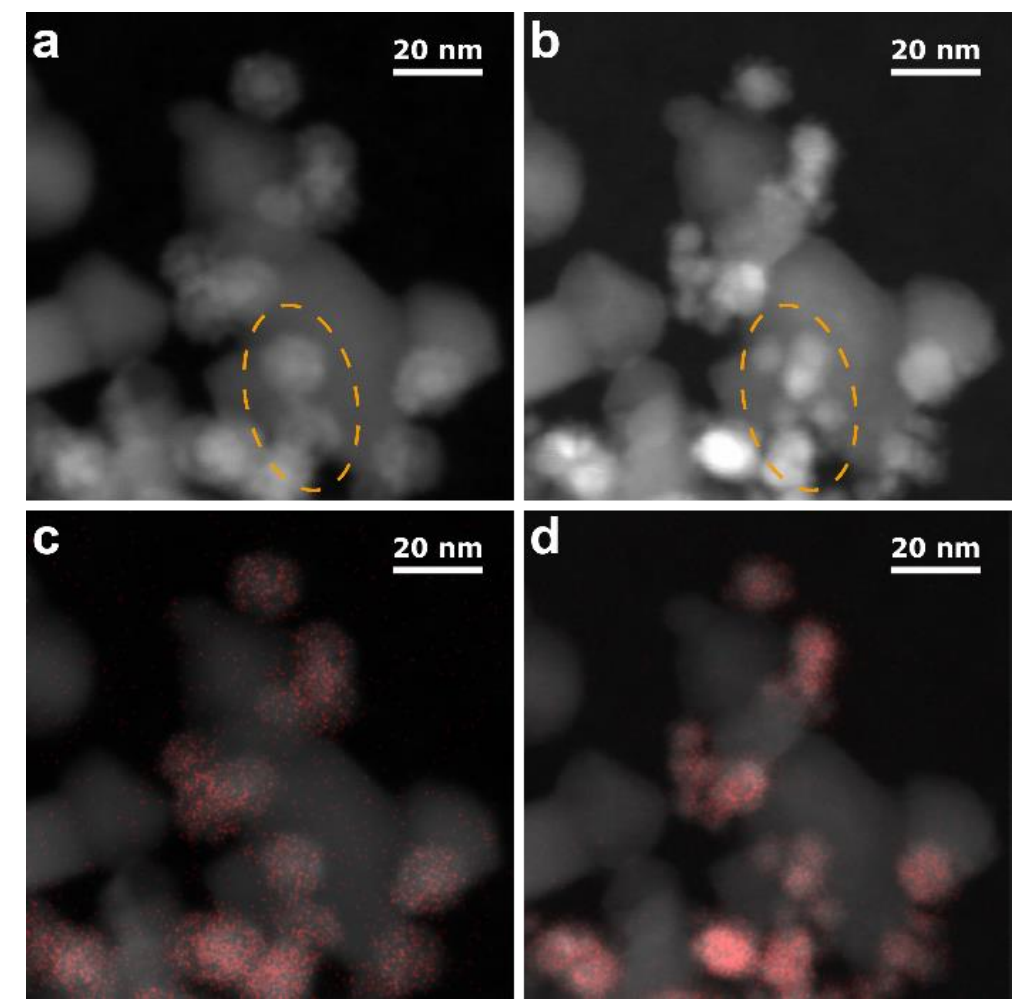

Figure C14. The size evolution of individual $\mathrm{Co}-\mathrm{NC}$ in Co12/TiO2 during reduction. a HAADF-STEM and c HAADF-STEM-EDX images of pristine Co12/TiO2. b HAADF-STEM and d HAADF-STEM-EDX images of the same locations after reduction at $350{ }^{\circ} \mathrm{C}$ for $8 \mathrm{~h}$. Co is depicted in red. The highlighted area contains two particles that disintegrated during reduction and formed multiple smaller particles. 


\section{Appendix D}

\section{Methods}

\section{In situ XRD quantitative phase analysis}

The diffractograms from in situ XRD were analyzed by Rietveld refinement using Bruker TOPAS v5.0 software. First, a $\mathrm{LaB}_{6}$ standard reference material (660c) from the National Institute of Standards and Technology was measured and fitted using the fundamental parameters approach to determine the emission profile and instrument details. The $\mathrm{K}$ emission profile of Mo was refined to $\mathrm{K}_{\alpha 1}$ emission of $0.708856 \AA$ and $\mathrm{K}_{\alpha 2}$ emission of $0.712992 \AA$, while simultaneously refining a $4^{\text {th }}$ order Chebyshev polynomial background, tube tails, cylindrical $2 \theta$ correction (Sabine), and sample displacement and tilt. The obtained emission profile and instrument details were applied in the analysis of all samples.

The bare support was measured to establish the contribution of the support as well as the background from the sample holder, e.g. the borosilicate capillary and quartz wool. The XRD profile was fitted between 7 and $37.5^{\circ} 2 \ominus$ using a $4^{\text {th }}$ order Chebyshev polynomial background and 8 pseudo-Voigt peaks. Simultaneously, cylindrical $2 \theta$ correction (Sabine) and sample displacement and tilt were refined.

The XRD patterns of the catalysts were fitted using the determined $\mathrm{SiO}_{2}$ contribution in combination with identified cobalt phases obtained from the ICDD PDF-4+2016 database, i.e. hcp Co (PDF 04-001-3273), fcc Co (PDF 04-001-2681) and CoO (PDF 04-0018626). The $\mathrm{SiO}_{2}$ contribution was predominantly fixed, leaving only the possibility for scaling. For the Co phases, the lattice parameters were constrained by $\pm 0.05 \AA$, although for most phases variations of $\pm 0.02 \AA$ were obtained. Size broadening was described using Lorentzian functions, while strain broadening was not included in the analysis, because that did not improve the quality of the fit. For hcp Co, preferred orientations in the [100] and [001] directions were also refined using the March-Dollase model. We tentatively propose that this is because of an hcp-fcc intergrowth phase that needs to be taken into account (see results and discussion in the main text). The average crystallite size was determined as the volume weighted mean column length, derived from the integral peak breadth using a crystallite shape factor $(\mathrm{k})$ of 1 . A $4^{\text {th }}$ order Chebyshev polynomial background, cylindrical $2 \theta$ correction (Sabine) and sample displacement and tilt were refined together with the $\mathrm{SiO}_{2}$ contribution and the Co phases. 


\section{Results}

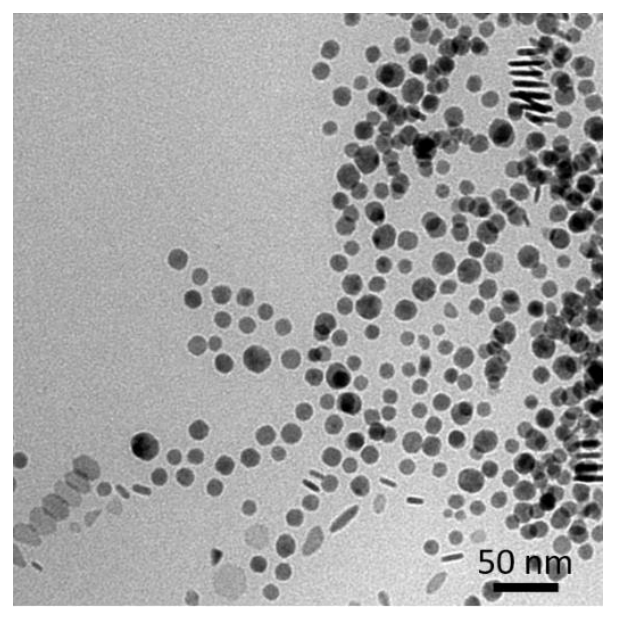

Figure D1. TEM image of the precipitate from magnetic separation directly after the synthesis of disk-shaped and spherical Co-NC. The rounded, high-contrast particles were classified as spherical $\mathrm{NC}$ and the low-contrast and elongated, high-contrast NC were classified as disk-shaped Co-NC. 

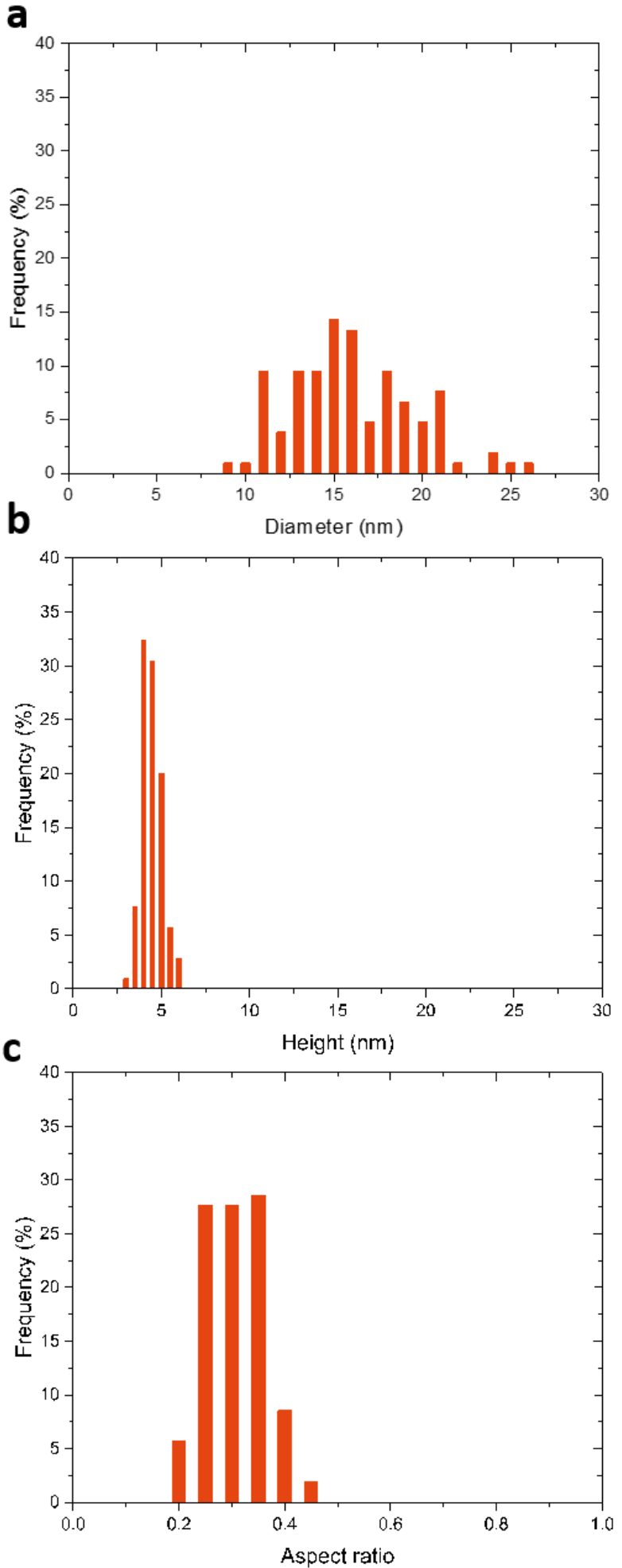

Figure D2. Size distributions of 100 disk-shaped Co-NC. Histogram of a the diameter, $\mathbf{b}$ the height and $\mathrm{c}$ the resulting aspect ratio of the $\mathrm{Co}-\mathrm{NC}$. 


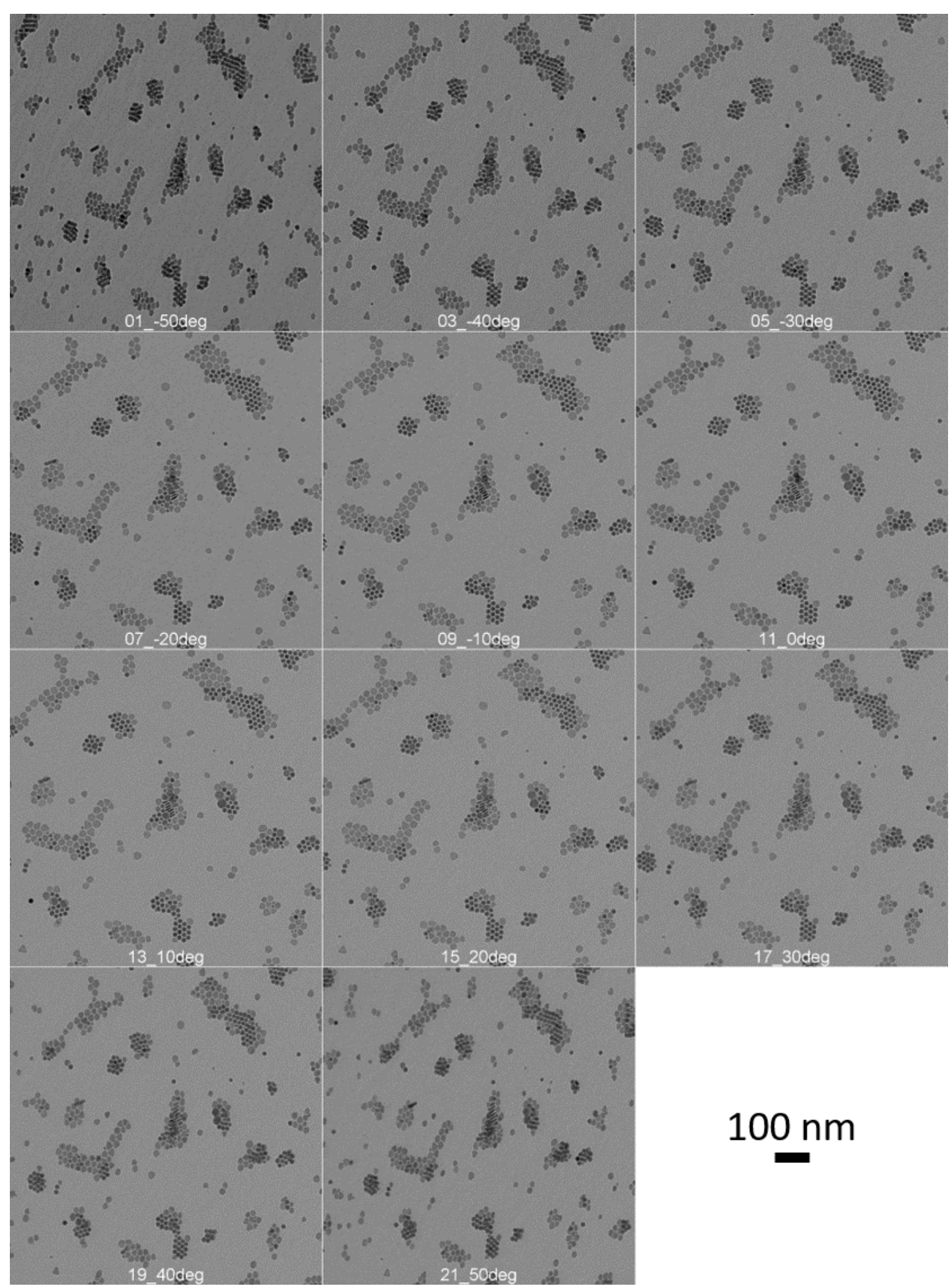

Figure D3. Tilting series of a TEM sample with disk-shaped and spherical CoO-NC between -50 and $50^{\circ}$ with an interval of $10^{\circ}$. 
a

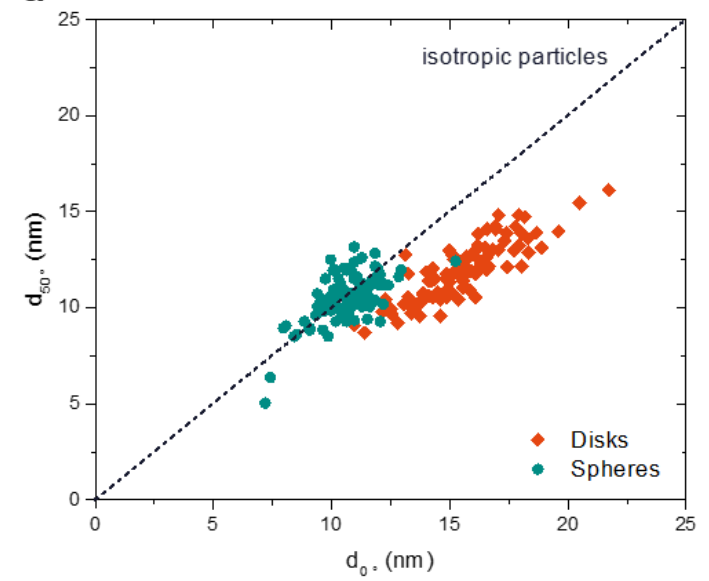

b

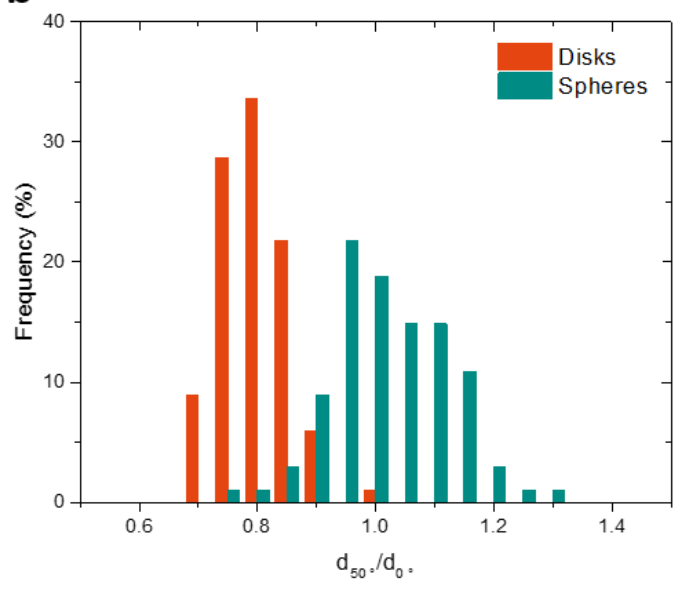

Figure D4. TEM analysis of the diameter of 100 disk-shaped and 100 spherical Co-NC before and after tilting the TEM sample by $50^{\circ}$. a the diameter at $50^{\circ}$ plotted against the diameter of the same $\mathrm{NC}$ at $0^{\circ}$ with the diagonal dashed line indicating an unchanged diameter. $\mathbf{b}$ Histogram of the diameter at $50^{\circ}\left(\mathrm{d}_{50}{ }^{\circ}\right)$ over the diameter at $0^{\circ}\left(\mathrm{d}_{0}{ }^{\circ}\right)$.

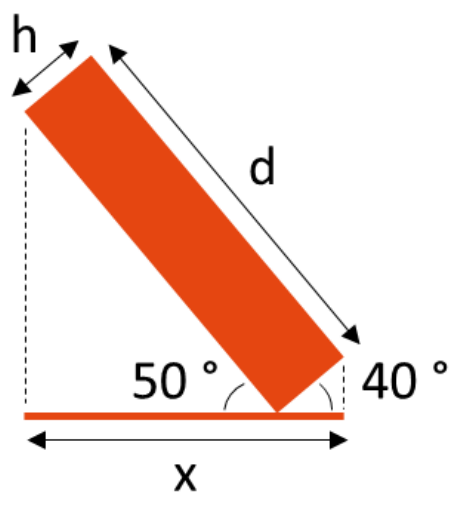

Figure D5. Illustration of the predicted projection length of a Co-NC disk tilted by $50^{\circ}$. The theoretical projection length at $50^{\circ}$ was calculated as $x=\cos \left(50^{\circ}\right) \cdot d+\cos \left(40^{\circ}\right) \cdot h$. 


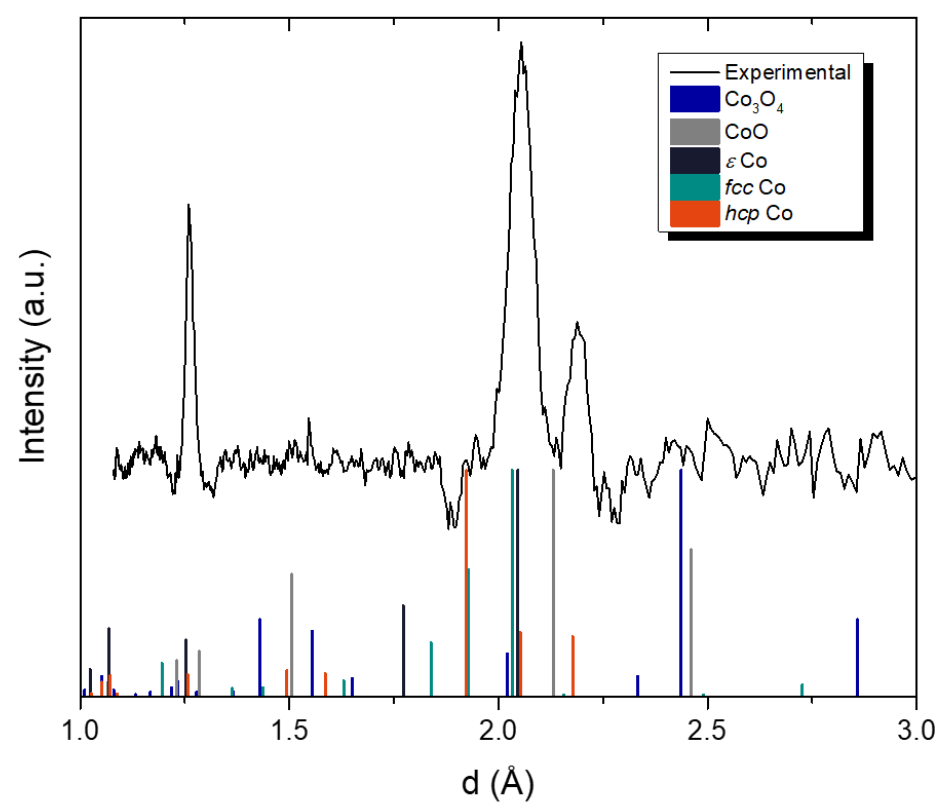

Figure D6. Intensity profile obtained by radially averaging and background correcting the SAED pattern from the inset of Figure 5.2a. The peak at $d=2.19 \AA$ could be assigned exclusively to hcp cobalt. The following reference diffraction patterns were obtained from the PDF-4+ 2016 database: fcc Co (00-015-0806), hep Co (01-071-4652), $\varepsilon$ Co (04-017-5578), $\mathrm{CoO}(00-042-1300), \mathrm{Co}_{3} \mathrm{O}_{4}(00-$ 042-1467)

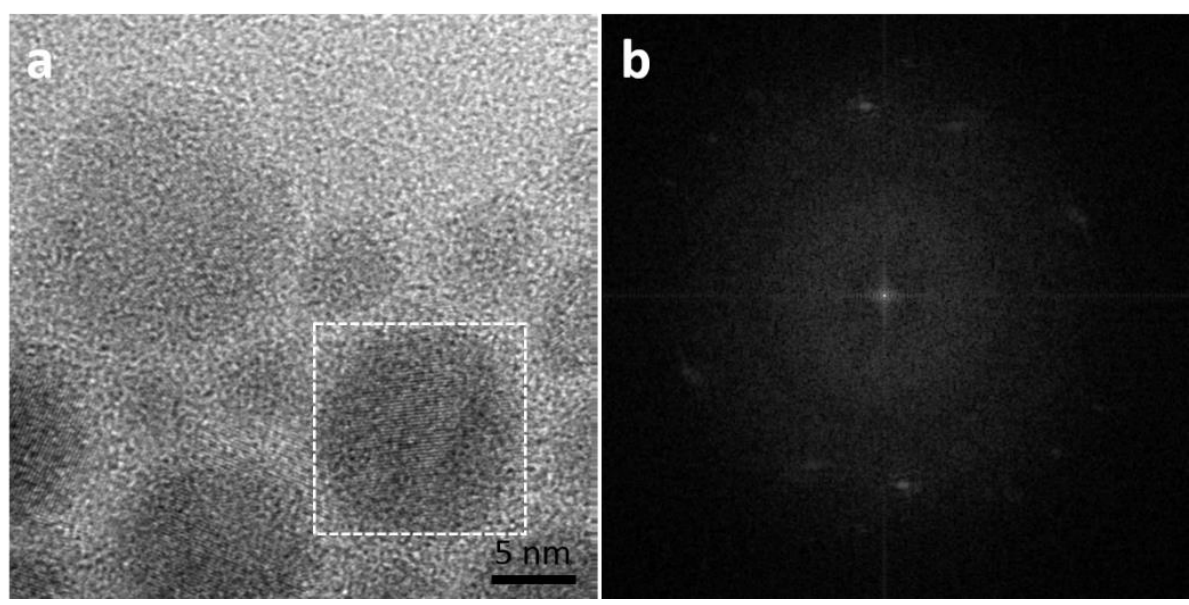

Figure D7. High-resolution TEM under cryogenic conditions of as-synthesized disk-shaped and spherical Co-NC with little to no solvent present. a TEM image with in the framed area an $11 \mathrm{~nm}$, high-contrast NC that was classified as spherical. $\mathbf{b}$ Fourier transform of the highlighted area. The lack of hexagonal symmetry together with the obtained lattice spacings $(2.14,1.93$ and $1.70 \AA)$ indicated that the particle consisted of $\varepsilon$-cobalt. 


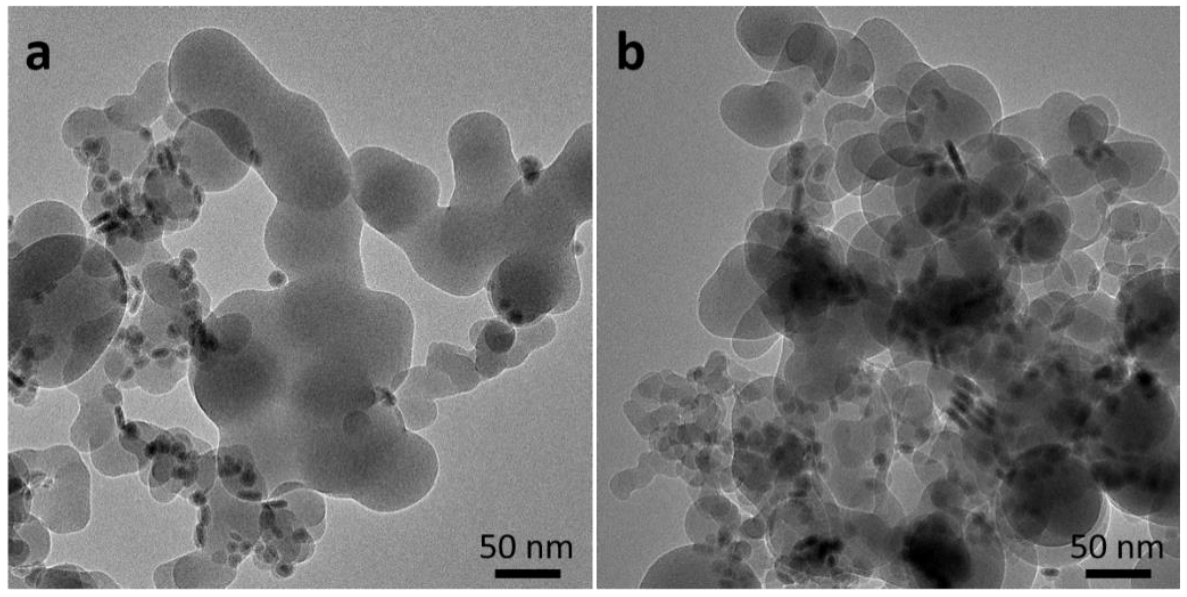

Figure D8. TEM images of a batch of mixture of disk-shaped and spherical Co-NC attached to $\mathrm{SiO}_{2}$. The Co underwent only one precipitation-redispersion cycle after synthesis and were attached to $\mathrm{SiO}_{2}$ afterwards. $\mathbf{a}$ and $\mathbf{b}$ are two images of this sample showing inhomogeneous distribution of the $\mathrm{Co}-\mathrm{NC}$.
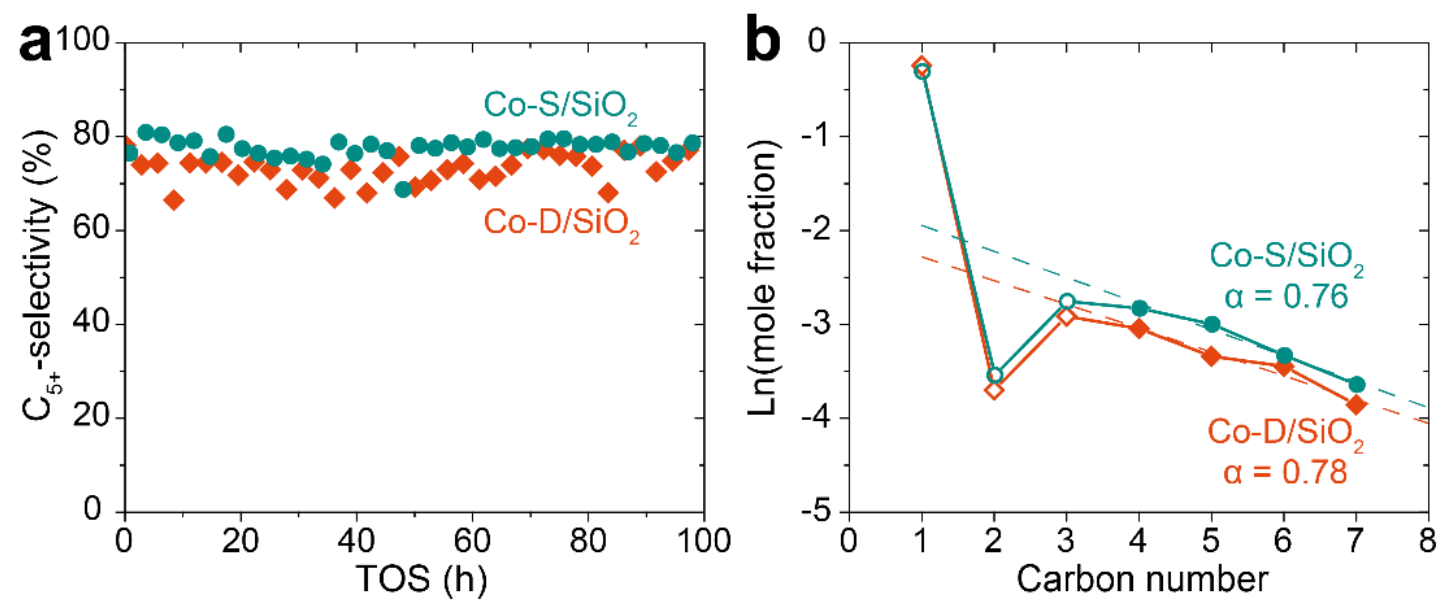

Figure D9. Fischer-Tropsch synthesis results of $\mathrm{Co}-\mathrm{D} / \mathrm{SiO}_{2}$ and $\mathrm{Co}-\mathrm{S} / \mathrm{SiO}_{2}$ at $20 \mathrm{bar}, 220{ }^{\circ} \mathrm{C}$ and $\mathrm{H}_{2} / \mathrm{CO}=2 \mathrm{v} / \mathrm{v}$. a $\mathrm{C}_{5+}-$ selectivity as a function of time-on-stream and $\mathbf{b}$ ASF product distribution averaged between $80-100 \mathrm{~h}$ on stream. The trendlines fitted to the solid filled datapoints $\left(\mathrm{C}_{4}-\mathrm{C}_{7}\right)$ were used to determine $\alpha$. 




Figure D10. XPS survey scans of the catalysts in the pristine and spent state and a bare $\mathrm{SiO}_{2}$ reference. The spent catalysts were measured after $100 \mathrm{~h}$ FT and subsequent passivation. The data was offset with $1 \cdot 10^{5} \mathrm{CPS}$ increment at $0 \mathrm{eV}$ binding energy. The regions of interest are labelled. 


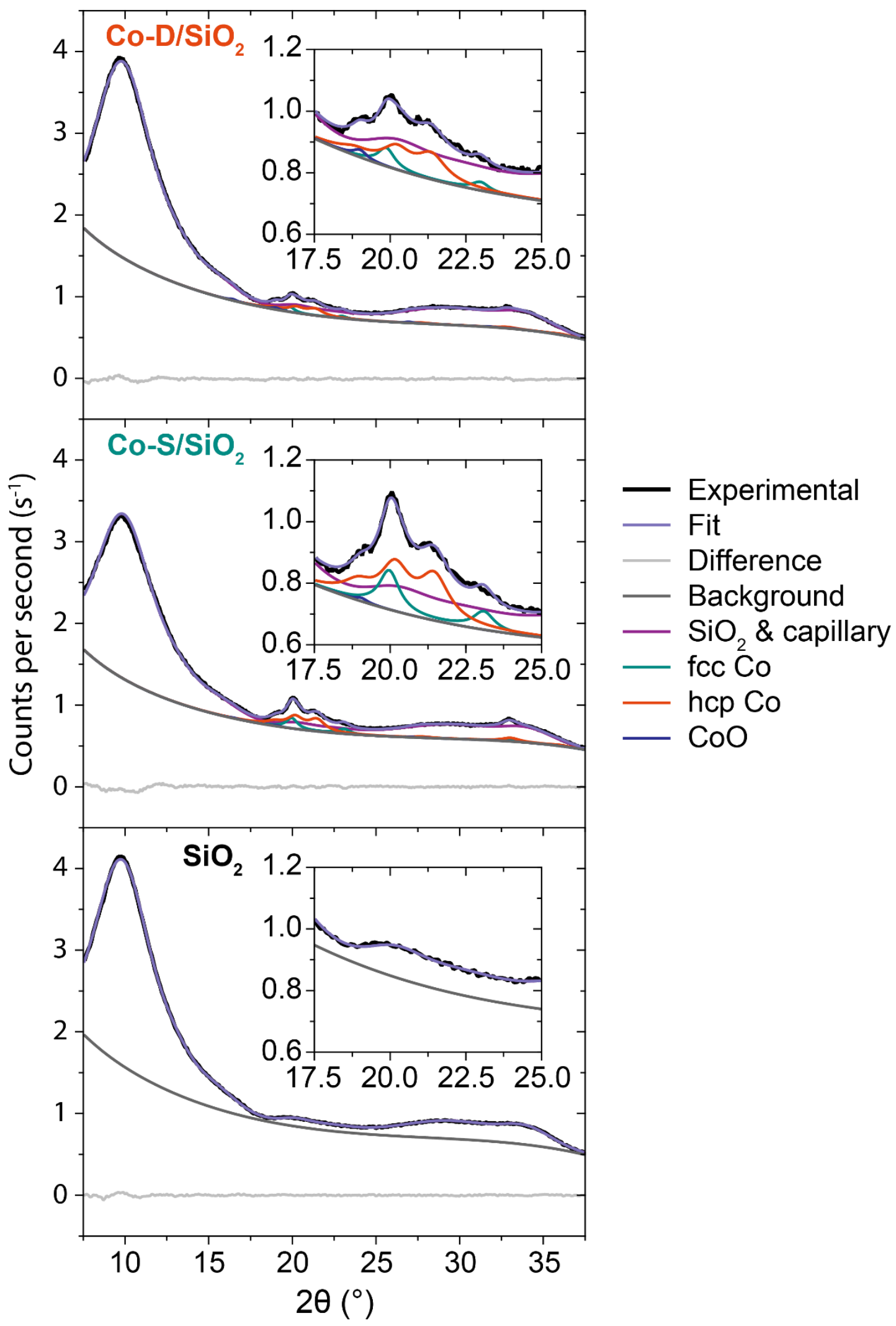

Figure D11. In situ XRD profiles and Rietveld refinement results of the $\mathrm{Co}-\mathrm{NC} / \mathrm{SiO}_{2}$ during FischerTropsch synthesis. The bare $\mathrm{SiO}_{2}$ support was measured under ambient conditions as a reference. The diffractograms were obtained over the first $18 \mathrm{~h}$ on stream at $10 \mathrm{bar}, 220^{\circ} \mathrm{C}$ and $\mathrm{H}_{2} / \mathrm{CO}=2 \mathrm{v} / \mathrm{v}$ by accumulating six scans of $3 \mathrm{~h}$. No change in the XRD patterns was observed over the course of the reaction. 


\section{Appendix E}
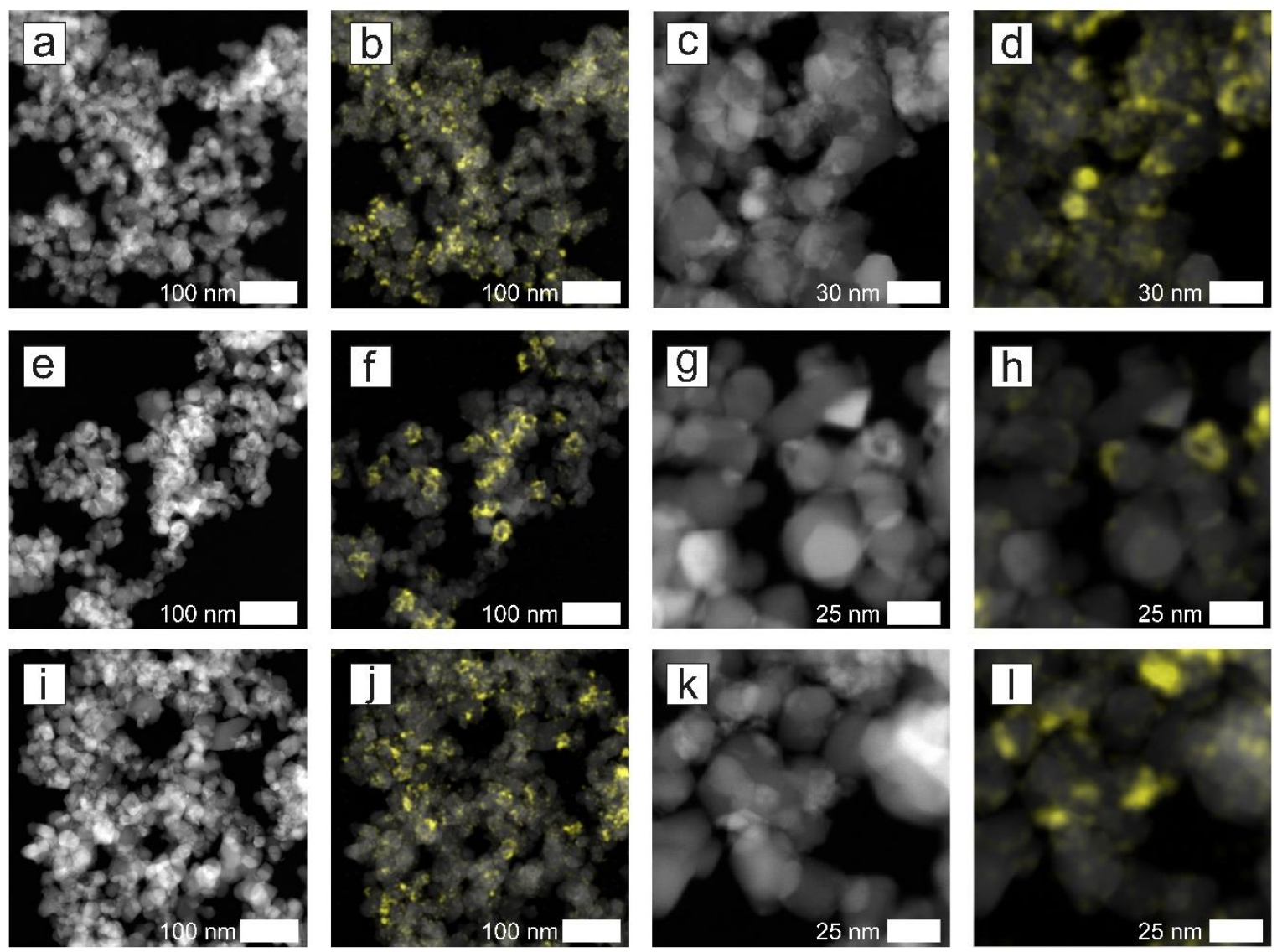

Figure E1. Additional electron microscopy images of the $\mathrm{TiO}_{2}$-supported samples upon reductionoxidation-reduction treatments. STEM-HAADF combined with EDX mapping of $\mathrm{Co} / \mathrm{TiO}_{2}$ at various stages of the ROR treatment. Co is depicted in yellow. a, b, c, d Samples after reduction at $350{ }^{\circ} \mathrm{C}$ and passivation $\left(\mathrm{R}-\mathrm{TiO}_{2}\right) ; \mathrm{e}, \mathrm{f}, \mathrm{g}, \mathrm{h}$ samples after reduction at $350{ }^{\circ} \mathrm{C}$ and oxidation at $200{ }^{\circ} \mathrm{C}(\mathrm{RO} 200-$ $\left.\mathrm{TiO}_{2}\right) ; \mathbf{i}, \mathbf{j}, \mathbf{k}, \mathbf{1}$ samples after reduction at $350{ }^{\circ} \mathrm{C}$, oxidation at $200{ }^{\circ} \mathrm{C}$ and reduction at $220{ }^{\circ} \mathrm{C}$ $\left(\mathrm{RO} 200 \mathrm{R}-\mathrm{TiO}_{2}\right)$ and passivation. 

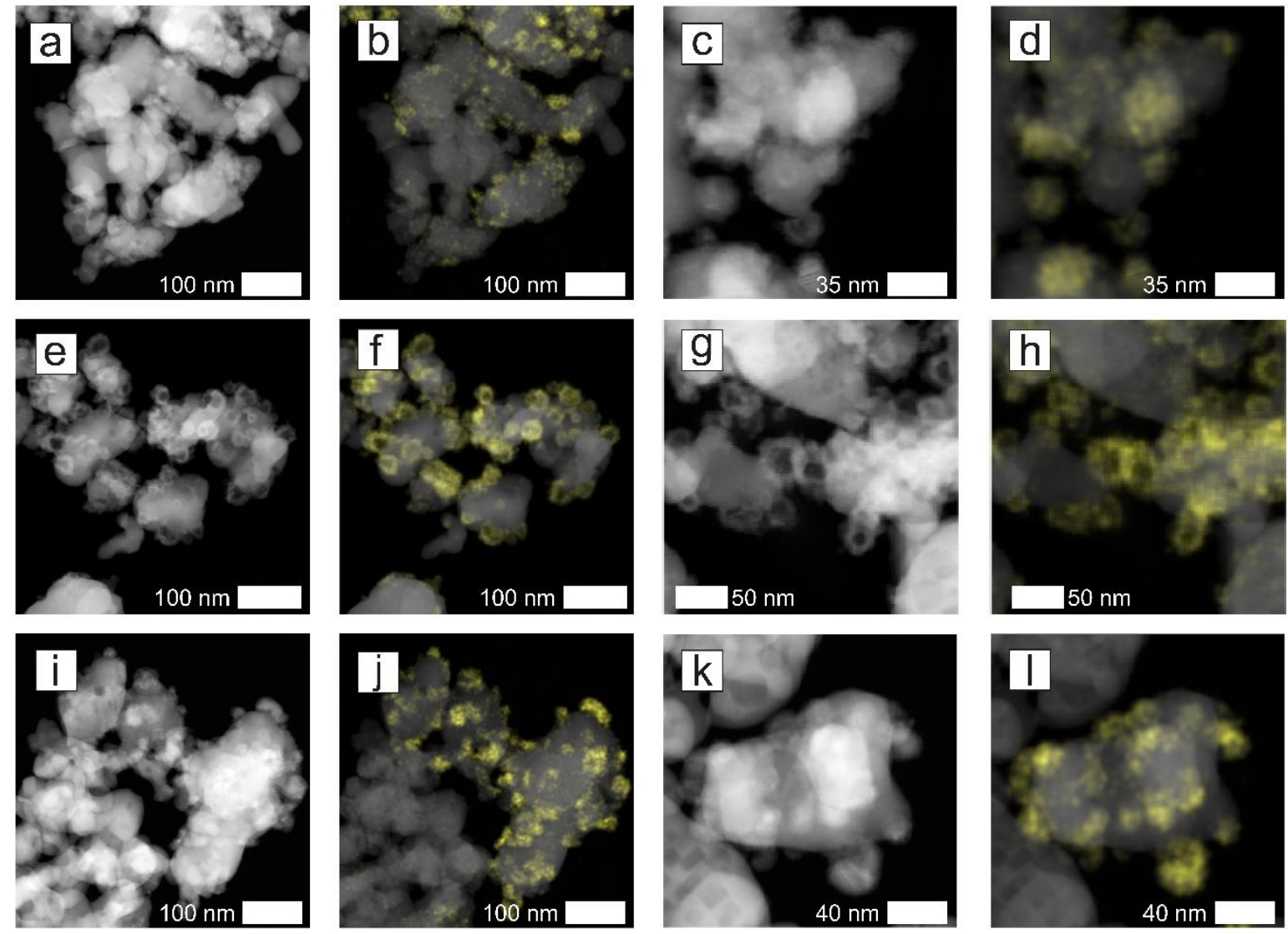

Figure E2. Additional electron microscopy images of the $\mathrm{Nb}_{2} \mathrm{O}_{5}$-supported samples upon reductionoxidation-reduction treatments. STEM-HAADF combined with EDX mapping of $\mathrm{Co} / \mathrm{Nb}_{2} \mathrm{O}_{5}$ at various stages of the ROR treatment. Co is depicted in yellow. $\mathbf{a}, \mathbf{b}, \mathbf{c}, \mathbf{d}$ Samples after reduction at $350{ }^{\circ} \mathrm{C}$ and passivation $\left(\mathrm{R}-\mathrm{Nb}_{2} \mathrm{O}_{5}\right) ; \mathrm{e}, \mathrm{f}, \mathrm{g}, \mathrm{h}$ samples after reduction at $350{ }^{\circ} \mathrm{C}$ and oxidation at $200{ }^{\circ} \mathrm{C}$ $\left(\mathrm{RO} 200-\mathrm{Nb}_{2} \mathrm{O}_{5}\right) ; \mathbf{i}, \mathbf{j}, \mathbf{k}, 1$ samples after reduction at $350{ }^{\circ} \mathrm{C}$, oxidation at $200{ }^{\circ} \mathrm{C}$ and reduction at $220{ }^{\circ} \mathrm{C}\left(\mathrm{RO} 200 \mathrm{R}-\mathrm{Nb}_{2} \mathrm{O}_{5}\right)$ and passivation. 

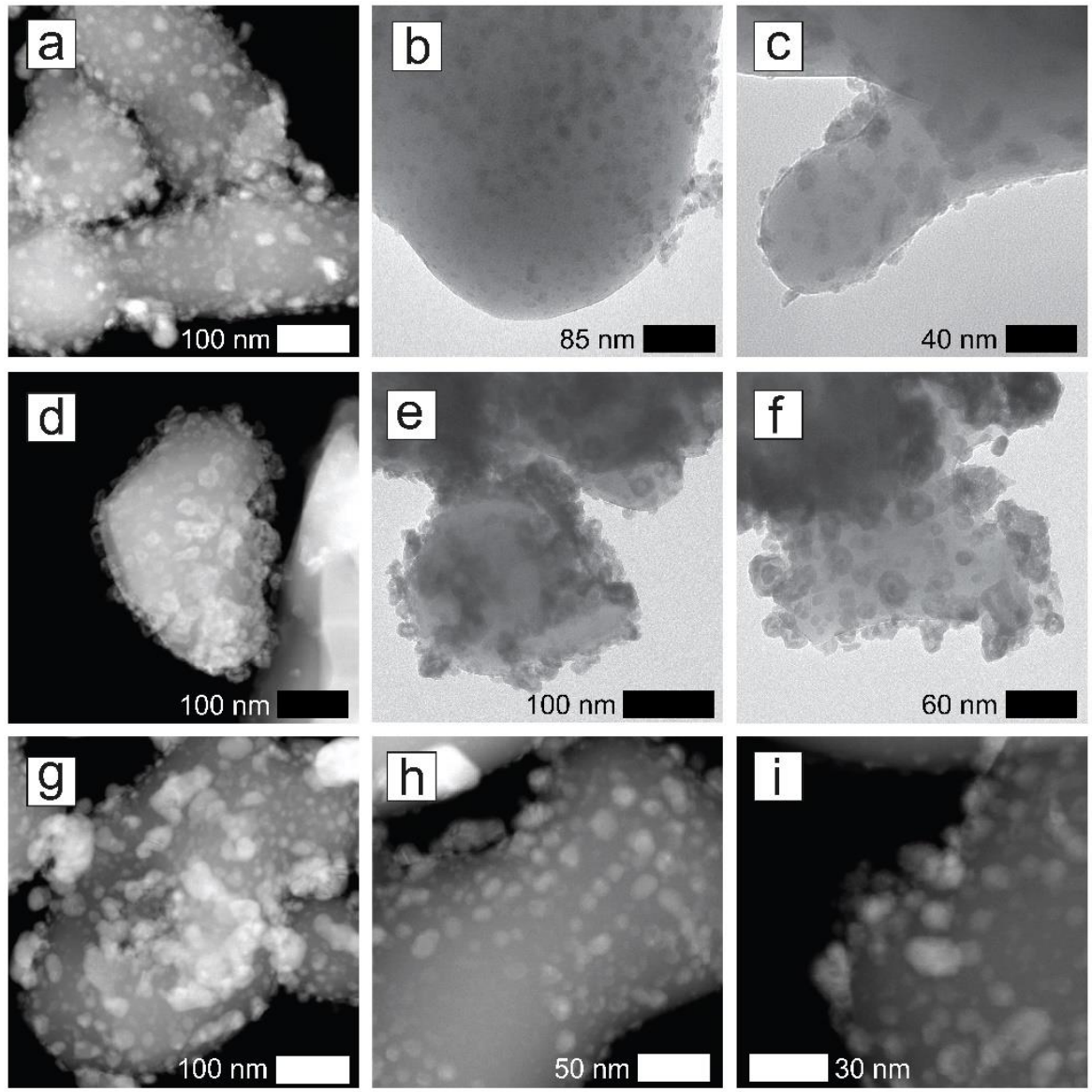

Figure E3. Additional electron microscopy images of the $\mathrm{Al}_{2} \mathrm{O}_{3}$-supported samples upon reductionoxidation-reduction treatments. a, d, g, h, i STEM-HAADF and b, c, e, $\mathbf{f}$ bright-field TEM of $\mathrm{Co} / \mathrm{Al}_{2} \mathrm{O}_{3}$ at various stages of the ROR treatment. a, b, c Samples after reduction at $350{ }^{\circ} \mathrm{C}$ and passivation $\left(\mathrm{R}-\mathrm{Al}_{2} \mathrm{O}_{3}\right)$; d, e, f samples after reduction at $350{ }^{\circ} \mathrm{C}$ and oxidation at $200{ }^{\circ} \mathrm{C}(\mathrm{RO} 200-$ $\left.\mathrm{Al}_{2} \mathrm{O}_{3}\right) ; \mathbf{g}, \mathbf{h}, \mathbf{i}$, samples after reduction at $350{ }^{\circ} \mathrm{C}$, oxidation at $200{ }^{\circ} \mathrm{C}$ and reduction at $220{ }^{\circ} \mathrm{C}$ $\left(\mathrm{RO} 200 \mathrm{R}-\mathrm{Al}_{2} \mathrm{O}_{3}\right.$ ) and passivation. 
Table E1. Cobalt loading of $\mathrm{Co} / \mathrm{TiO}_{2}, \mathrm{Co} / \mathrm{Nb}_{2} \mathrm{O}_{5}$ and $\mathrm{Co} / \alpha-\mathrm{Al}_{2} \mathrm{O}_{3}$, as determined by inductively coupled plasma-optical emission spectroscopy (ICP-OES).

\begin{tabular}{ll}
\hline Sample & $\begin{array}{l}\text { Cobalt loading } \\
(\text { wt.\%) }\end{array}$ \\
\hline $\mathrm{Co} / \mathrm{TiO}_{2}$ & 6.8 \\
$\mathrm{Co} / \mathrm{Nb}_{2} \mathrm{O}_{5}$ & 5.7 \\
$\mathrm{Co} / \alpha-\mathrm{Al}_{2} \mathrm{O}_{3}$ & 6.0 \\
\hline
\end{tabular}

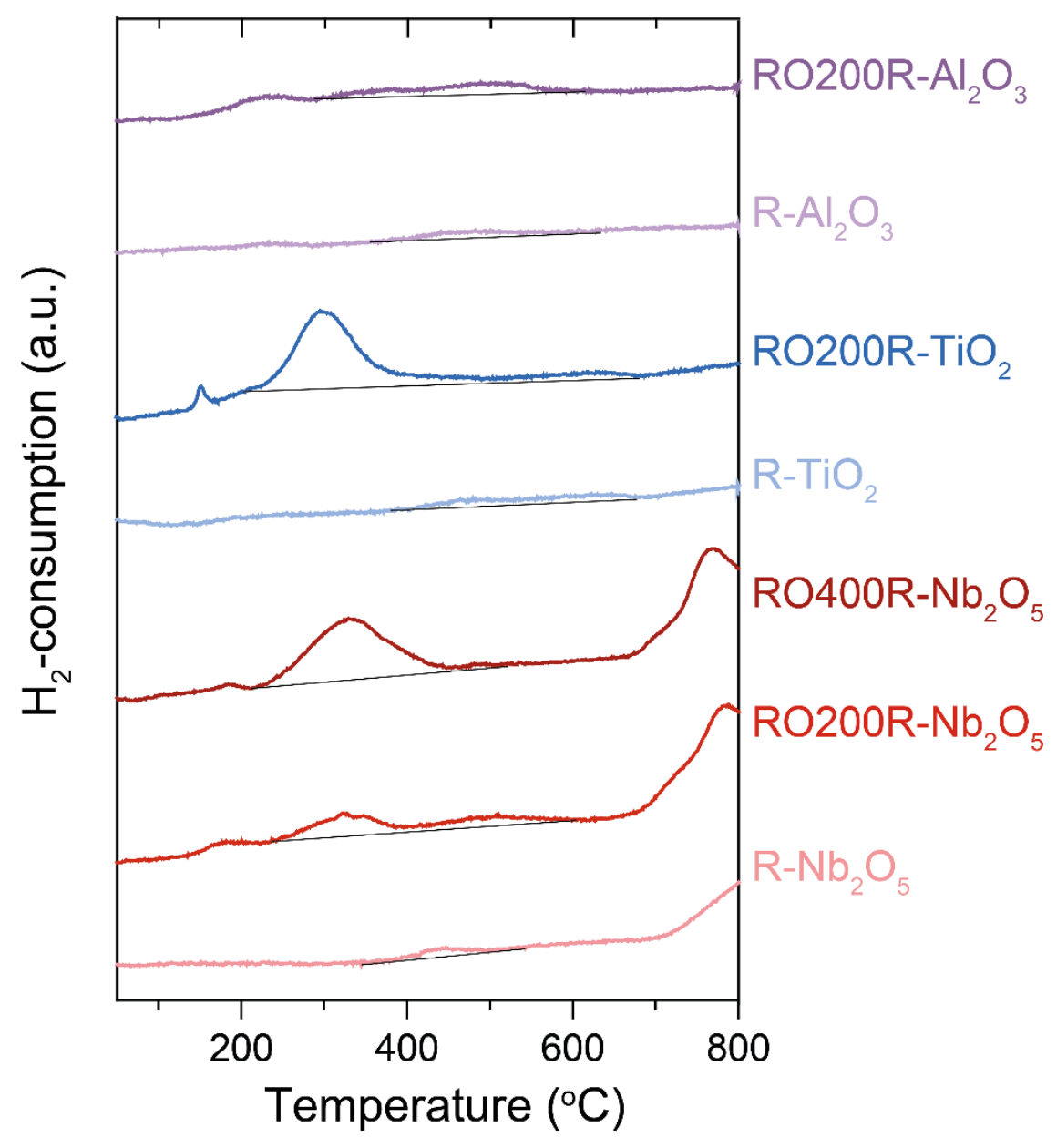

Figure E4. TPR profiles used to determine the degree of reduction of cobalt. The baseline is shown for the peaks that were integrated as cobalt that had not been reduced and thus lowered the degree of reduction. The degree of reduction was determined after reduction at $350{ }^{\circ} \mathrm{C}$ (R-samples), after reduction at $350{ }^{\circ} \mathrm{C}$, oxidation at $200^{\circ} \mathrm{C}$ and reduction at $220^{\circ} \mathrm{C}$ (RO200R-samples) and after reduction at $350{ }^{\circ} \mathrm{C}$, oxidation at $400{ }^{\circ} \mathrm{C}$ and reduction at $220{ }^{\circ} \mathrm{C}\left(\mathrm{RO} 400 \mathrm{R}-\mathrm{Nb}_{2} \mathrm{O}_{5}\right)$. 
Table E2. The degree of cobalt reduction at various stages of the ROR treatment, as measured by TPR. The degree of reduction was determined after reduction at $350^{\circ} \mathrm{C}(\mathrm{R}$-samples), after reduction at $350{ }^{\circ} \mathrm{C}$, oxidation at $200{ }^{\circ} \mathrm{C}$ and reduction at $220{ }^{\circ} \mathrm{C}$ (RO200R-samples) and after reduction at $350{ }^{\circ} \mathrm{C}$, oxidation at $400{ }^{\circ} \mathrm{C}$ and reduction at $220{ }^{\circ} \mathrm{C}\left(\mathrm{RO} 400 \mathrm{R}-\mathrm{Nb}_{2} \mathrm{O}_{5}\right)$.

\begin{tabular}{llll}
\hline Sample & \multicolumn{3}{c}{ DOR $(\%)$} \\
\cline { 2 - 4 } & $\mathrm{R}$ & RO200R & RO400R \\
\hline $\mathrm{Co} / \mathrm{TiO}_{2}$ & 97 & 86 & n.d. \\
$\mathrm{Co} / \mathrm{Nb}_{2} \mathrm{O}_{5}$ & 98 & 93 & 84 \\
$\mathrm{Co} / \mathrm{Al}_{2} \mathrm{O}_{3}$ & 98 & 95 & n.d. \\
\hline
\end{tabular}



Figure E5. Particle growth at higher oxidation temperature. Histogram of particle sizes of RO200R$\mathrm{TiO}_{2}$ and $\mathrm{RO} 400 \mathrm{R}-\mathrm{TiO}_{2}$. The samples were reduced under hydrogen flow at $220^{\circ} \mathrm{C}$ and then exposed to air at room temperature. Afterwards, the samples were analyzed by STEM-EDX mapping. 
$\mathrm{Co} / \mathrm{TiO}_{2}$

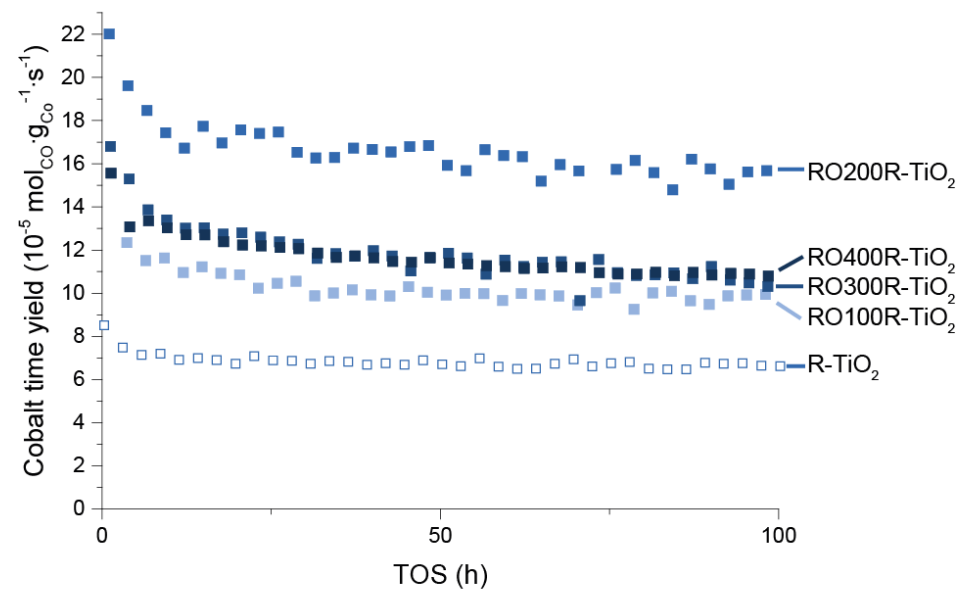

$\mathrm{Co} / \mathrm{Nb}_{2} \mathrm{O}_{5}$

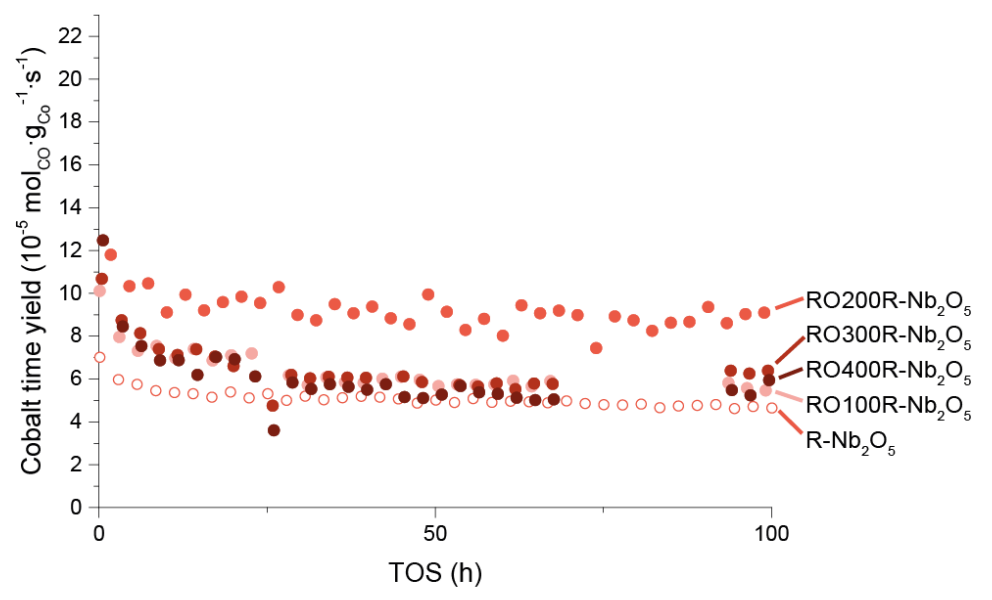

$\mathrm{Co} / \boldsymbol{\alpha}-\mathrm{Al}_{2} \mathrm{O}_{3}$

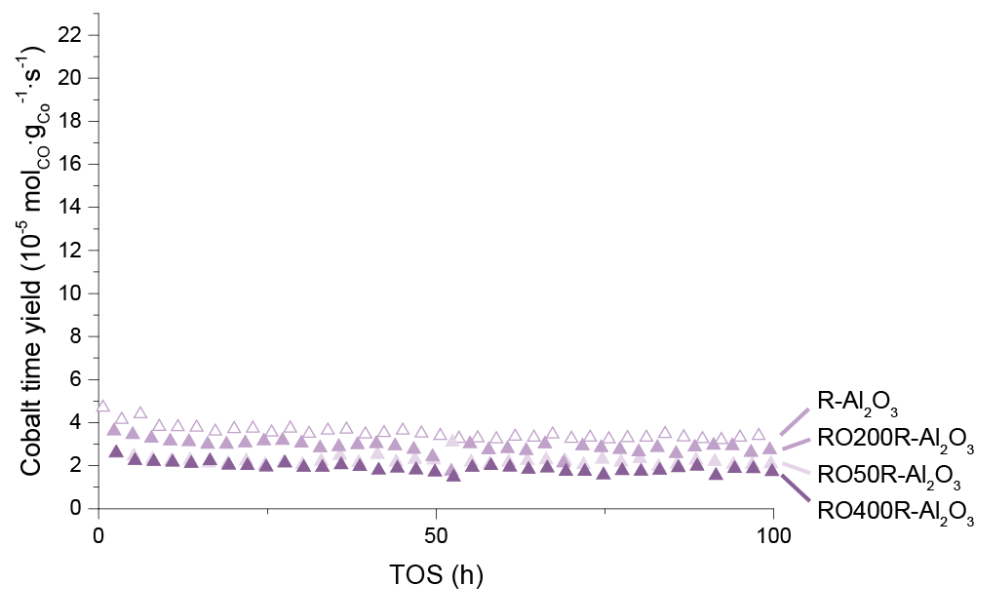

Figure E6. Cobalt-weight-based activity over time-on-stream. Cobalt-weight-based catalytic activity of $\mathrm{Co} / \mathrm{TiO}_{2}, \mathrm{Co} / \mathrm{Nb}_{2} \mathrm{O}_{5}$ and $\mathrm{Co} / \mathrm{Al}_{2} \mathrm{O}_{3}$ after various reduction-oxidation-reduction treatments plotted against time-on-stream. Reaction conditions: 20 bar, $220{ }^{\circ} \mathrm{C}, \mathrm{H}_{2} / \mathrm{CO}=2 \mathrm{~V} / \mathrm{V}, \mathrm{GHSV}=1000-$ $13000 \mathrm{~h}^{-1}$ and CO conversion $=15-34 \%\left(\mathrm{TiO}_{2}\right), 17-25 \%\left(\mathrm{Nb}_{2} \mathrm{O}_{5}\right), 8-12 \%\left(\mathrm{Al}_{2} \mathrm{O}_{3}\right)$. 
Table E3. Summary of the catalytic performance at $20 \mathrm{bar}, 220{ }^{\circ} \mathrm{C}$ and $\mathrm{H}_{2} / \mathrm{CO}=2 \mathrm{~V} / \mathrm{V}$. The reported data was averaged over three data points between $90-100 \mathrm{~h}$ on stream.

\begin{tabular}{|c|c|c|c|c|c|c|c|}
\hline \multirow[t]{2}{*}{ Sample } & \multirow{2}{*}{$\begin{array}{l}\text { GHSV } \\
\left(\mathbf{h}^{-1}\right)\end{array}$} & \multirow{2}{*}{$\begin{array}{l}\mathrm{CO} \\
\text { conv. } \\
(\%)\end{array}$} & \multirow{2}{*}{$\begin{array}{l}\text { Cobalt- } \\
\text { Time-- } \\
\text { Yield }^{\mathrm{a}}\end{array}$} & \multirow{2}{*}{$\begin{array}{l}\text { TOF } \\
\left(s^{-1}\right)\end{array}$} & \multicolumn{3}{|c|}{ Selectivity (\%) } \\
\hline & & & & & $\mathrm{C}_{1}$ & $\mathrm{C}_{2-4}$ & $\mathrm{C}_{5+}$ \\
\hline \multicolumn{8}{|l|}{$\mathrm{TiO}_{2}$} \\
\hline $\mathrm{R}-\mathrm{TiO}_{2}$ & 5560 & 20.6 & 6.7 & 0.10 & 8.5 & 7.5 & 84.0 \\
\hline RO100R-TiO 2 & 8290 & 18.2 & 9.8 & 0.11 & 7.0 & 5.0 & 88.0 \\
\hline $\mathrm{RO} 200 \mathrm{R}-\mathrm{TiO}_{2}$ & 13540 & 19.3 & 15.7 & 0.10 & 7.5 & 4.5 & 88.0 \\
\hline $\mathrm{RO} 300 \mathrm{R}-\mathrm{TiO}_{2}$ & 13540 & 15.1 & 10.5 & 0.074 & 8.8 & 5.4 & 85.8 \\
\hline $\mathrm{RO} 400 \mathrm{R}-\mathrm{TiO}_{2}$ & 5640 & 34.0 & 10.8 & 0.11 & 6.8 & 4.7 & 88.5 \\
\hline \multicolumn{8}{|l|}{$\mathrm{Nb}_{2} \mathrm{O}_{5}$} \\
\hline $\mathrm{R}-\mathrm{Nb}_{2} \mathrm{O}_{5}$ & 3266 & 17.0 & 4.7 & 0.087 & 8.6 & 6.1 & 85.3 \\
\hline $\mathrm{RO} 100 \mathrm{R}-\mathrm{Nb}_{2} \mathrm{O}_{5}$ & 3024 & 19.6 & 5.6 & 0.051 & 7.8 & 3.9 & 88.3 \\
\hline $\mathrm{RO} 200 \mathrm{R}-\mathrm{Nb}_{2} \mathrm{O}_{5}$ & 3874 & 24.6 & 8.9 & 0.068 & 6.7 & 4.2 & 89.1 \\
\hline $\mathrm{RO} 300 \mathrm{R}-\mathrm{Nb}_{2} \mathrm{O}_{5}$ & 3070 & 25.5 & 6.2 & 0.058 & 8.5 & 3.6 & 87.9 \\
\hline $\begin{array}{l}\mathrm{RO} 400 \mathrm{R}-\mathrm{Nb}_{2} \mathrm{O}_{5} \\
\boldsymbol{\alpha}-\mathrm{Al}_{2} \mathrm{O}_{3}\end{array}$ & 2954 & 19.3 & 5.5 & 0.077 & 11.4 & 4.6 & 85.0 \\
\hline $\mathrm{R}-\mathrm{Al}_{2} \mathrm{O}_{3}$ & 1249 & 12.5 & 3.3 & 0.025 & 10.9 & 14.2 & 85.8 \\
\hline $\mathrm{RO} 50 \mathrm{R}-\mathrm{Al}_{2} \mathrm{O}_{3}$ & 940 & 9.62 & 2.0 & 0.031 & 5.8 & 4.4 & 89.8 \\
\hline $\mathrm{RO} 200 \mathrm{R}-\mathrm{Al}_{2} \mathrm{O}_{3}$ & 952 & 8.98 & 2.8 & 0.035 & 6.2 & 4.1 & 89.7 \\
\hline $\mathrm{RO} 400 \mathrm{R}-\mathrm{Al}_{2} \mathrm{O}_{3}$ & 956 & 8.17 & 1.7 & 0.029 & 7.6 & 6.0 & 86.4 \\
\hline
\end{tabular}





Figure E7. Literature overview of cobalt-weight-based activities as a function of $\mathrm{H}_{2}$-uptake. Values obtained from literature were divided into two plots: a shows cobalt-based catalysts supported on reducible oxides $\left(\mathrm{TiO}_{2}\right.$ and $\left.\mathrm{Nb}_{2} \mathrm{O}_{5}\right)$ and $\mathbf{b}$ shows cobalt-based catalysts supported on irreducible oxides $\left(\alpha-\mathrm{Al}_{2} \mathrm{O}_{3}, \gamma-\mathrm{Al}_{2} \mathrm{O}_{3}\right.$ and $\left.\mathrm{SiO}_{2}\right)$. Open symbols refer to non-promoted, pristine cobalt catalysts and solid symbols to cobalt catalysts with enhanced activity either via noble metal/Mn promotion or via an ROR treatment (this work). $\mathrm{H}_{2}$-uptake values were calculated based on the reported particle sizes from either $\mathrm{H}_{2}$-chemisorption (refs., ${ }^{7}$ ), TEM or XRD. Activity values were recalculated to match the units reported here. FT was performed at 20 bar and $\mathrm{H}_{2} / \mathrm{CO}=2 \mathrm{~V} / \mathrm{V}$ in all cases, except ref. ${ }^{9}$ where $\mathrm{H}_{2} / \mathrm{CO}=1 \mathrm{~V} / \mathrm{V}$. When the catalytic performance was reported at a temperature different than $220^{\circ} \mathrm{C}$, the activity was recalculated to that at $220^{\circ} \mathrm{C}$ using the Arrhenius equation with an apparent activation energy of $100 \mathrm{~kJ} \cdot \mathrm{mol}^{-1}$. 
Table E4. Lewis acidity of various metal oxides. Data was obtained from ref ${ }^{14}$

\begin{tabular}{lll}
\hline $\begin{array}{l}\text { Formal oxidation state of } \\
\text { the cation }(\mathrm{Nm})\end{array}$ & $\begin{array}{l}\text { Formula of the } \\
\text { corresponding metal oxide }\end{array}$ & $\begin{array}{l}\text { Lewis acid character } \\
\left(\mathbf{N}_{\mathbf{M}}-\mathbf{2}_{\mathbf{M}}\right)^{\text {a }}\end{array}$ \\
\hline $5^{+}$ & $\mathrm{Nb}_{2} \mathrm{O}_{5}$ & 3.895 \\
$4^{+}$ & $\mathrm{TiO}_{2}$ & 3.046 \\
$\mathrm{NbO}_{2}$ & 2.877 \\
$3^{+}$ & $\mathrm{Ti}_{2} \mathrm{O}_{3}$ & 1.930 \\
& $\mathrm{Nb}_{2} \mathrm{O}_{3}$ & 1.883 \\
$\mathrm{Al}_{2} \mathrm{O}_{3}$ & 2.274 \\
\hline
\end{tabular}

${ }^{a} \delta_{M}$ corresponds to the Sanderson's partial charge of the cation.

\section{References}

1. Eschemann, T. O., Oenema, J. \& de Jong, K. P. Effects of noble metal promotion for $\mathrm{Co} / \mathrm{TiO}_{2}$ Fischer-Tropsch catalysts. Catal. Today 261, 60-66 (2016).

2. Eschemann, T. O. \& de Jong, K. P. Deactivation Behavior of $\mathrm{Co} / \mathrm{TiO}_{2}$ Catalysts during FischerTropsch Synthesis. ACS Catal. 5, 3181-3188 (2015).

3. Prieto, G. et al. Cobalt-Catalyzed Fischer-Tropsch Synthesis: Chemical Nature of the Oxide Support as a Performance Descriptor. ACS Catal. 5, 3323-3335 (2015).

4. Lögdberg, S. et al. Effect of water on the space-time yield of different supported cobalt catalysts during Fischer-Tropsch synthesis. Appl. Catal. A Gen. 393, 109-121 (2011).

5. Storsæeter, S., Borg, Ø., Blekkan, E. A. \& Holmen, A. Study of the effect of water on FischerTropsch synthesis over supported cobalt catalysts. J. Catal. 231, 405-419 (2005).

6. den Otter, J. H., Yoshida, H., Ledesma, C., Chen, D. \& de Jong, K. P. On the superior activity and selectivity of PtCo/ $\mathrm{Nb}_{2} \mathrm{O}_{5}$ Fischer Tropsch catalysts. J. Catal. 340, 270-275 (2016).

7. Iglesia, E. Design, synthesis, and use of cobalt-based Fischer-Tropsch synthesis catalysts. Appl. Catal. A Gen. 161, 59-78 (1997).

8. Hernández Mejía, C., den Otter, J. H., Weber, J. L. \& de Jong, K. P. Crystalline niobia with tailored porosity as support for cobalt catalysts for the Fischer-Tropsch synthesis. Appl. Catal. A Gen. 548, 143-149 (2017).

9. Sun, X. et al. Manufacture of highly loaded silica-supported cobalt Fischer-Tropsch catalysts from a metal organic framework. Nat. Commun. 8, 1680 (2017).

10. Cheng, K. et al. The role of carbon pre-coating for the synthesis of highly efficient cobalt catalysts for Fischer-Tropsch synthesis. J. Catal. 337, 260-271 (2016).

11. Johnson, G. R., Werner, S. \& Bell, A. T. An Investigation into the Effects of Mn Promotion on the Activity and Selectivity of $\mathrm{Co} / \mathrm{SiO}_{2}$ for Fischer-Tropsch Synthesis: Evidence for Enhanced CO Adsorption and Dissociation. ACS Catal. 5, 5888-5903 (2015).

12. Nabaho, D., Niemantsverdriet, J. W., Claeys, M. \& Van Steen, E. Hydrogen spillover in the Fischer-Tropsch synthesis: An analysis of gold as a promoter for cobalt-alumina catalysts. Catal. Today 275, 27-34 (2016).

13. Jermwongratanachai, T. et al. Fischer-Tropsch synthesis: Comparisons between Pt and Ag promoted $\mathrm{Co} / \mathrm{Al}_{2} \mathrm{O}_{3}$ catalysts for reducibility, local atomic structure, catalytic activity, and oxidation-reduction (OR) cycles. Appl. Catal. A Gen. 464-465, 165-180 (2013). 
14. Jeong, N. C. et al. Acidity Scale for Metal Oxides and Sanderson's Electronegativities of Lanthanide Elements. Angew. Chemie Int. Ed. 47, 10128 -10132 (2008). 


\section{List of publications and presentations}

\section{Publications}

van Deelen, T. W., Harmel, J. M., Nijhuis, J. J., Su, H., Yoshida, H., Oord, R., Zečević, J., Weckhuysen, B. M. \& de Jong, K. P. Disk-shaped Cobalt Nanocrystals as Fischer-Tropsch Synthesis Catalysts under Industrially Relevant Conditions.

In preparation.

van Deelen, T. W., Yoshida, H., Oord, R., Zečević, J., Weckhuysen, B. M. \& de Jong, K. P. Cobalt nanocrystals on carbon nanotubes in the Fischer-Tropsch synthesis: the impact of support oxidation.

Appl. Catal. A Gen., DOI: 10.1016/j.apcata.2020.117441

van Deelen, T. W., Hernández Mejía, C. \& de Jong, K. P. Control of metal-support interactions in heterogeneous catalysts to enhance activity and selectivity.

Nat. Catal. 2, 955-970 (2019)

Hernández Mejía, C., van Deelen, T. W. \& de Jong, K. P. Activity enhancement of cobalt catalysts by tuning metal-support interactions.

Nat. Commun. 9, 4459 (2018).

van Deelen, T. W., Nijhuis, J. J., Krans, N. A., Zečević, J. \& de Jong, K. P. Preparation of Cobalt Nanocrystals Supported on Metal Oxides To Study Particle Growth in Fischer-Tropsch Catalysts.

ACS Catal. 8, 10581-10589 (2018).

van Deelen, T. W., Su, H., Sommerdijk, N. A. J. M. \& de Jong, K. P. Assembly and activation of supported cobalt nanocrystal catalysts for the Fischer-Tropsch synthesis.

Chem. Commun. 54, 2530-2533 (2018).

Ismail, A. S. M., Casavola, M., Liu, B., Gloter, A., van Deelen, T. W., Versluijs, M., Meeldijk, J. D., Stéphan, O., de Jong, K. P. \& de Groot, F. M. F. Atomic-Scale Investigation of the Structural and Electronic Properties of Cobalt-Iron Bimetallic Fischer-Tropsch Catalysts.

ACS Catal. 9, 7998-8011 (2019).

Xie, J., Paalanen, P. P., van Deelen, T. W., Weckhuysen, B. M., Louwerse, M. J. \& de Jong, K. P. Promoted cobalt metal catalysts suitable for the production of lower olefins from natural gas.

Nat. Commun. 10, 167 (2019). 
Sun, X., Suarez, A. I. O., Meijerink, M., Van Deelen, T., Ould-Chikh, S., Zečević, J., De Jong, K. P., Kapteijn, F. \& Gascon, J. Manufacture of highly loaded silica-supported cobalt Fischer-Tropsch catalysts from a metal organic framework.

Nat. Commun. 8, (2017).

Oschatz, M., Hofmann, J. P., van Deelen, T. W., Lamme, W. S., Krans, N. A., Hensen, E. J. M. \& de Jong, K. P. Effects of the Functionalization of the Ordered Mesoporous Carbon Support Surface on Iron Catalysts for the Fischer-Tropsch Synthesis of Lower Olefins.

ChemCatChem 9, 620-628 (2017).

Oschatz, M., Van Deelen, T. W., Weber, J. L., Lamme, W. S., Wang, G., Goderis, B., Verkinderen, O., Dugulan, A. I. \& De Jong, K. P. Effects of calcination and activation conditions on ordered mesoporous carbon supported iron catalysts for production of lower olefins from synthesis gas.

Catal. Sci. Technol. 6, (2016).

van Haasterecht, T., van Deelen, T. W., Ludding, C. C. L., de Jong, K. P. \& Bitter, J. H. Transformations of polyols to organic acids and hydrogen in aqueous alkaline media.

Catal. Sci. Technol. 4, 2353-2366 (2014). 


\section{Presentations}

\section{Oral presentations}

van Deelen, T. W. \& de Jong, K. P. Cobalt Nanocrystals as Model Catalysts for the FischerTropsch Synthesis. $12^{\text {th }}$ Natural Gas Conversion Symposium, San Antonio, TX, US (June 2019)

van Deelen, T. W. \& de Jong, K. P. Assembly and Activation of Cobalt Nanocrystal Catalysts for the Fischer-Tropsch Synthesis.

$13^{\text {th }}$ European Congress on Catalysis, Florence, Italy (August 2017)

van Deelen, T. W. \& de Jong, K. P. Assembly and Activation of Cobalt Nanocrystal Catalysts for the Fischer-Tropsch Synthesis.

$25^{\text {th }}$ North American Catalysis Society Meeting, Denver, CO, US (June 2017)

van Deelen, T. W. \& de Jong, K. P. Assembly and Activation of Cobalt Nanocrystal Catalysts for the Fischer-Tropsch Synthesis.

$18^{\text {th }}$ Netherlands Catalysis and Chemistry Conference (NCCC), Noordwijkerhout, The Netherlands (April 2017)

\section{Poster presentations}

van Deelen, T. W., Nijhuis, J. J., Krans, N. A., Zečević, J. \& de Jong, K. P. SupportDependent Growth of Cobalt Nanocrystals Applied as Fischer-Tropsch Catalysts

$19^{\text {th }}$ Netherlands Catalysis and Chemistry Conference (NCCC), Noordwijkerhout, The Netherlands (April 2018)

van Deelen, T. W., Su, H., Sommerdijk, N. A. J. M. \& de Jong, K. P. Supported Cobalt Colloids as Model Catalysts in the Fischer-Tropsch Synthesis

$17^{\text {th }}$ Netherlands Catalysis and Chemistry Conference (NCCC), Noordwijkerhout, The Netherlands (April 2016) 


\section{Acknowledgements}

This thesis marks the end of a period of 7 years in the Inorganic Chemistry and Catalysis group that started as a BSc student. It has been a truly special and memorable time, for which I'm grateful to several people.

Krijn, ik wil jou hartelijk bedanken voor de kans die je me hebt gegeven om mijn promotieonderzoek onder jouw begeleiding uit te voeren. Gedurende de jaren heb jij mij uitgedaagd en zoveel vrijheid gegeven als ik zelf wilde, maar tegelijkertijd kon ik altijd even langslopen om te overleggen en hield je toch de voortgang in de gaten. Hoe goed ik ook dacht voorbereid te zijn, jij wist altijd met een nieuwe vraag of invalshoek te komen, waardoor projecten de goede kant op gestuurd werden. Zeker de internationale congressen deden me beseffen dat je een wereldwijd erkende expert bent in het vakgebied en ik beschouw het dan ook als een voorrecht om een flinke tijd met je te hebben samengewerkt.

Graag wil ik ook de sponsors van mijn project, NWO en Shell Global Solutions, bedanken. In het bijzonder wil ik Peter Munnik, Johan den Breejen, Leendert Bezemer, Sander van Bavel en Heiko Oosterbeek bedanken voor de begeleiding vanuit Shell en de discussies, waar vaak weer nieuwe inzichten en ideeën uit voortkwamen. Deze bijeenkomsten hebben het onderzoek mede gevormd en heb ik altijd zeer gewaardeerd. Ook wil ik Avantium bedanken voor de prettige samenwerking m.b.t. de Flowrence.

I am also grateful for the meetings and collaborations within our CHIPP project. Hao Su, Heiner Friedrich and Nico Sommerdijk, thank you for your input, especially on the possibilities of (cryo-)TEM. Hao, thank you for performing the cryo-TEM experiments, which were certainly my most logistically challenging experiments, with NC synthesis in Utrecht in one building, vitrification in another and analysis in Eindhoven all within a few hours. I would also like to thank the CHIPP-colleagues at Utrecht, Thomas Hartman, Ilse van Ravenhorst, José Moya-Cancino and Bert Weckhuysen, for the discussions and collaborations.

Ik heb tijdens mijn PhD meerdere studenten mogen begeleiden. Iris en Jelle, het was ontzettend mooi om te zien hoe jullie je MSc-onderzoeksproject eigen maakten en uiteindelijk zelf vormgaven als grotendeels zelfstandige onderzoekers. Koen, jouw literatuurstudie is erg nuttig geweest om een overzicht te krijgen van het veld en Lauren, ik heb ervan genoten om tijdens jouw BSc-onderzoeksproject samen een zijweg te onderzoeken en uit te vinden wat de beste aanpak was. Voor alle studenten geldt dat ik jullie met veel plezier heb begeleid en ik wens jullie heel veel succes toe.

$\mathrm{Al}$ dit werk zou zeker niet mogelijk zijn geweest zonder de technische staf: $\mathrm{Ad}^{\mathrm{M}}, \mathrm{Ad}^{\mathrm{E}}$, Hans, Marjan, Rien, Fouad, Jan Willem, Pascal, Oscar, Herrick, Dennie en Ramon, jullie zijn onmisbaar om het lab draaiend te houden. Dank voor alle hulp en tijd om even een 'korte' vraag te beantwoorden. Net zo onmisbaar, maar dan voor de organisatie van de groep: het secretariaat. Dymph, Ilonka, Monique, Iris en anderen, ik wil jullie ook hartelijk bedanken voor de ondersteuning.

I will not attempt to list all the individual colleagues who contributed in some way to my project, because there are simply too many. Rather, I would like to thank the whole ICC 
group for their help, the open atmosphere and the fun moments and I would like to mention a few people in particular.

De attente collega's, Wouter, Thomas, Joris, Carolien, Marjolein, Anne-Eva en Robin, jullie advies, hulp en relativeringsvermogen bij problemen waren onmisbaar, evenals de regelmatige updates over wat er op intranet stond. Ook op het moment dat de boog even niet gespannen stond, werd jullie assistentie in de vorm van lunchloopjes, pizzaburgers, broccoli bij het dorp en andere gekkigheid zeer gewaardeerd.

Carlos, your creativity, critical view, and love for Japanese color combinations made it great fun to work with you as \#contributedequally author. I also enjoyed attending all international conferences with you. There are few people with whom I travelled so many kilometers. Have a good one!

Lennart, my first official buddy in the group, thanks for the (technical) support with the Flowrence, for your wild ideas for possible experiments and for lending me your car in the name of science. I'm glad that we managed to complete the FT corner in the end.

Lisette, wij waren een paar jaar bureauburen en naast dat dat heel gezellig was, was het ook praktisch, want jij leek soms het orakel van de groep. Iedereen kwam met zijn vragen naar jou, en dan wist je ook het antwoord nog!

Arjan, ik heb veel van je geleerd tijdens mijn MSc-onderzoek, maar zeker ook in het begin van mijn PhD bij het opstarten in mijn rol als beheerder van de Flowrence. Bedankt dat je altijd tijd voor me maakte.

Ramon, dikke lekker dat we op het eind toch nog de in situ XRD experimenten konden doen! Niks is jou teveel moeite en dat maakt het heel fijn samenwerken.

Hideto, you are a true expert in electron microscopy and I am very happy that you were willing to perform the tomography experiments.

The TEM team consisting of Hans, Jovana, Wouter, Nynke, Mark, Lars, and others, thank you for the numerous hours that you spent in the dark (and later comfortably in the light) analyzing my samples. The TEM results you obtained have been invaluable for my research project.

JX, Martin, Nynke, Jogchum, Roxana, Justine, Kang, Rolf and Remco, I enjoyed working with you as part of the Flowrence (II) team, trying to figure out how to perform experiments, optimize the setup, or solve whatever problem we encountered. Thanks for the collaboration on several projects!

Tot slot wil ik mijn familie bedanken voor hun onvoorwaardelijke steun. Hemmy, Marjolijn, Joost, Emma, Judith, bedankt dat ik altijd met mijn verhalen bij jullie terecht kon, ook al was waarschijnlijk niet altijd duidelijk waar ik nou eigenlijk mee bezig was. Dit heeft mij in ieder geval heel erg geholpen. Marlijn, jij hebt dit hele traject vanaf de eerste rij gevolgd en jij bent een enorme steun geweest. Jij hielp mij tegenslagen te relativeren, jouw drive, energie en creatieve ideeën zijn inspirerend, en bovenal is het met jou nooit saai. Bedankt voor alles. 


\section{Curriculum vitae}

Tom van Deelen was born on December 9, 1991 in Soest, The Netherlands. After completing his pre-university education at the Johan van Oldenbarnevelt Gymnasium in Amersfoort in 2009, he studied chemistry at Utrecht University and obtained a bachelor degree in 2012. He continued his education at Utrecht University and graduated from the master program 'Nanomaterials: Chemistry and Physics' in 2014. His main research project was entitled 'Niobia-Promoted Co/CNT Catalysts for the Fischer-Tropsch Synthesis' and was supervised by dr.

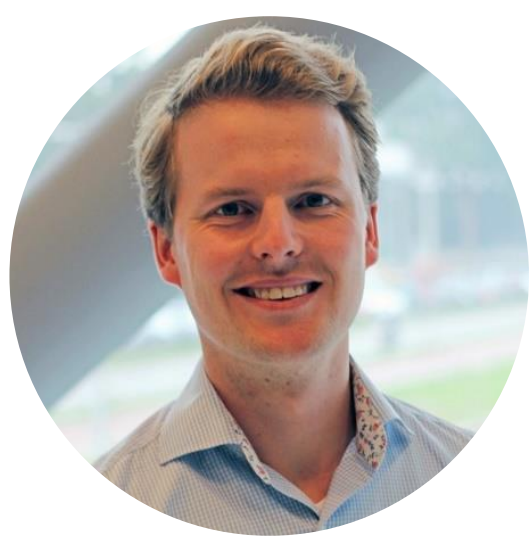
Arjan den Otter and prof. Krijn de Jong. In addition, he worked on the synthesis of novel hydroprocessing catalysts during an industrial internship at Albemarle in Amsterdam under supervision of dr. Jana Juan-Alcañiz and dr. Jaap Bergwerff

In 2014, Tom started a PhD research project on the application of colloidal cobalt nanocrystals in the synthesis of supported cobalt catalysts for the Fischer-Tropsch synthesis in the Inorganic Chemistry and Catalysis group at Utrecht University under supervision of prof. Krijn de Jong. The results of this research are described in this thesis. 